\title{
Phenoxyamidine $\mathrm{Zn}$ and Al complexes: synthesis, characterization and use in the ROP of Lactide
}

Florian Chotard,$^{\dagger}$ Rosita Lapenta, ${ }^{\S, \#}$ Anaëlle Bolley, ${ }^{\S}$ Audrey Trommenschlager, ${ }^{\dagger}$ Cédric Balan,${ }^{\dagger}$ Jérôme Bayardon, ${ }^{\dagger}$ Raluca Malacea-Kabbara,${ }^{\dagger}$ Quentin Bonnin,${ }^{\dagger}$ Ewen Bodio, ${ }^{\dagger}$ Hélène Cattey, ${ }^{\dagger}$ Philippe Richard,${ }^{\dagger}$ Stefano Milione, ${ }^{*}$ Alfonso Grassi, ${ }^{\#}$ Samuel Dagorne ${ }^{*}{ }^{\S}$ and Pierre Le Gendre* ${ }^{\dagger}$

†Institut de Chimie Moléculaire de l’Université de Bourgogne (ICMUB, UMR-CNRS 6302), Université de Bourgogne Franche-Comté

§Institut de Chimie de Strasbourg (UMR-CNRS 7177), Université de Strasbourg

\# Dipartimento di Chimica e Biologia, Università degli Studi di Salerno, via Giovanni Paolo II, 132-84084

Fisciano (SA), Italy

\section{Table of contents}

${ }^{1} \mathrm{H},{ }^{13} \mathrm{C}\left\{{ }^{1} \mathrm{H}\right\}$, COSY, HSQC, HMBC, VT ${ }^{1} \mathrm{H}$ NMR, ${ }^{1} \mathrm{H}^{15} \mathrm{~N}$ HMBC, ATR-FT-IR spectra of L1H S2

${ }^{1} \mathrm{H}$ NMR monitoring of the evolution of $\mathbf{L 1 H}$ into 2-Phenyl-4H-1,3-benzoxazine. S6

${ }^{1} \mathrm{H},{ }^{13} \mathrm{C}\left\{{ }^{1} \mathrm{H}\right\}$ spectra of 2-Phenyl-4H-1,3-benzoxazine $\quad$ S8

${ }^{1} \mathrm{H},{ }^{13} \mathrm{C}\left\{{ }^{1} \mathrm{H}\right\}$, COSY, HSQC, HMBC spectra of L1Na $\quad$ S6

${ }^{1} \mathrm{H},{ }^{13} \mathrm{C}\left\{{ }^{1} \mathrm{H}\right\}$, COSY, HSQC, HMBC, ${ }^{1} \mathrm{H}^{15} \mathrm{~N}$ HMBC, ATR-FT-IR spectra of [L1 $\left.\mathbf{H}_{2}\right][\mathbf{B r}] \quad \mathrm{S} 10$

${ }^{1} \mathrm{H},{ }^{13} \mathrm{C}\left\{{ }^{1} \mathrm{H}\right\}$, COSY, NOESY, HSQC, HMBC, VT ${ }^{1} \mathrm{H}$ NMR, ${ }^{1} \mathrm{H}{ }^{15} \mathrm{~N}$ HMBC, ATR-FT-IR spectra and crystal $\mathrm{S} 15$ structure and table of crystal data of $\mathbf{L 2 H}$

${ }^{1} \mathrm{H},{ }^{13} \mathrm{C}\left\{{ }^{1} \mathrm{H}\right\}$, HSQC, ${ }^{1} \mathrm{H}^{15} \mathrm{~N}$ HMBC, ATR-FT-IR spectra of of $\mathbf{L 3 H}$ and $\mathbf{L} 4 \mathbf{H} \quad$ S21

${ }^{1} \mathrm{H},{ }^{13} \mathrm{C}\left\{{ }^{1} \mathrm{H}\right\}, \mathrm{COSY}, \mathrm{HSQC}, \mathrm{HMBC}$, DOSY, crystal structure and table of crystal data of 1a 26

${ }^{1} \mathrm{H},{ }^{13} \mathrm{C}\left\{{ }^{1} \mathrm{H}\right\}$, COSY, HSQC, HMBC, crystal structure and table of crystal data of 2a

${ }^{1} \mathrm{H},{ }^{13} \mathrm{C}\left\{{ }^{1} \mathrm{H}\right\}$, COSY, HSQC, HMBC of $\mathbf{3 a}$ and $\mathbf{4 a} \quad S 35$

${ }^{1} \mathrm{H},{ }^{13} \mathrm{C}\left\{{ }^{1} \mathrm{H}\right\}$, HSQC, HMBC, crystal structure and table of crystal data of 1a' $\quad \mathrm{S} 40$

${ }^{1} \mathrm{H},{ }^{13} \mathrm{C}\left\{{ }^{1} \mathrm{H}\right\}, \mathrm{COSY}, \mathrm{HSQC}, \mathrm{HMBC}$, crystal structure and table of crystal data of $\mathbf{1 b} \quad \mathrm{S} 43$

${ }^{1} \mathrm{H},{ }^{13} \mathrm{C}\left\{{ }^{1} \mathrm{H}\right\}$, HSQC, HMBC, VT ${ }^{1} \mathrm{H}$ NMR, crystal structure and table of crystal data of $\mathbf{2 b} \quad \mathrm{S} 47$

${ }^{1} \mathrm{H},{ }^{13} \mathrm{C}\left\{{ }^{1} \mathrm{H}\right\}$, COSY, HSQC, HMBC of $\mathbf{3 b}$ and $\mathbf{4 b} \quad$ S51

${ }^{1} \mathrm{H}$ NMR monitoring of 1a alcoholysis; ${ }^{1} \mathrm{H}$ NMR spectrum, crystal structure and table of crystal data of 1a" S56

Graph of the PLA $M_{\mathrm{n}}$ values as a function of lactide conversion, Graph of $\ln ([\mathrm{M}] 0 /[\mathrm{M}])$ versus time, GPC S58 analysis, MALDI-TOF spectra 


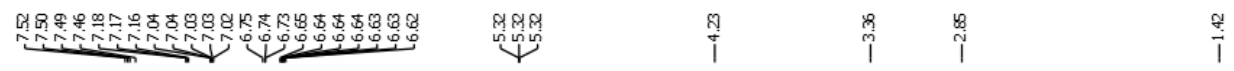<smiles>CN(Cc1ccccc1O)C(=NCc1ccccc1)c1ccccc1</smiles>

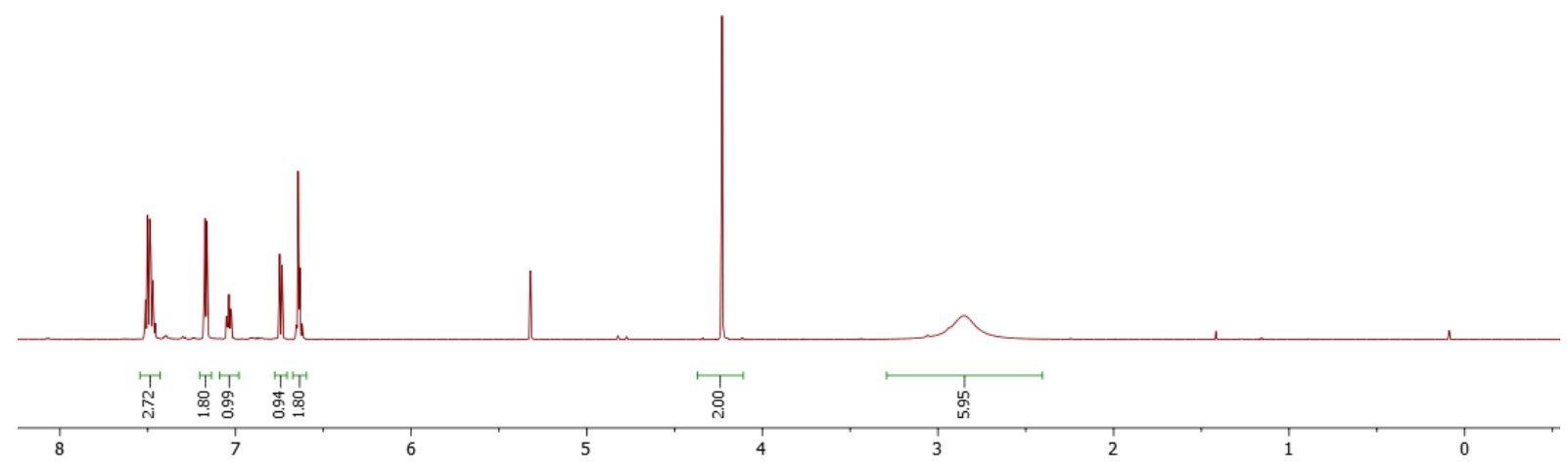

Figure S1: ${ }^{1} \mathrm{H}$ NMR (600 MHz, $\left.\mathrm{CD}_{2} \mathrm{Cl}_{2}\right)$ of $(E)-N$ '-(2-hydroxybenzyl)- $N, N$-dimethylbenzamidine $\mathbf{L 1 H}$

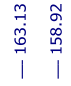<smiles>CN(C)C(=NCc1ccccc1O)c1ccccc1</smiles>

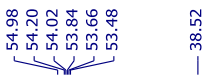

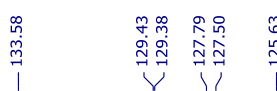
$\stackrel{1}{1}$

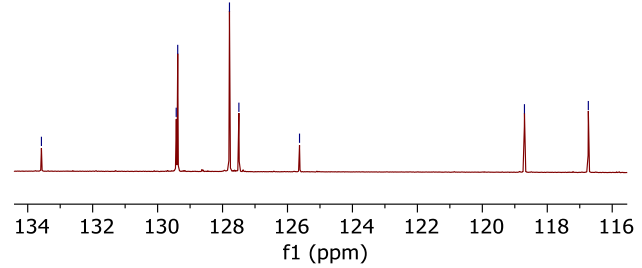
f1 (ppm)
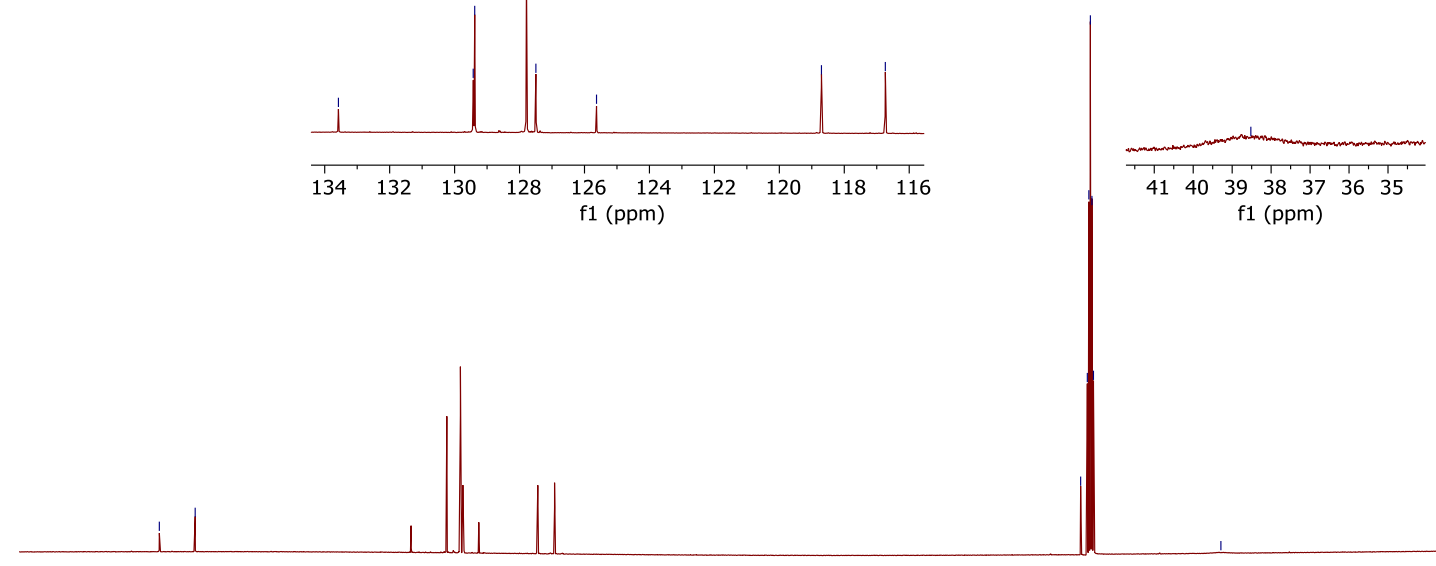

$90 \quad 80$

Figure S2: ${ }^{13} \mathrm{C}\left\{{ }^{1} \mathrm{H}\right\}$ NMR $\left(151 \mathrm{MHz}, \mathrm{CD}_{2} \mathrm{Cl}_{2}\right)$ of $(E)-N$ '-(2-hydroxybenzyl)- $N, N$-dimethylbenzamidine $\mathbf{L 1 H}$ 


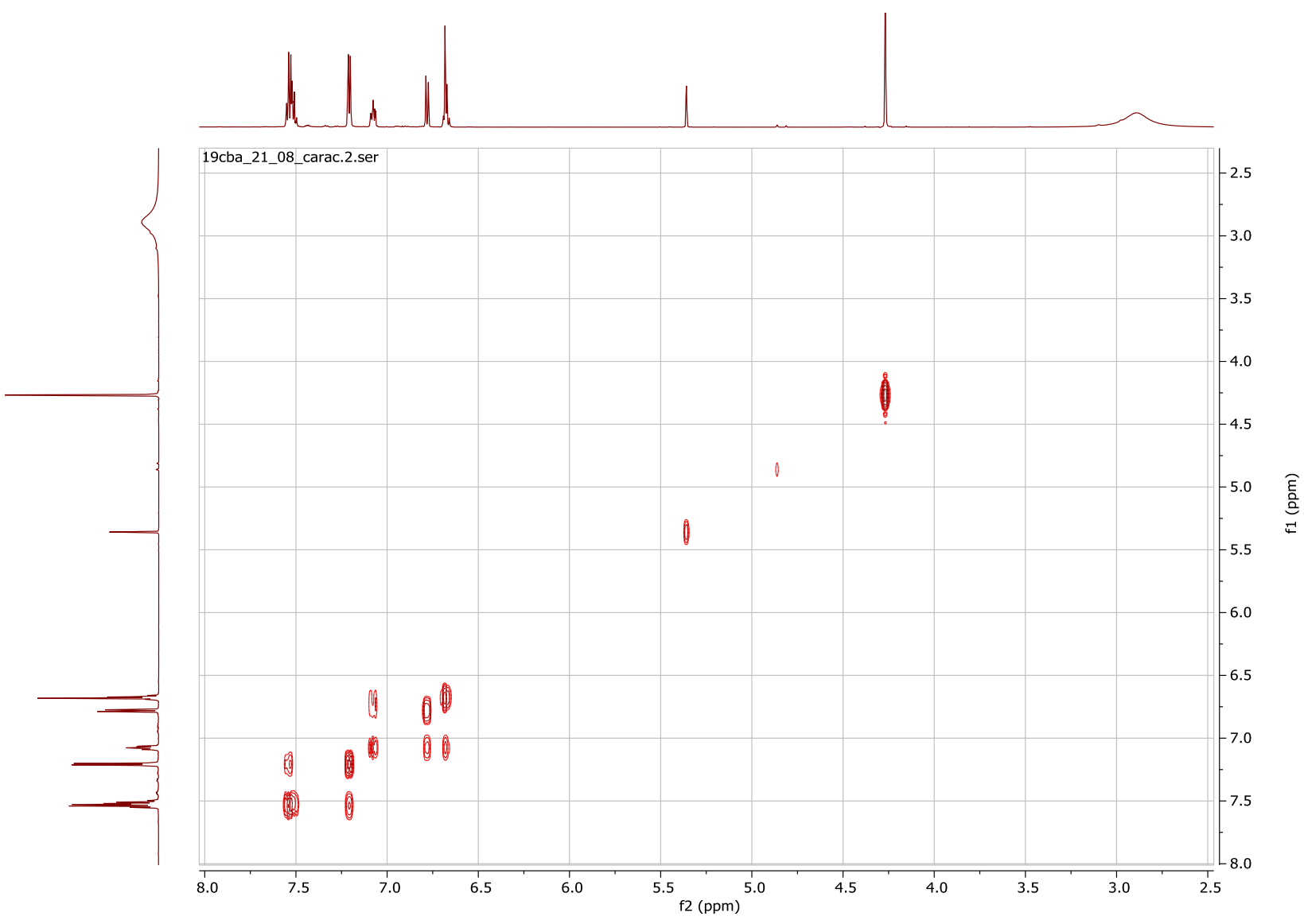

Figure S3: ${ }^{1} \mathrm{H}{ }^{1} \mathrm{H} \operatorname{COSY}\left(600 \mathrm{MHz}, \mathrm{CD}_{2} \mathrm{Cl}_{2}\right)$ of $(E)-N$ '-(2-hydroxybenzyl)- $N, N$-dimethylbenzamidine $\mathbf{L 1 H}$

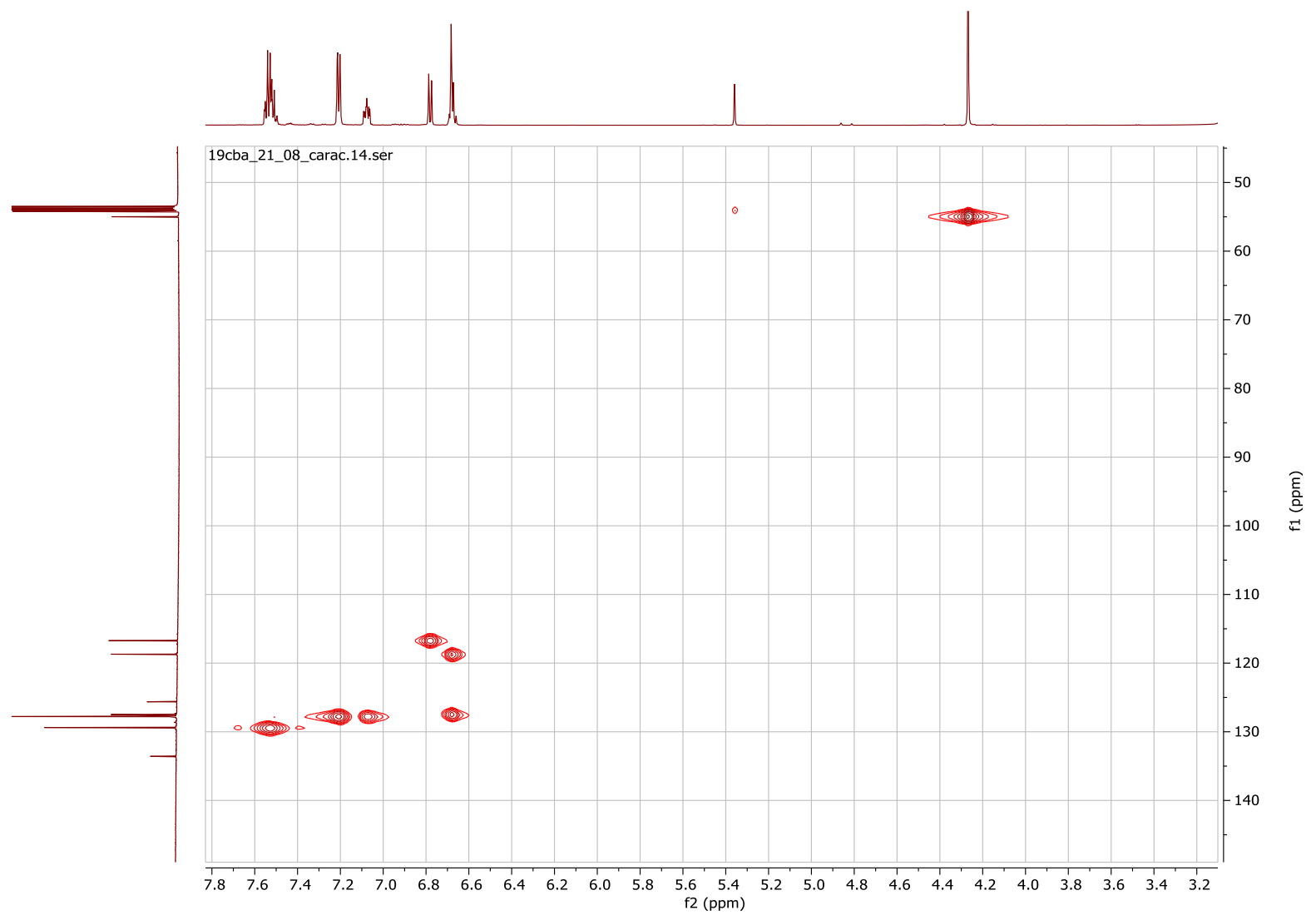

Figure S4: ${ }^{1} \mathrm{H}{ }^{13} \mathrm{C}$ HSQC $\left(600 \mathrm{MHz} / 151 \mathrm{MHz}, \mathrm{CD}_{2} \mathrm{Cl}_{2}\right)$ of $(E)-N$ '-(2-hydroxybenzyl)- $N, N$-dimethylbenzamidine $\mathbf{L 1 H}$ 


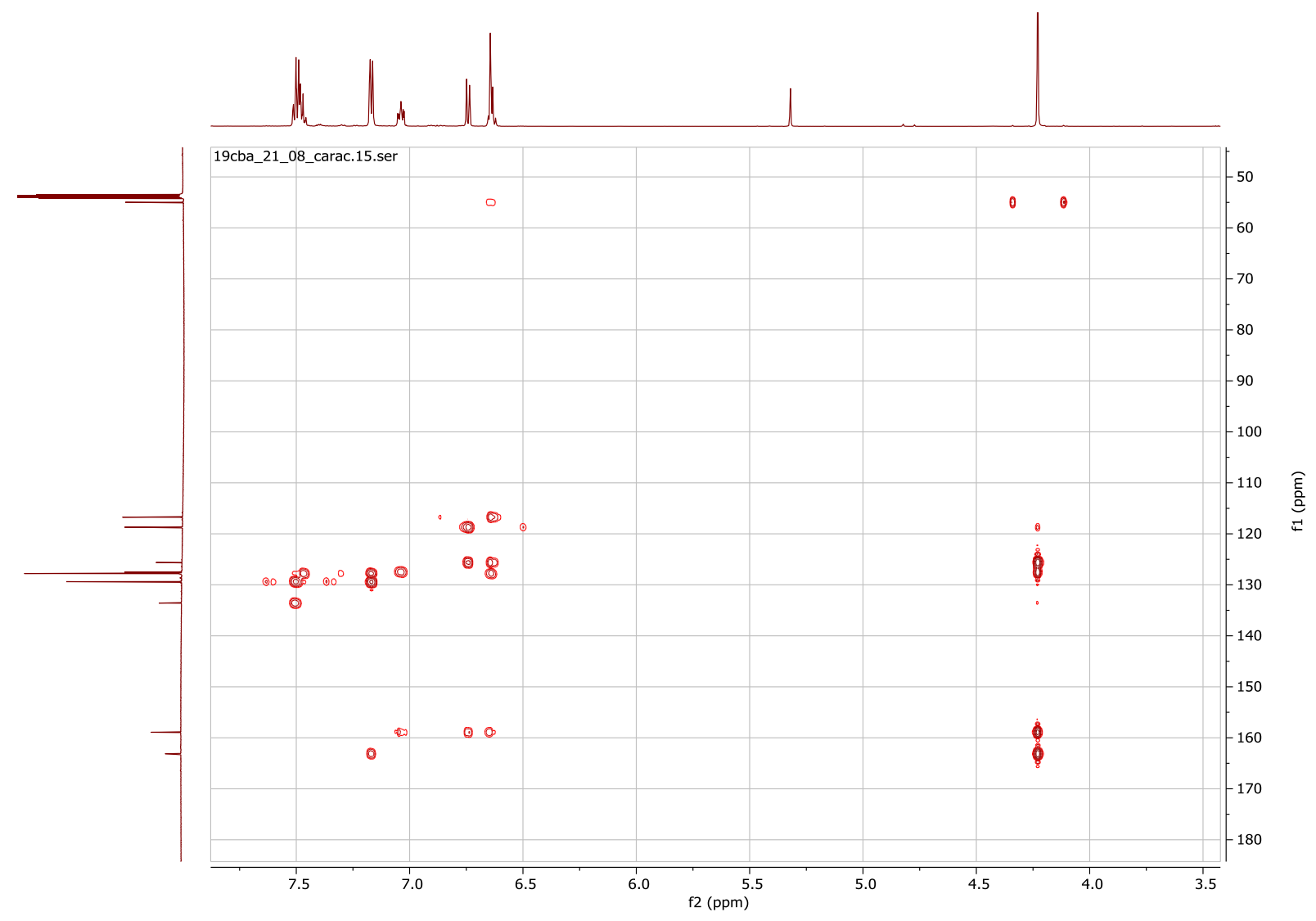

Figure S5: ${ }^{1} \mathrm{H}^{13} \mathrm{C} \mathrm{HMBC}\left(600 \mathrm{MHz} / 151 \mathrm{MHz}, \mathrm{CD}_{2} \mathrm{Cl}_{2}\right)$ of $(E)-N$ '-(2-hydroxybenzyl)- $N, N$-dimethylbenzamidine $\mathbf{L 1 H}$

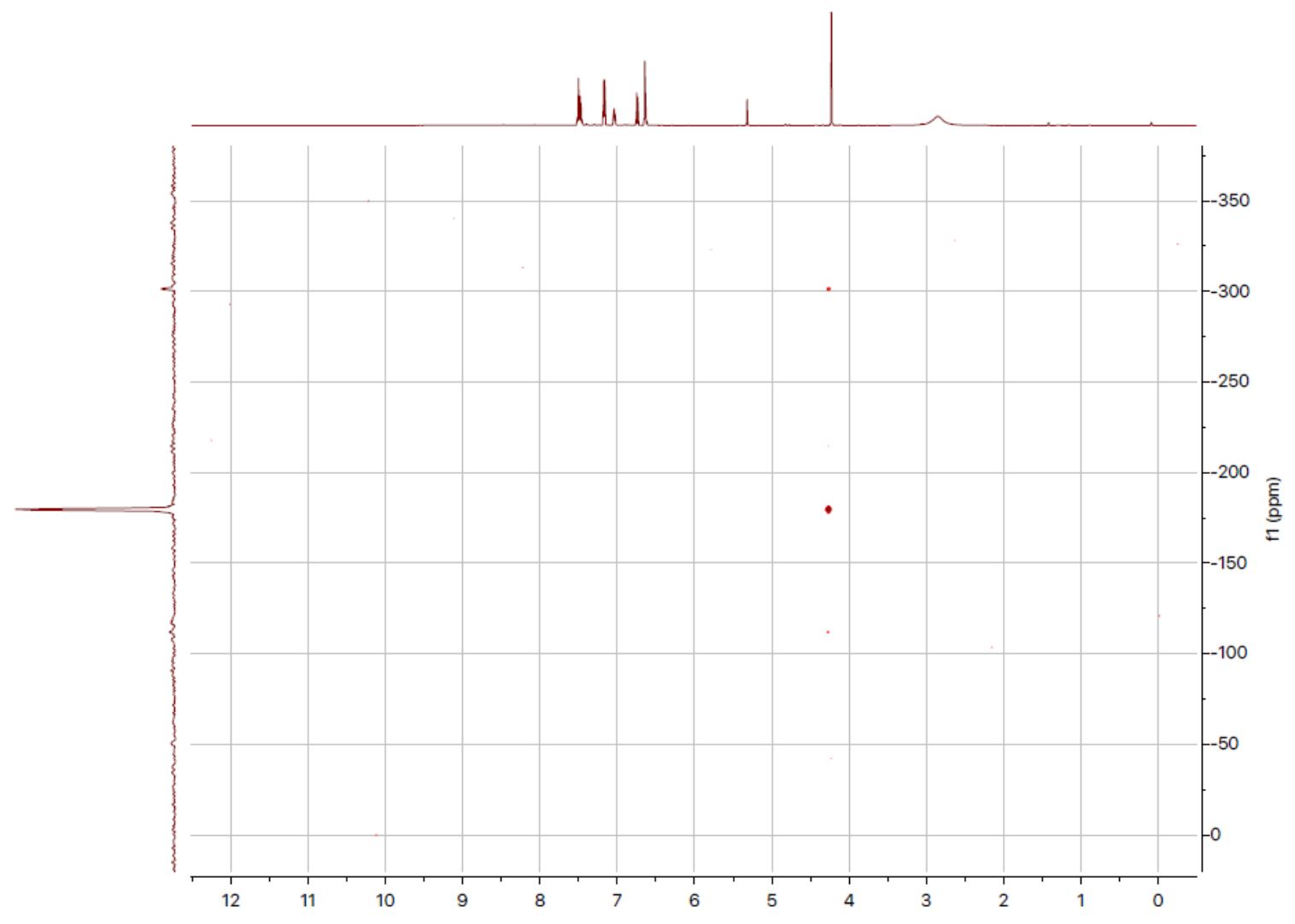

Figure S6: ${ }^{1} \mathrm{H}^{15} \mathrm{~N} \mathrm{HMBC}\left(600.23 \mathrm{MHz} / 43.3 \mathrm{MHz}, \mathrm{CD}_{2} \mathrm{Cl}_{2}\right.$,) of $(E)-N$ '-(2-hydroxybenzyl)- $N, N$-dimethylbenzamidine $\mathbf{L 1 H}$ 


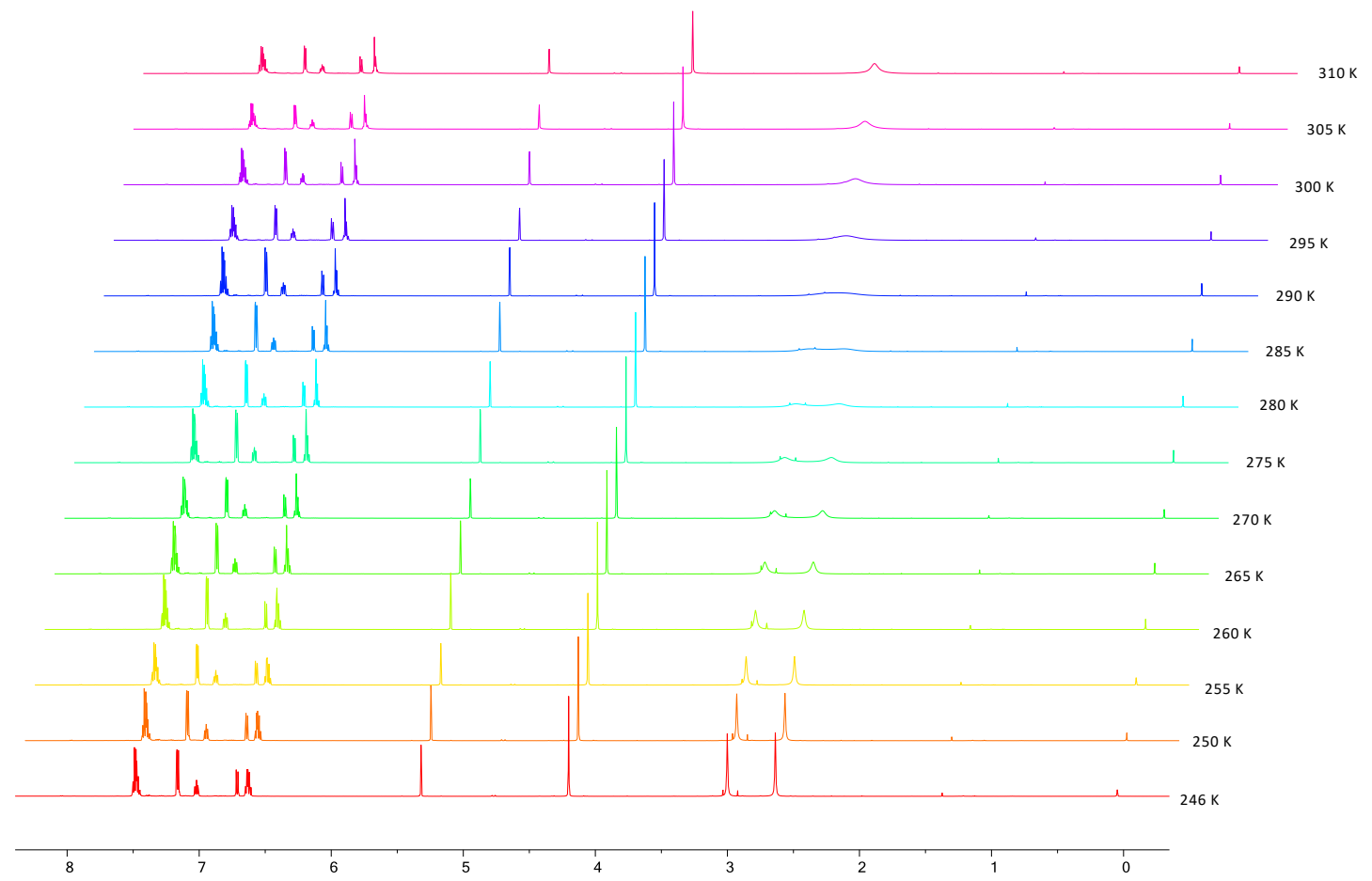

Figure S7: Variable Temperature ${ }^{1} \mathrm{H}$ NMR $\left(600 \mathrm{MHz}, \mathrm{CD}_{2} \mathrm{Cl}_{2}\right)$ of $\mathbf{L 1 H}$

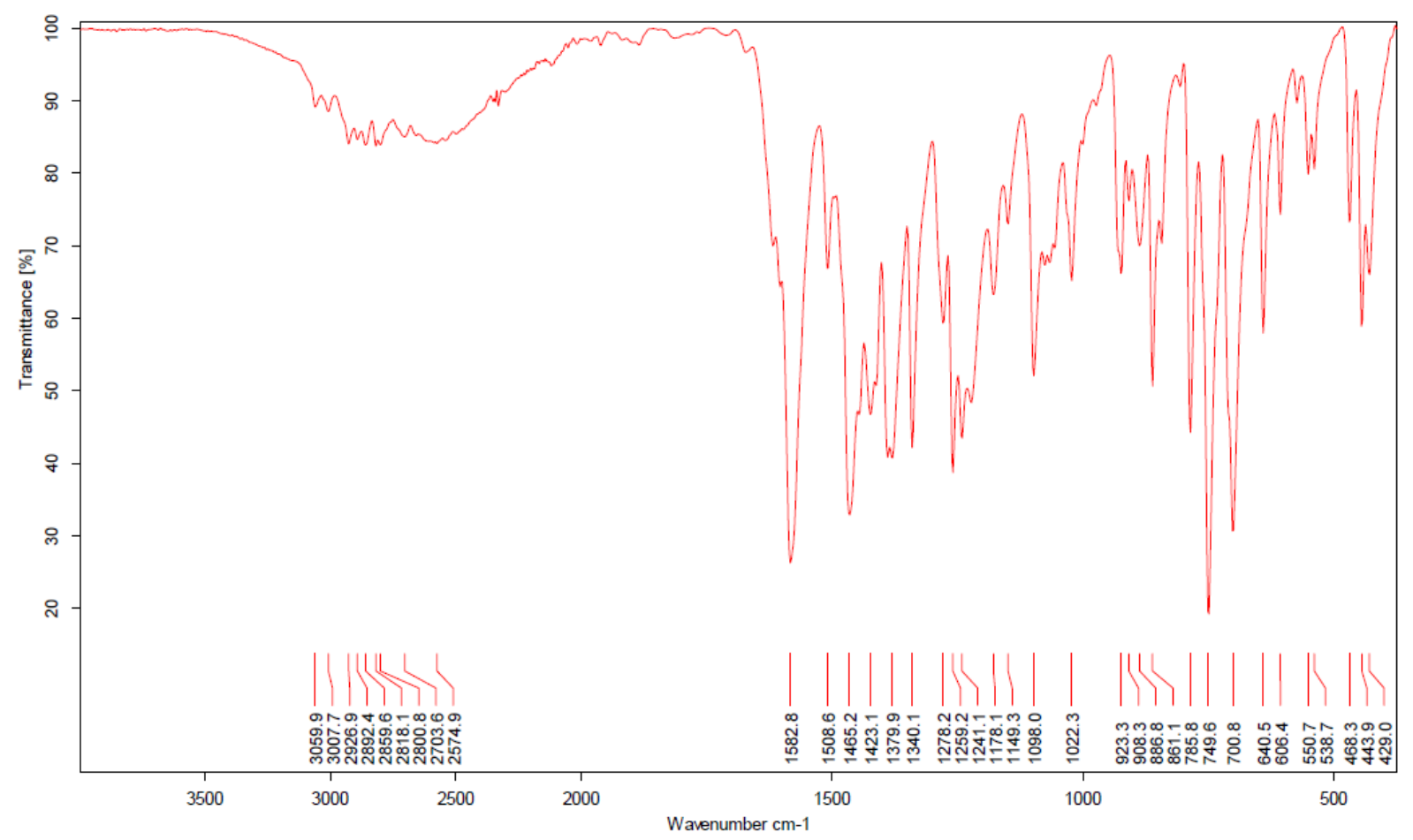

Figure S8: ATR-FT-IR spectrum of (E)- $N$ '-(2-hydroxybenzyl)- $N, N$-dimethylbenzamidine L1H 


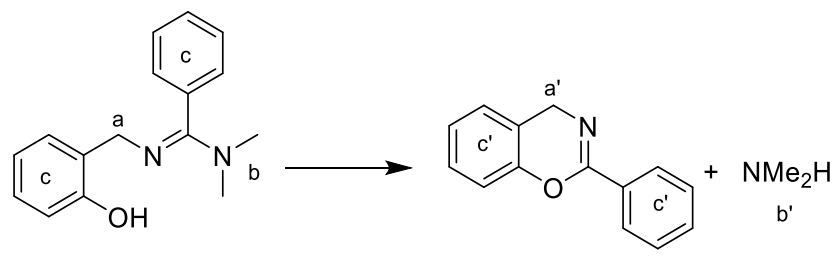

L1H

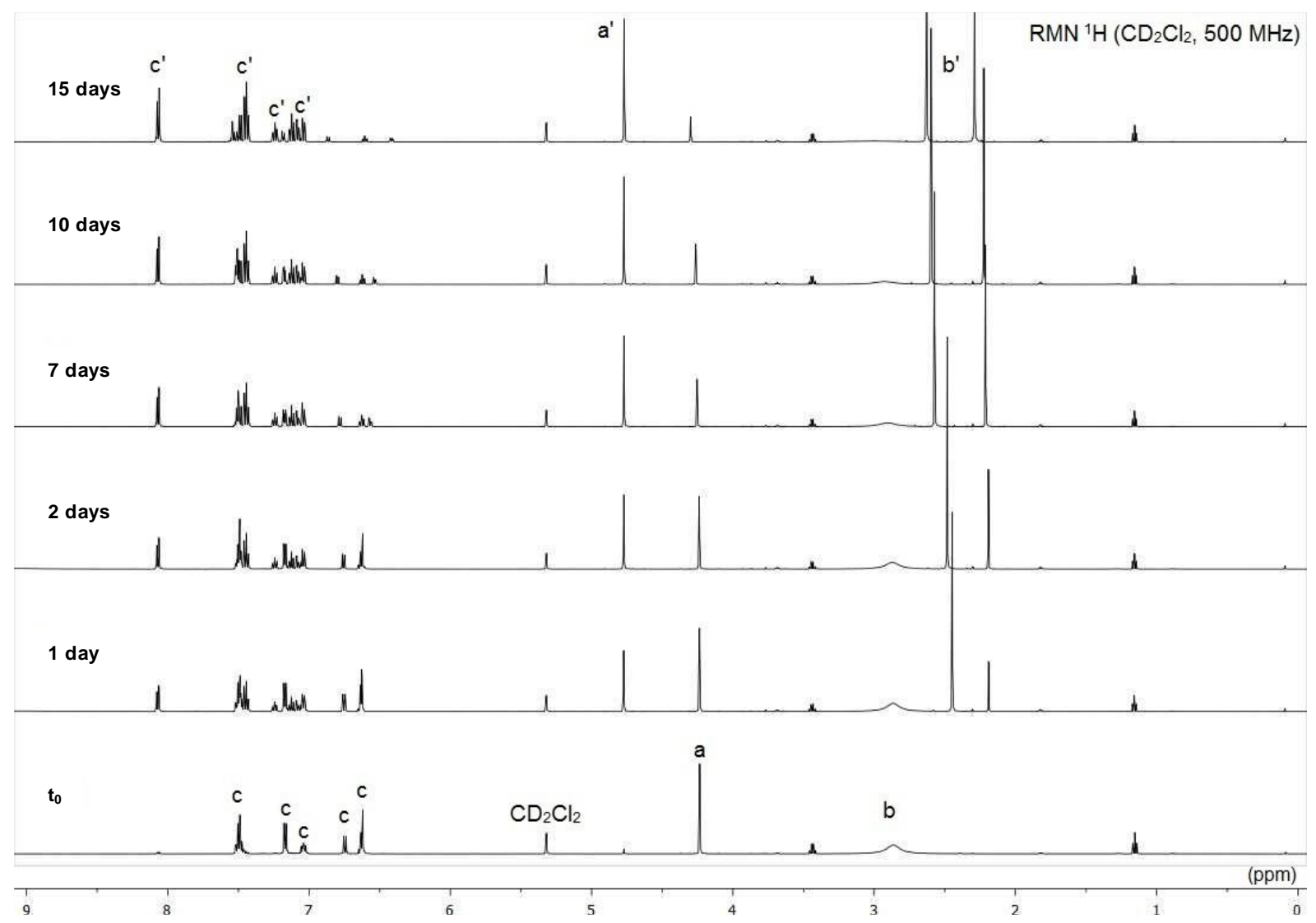

Figure S9: ${ }^{1} \mathrm{H}$ NMR spectra $(500 \mathrm{MHz}, 298 \mathrm{~K})$ recorded after different time intervals of a $\mathrm{CD}_{2} \mathrm{Cl}_{2}$ solution of $\mathbf{L 1 H}$ at room temperature showing the evolution of $\mathbf{L 1 H}$ into 2-Phenyl-4H-1,3-benzoxazine.

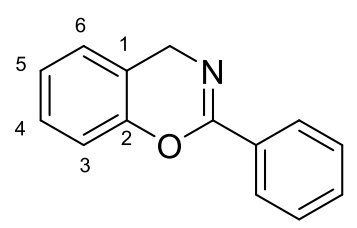

2-Phenyl-4H-1,3-benzoxazine was obtained as a by-product of the reaction of deprotonation of (E)- $N$ '-(2-hydroxybenzyl)- $N, N$-dimethylbenzamidinium bromide L1H2 with $\mathrm{NaH}$. It was purified by silica column chromatography using petroleum ether/ethyl acetate system as eluent and isolated as a white solid (370 mg). HRMS (ESI-pos): calcd for $\left[\mathrm{C}_{14} \mathrm{H}_{12} \mathrm{NO}\right]^{+}$ $[\mathrm{M}+\mathrm{H}]^{+}$: 210.09134. Found: $210.09040(-4.5 \mathrm{ppm})$. Elemental Analysis: calcd for $\mathrm{C}_{14} \mathrm{H}_{11} \mathrm{NO}$ : C, 80.36; H, 5.30, N 6.69. Found: C, 79.89; H, 5.30; N, 6.92. ${ }^{1} \mathrm{H}$ NMR $(300$ $\left.\mathrm{MHz}, \mathrm{CDCl}_{3}\right): \delta(\mathrm{ppm})=8.11-8.04(\mathrm{~m}, 2 \mathrm{H}, o-\mathrm{Ph}), 7.53-7.40(\mathrm{~m}, 2 \mathrm{H}+1 \mathrm{H}, m-\mathrm{Ph}$ and $p-\mathrm{Ph}), 7.24(\mathrm{~m}, 1 \mathrm{H}, \mathrm{Ar} \mathrm{H})_{4}, 7.12$ (pseudo td, ${ }^{3} J_{\mathrm{HH}}=7.3,{ }^{4} J_{\mathrm{HH}}=1.2 \mathrm{~Hz}, 1 \mathrm{H}, \mathrm{Ar} \mathrm{H}$ ), $7.07\left(\mathrm{~m}, 1 \mathrm{H}, \mathrm{Ar} \mathrm{H}_{6}\right.$ overlapping with $\mathrm{Ar} \mathrm{H}_{3}$ and $\left.\mathrm{Ar} \mathrm{H} \mathrm{H}_{5}\right), 7.03\left(\mathrm{dd},{ }^{3} J_{\mathrm{HH}}\right.$ $=8.0 \mathrm{~Hz},{ }^{4} J_{\mathrm{HH}}=1.2 \mathrm{~Hz}, 1 \mathrm{H}, \mathrm{Ar} \mathrm{H} \mathrm{H}_{3}$ overlapping with $\left.\mathrm{Ar} \mathrm{H}_{6}\right) 4.80\left(\mathrm{~s}, 2 \mathrm{H}, \mathrm{CH}_{2}\right) .{ }^{13} \mathrm{C}\left\{{ }^{1} \mathrm{H}\right\} \mathrm{NMR}(75 \mathrm{MHz}, \mathrm{THF}-\mathrm{d} 8): \delta$ $(\mathrm{ppm})=152.9\left(\mathrm{C}_{\mathrm{q}}, \mathrm{C}=\mathrm{N}\right), 149.6\left(\mathrm{C}_{\mathrm{q}}, \mathrm{C}-\mathrm{O}\right), 132.4\left(\mathrm{C}_{\mathrm{q}}, i-\mathrm{Ph}\right), 131.1(\mathrm{CH}, p-\mathrm{Ph}), 128.4(\mathrm{CH}, m-\mathrm{Ph}), 128.2(\mathrm{CH}, \mathrm{Ar} \mathrm{C})$, $\left.127.4(\mathrm{CH}, o-\mathrm{Ph}), 126.1\left(\mathrm{CH}, \mathrm{Ar} \mathrm{C}_{6}\right), 124.9(\mathrm{CH}, \mathrm{Ar} \mathrm{C})_{5}\right), 119.5\left(\mathrm{Cq}, \mathrm{Ar} \mathrm{C}_{1}\right), 115.7\left(\mathrm{CH}, \mathrm{Ar} \mathrm{C}_{3}\right), 45.5\left(\mathrm{CH}_{2}\right)$. 


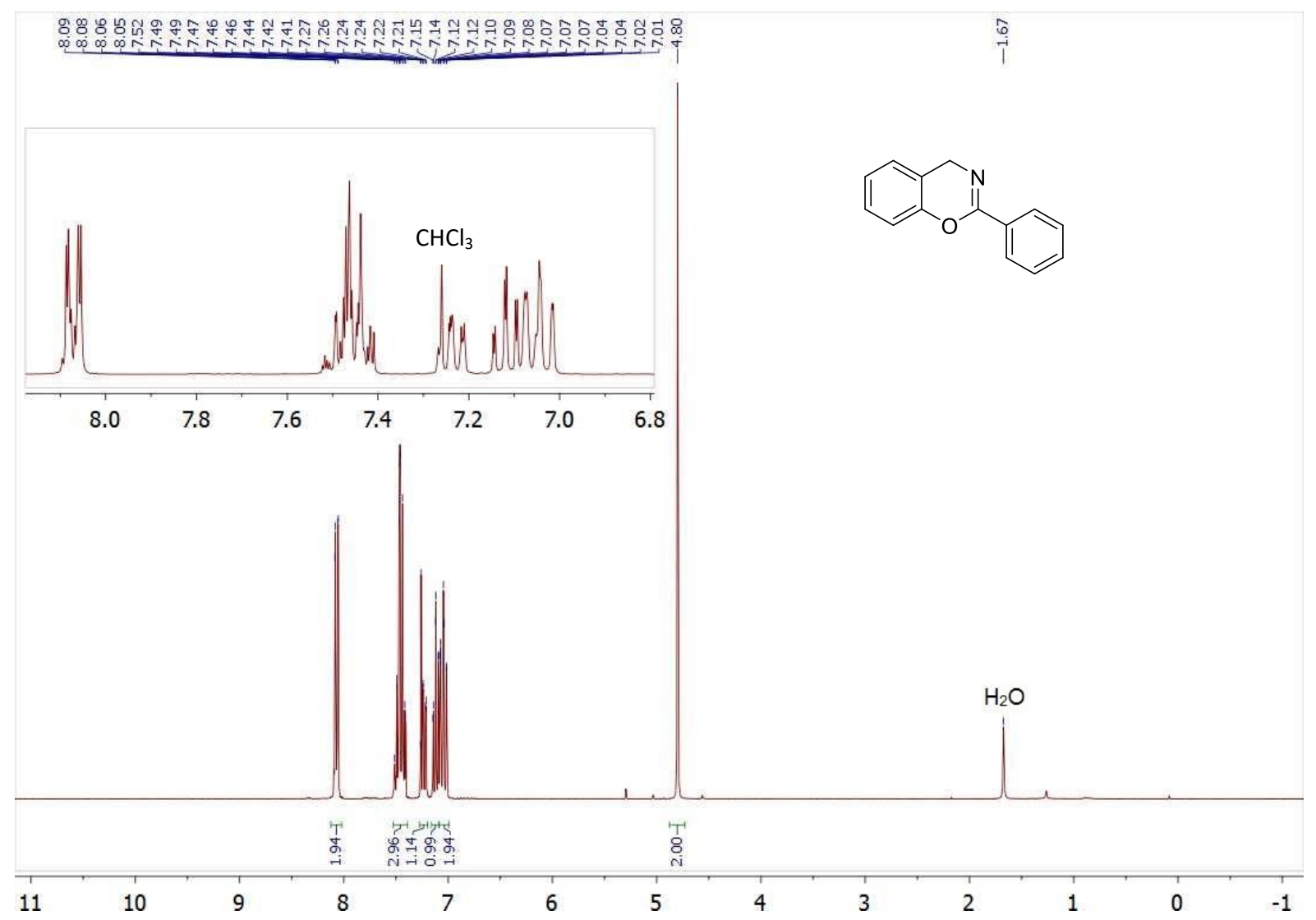

Figure S10: ${ }^{1} \mathrm{H}$ NMR (300 MHz, $\mathrm{CDCl}_{3}$ ) of 2-Phenyl-4H-1,3-benzoxazine

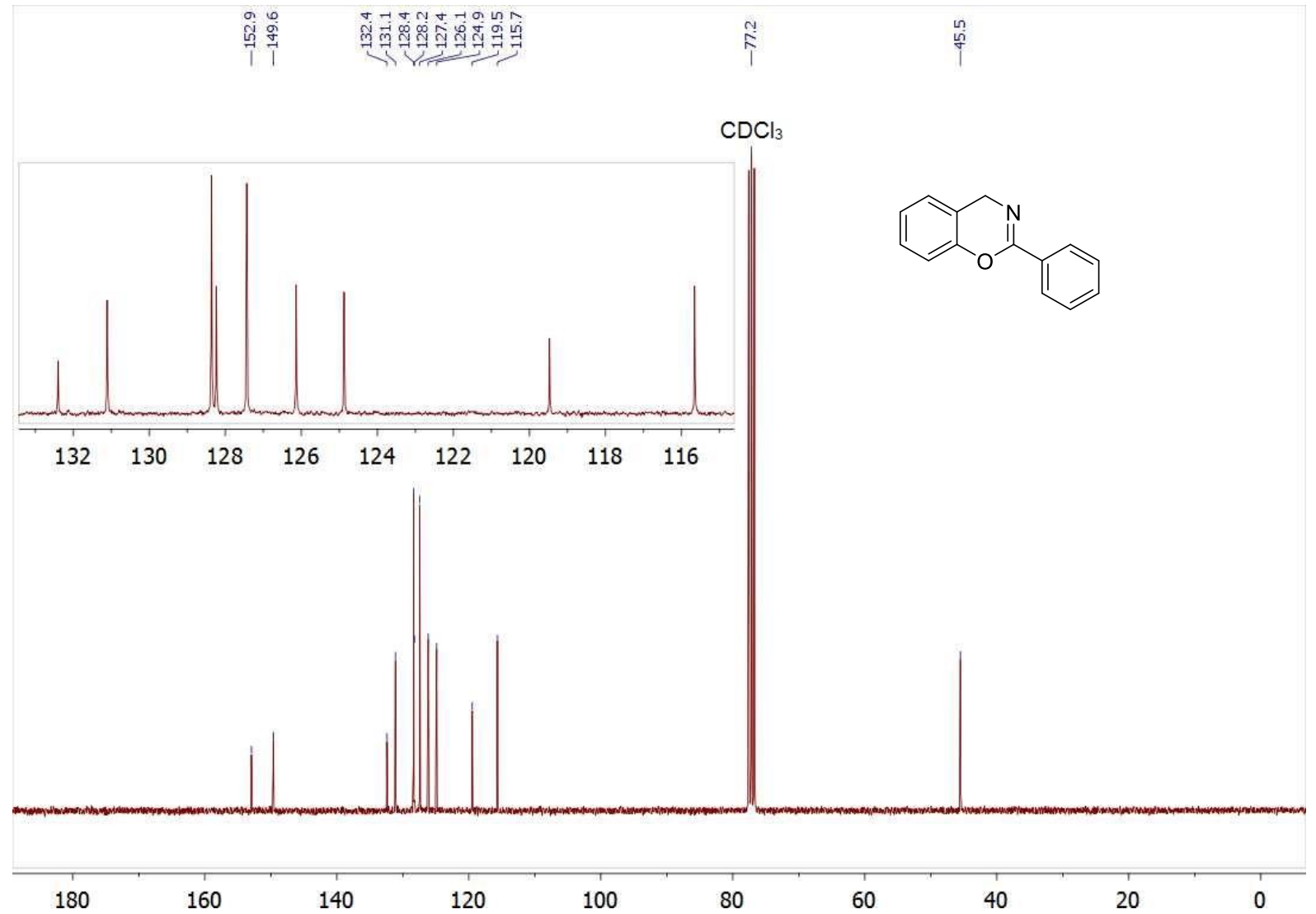

Figure S11: ${ }^{13} \mathrm{C}\left\{{ }^{1} \mathrm{H}\right\}$ NMR (75 $\mathrm{MHz}, \mathrm{CDCl}_{3}$ ) of 2-Phenyl-4H-1,3-benzoxazine 


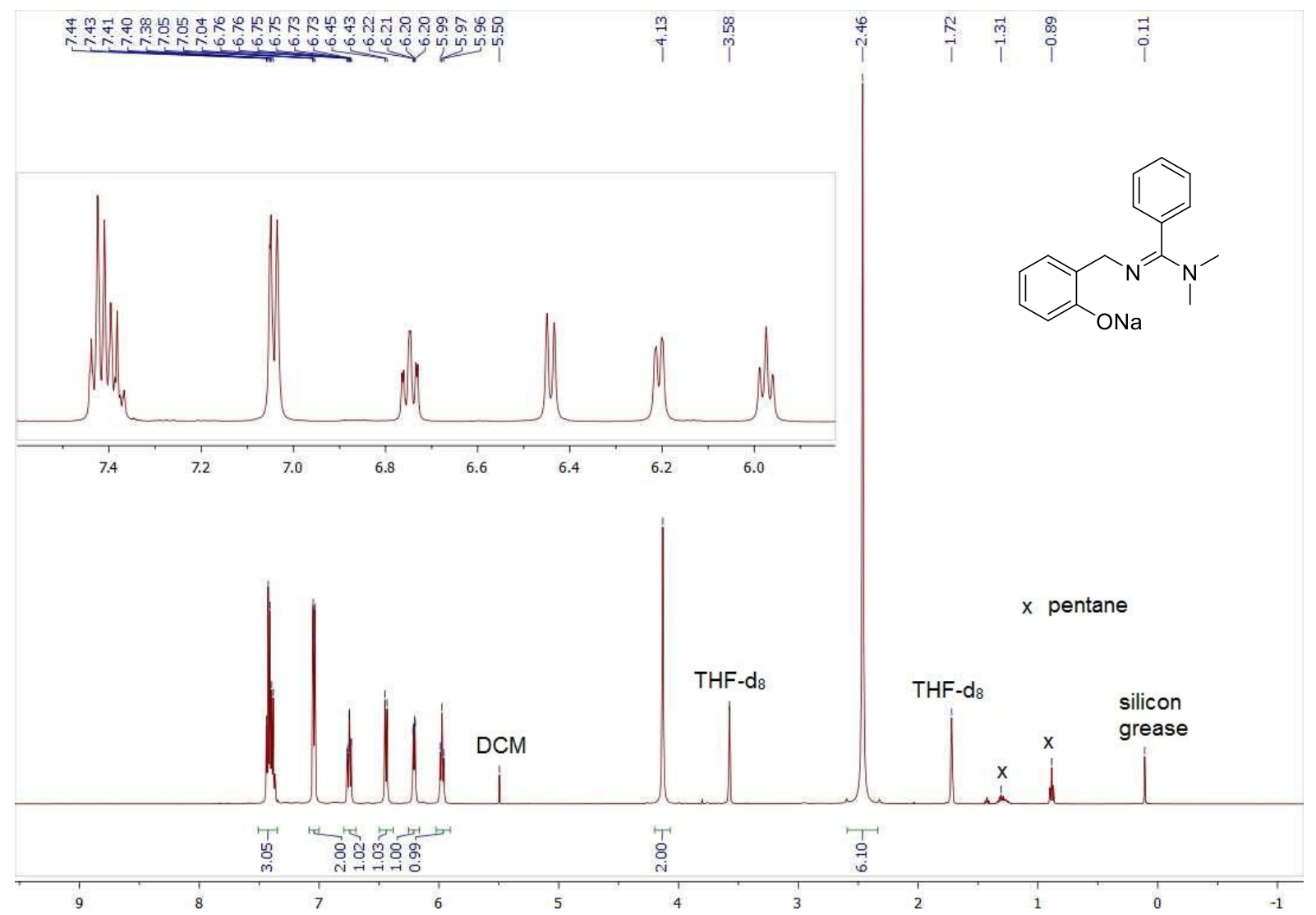

Figure S12: ${ }^{1} \mathrm{H}$ NMR $\left(500 \mathrm{MHz}, \mathrm{THF}-\mathrm{d}_{8}\right)$ of L1Na

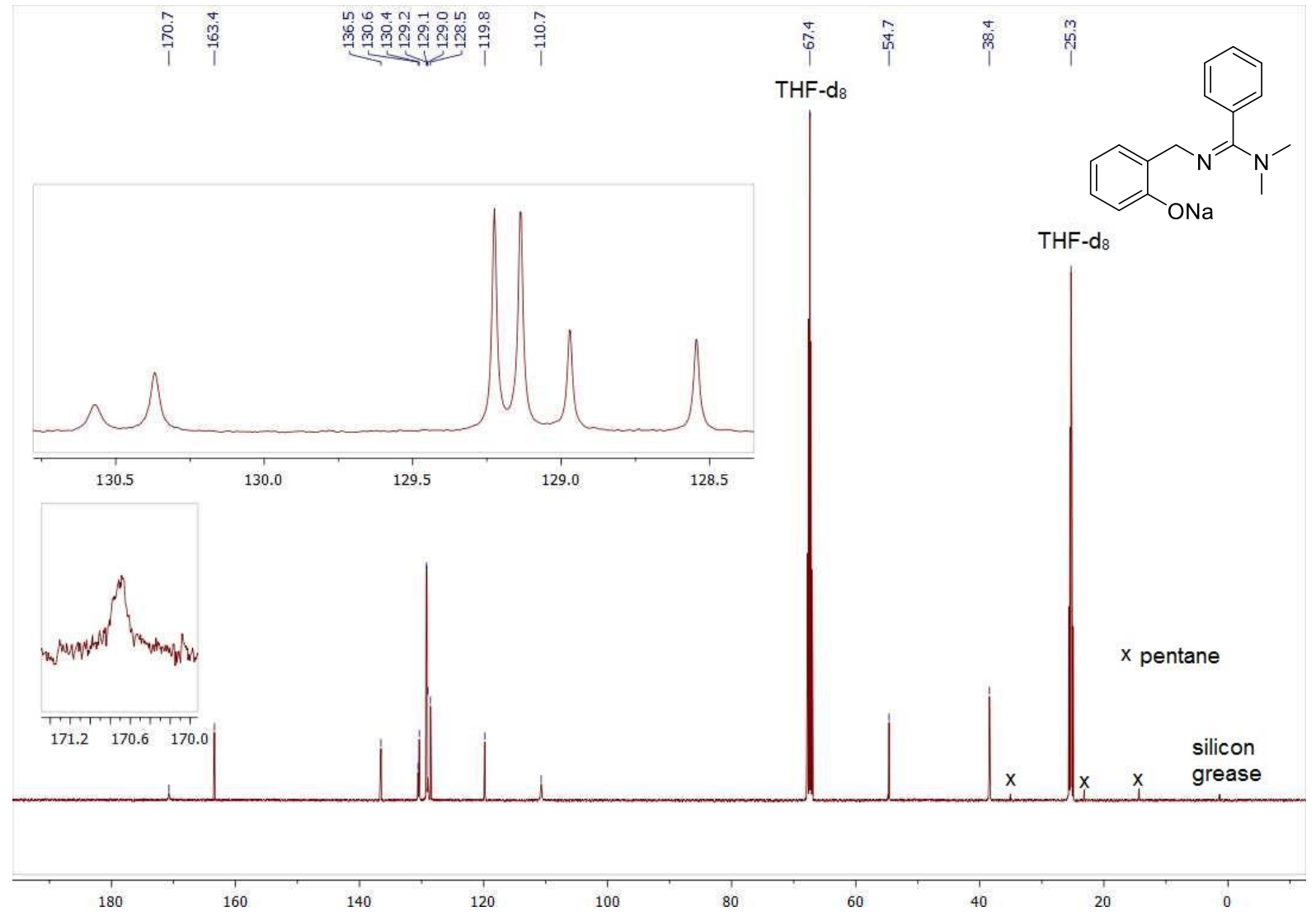

Figure S13: ${ }^{13} \mathrm{C}\left\{{ }^{1} \mathrm{H}\right\}$ NMR $\left(126 \mathrm{MHz}, \mathrm{THF}-\mathrm{d}_{8}\right)$ of L1Na 


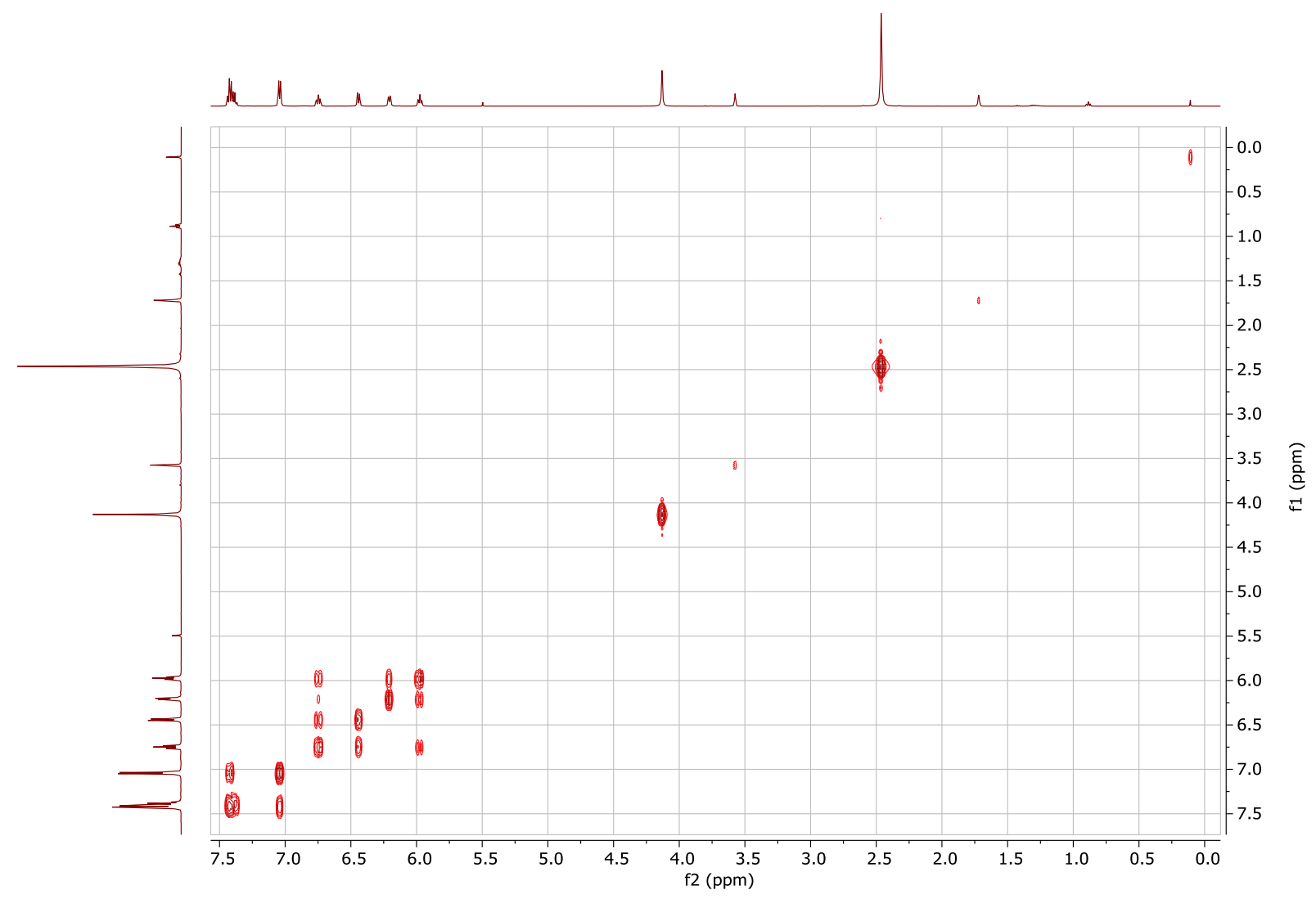

Figure S14: ${ }^{1} \mathrm{H}^{1} \mathrm{H}$ COSY (500 MHz, THF-d8) of L1Na

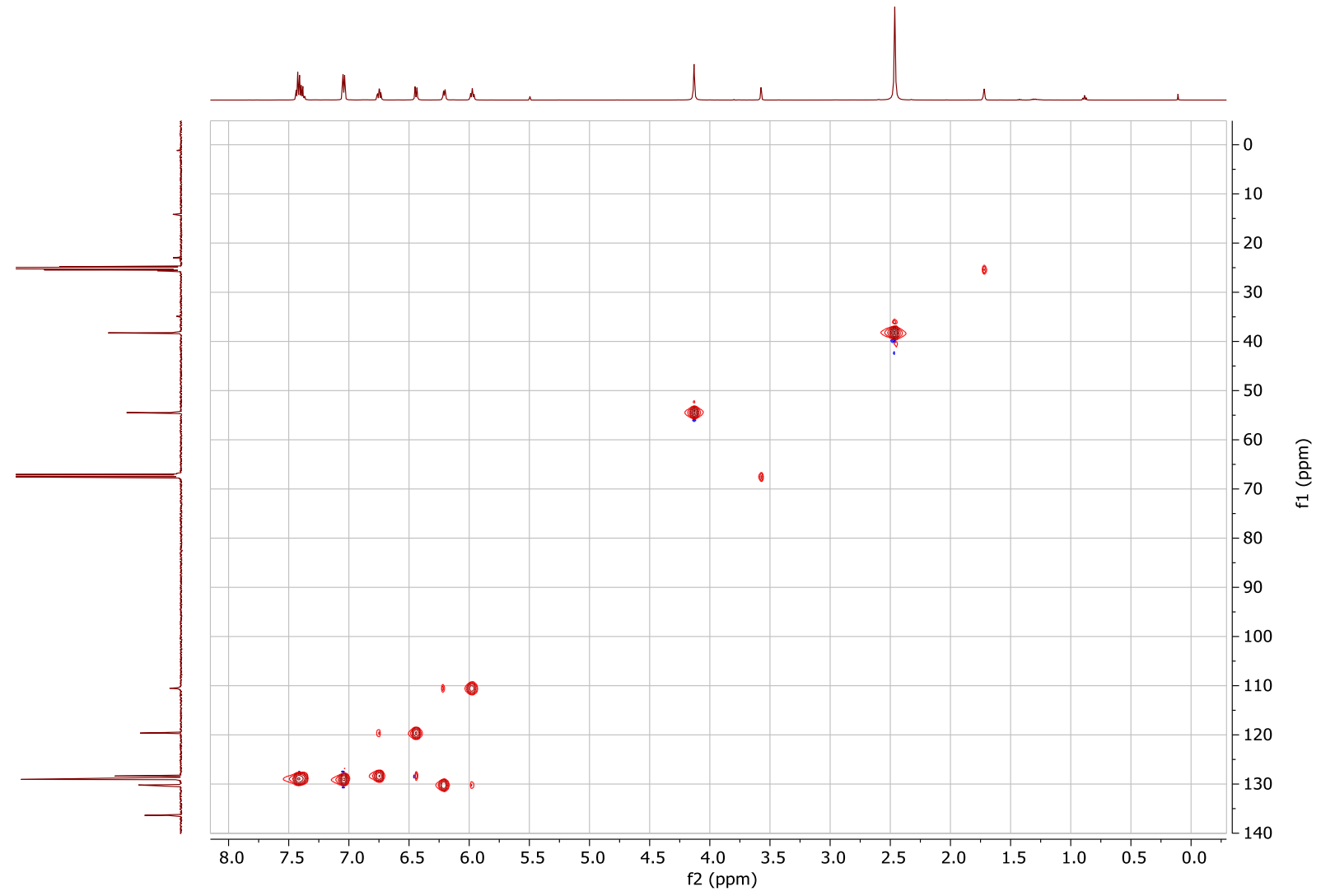

Figure S15: ${ }^{1} \mathrm{H}^{13} \mathrm{C}$ HSQC (500 MHz, $\left.126 \mathrm{MHz}, \mathrm{THF}-\mathrm{d}_{8}\right)$ of L1Na 


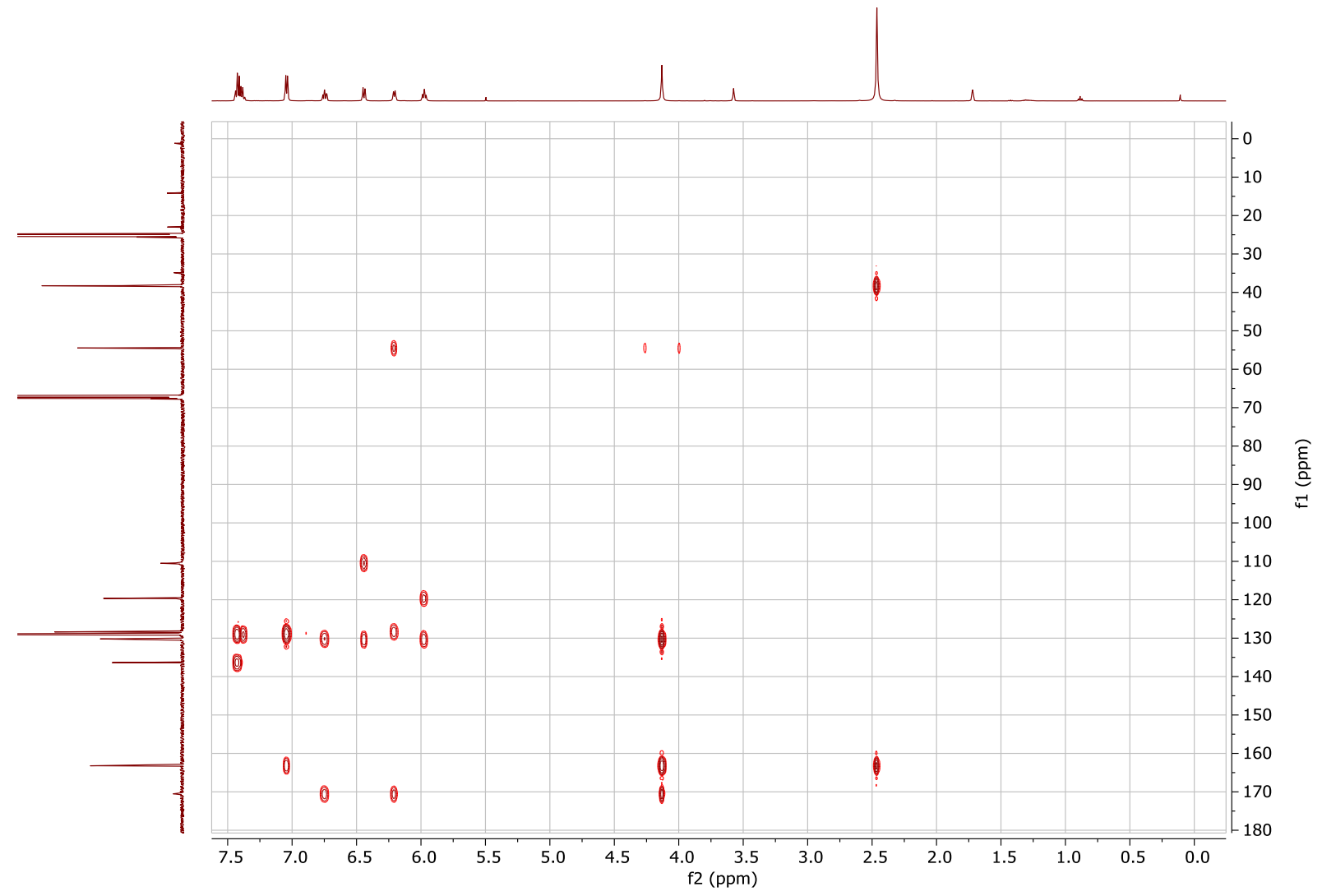

Figure S16: ${ }^{1} \mathrm{H}^{13} \mathrm{C}$ HMBC (500 MHz, $126 \mathrm{MHz}$, THF-d8) of L1Na

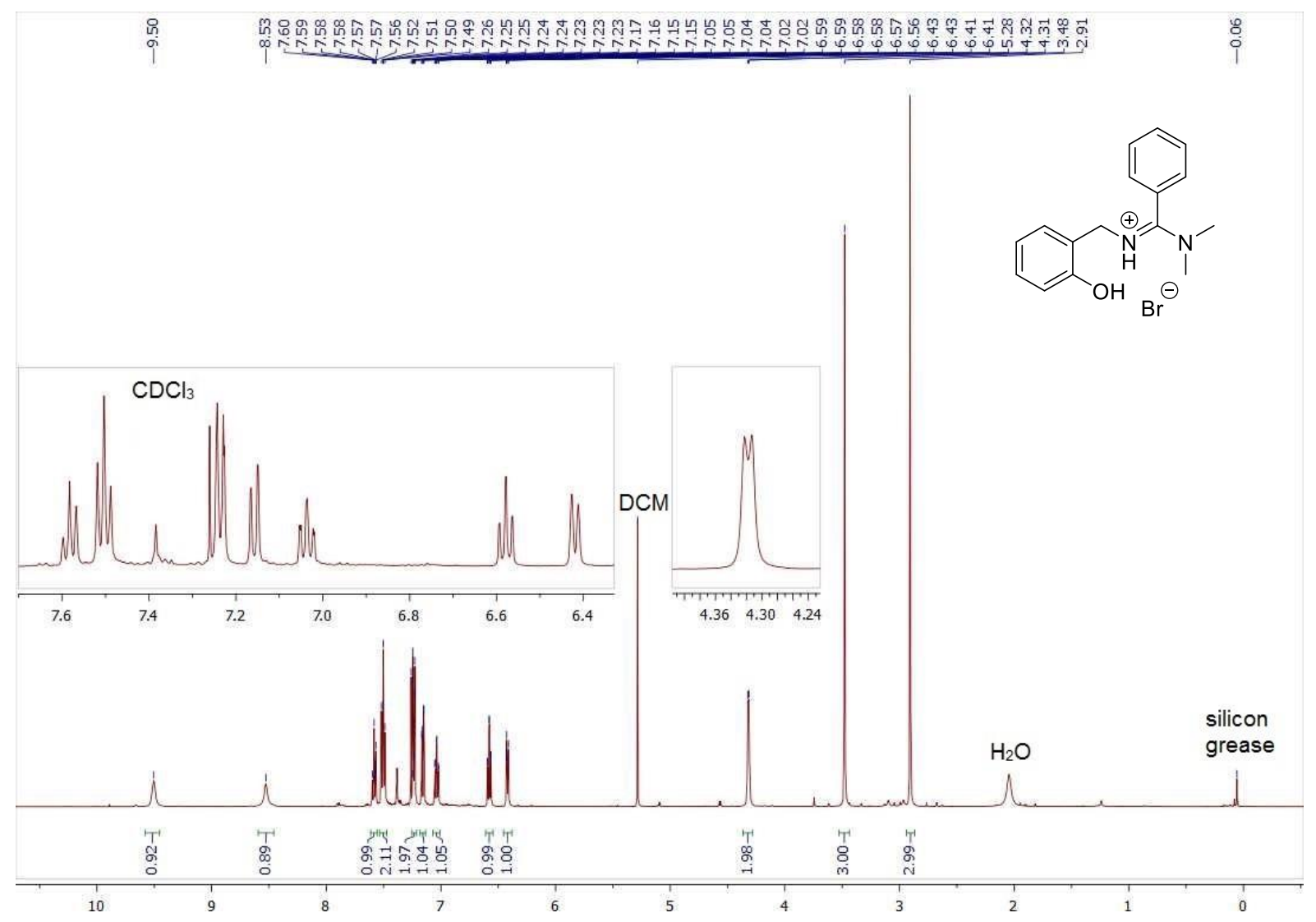

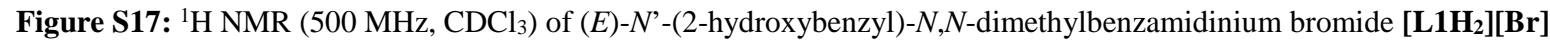



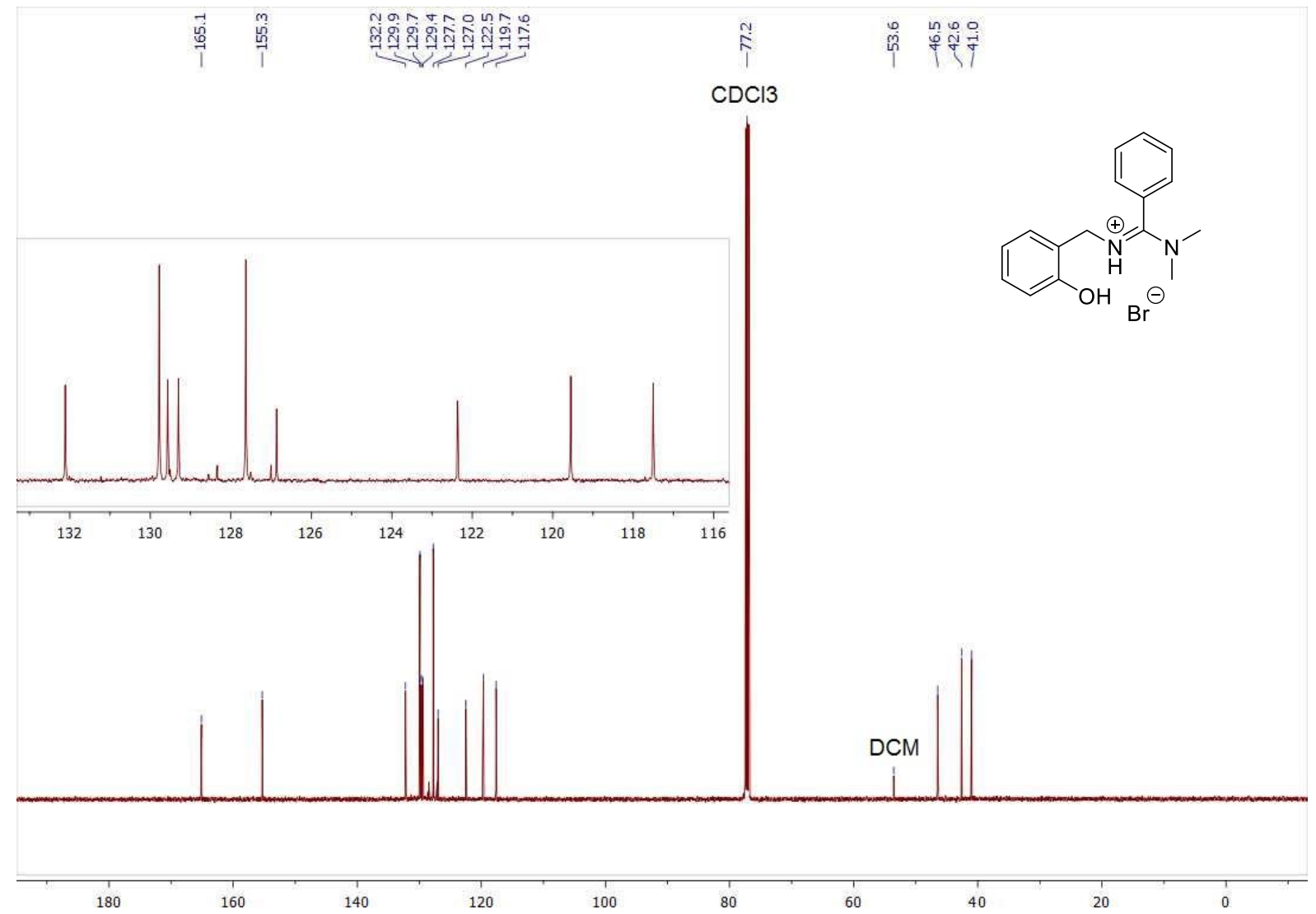

Figure S18: ${ }^{13} \mathrm{C}\left\{{ }^{1} \mathrm{H}\right\}$ NMR $\left(126 \mathrm{MHz}, \mathrm{CDCl}_{3}\right)$ of $(E)-N$ '-(2-hydroxybenzyl)- $N, N$-dimethylbenzamidinium bromide $\left[\mathrm{LLH}_{2}\right][\mathrm{Br}]$

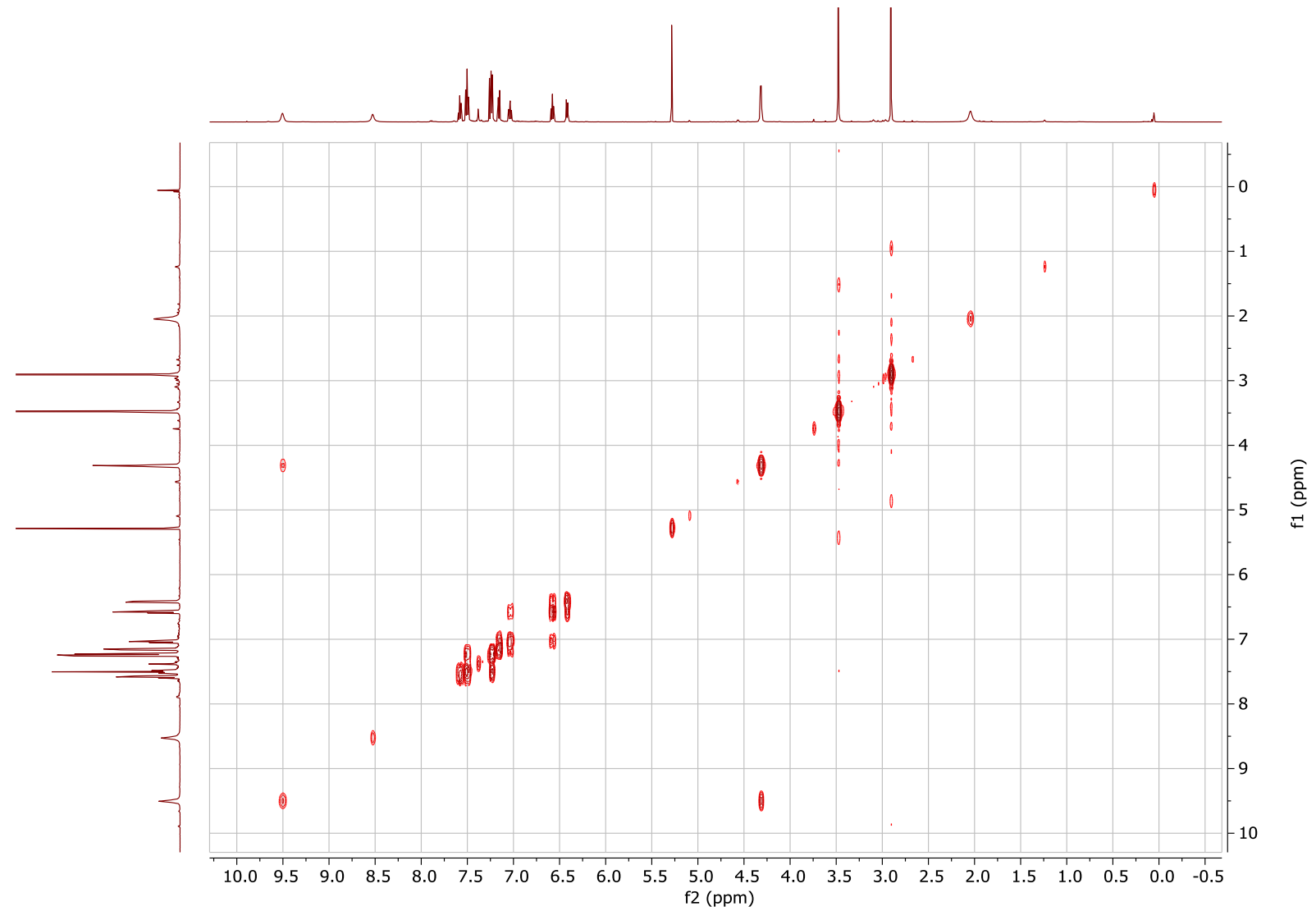

Figure S19: ${ }^{1} \mathrm{H}{ }^{1} \mathrm{H}$ COSY $\left(500 \mathrm{MHz}, \mathrm{CDCl}_{3}\right)$ of (E)- $N$ '-(2-hydroxybenzyl)- $N, N$-dimethylbenzamidinium bromide [ $\left.\mathbf{L 1} \mathbf{H}_{2}\right][\mathbf{B r}]$ 


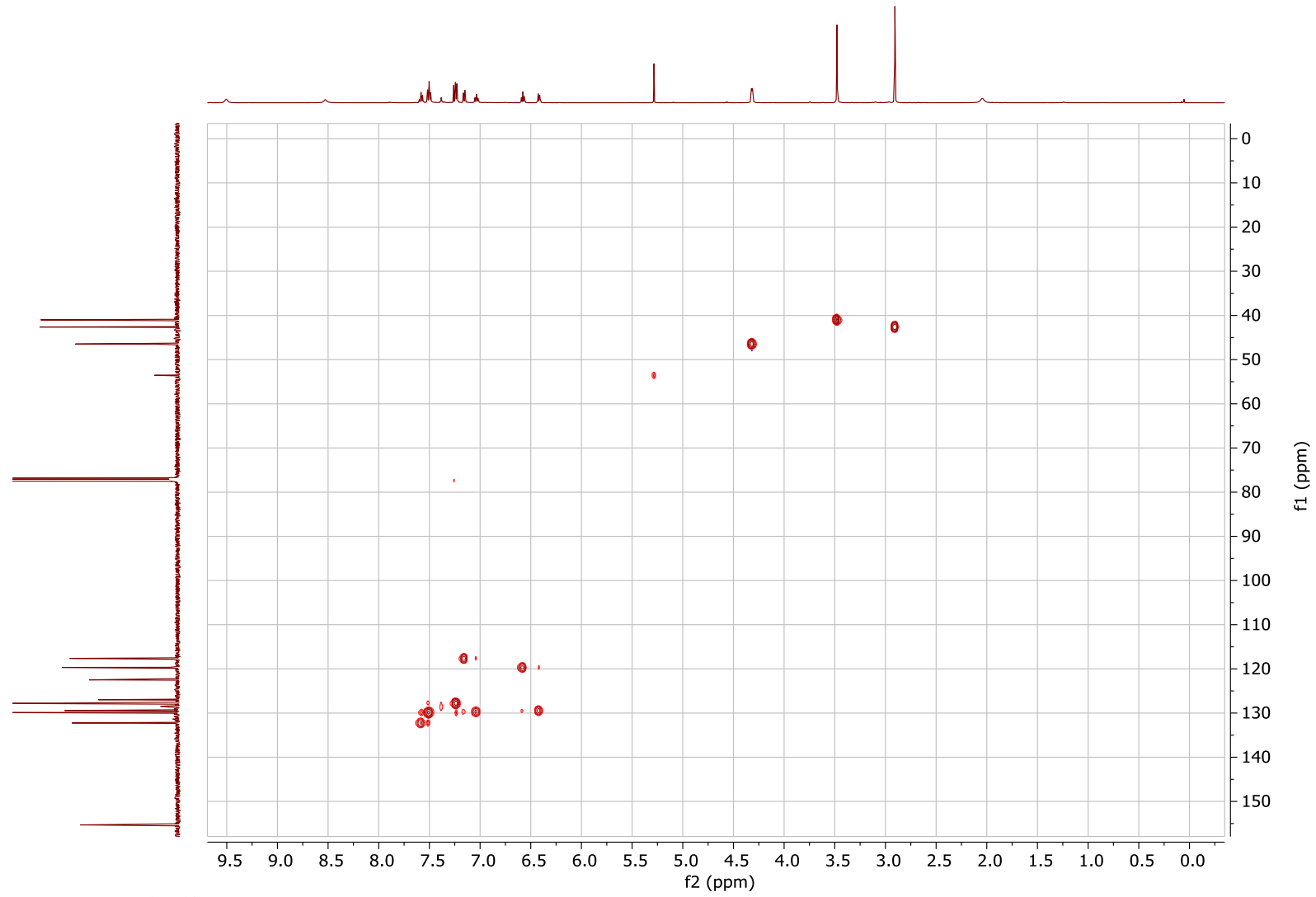

Figure S20: ${ }^{1} \mathrm{H}^{13} \mathrm{C}$ HSQC $\left(500 \mathrm{MHz}, 126 \mathrm{MHz}, \mathrm{CDCl}_{3}\right.$ ) of (E)- $N$ '-(2-hydroxybenzyl)- $N, N$-dimethylbenzamidinium bromide $\left[\mathbf{L} 1 \mathrm{H}_{2}\right][\mathrm{Br}]$

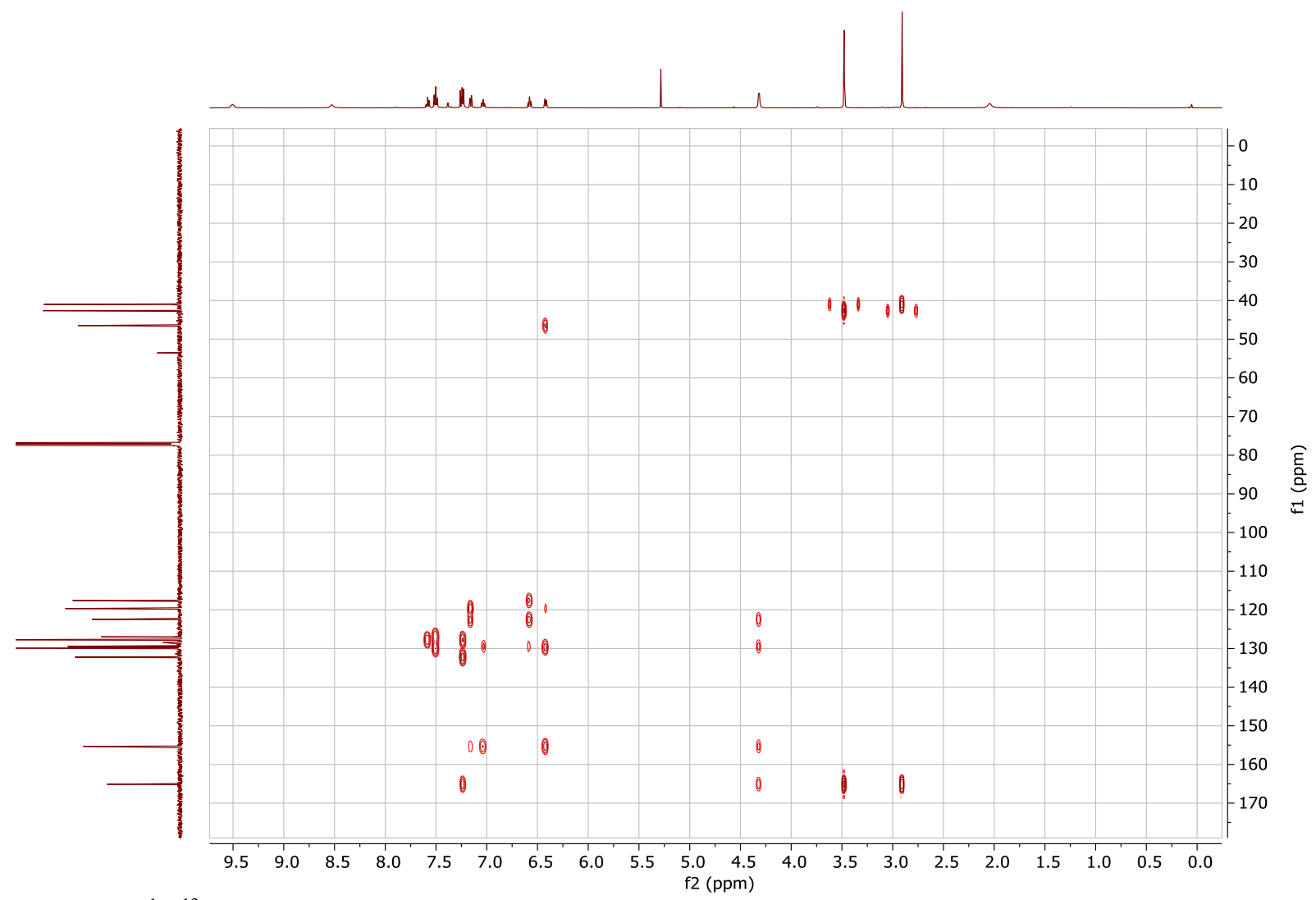

Figure S21: ${ }^{1} \mathrm{H}^{13} \mathrm{C} \mathrm{HMBC}\left(500 \mathrm{MHz}, 126 \mathrm{MHz}, \mathrm{CDCl}_{3}\right)$ of $(E)-N$ '-(2-hydroxybenzyl)- $N, N$-dimethylbenzamidinium bromide $\left[\mathbf{L 1} \mathbf{H}_{2}\right][\mathbf{B r}]$ 


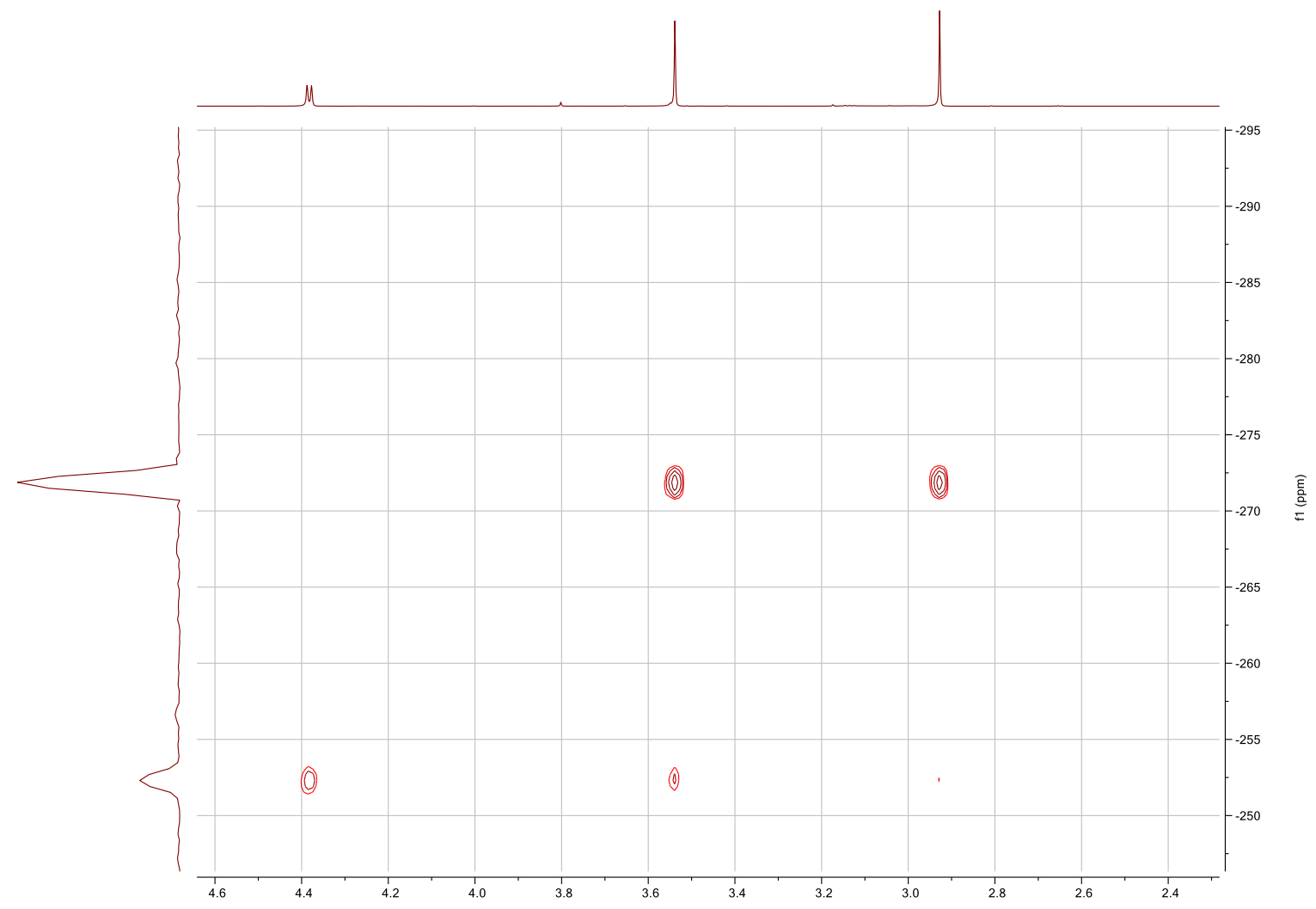

Figure S22: ${ }^{1} \mathrm{H}{ }^{15} \mathrm{~N}$ HMBC $\left(\mathrm{CD}_{2} \mathrm{Cl}_{2}, 600.23 \mathrm{MHz} / 43.3 \mathrm{MHz}\right)$ of $(E)-N$ '-(2-hydroxybenzyl)- $N, N$-dimethylbenzamidinium bromide $\left[\mathbf{L 1} \mathbf{H}_{2}\right][\mathbf{B r}]$

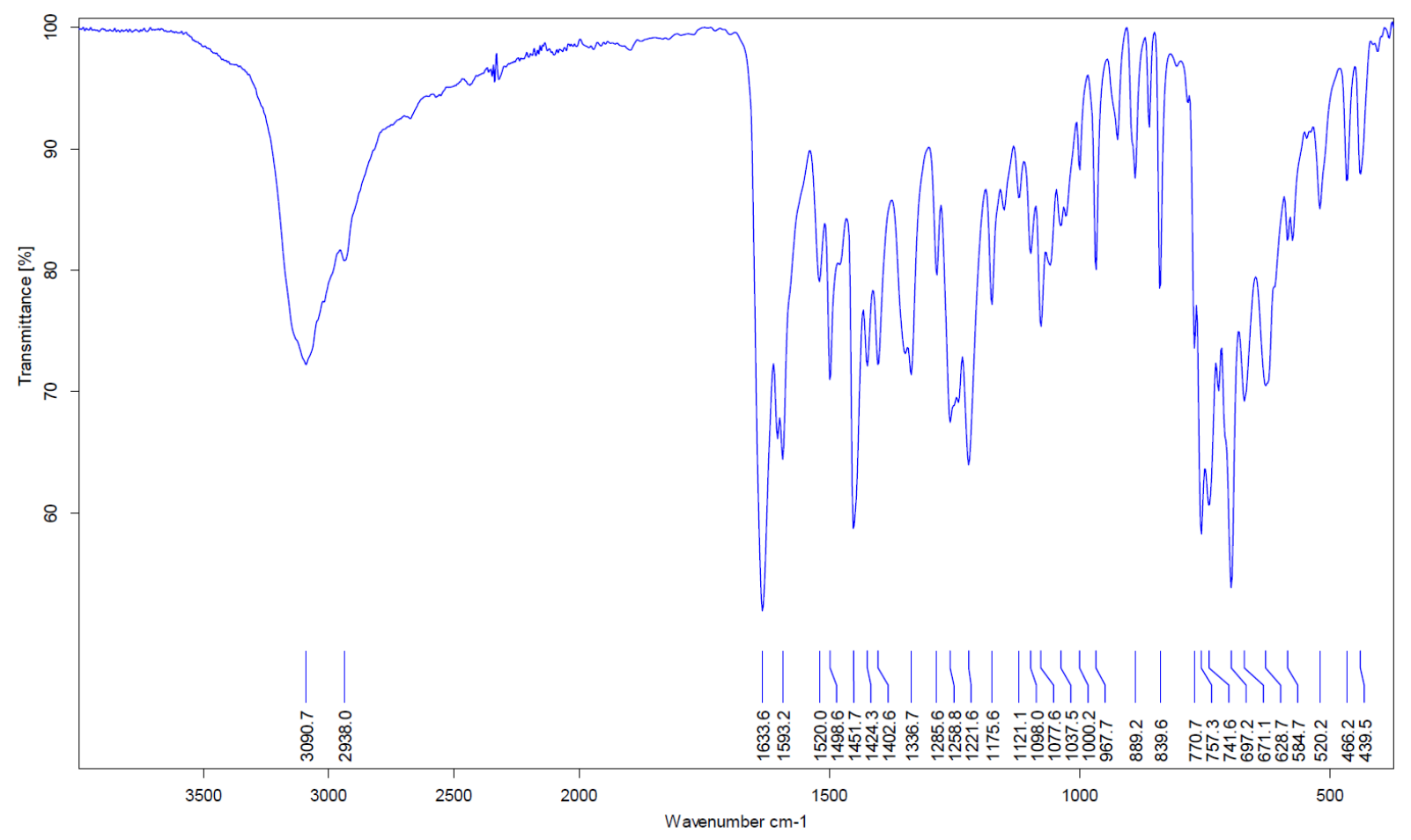

Figure S23: ATR-FT-IR spectrum of $(E)-N^{\prime}$-(2-hydroxybenzyl)- $N, N$-dimethylbenzamidinium bromide [L1H2] [Br] 


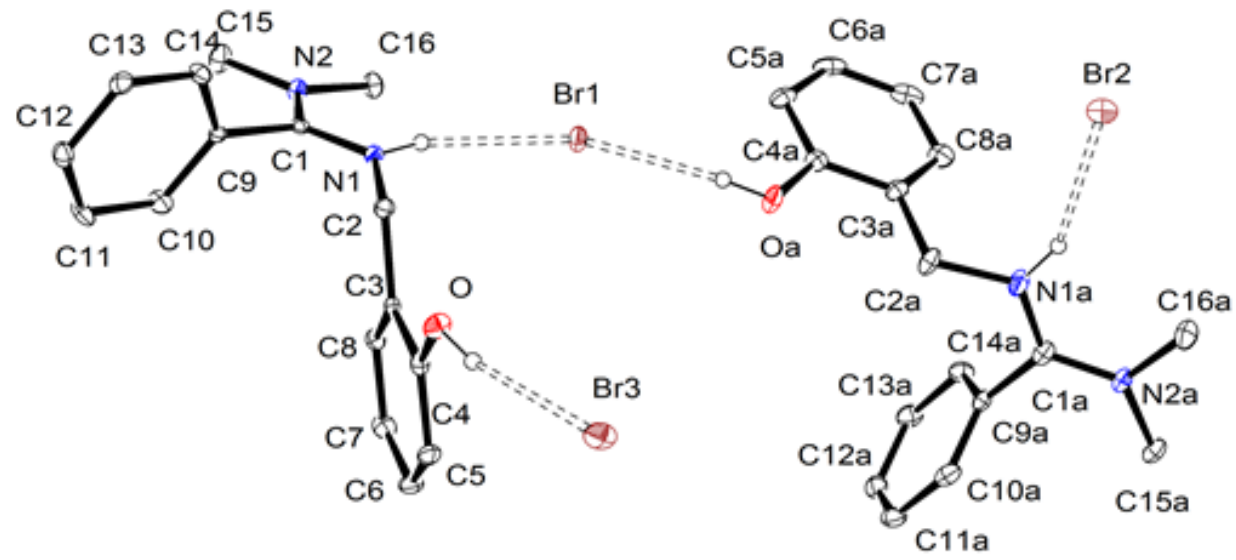

Figure S24: Crystal structure of $(E)-N^{\prime}-(2$-hydroxybenzyl)- $N, N$-dimethylbenzamidinium bromide [L1H 2$][\mathbf{B r}]$

Table S1: Crystal data and structure refinement of $\left[\mathbf{L 1} \mathbf{H}_{2}\right][\mathbf{B r}]$

\begin{tabular}{|c|c|}
\hline Identification code & $\mathbf{L}_{1} H_{2} \mathbf{B r}$ \\
\hline Empirical formula & $\mathrm{C}_{16} \mathrm{H}_{19} \mathrm{BrN}_{2} \mathrm{O}$ \\
\hline Formula weight & 335.24 \\
\hline Temperature/K & 115 \\
\hline Crystal system & triclinic \\
\hline Space group & $\mathrm{P}-1$ \\
\hline $\mathrm{a} / \AA$ & $9.1905(4)$ \\
\hline $\mathrm{b} / \AA$ & $12.2461(5)$ \\
\hline$c / \AA$ & $14.6440(6)$ \\
\hline$\alpha /{ }^{\circ}$ & $73.983(2)$ \\
\hline$\beta /{ }^{\circ}$ & $77.158(2)$ \\
\hline$\gamma /{ }^{\circ}$ & $77.693(2)$ \\
\hline Volume $/ \AA^{3}$ & $1524.14(11)$ \\
\hline $\mathrm{Z}$ & 4 \\
\hline$\rho_{\text {calc }} \mathrm{g} / \mathrm{cm}^{3}$ & 1.461 \\
\hline$\mu / \mathrm{mm}^{-1}$ & 2.695 \\
\hline $\mathrm{F}(000)$ & 688.0 \\
\hline Crystal size $/ \mathrm{mm}^{3}$ & $0.5 \times 0.5 \times 0.025$ \\
\hline Radiation & $\operatorname{MoK} \alpha(\lambda=0.71073)$ \\
\hline \multicolumn{2}{|c|}{$2 \Theta$ range for data collection $/{ }^{\circ} 5.878$ to 55.066} \\
\hline Index ranges & $-11 \leq \mathrm{h} \leq 11,-15 \leq \mathrm{k} \leq 15,-18 \leq 1 \leq 19$ \\
\hline Reflections collected & 41247 \\
\hline Independent reflections & $7000\left[R_{\text {int }}=0.0552, R_{\text {sigma }}=0.0444\right]$ \\
\hline Data/restraints/parameters & $7000 / 0 / 370$ \\
\hline Goodness-of-fit on $\mathrm{F}^{2}$ & 1.051 \\
\hline Final $R$ indexes $[I>=2 \sigma(I)]$ & $\mathrm{R}_{1}=0.0349, \mathrm{wR}_{2}=0.0754$ \\
\hline Final R indexes [all data] & $\mathrm{R}_{1}=0.0541, \mathrm{wR}_{2}=0.0825$ \\
\hline \multicolumn{2}{|c|}{ Largest diff. peak/hole / e $\AA^{-3} 0.51 /-0.63$} \\
\hline CCDC & 1868743 \\
\hline
\end{tabular}




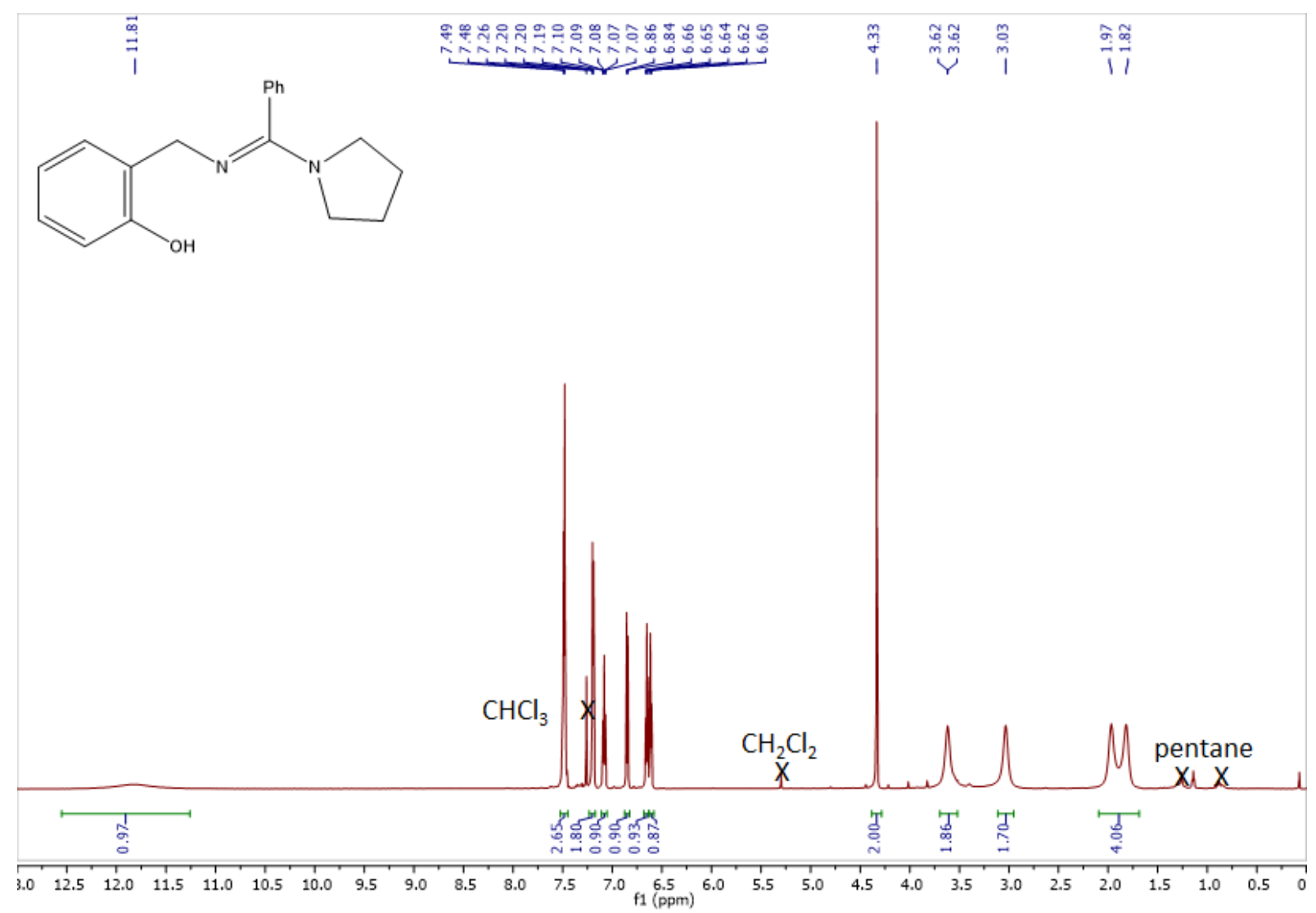

Figure S25: ${ }^{1} \mathrm{H}$ NMR $\left(500 \mathrm{MHz}, \mathrm{CDCl}_{3}\right)$ of $(E)-N$ '-(2-hydroxybenzyl)pyrrolidinylbenzamidine $\mathbf{L 2 H}$

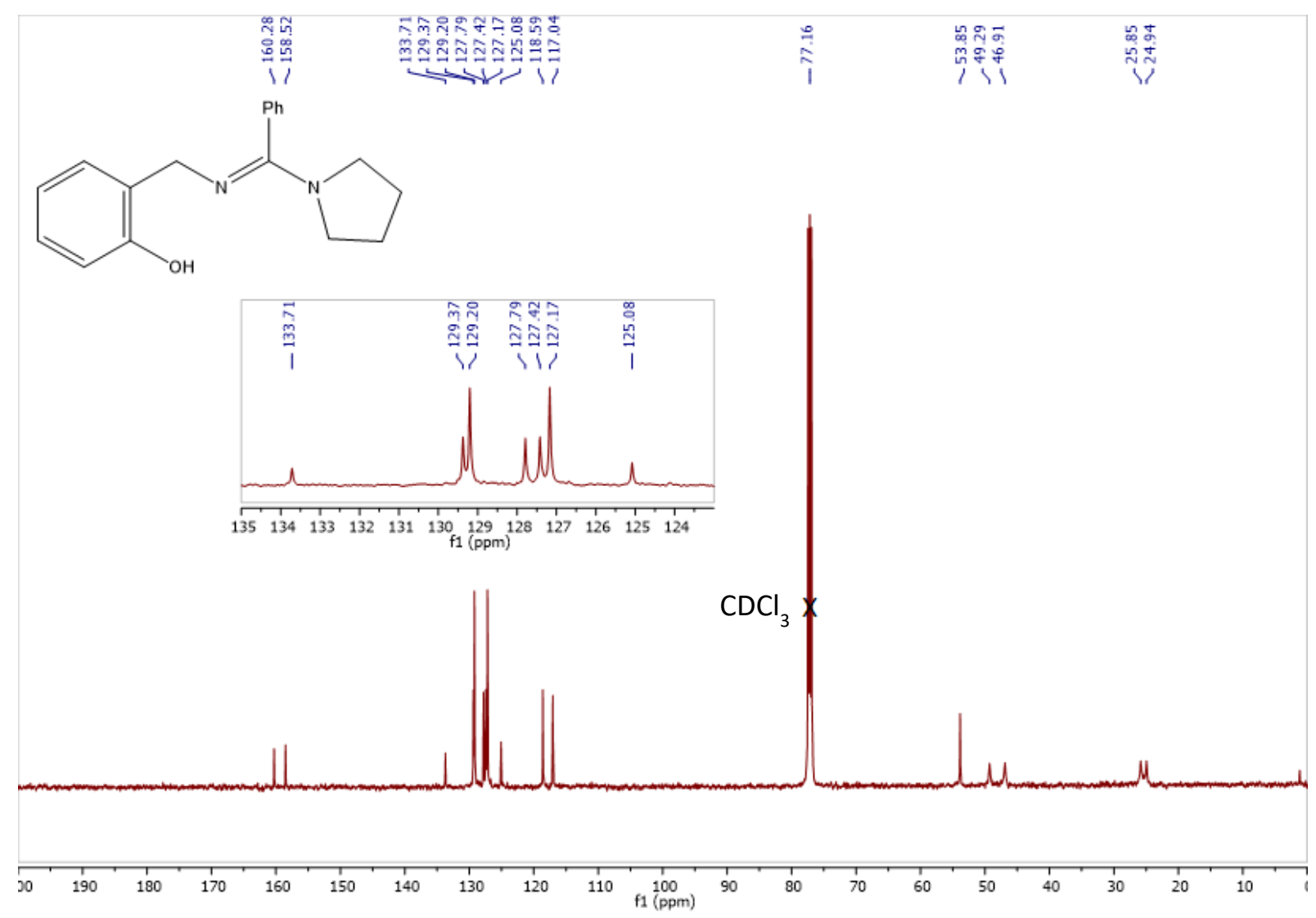

Figure S26: ${ }^{13} \mathrm{C}\left\{{ }^{1} \mathrm{H}\right\}$ NMR $\left(126 \mathrm{MHz}, \mathrm{CDCl}_{3}\right)$ of $(E)-N$ '-(2-hydroxybenzyl)pyrrolidinylbenzamidine $\mathbf{L 2 H}$ 


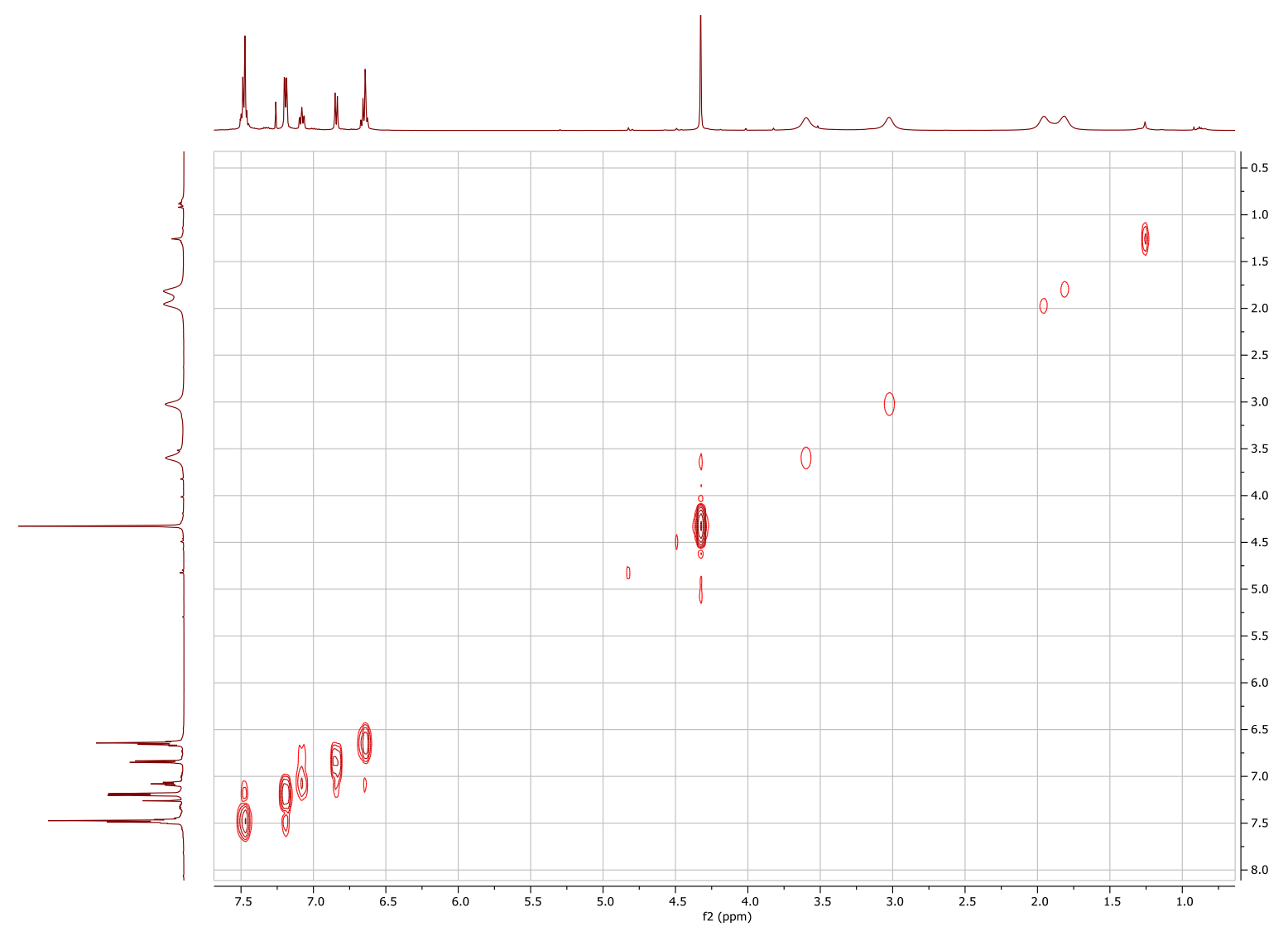

Figure S27: ${ }^{1} \mathrm{H}{ }^{1} \mathrm{H} \operatorname{COSY}\left(500 \mathrm{MHz}, \mathrm{CDCl}_{3}\right)$ of $(E)-N$ '-(2-hydroxybenzyl)pyrrolidinylbenzamidine $\mathbf{L 2 H}$

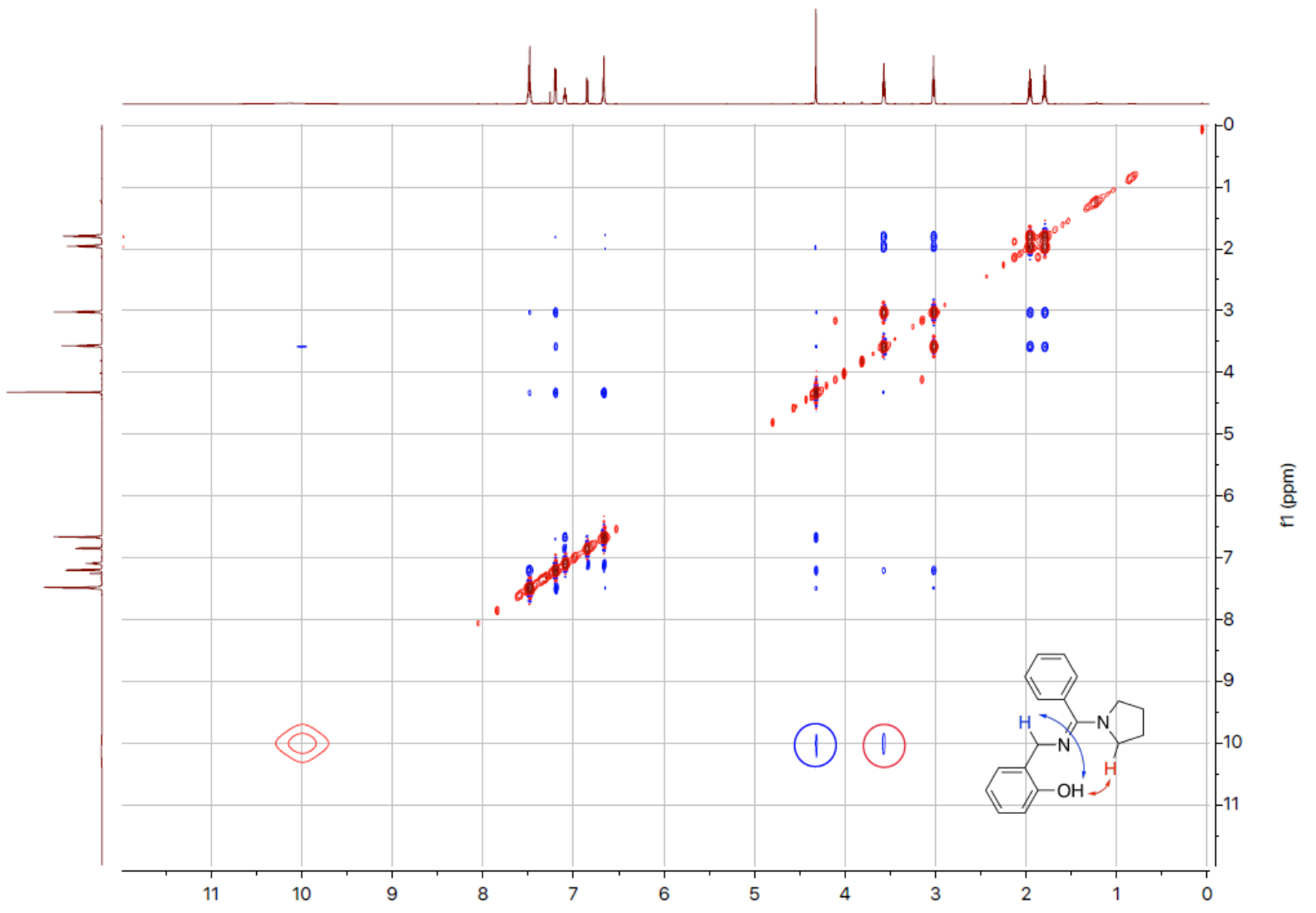

Figure S28: ${ }^{1} \mathrm{H}{ }^{1} \mathrm{H}$ NOESY $\left(500 \mathrm{MHz}, 250 \mathrm{~K}, \mathrm{CDCl}_{3}\right)$ of $(E)-N^{\prime}$-(2-hydroxybenzyl)pyrrolidinylbenzamidine $\mathbf{L 2 H}$ 


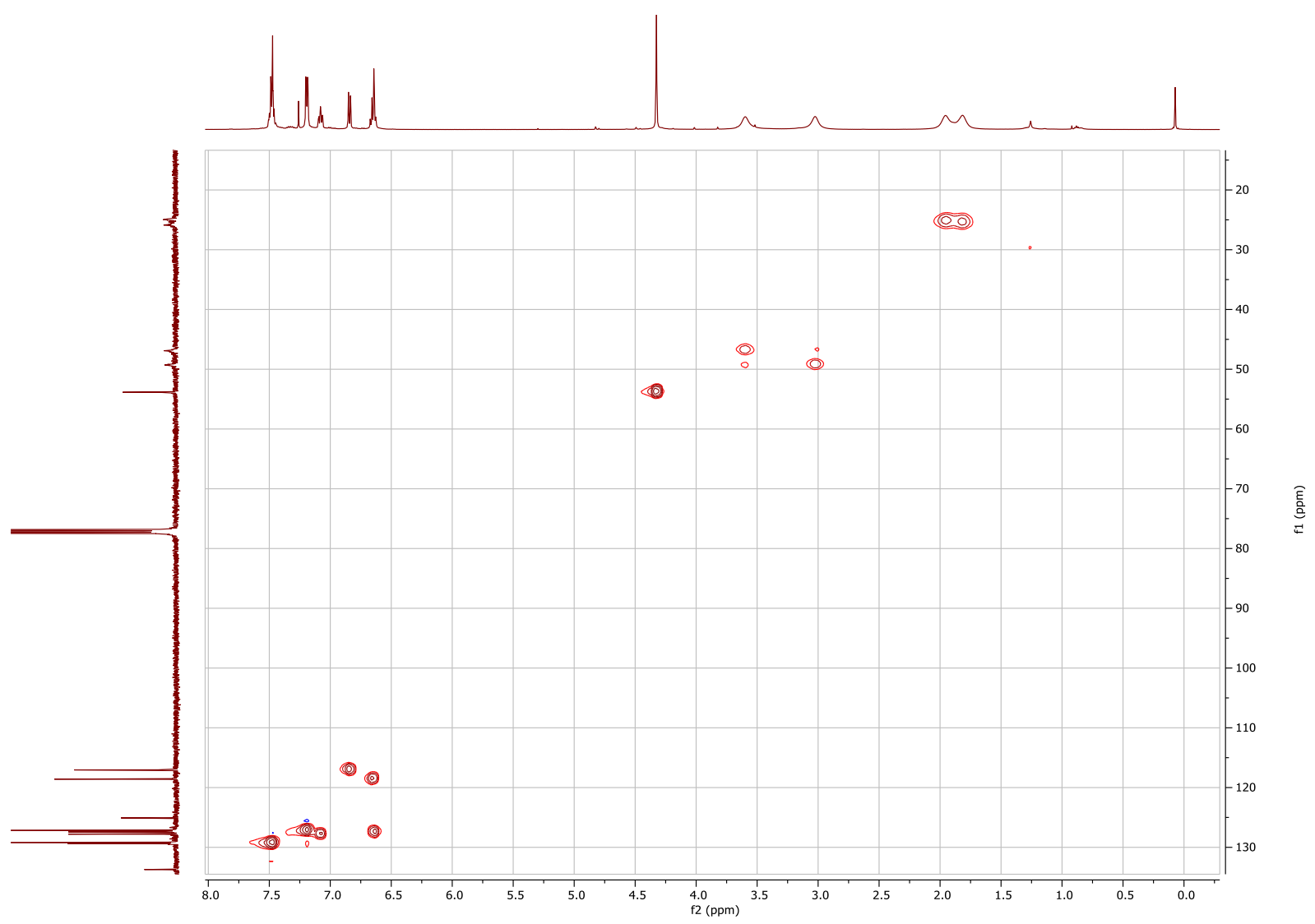

Figure S29: ${ }^{1} \mathrm{H}^{13} \mathrm{C}$ HSQC (500 MHz / $126 \mathrm{MHz}, \mathrm{CDCl}_{3}$ ) of (E)- $N$ '-(2-hydroxybenzyl)pyrrolidinylbenzamidine $\mathbf{L 2 H}$

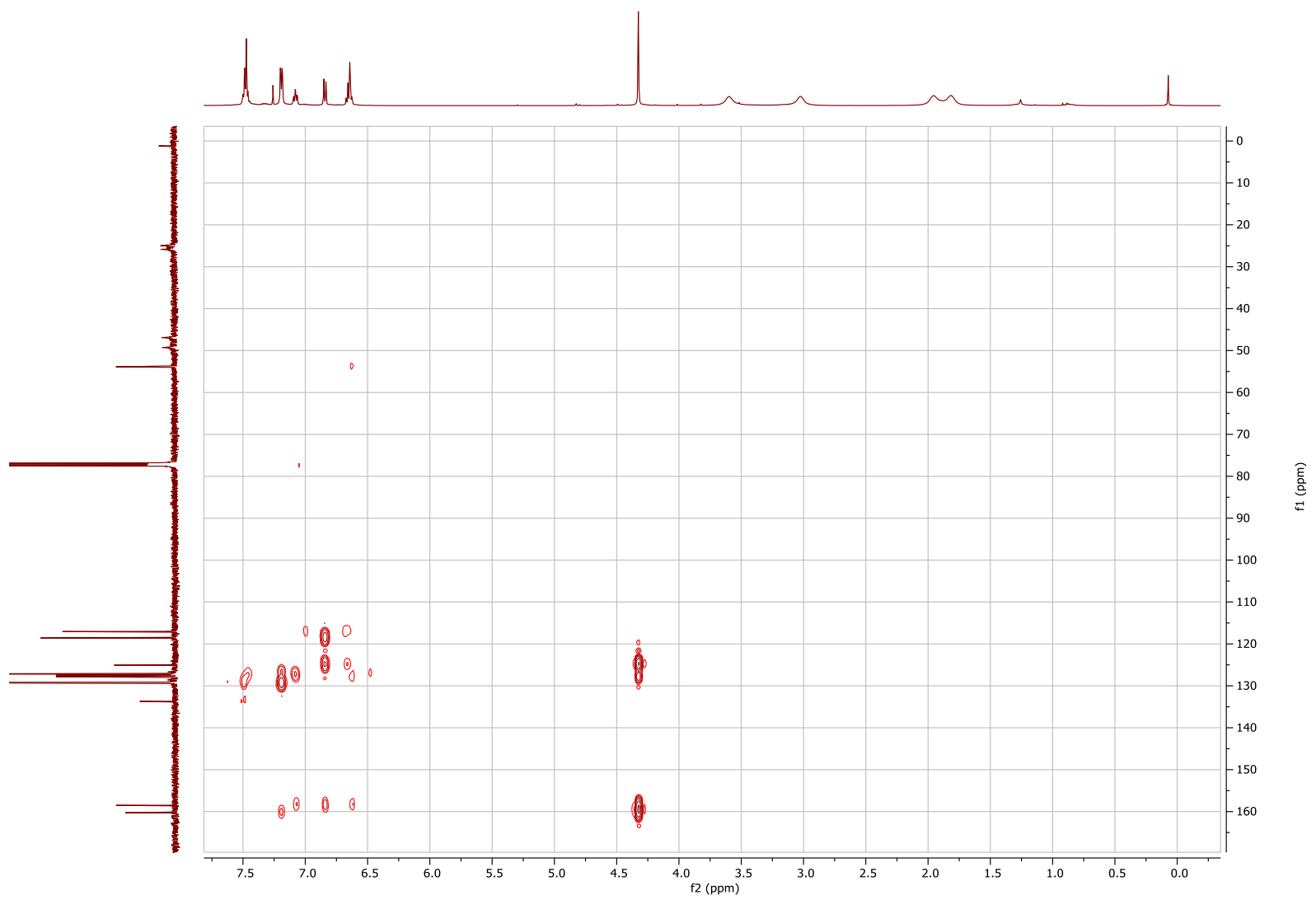

Figure S30: ${ }^{1} \mathrm{H}^{13} \mathrm{C} \mathrm{HMBC}\left(500 \mathrm{MHz} / 126 \mathrm{MHz}, \mathrm{CDCl}_{3}\right)$ of $(E)-N^{\prime}$-(2-hydroxybenzyl)pyrrolidinylbenzamidine $\mathbf{L 2 H}$

S17 


(250

Figure S31: VT ${ }^{1} \mathrm{H}$ NMR $\left(600 \mathrm{MHz}, \mathrm{CDCl}_{3}\right)$ of $(E)-N^{\prime}$-(2-hydroxybenzyl)pyrrolidinylbenzamidine L2H showing the pyrrolidine region of the ligand. Partial degradation of $\mathbf{L 2 H}$ to benzoxazine and pyrrolidine* occurred once the temperature raised $305 \mathrm{~K}$.

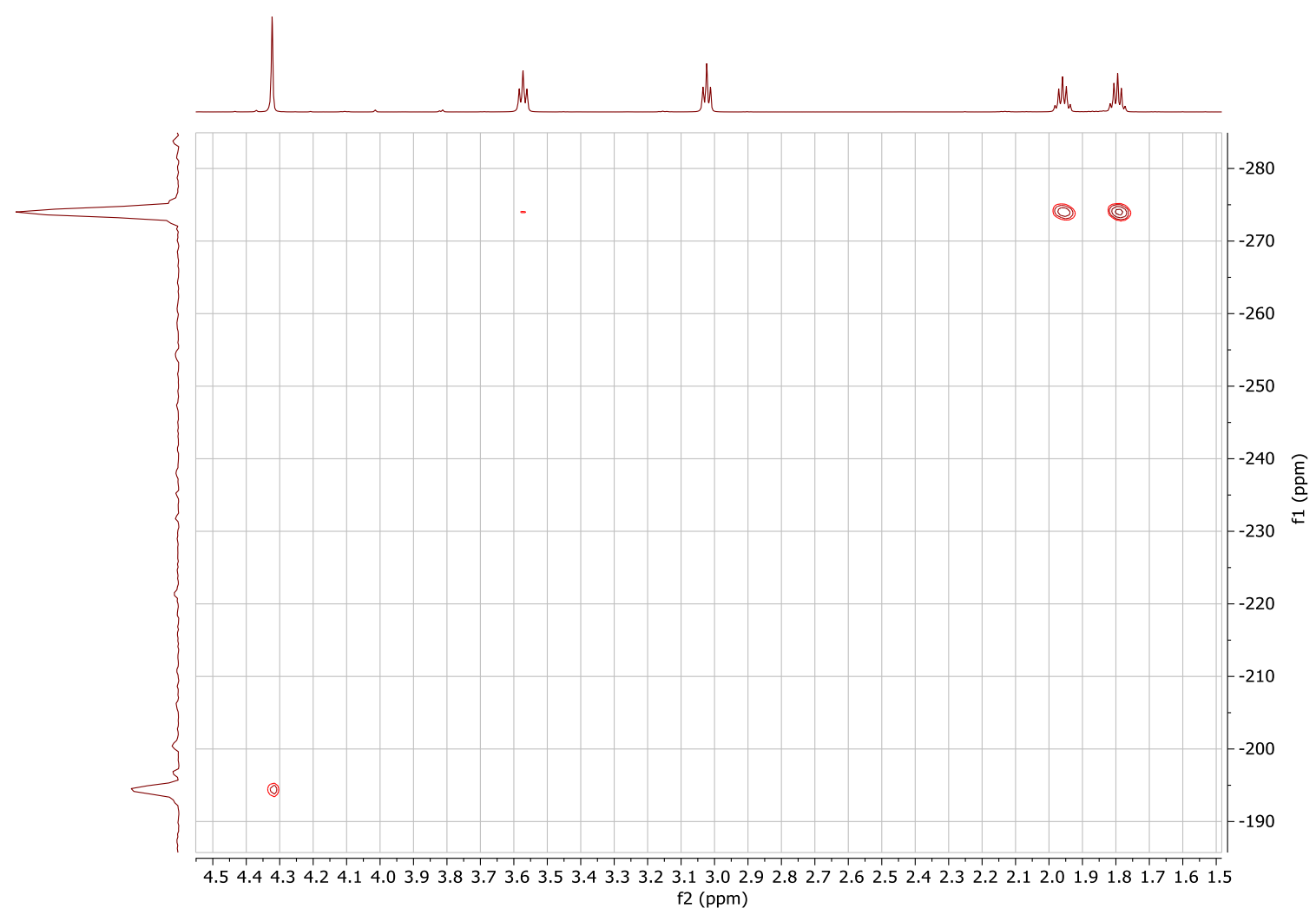

Figure S32: ${ }^{1} \mathrm{H}{ }^{15} \mathrm{~N}$ HMBC $\left(\mathrm{CDCl}_{3}, 250 \mathrm{~K}, 600.23 \mathrm{MHz} / 43.3 \mathrm{MHz}\right)$ of $(E)-N^{\prime}$-(2-hydroxybenzyl)pyrrolidinylbenzamidine L2H 


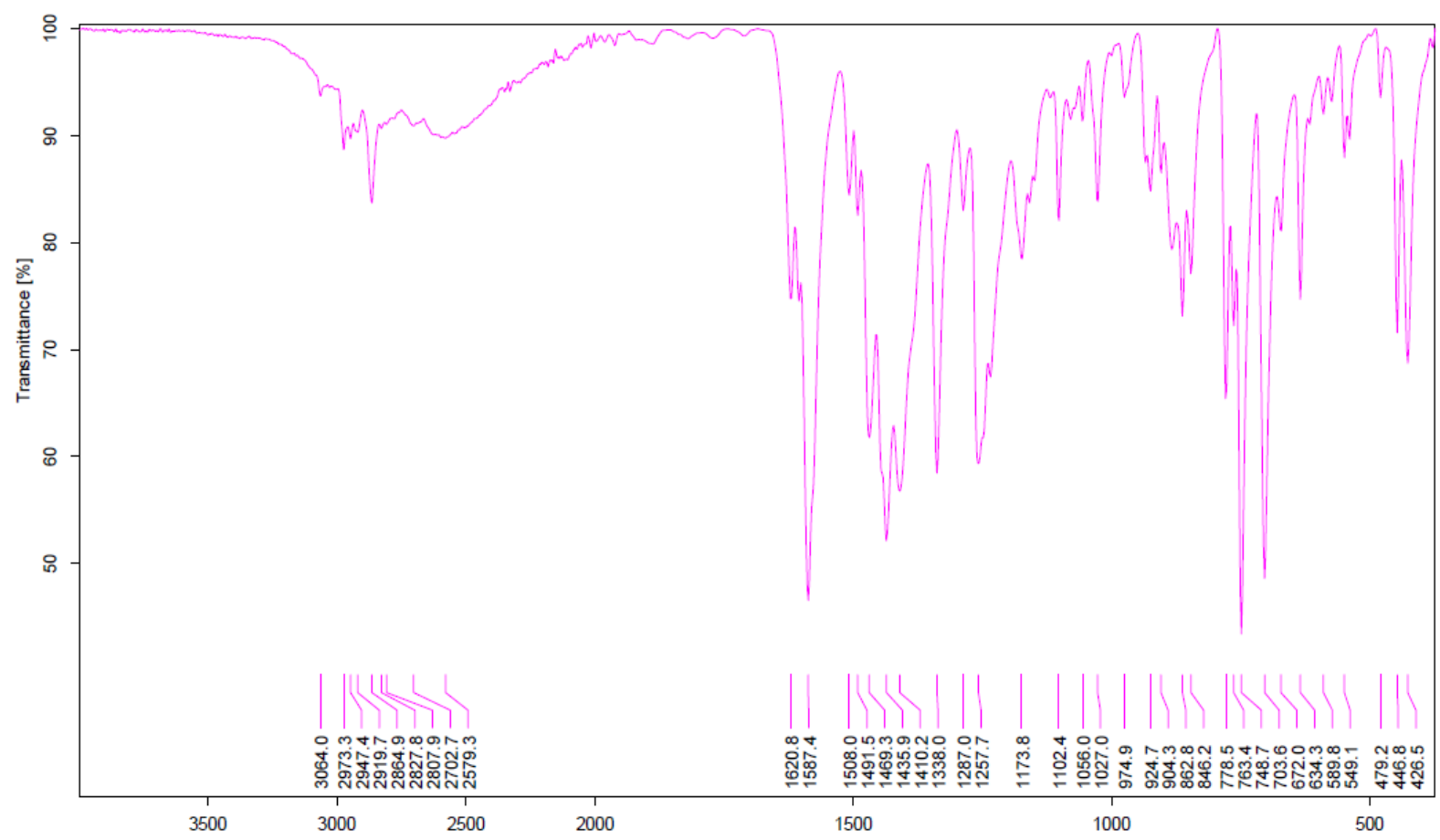

Figure S33: ATR-FT-IR spectrum of (E)-N'-(2-hydroxybenzyl)pyrrolidinylbenzamidine L2H

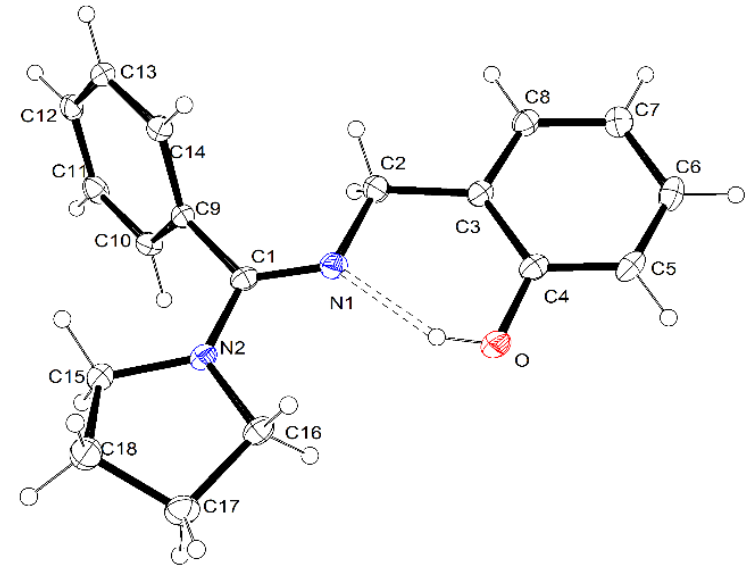

Figure S34: Crystal structure of (E)-N’-(2-hydroxybenzyl)pyrrolidinylbenzamidine L2H 
Table S2: Crystal data and structure refinement of (E)-N'-(2-hydroxybenzyl)pyrrolidinylbenzamidine L2H

\begin{tabular}{|c|c|}
\hline Identification code & L2H \\
\hline Empirical formula & $\mathrm{C}_{18} \mathrm{H}_{20} \mathrm{~N}_{2} \mathrm{O}$ \\
\hline Formula weight & 280.36 \\
\hline Temperature/K & 110 \\
\hline Crystal system & orthorhombic \\
\hline Space group & Pbca \\
\hline $\mathrm{a} / \AA$ & $11.2160(18)$ \\
\hline $\mathrm{b} / \AA$ & $15.574(3)$ \\
\hline $\mathrm{c} / \AA$ & $17.168(3)$ \\
\hline$\alpha /^{\circ}$ & 90 \\
\hline$\beta /{ }^{\circ}$ & 90 \\
\hline$\gamma /{ }^{\circ}$ & 90 \\
\hline Volume $/ \AA^{3}$ & 2999.0(9) \\
\hline $\mathrm{Z}$ & 8 \\
\hline$\rho_{\text {calc }} \mathrm{g} / \mathrm{cm}^{3}$ & 1.242 \\
\hline$\mu / \mathrm{mm}^{-1}$ & 0.078 \\
\hline $\mathrm{F}(000)$ & 1200.0 \\
\hline Crystal size $/ \mathrm{mm}^{3}$ & $0.2 \times 0.15 \times 0.15$ \\
\hline Radiation & $\operatorname{MoK} \alpha(\lambda=0.71073)$ \\
\hline \multicolumn{2}{|c|}{$2 \Theta$ range for data collection $/{ }^{\circ} 5.232$ to 55.146} \\
\hline Index ranges & $-14 \leq \mathrm{h} \leq 13,-20 \leq \mathrm{k} \leq 19,-22 \leq 1 \leq 21$ \\
\hline Reflections collected & 37271 \\
\hline Independent reflections & $3453\left[\mathrm{R}_{\mathrm{int}}=0.0598, \mathrm{R}_{\text {sigma }}=0.0397\right]$ \\
\hline Data/restraints/parameters & $3453 / 0 / 191$ \\
\hline Goodness-of-fit on $\mathrm{F}^{2}$ & 1.068 \\
\hline Final $\mathrm{R}$ indexes $[\mathrm{I}>=2 \sigma(\mathrm{I})]$ & $\mathrm{R}_{1}=0.0468, \mathrm{wR}_{2}=0.0915$ \\
\hline Final $\mathrm{R}$ indexes [all data] & $\mathrm{R}_{1}=0.0881, \mathrm{wR}_{2}=0.1070$ \\
\hline \multicolumn{2}{|c|}{ Largest diff. peak/hole / e $\AA^{-3} 0.22 /-0.21$} \\
\hline $\mathrm{CCDC}$ & 1868745 \\
\hline
\end{tabular}




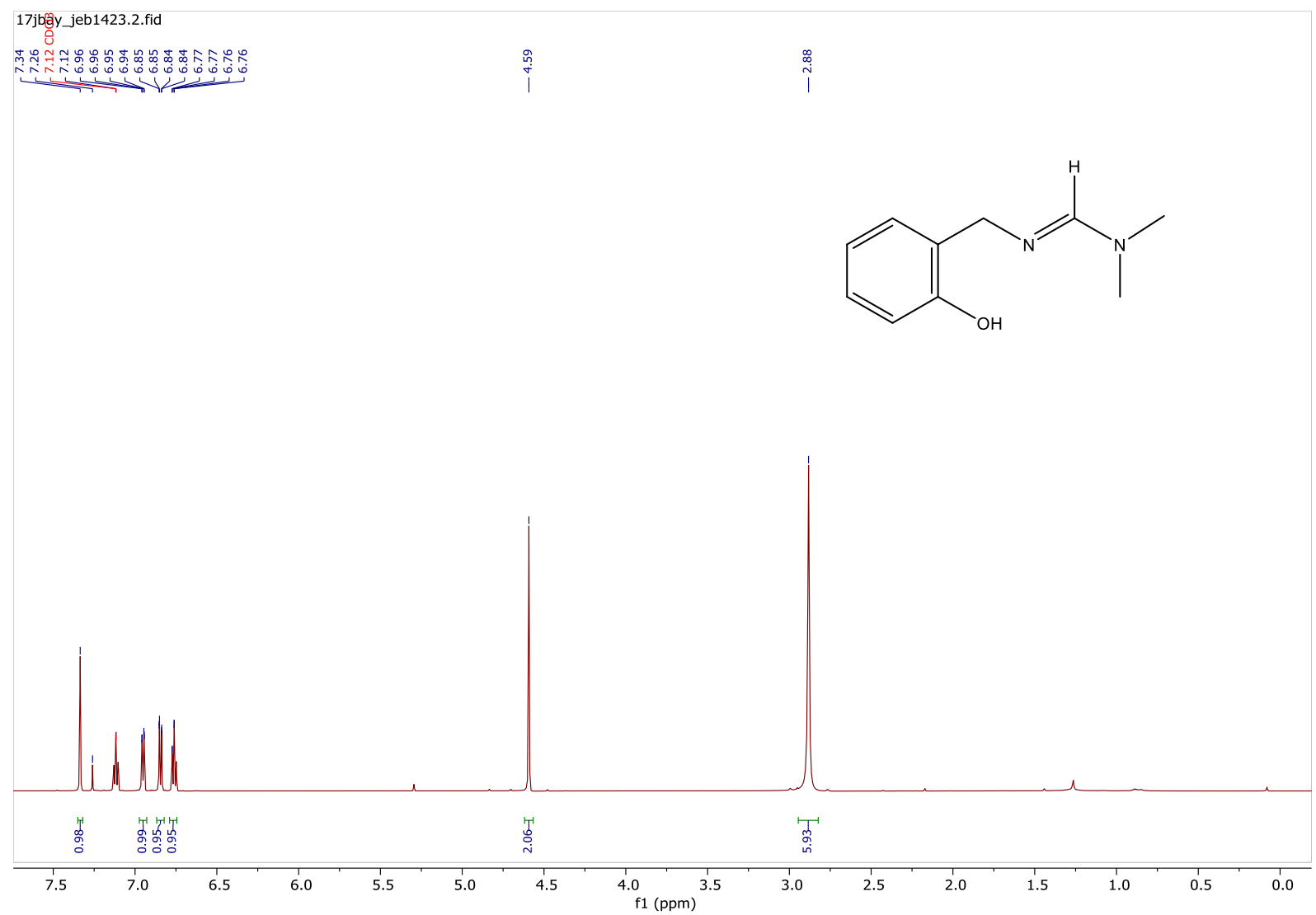

Figure S35: ${ }^{1} \mathrm{H}$ NMR (600 MHz, $\mathrm{CDCl}_{3}$ ) of $(E)-N$ '-(2-hydroxybenzyl)- $N, N$-dimethylformamidine $\mathbf{L 3 H}$

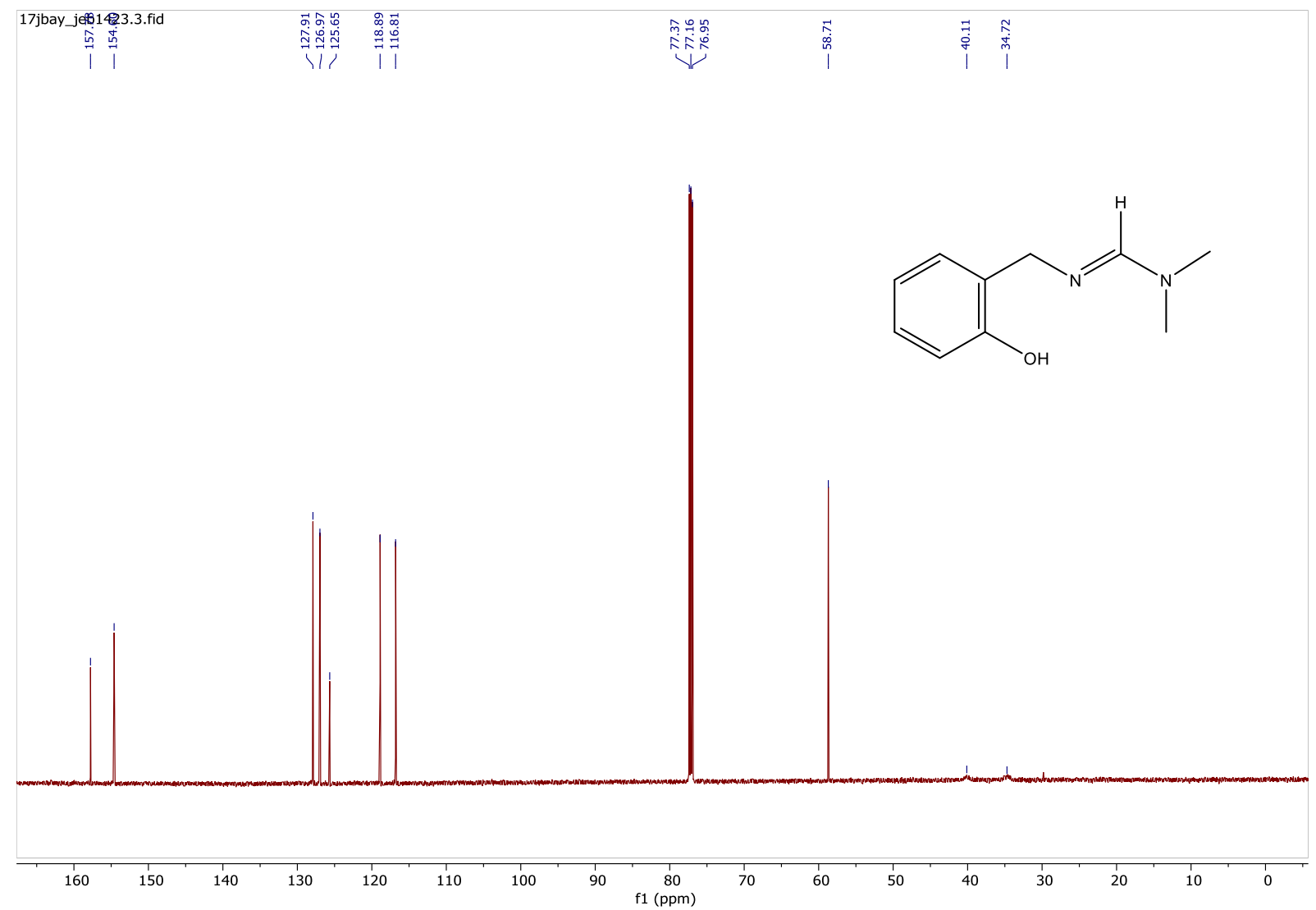

Figure S36: ${ }^{13} \mathrm{C}\left\{{ }^{1} \mathrm{H}\right\}$ NMR (151 MHz, $\left.\mathrm{CDCl}_{3}\right)$ of $(E)-N$ '-(2-hydroxybenzyl)- $N, N$-dimethylformamidine $\mathbf{L 3 H}$ 


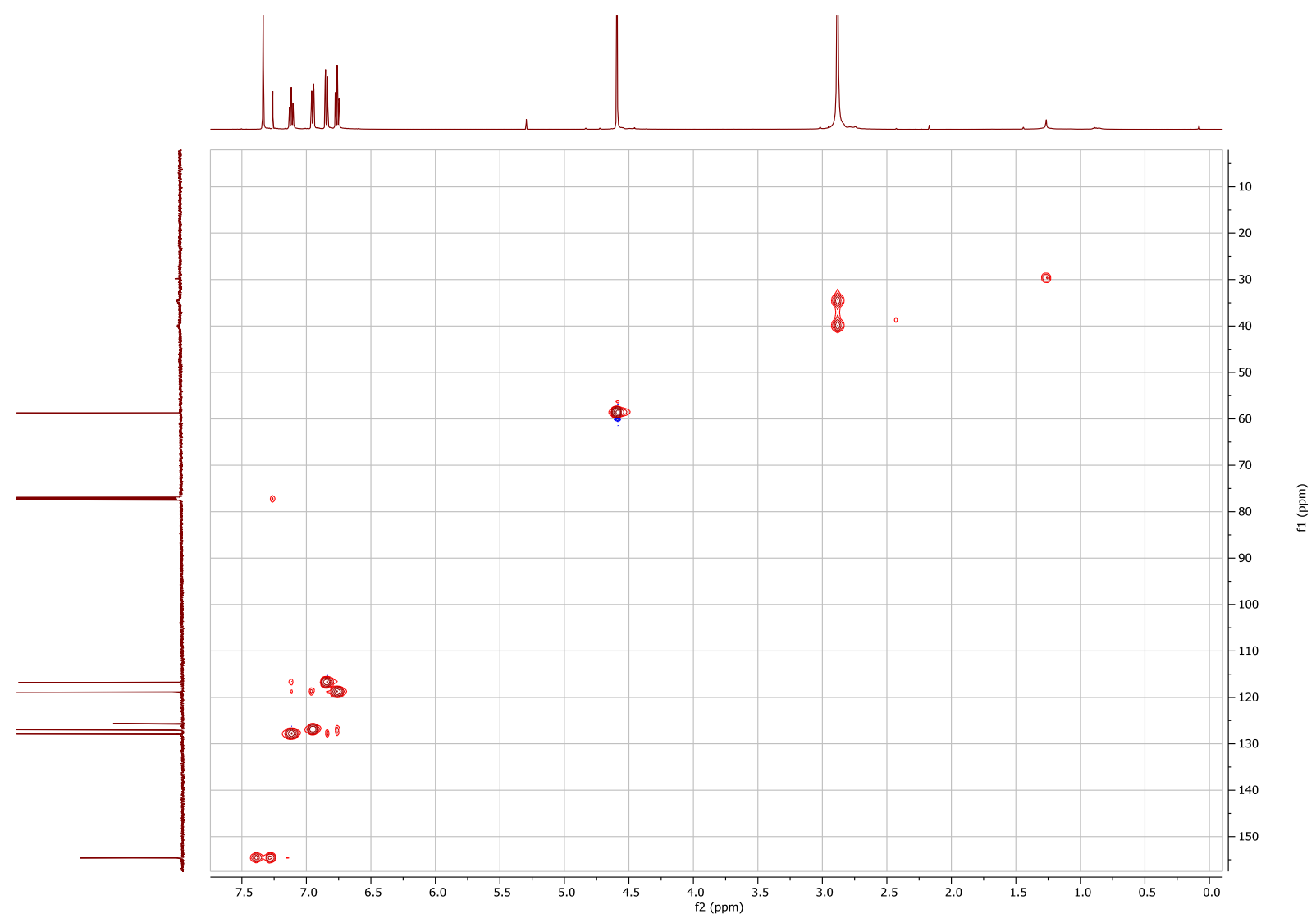

Figure S37: ${ }^{1} \mathrm{H}^{13} \mathrm{C} \mathrm{HSQC}\left(600 \mathrm{MHz} / 151 \mathrm{MHz}, \mathrm{CDCl}_{3}\right.$ ) of $(E)-N$ '-(2-hydroxybenzyl)- $N, N$-dimethylformamidine $\mathbf{L 3 H}$

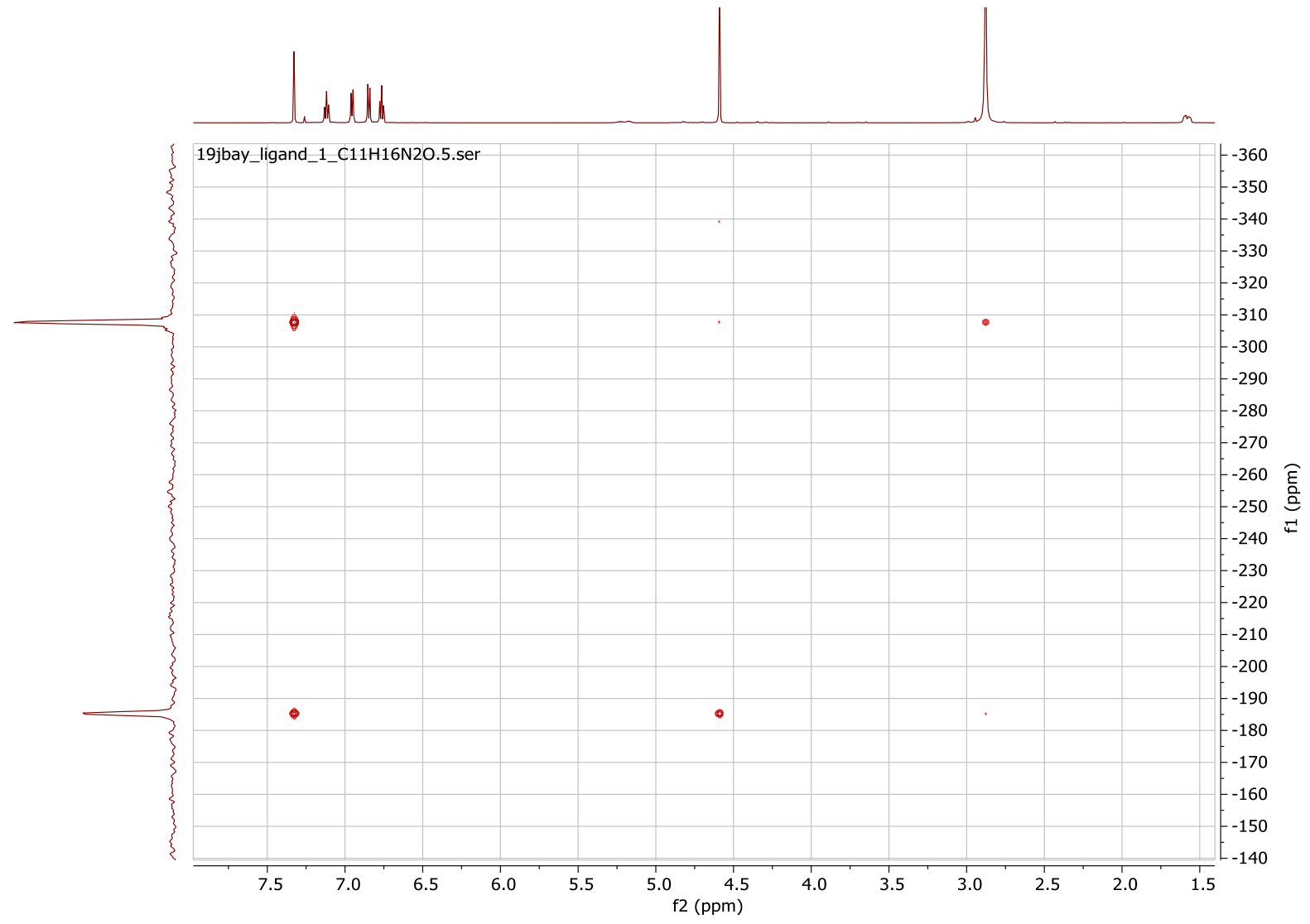

Figure S38: ${ }^{1} \mathrm{H}^{15} \mathrm{~N} \mathrm{HMBC}\left(600.23 \mathrm{MHz} / 43.3 \mathrm{MHz}, \mathrm{CDCl}_{3}\right)$ of $(E)-N$ '-(2-hydroxybenzyl)- $N, N$-dimethylformamidine $\mathbf{L 3 H}$ 


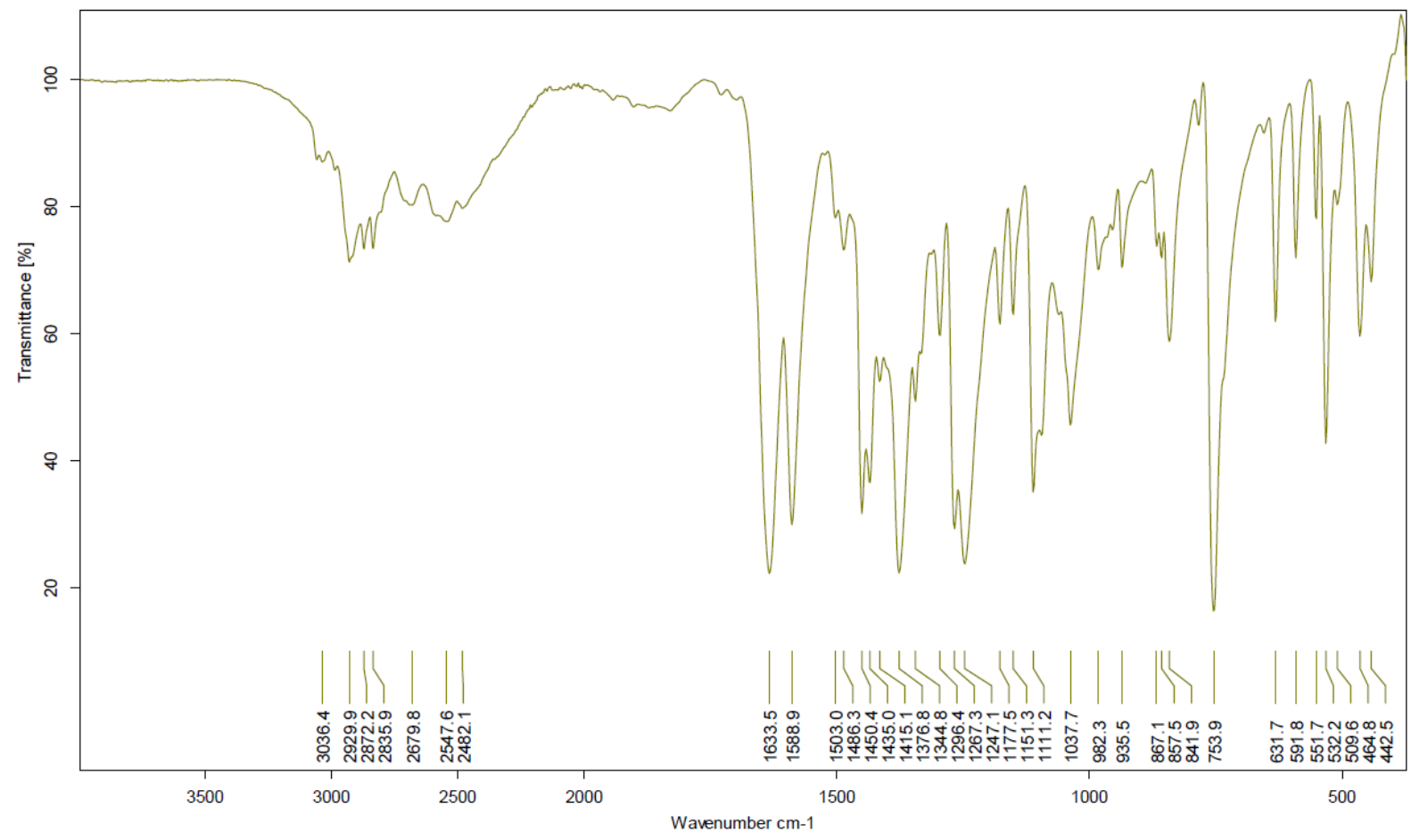

Figure S39: ATR-FT-IR spectrum of (E)- $N^{\prime}$-(2-hydroxybenzyl)- $N, N$-dimethylformamidine L3H

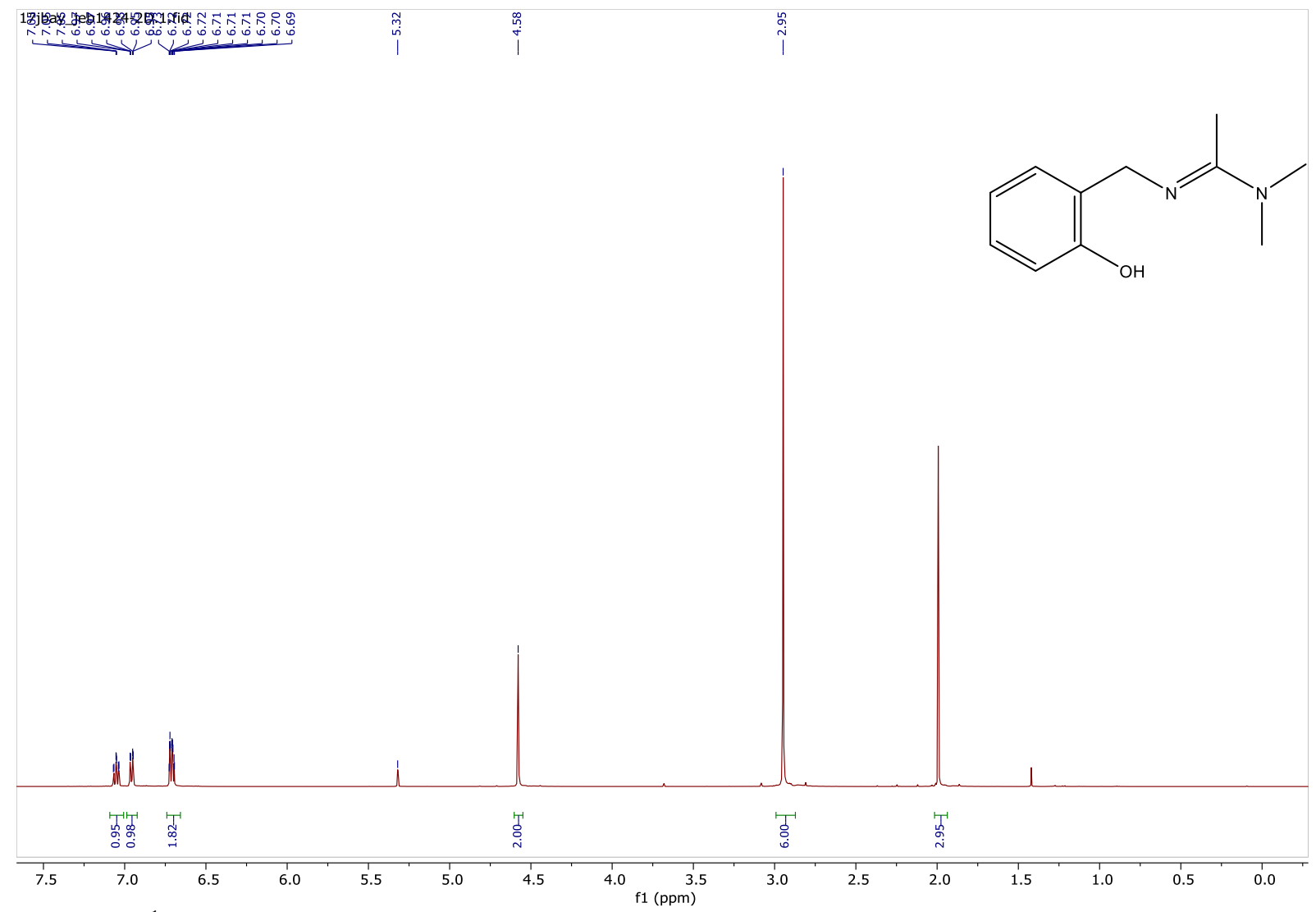

Figure S40: ${ }^{1} \mathrm{H}$ NMR $\left(500 \mathrm{MHz}, \mathrm{CD}_{2} \mathrm{Cl}_{2}\right)$ of $(E)-N$ '-(2-hydroxybenzyl)- $N, N$-dimethylacetamidine $\mathbf{L 4 H}$ 


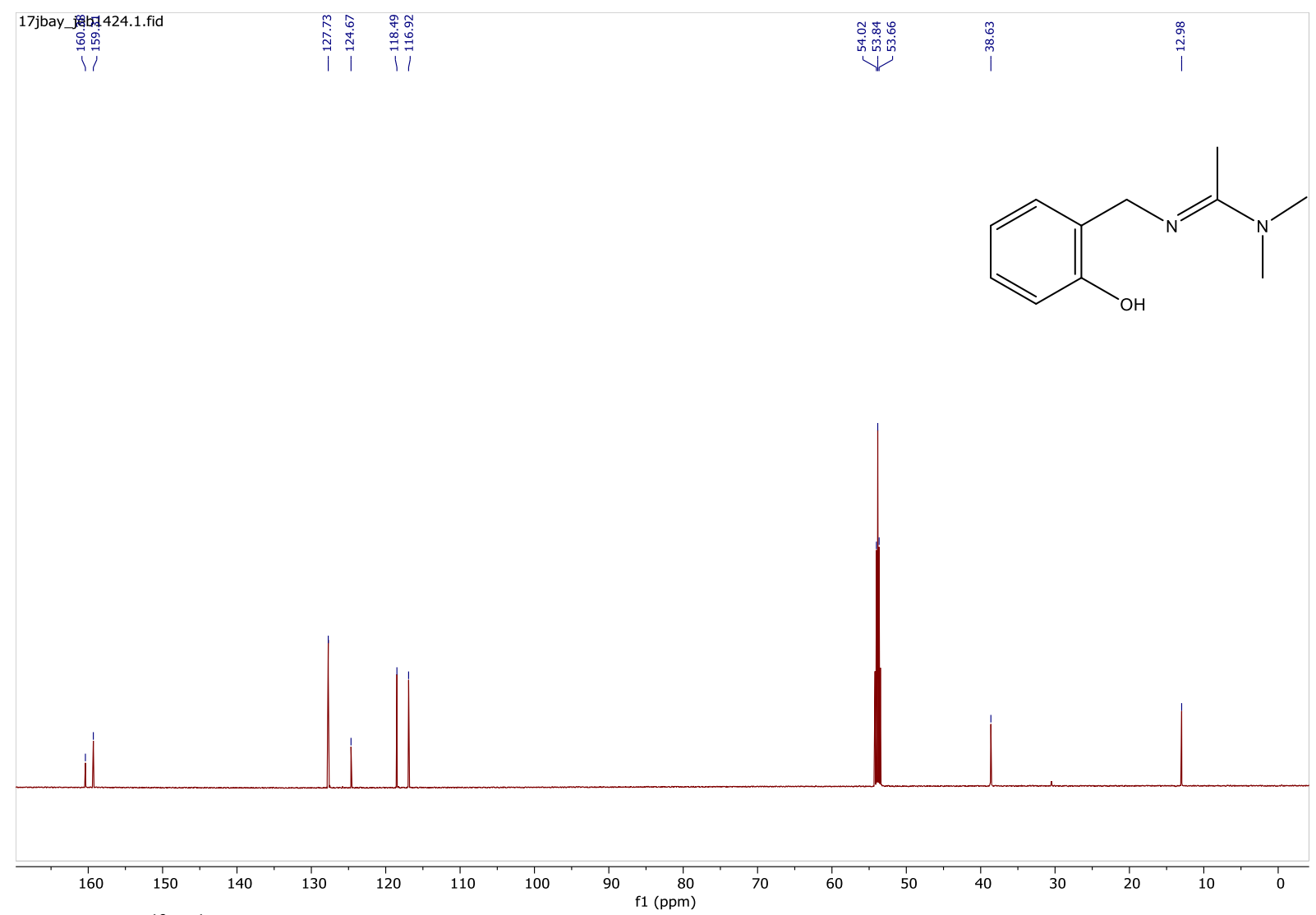

Figure S41: ${ }^{13} \mathrm{C}\left\{{ }^{1} \mathrm{H}\right\}$ NMR (151 MHz, $\mathrm{CD}_{2} \mathrm{Cl}_{2}$ ) of $(E)-N$ '-(2-hydroxybenzyl)- $N, N$-dimethylacetamidine $\mathbf{L 4 H}$

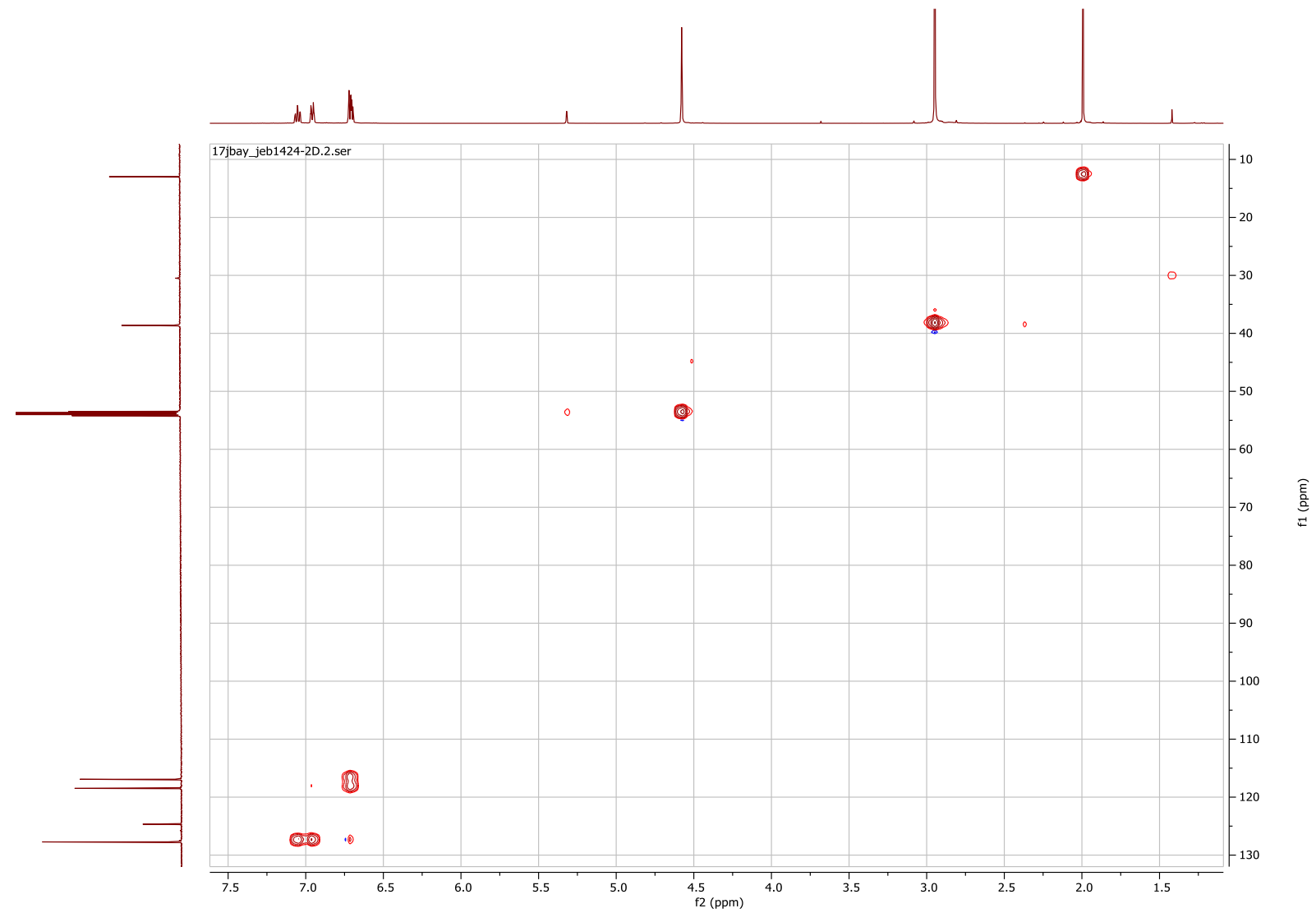

Figure S42: ${ }^{1} \mathrm{H}{ }^{13} \mathrm{C}$ HSQC $\left(500 \mathrm{MHz} / 151 \mathrm{MHz}, \mathrm{CD}_{2} \mathrm{Cl}_{2}\right.$ ) of $(E)-N$ '-(2-hydroxybenzyl)- $N, N$-dimethylacetamidine $\mathbf{L} \mathbf{4 H}$ 


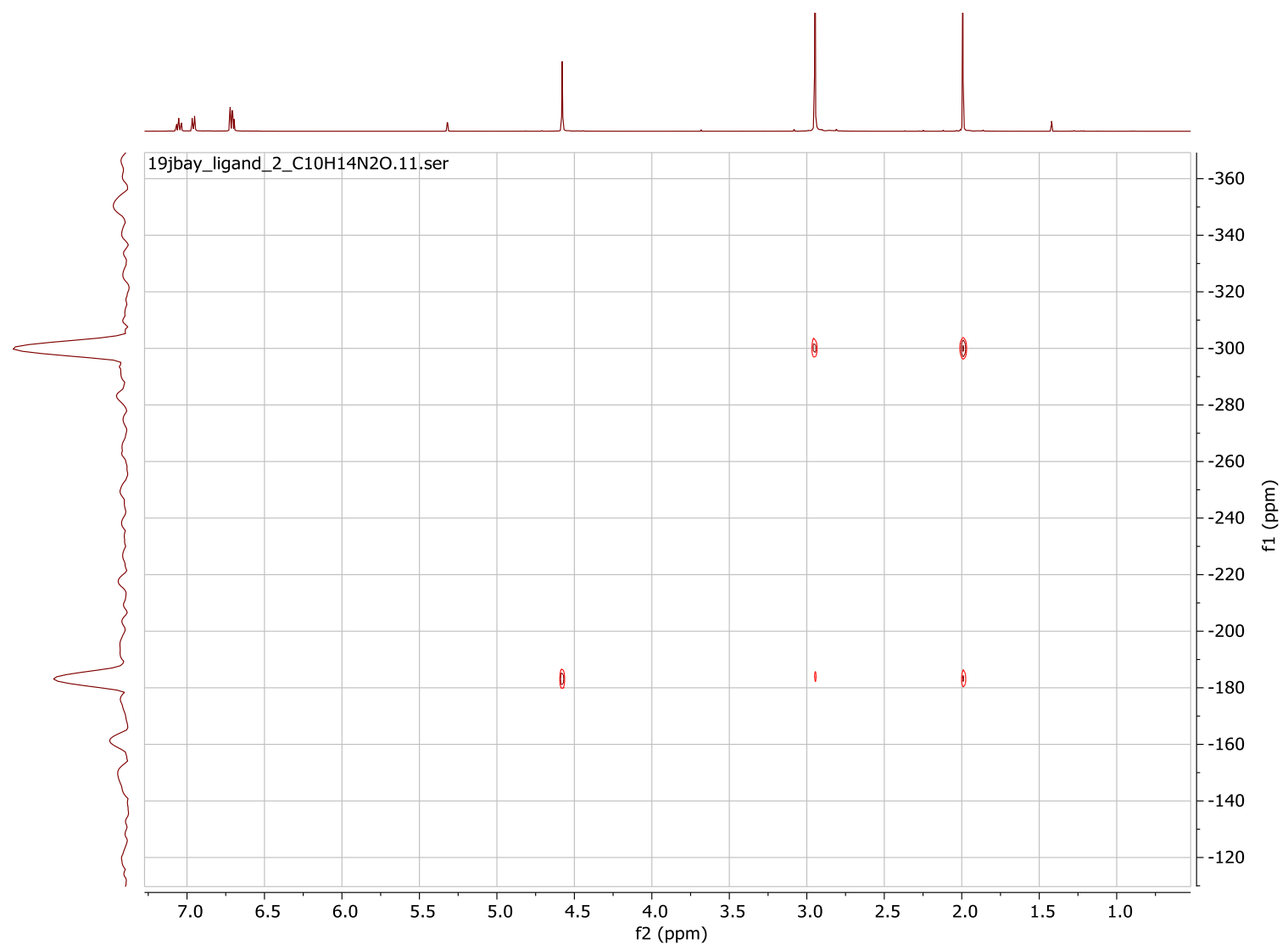

Figure S43: ${ }^{1} \mathrm{H}^{15} \mathrm{~N}$ HMBC (600.23 MHz / 43.3 MHz, $\mathrm{CD}_{2} \mathrm{Cl}_{2}$ ) of $(E)-N^{\prime}$-(2-hydroxybenzyl)- $N, N$-dimethylacetamidine $\mathbf{L 4 H}$

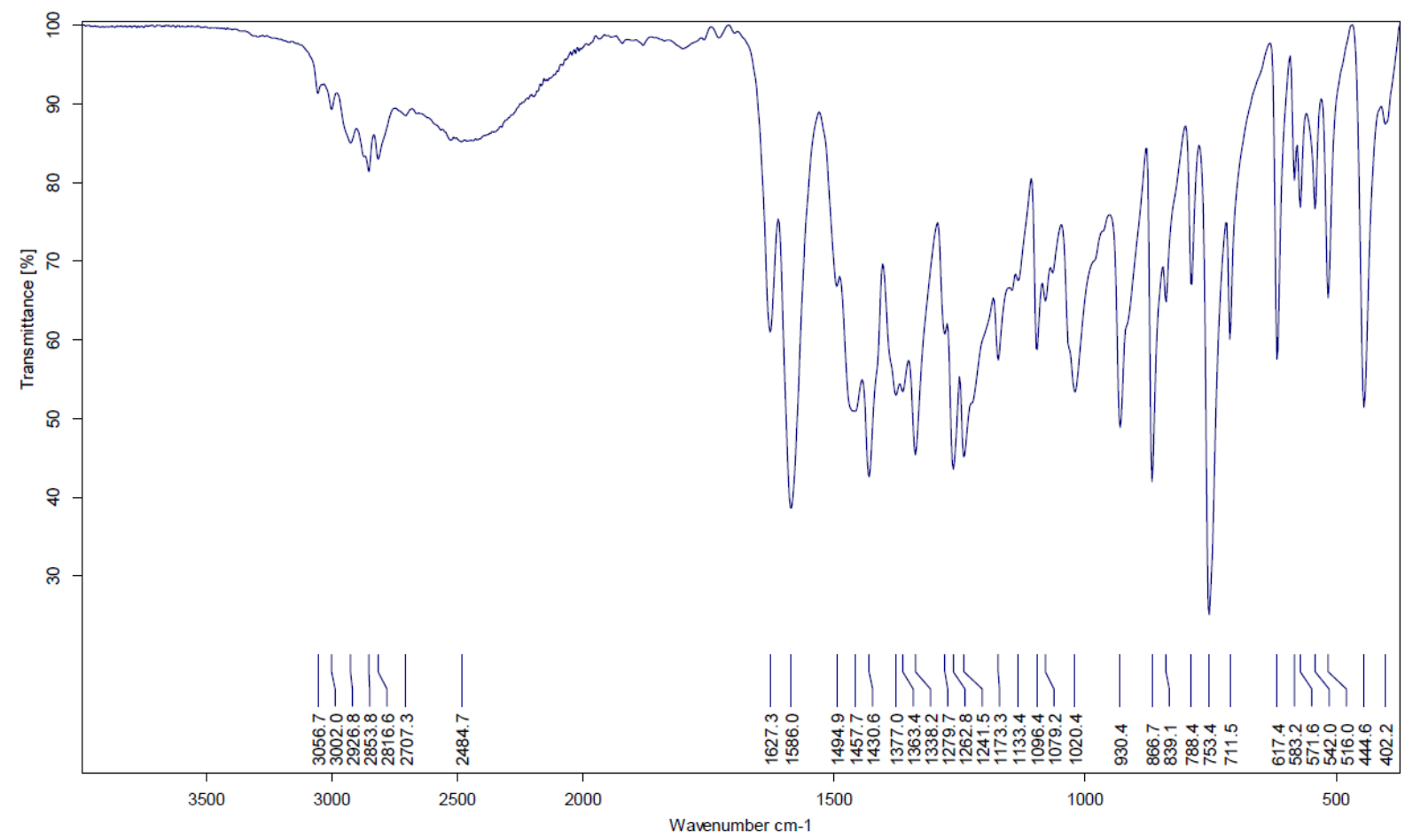

Figure S44: ATR-FT-IR spectrum of (E)- $N$ '-(2-hydroxybenzyl)- $N, N$-dimethylacetamidine L4H 


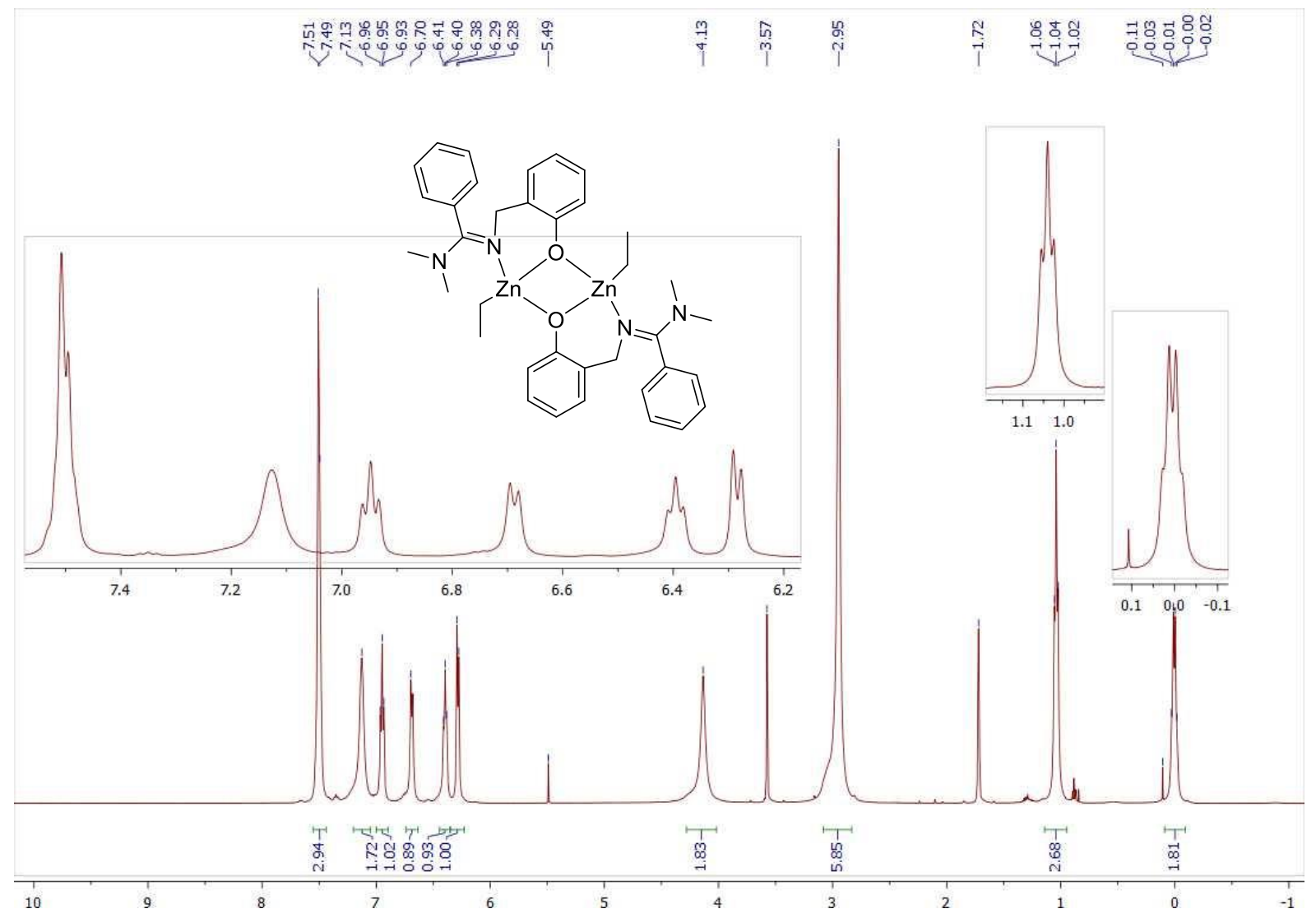

Figure S45: ${ }^{1} \mathrm{H}$ NMR $(500 \mathrm{MHz}, \mathrm{THF}-\mathrm{d} 8)$ of 1a

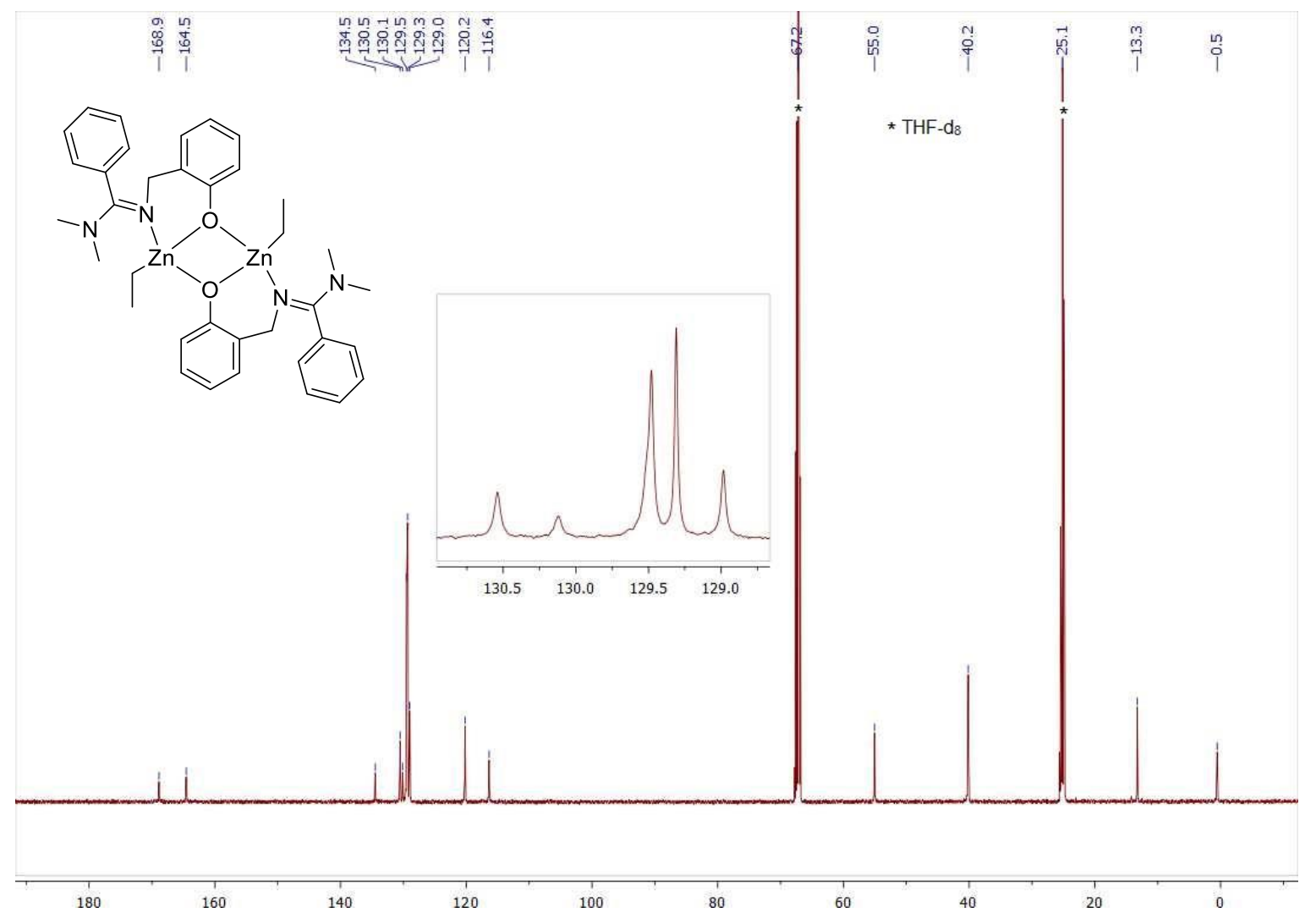

Figure S46: ${ }^{13} \mathrm{C}\left\{{ }^{1} \mathrm{H}\right\}$ NMR $\left(126 \mathrm{MHz}, \mathrm{THF}-\mathrm{d}_{8}\right)$ of $\mathbf{1 a}$ 


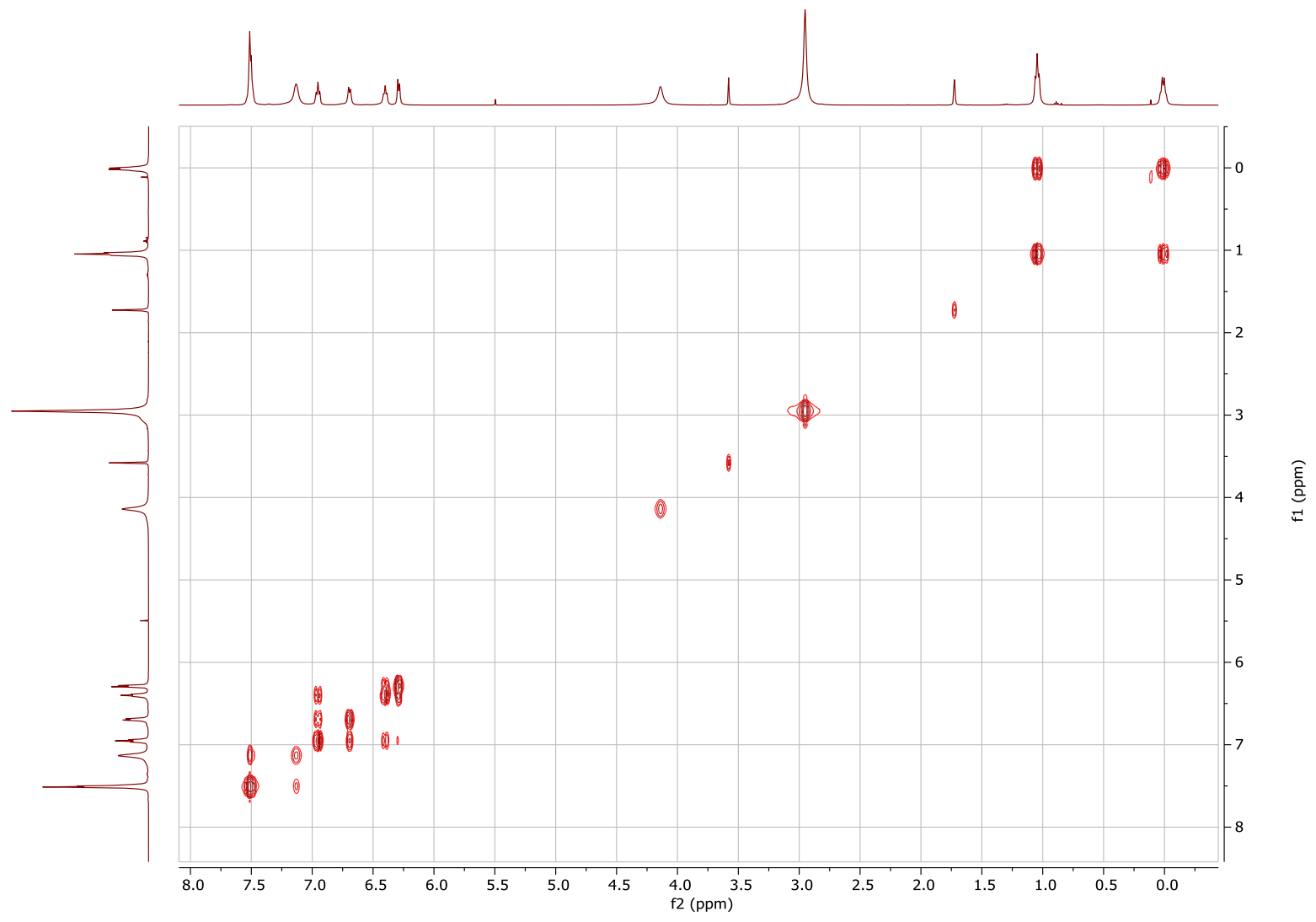

Figure S47: ${ }^{1} \mathrm{H}^{1} \mathrm{H}$ COSY (500 MHz, THF-d 8 ) of 1a

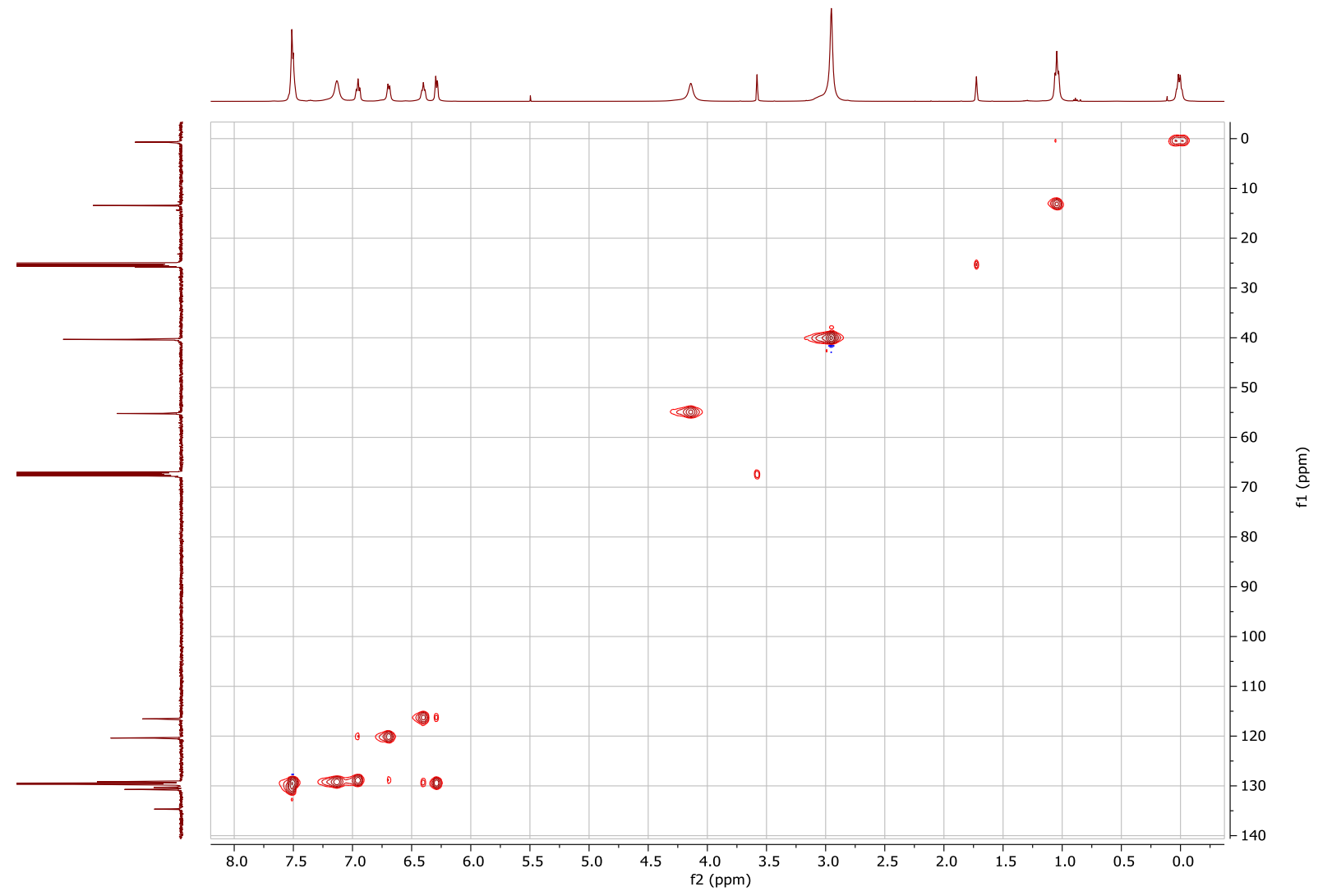

Figure S48: ${ }^{1} \mathrm{H}^{13} \mathrm{C}$ HSQC (500 MHz / $\left.126 \mathrm{MHz}, \mathrm{THF}-\mathrm{d} 8\right)$ of $\mathbf{1 a}$ 


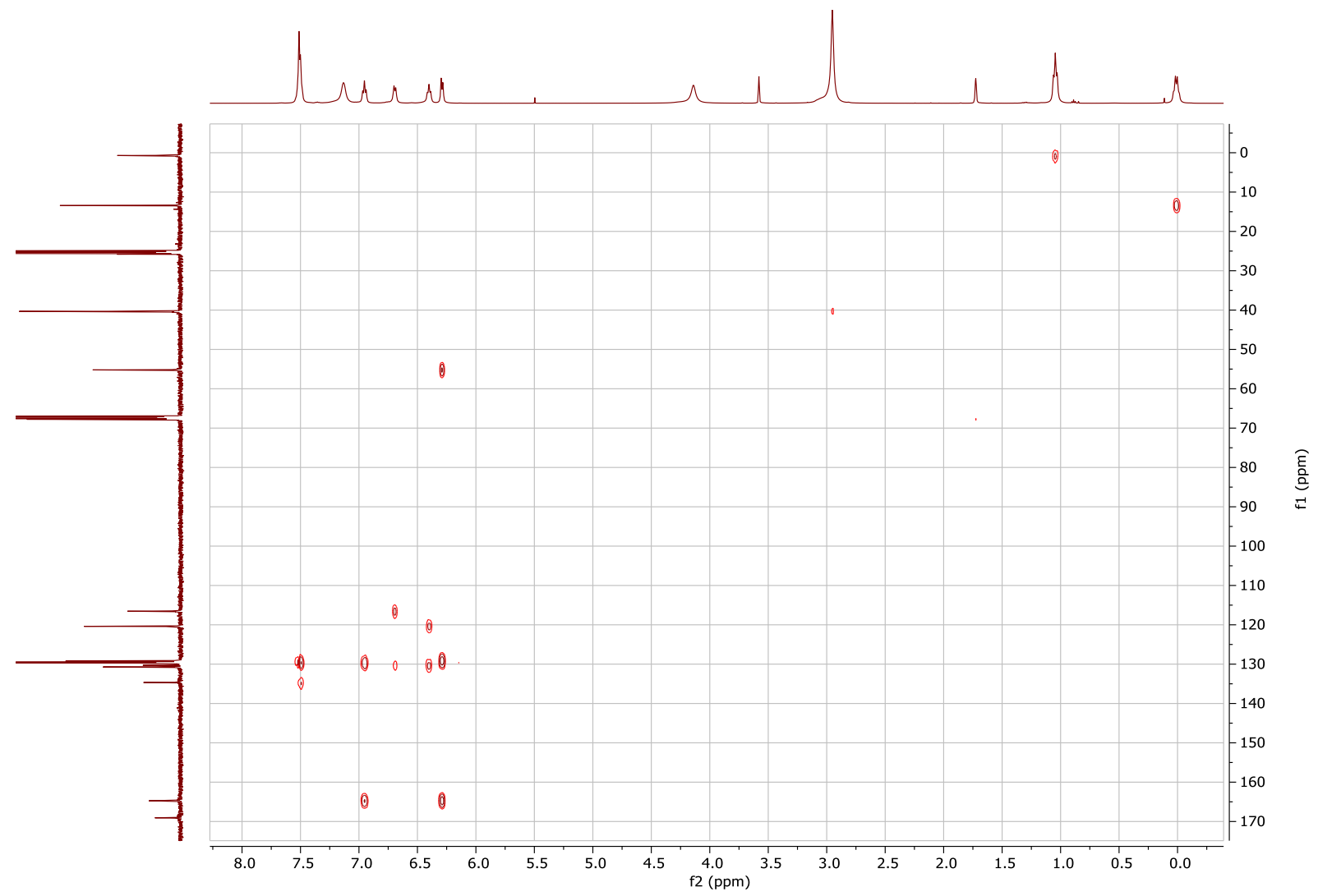

Figure S49: ${ }^{1} \mathrm{H}^{13} \mathrm{C}$ HMBC $(500 \mathrm{MHz} / 126 \mathrm{MHz}, \mathrm{THF}-\mathrm{d} 8)$ of $\mathbf{1 a}$

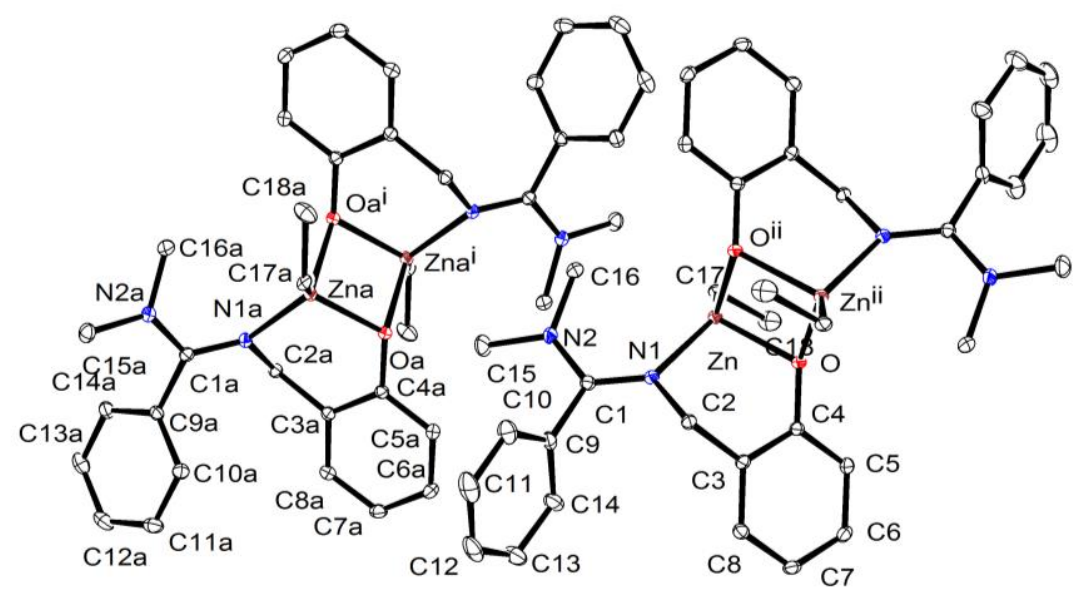

Figure S50: Crystal structure of 1a

Table S3: Crystal data and structure refinement of 1a

Identification code in cif

Empirical formula

Formula weight

Temperature/K

Crystal system

Space group

\section{C1a}

$\mathrm{C}_{36} \mathrm{H}_{44} \mathrm{~N}_{4} \mathrm{O}_{2} \mathrm{Zn}_{2}$

695.49

120

triclinic

P-1 


$\begin{array}{ll}\mathrm{a} / \AA & 10.1189(6) \\ \mathrm{b} / \AA & 11.3576(7) \\ \mathrm{c} / \AA & 15.5735(10) \\ \alpha /{ }^{\circ} & 87.198(2) \\ \beta /{ }^{\circ} & 79.883(2) \\ /^{\circ} & 73.090(2) \\ \mathrm{V}^{\circ} & 1685.79(18) \\ \mathrm{Z} & 2 \\ \rho_{\text {calcg }} / \mathrm{cm}^{3} & 1.370 \\ \mu / \mathrm{mm}^{-1} & 1.460 \\ \mathrm{~F}(000) & 728.0 \\ \mathrm{Crystal} \text { size/mm } & \\ \text { Radiation } & 0.5 \times 0.5 \times 0.5 \\ 2 \Theta \text { range for data collection/ } & { }^{3} 5.396 \text { to } 55.104 \\ \text { Index ranges } & -13 \leq \mathrm{h} \leq 13,-14 \leq \mathrm{k} \leq 14,-20 \leq 1 \leq 20 \\ \text { Reflections collected } & 48578 \\ \text { Independent reflections } & 7724\left[\mathrm{R}_{\text {int }}=0.0199, \mathrm{R}_{\text {sigma }}=0.0127\right] \\ \text { Data/restraints/parameters } & 7724 / 6 / 403 \\ \text { Goodness-of-fit on } \mathrm{F}^{2} & 1.059 \\ \text { Final } \mathrm{R} \text { indexes }[\mathrm{I}>=2 \sigma(\mathrm{I})] & \mathrm{R}_{1}=0.0245, \mathrm{wR}_{2}=0.0627 \\ \text { Final R indexes [all data] } & \mathrm{R}_{1}=0.0275, \mathrm{wR}_{2}=0.0649 \\ \text { Largest diff. peak/hole / e } \AA^{-3} 0.78 /-0.33 \\ \text { CCDC } & 1868746\end{array}$

\section{DOSY analyses:}

All spectra were recorded on an Avance III HD $600 \mathrm{MHz}$ Bruker spectrometer equipped with a $5 \mathrm{~mm}$ BBOF probe (with gradient strength $5.099 \mathrm{G} / \mathrm{cmA}$ ), and using $2.5 \mathrm{~mm}$ NMR tubes at 298K. All samples were prepared as follow: $30 \mathrm{mmol}$ of the analyte were dissolved in $990 \mu \mathrm{L}$ of $\mathrm{CD}_{2} \mathrm{Cl}_{2}$ and $10 \mu \mathrm{L}$ of pentane used as internal reference. A test portion of $250 \mu \mathrm{L}$ was then introduced in a $2.5 \mathrm{~mm}$ NMR tube and finally introduced in the magnet. The specific parameters of each sample (d1, p1, ns, $\mathrm{rg}$ ) were optimized on a standard $1 \mathrm{D}$ acquisition $\left({ }^{1} \mathrm{H}\right.$ sequence zg30, 16 scans), and recalled in the dosy experiment (ledbpgp2s, 32 points, linear gradient from $95 \%$ to $5 \%$ ). The diffusion delay d20 was fixed at $0,01 \mathrm{~s}$, while the diffusion gradient length p30 was adapted to get a 95\% intensity decrease between the first spectrum and the last one. A calibration plot was determined using different compounds in a mass range of 72 to $623 \mathrm{~g} / \mathrm{mol}$.

DOSY data were processed using Topspin $3.5 \mathrm{pl} 7$ with the dosy $2 \mathrm{~d}$ exponential processing method. The results were formatted using a linear scale and a noise sensitivity factor of 20 . All diffusion coefficients were read on the two-dimensional spectrum obtained after processing. The diffusion coefficients obtained for the compounds used for the calibration are summarized in the following table:

Table S4: Diffusion coefficients obtained for the compounds used for the calibration and calibration curve

\begin{tabular}{c|cccc} 
Compound & $M W(\mathrm{~g} / \mathrm{mol})$ & $D\left(\mathrm{~m}^{2} / \mathrm{s}\right)$ & $\log M W$ & $\log D$ \\
\hline pentane & 72.2 & $3.30 \mathrm{E}-09$ & 1.8585372 & -8.4814861 \\
$\mathrm{NEt}_{3}$ & 101.2 & $2.60 \mathrm{E}-09$ & 2.00518051 & -8.5850267 \\
$\mathrm{~L} 3 \mathrm{H}$ & 178.1 & $2.00 \mathrm{E}-09$ & 2.25066392 & -8.69897 \\
$\mathrm{Ph}_{3} \mathrm{CH}$ & 244.3 & $1.72 \mathrm{E}-09$ & 2.38792347 & -8.7644716 \\
$\mathrm{PPh}_{3}$ & 262.3 & $1.77 \mathrm{E}-09$ & 2.41879829 & -8.7520267 \\
$\mathrm{Ph}_{2} \mathrm{CCPh}$ & 332.4 & $1.50 \mathrm{E}-09$ & 2.52166102 & -8.8239087 \\
$\mathrm{Binap}_{2}$ & 622.7 & $1.26 \mathrm{E}-09$ & 2.79427887 & -8.8996295
\end{tabular}

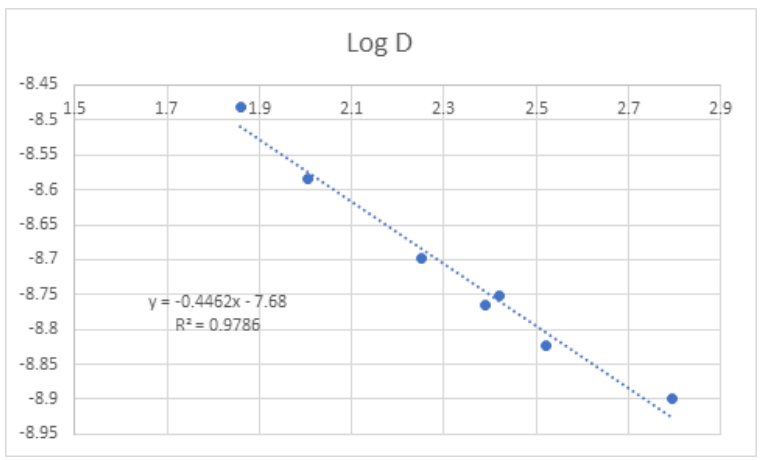




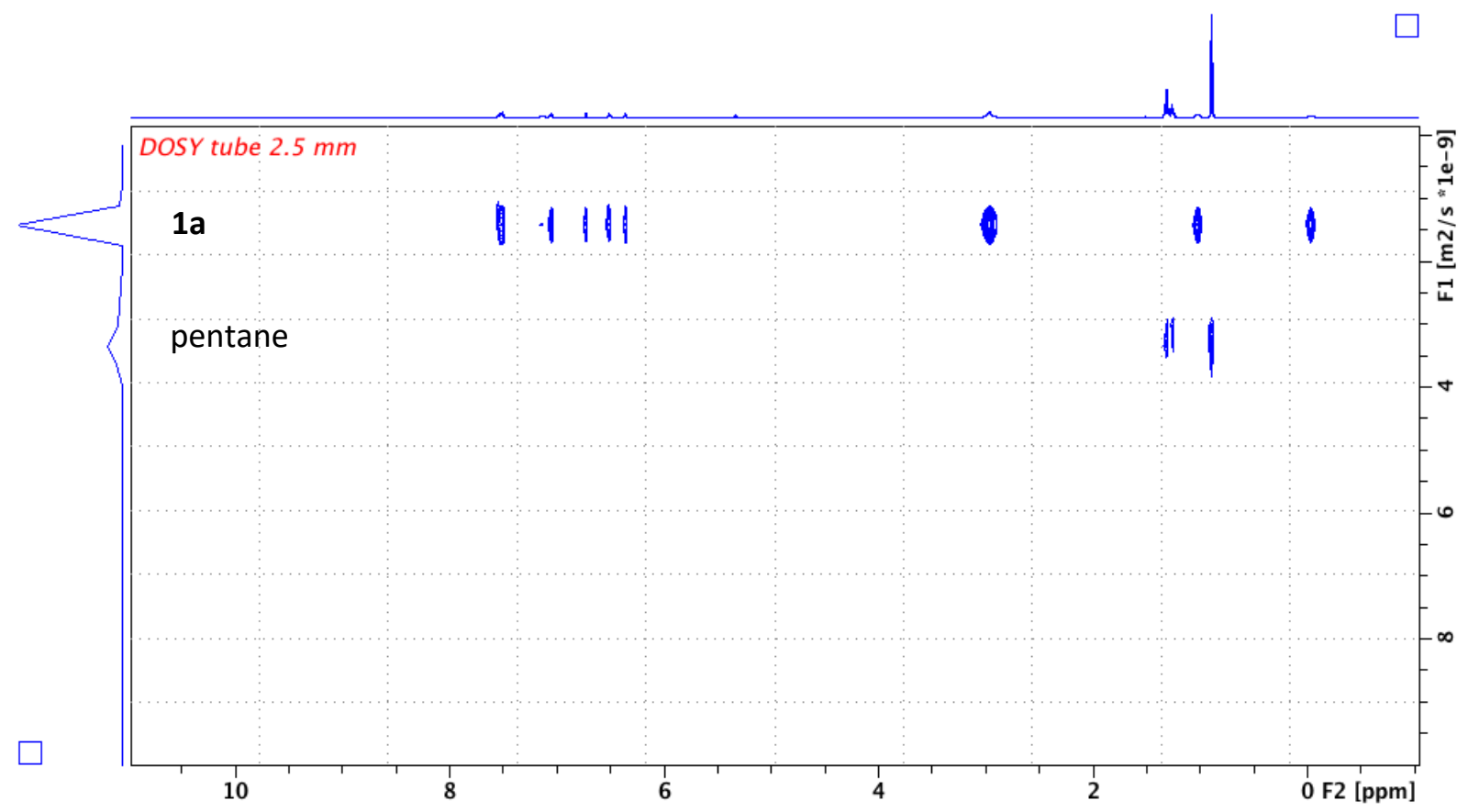

Figure S51: Processed DOSY spectrum of 1a

Table S5: Theoretical and experimental diffusion coefficients estimated for 1a (dimer and monomer)

\begin{tabular}{l|llll}
\multicolumn{1}{c}{$M W(\mathrm{~g} / \mathrm{mol})$} & Dth & $\log M W$ & $\log D$ \\
\hline 1a $\left(\mathrm{C}_{36} \mathrm{H}_{44} \mathrm{~N}_{4} \mathrm{O}_{2} \mathrm{Zn} \mathrm{n}_{2}\right)$ & 695,5 & $1,13 \mathrm{E}-09$ & 2,84229713 & $-8,948233$ \\
$\mathrm{C}_{18} \mathrm{H}_{22} \mathrm{~N}_{2} \mathrm{OZn}$ & 347,8 & $1,53 \mathrm{E}-09$ & 2,54132958 & $-8,8139413$ \\
& & Dexp & & \\
& 581,794406 & $1,22 \mathrm{E}-09$ & 2,76476954 & $-8,9136402$
\end{tabular}




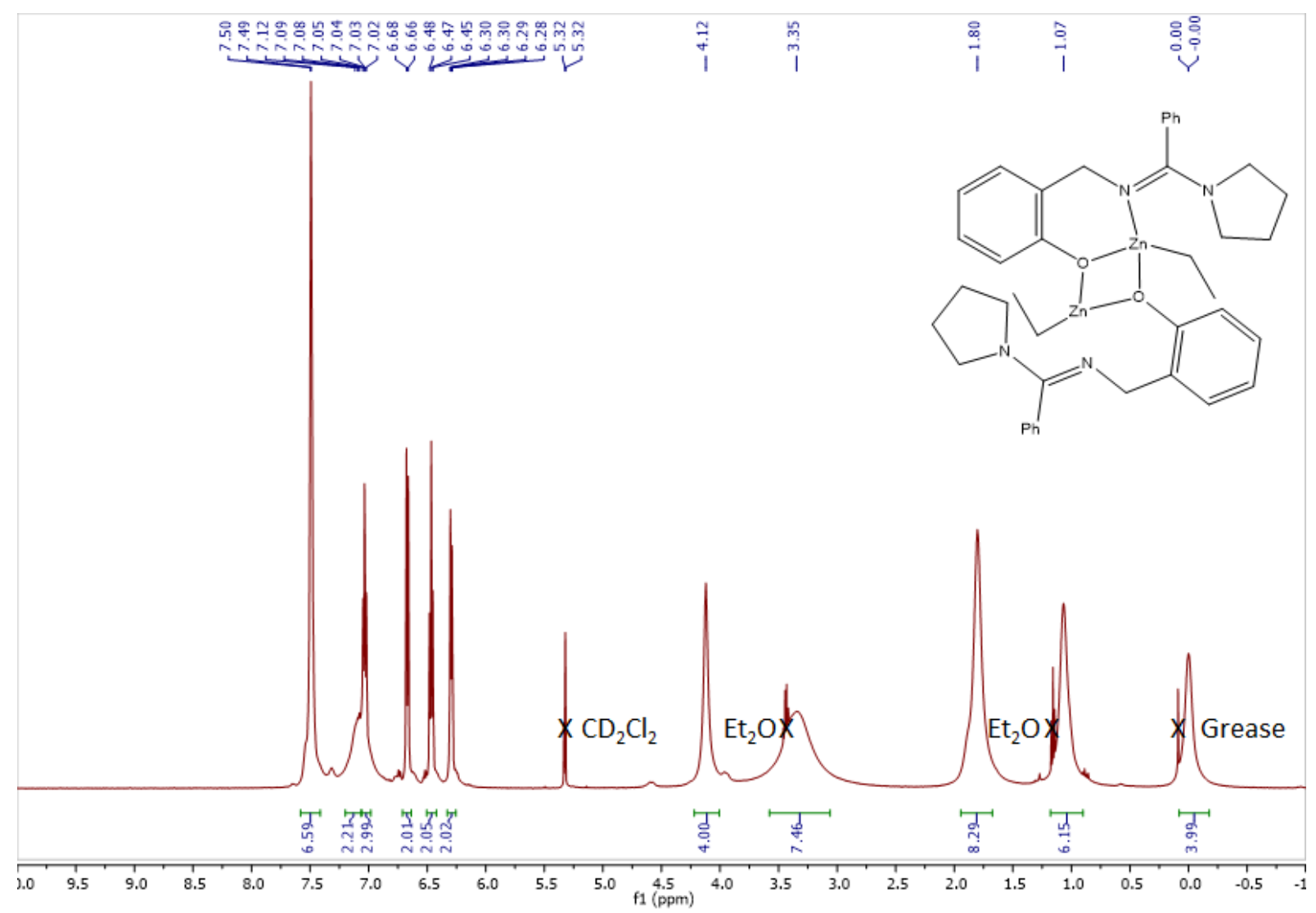

Figure S52: ${ }^{1} \mathrm{H}$ NMR $\left(500 \mathrm{MHz}, \mathrm{CD}_{2} \mathrm{Cl}_{2}\right)$ of $\mathbf{2 a}$

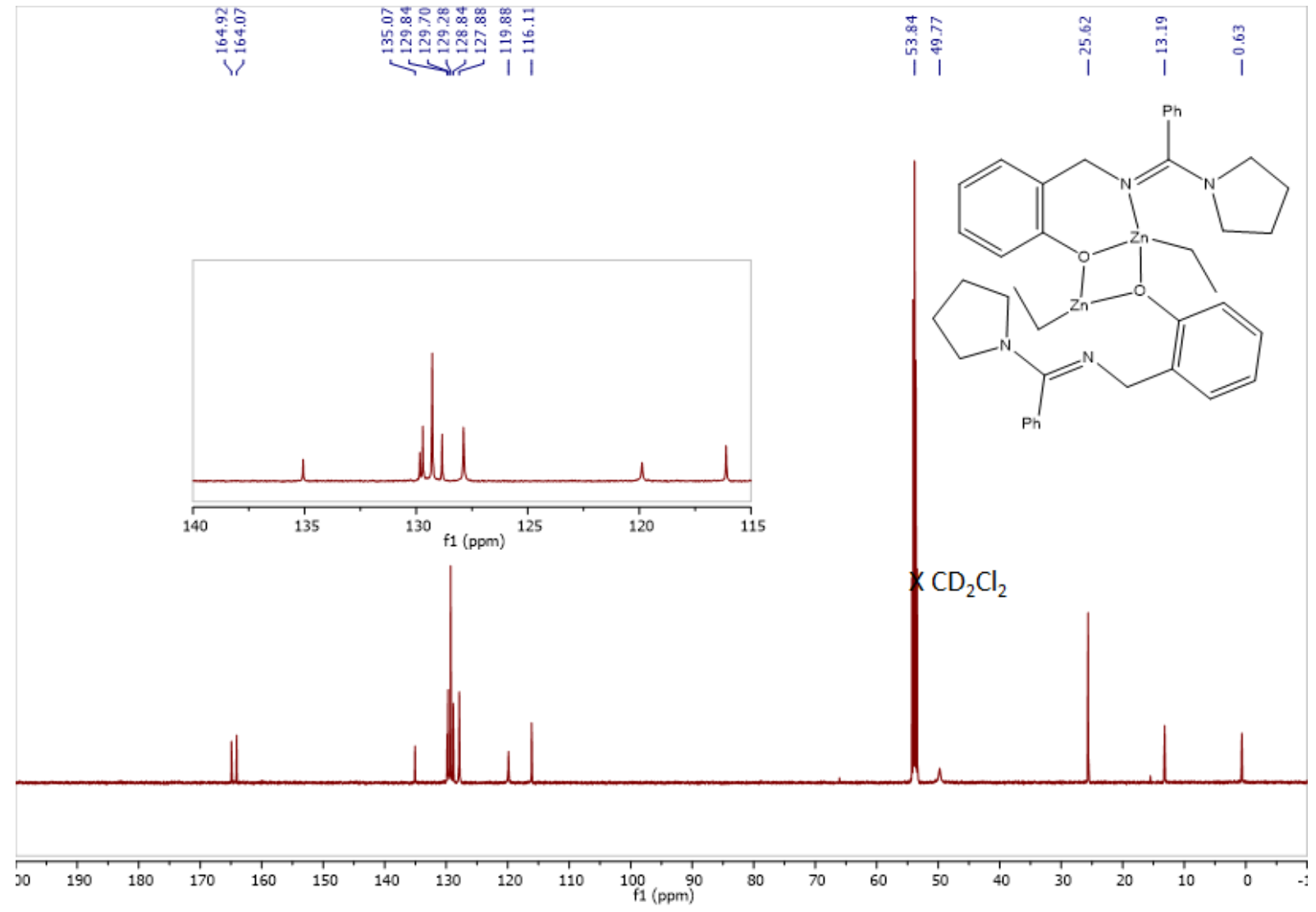

Figure S53: ${ }^{13} \mathrm{C}\left\{{ }^{1} \mathrm{H}\right\}$ NMR $\left(126 \mathrm{MHz}, \mathrm{CD}_{2} \mathrm{Cl}_{2}\right)$ of $\mathbf{2 a}$ 


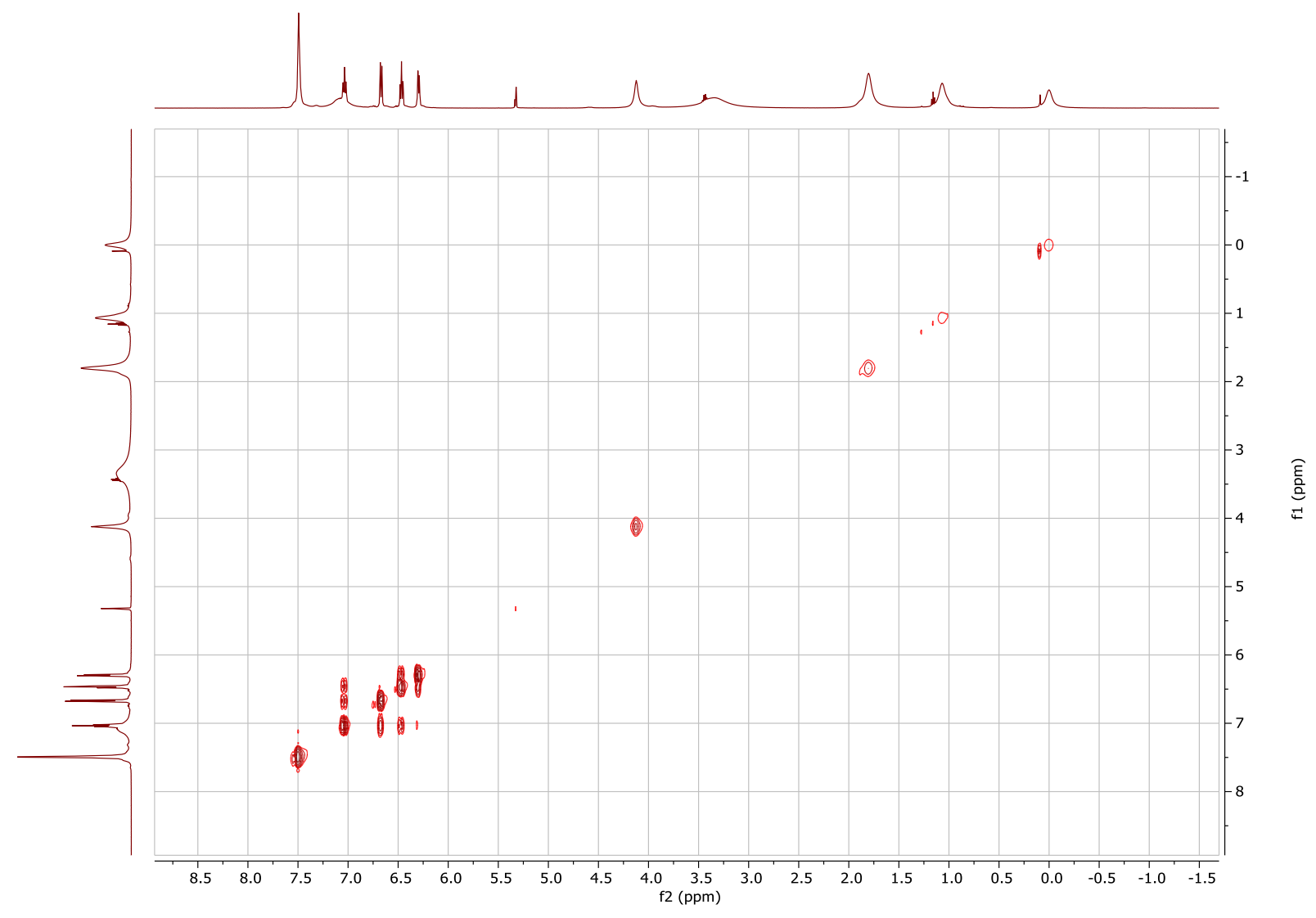

Figure S54: ${ }^{1} \mathrm{H}^{1} \mathrm{H} \operatorname{COSY}\left(500 \mathrm{MHz}, \mathrm{CD}_{2} \mathrm{Cl}_{2}\right)$ of $\mathbf{2 a}$

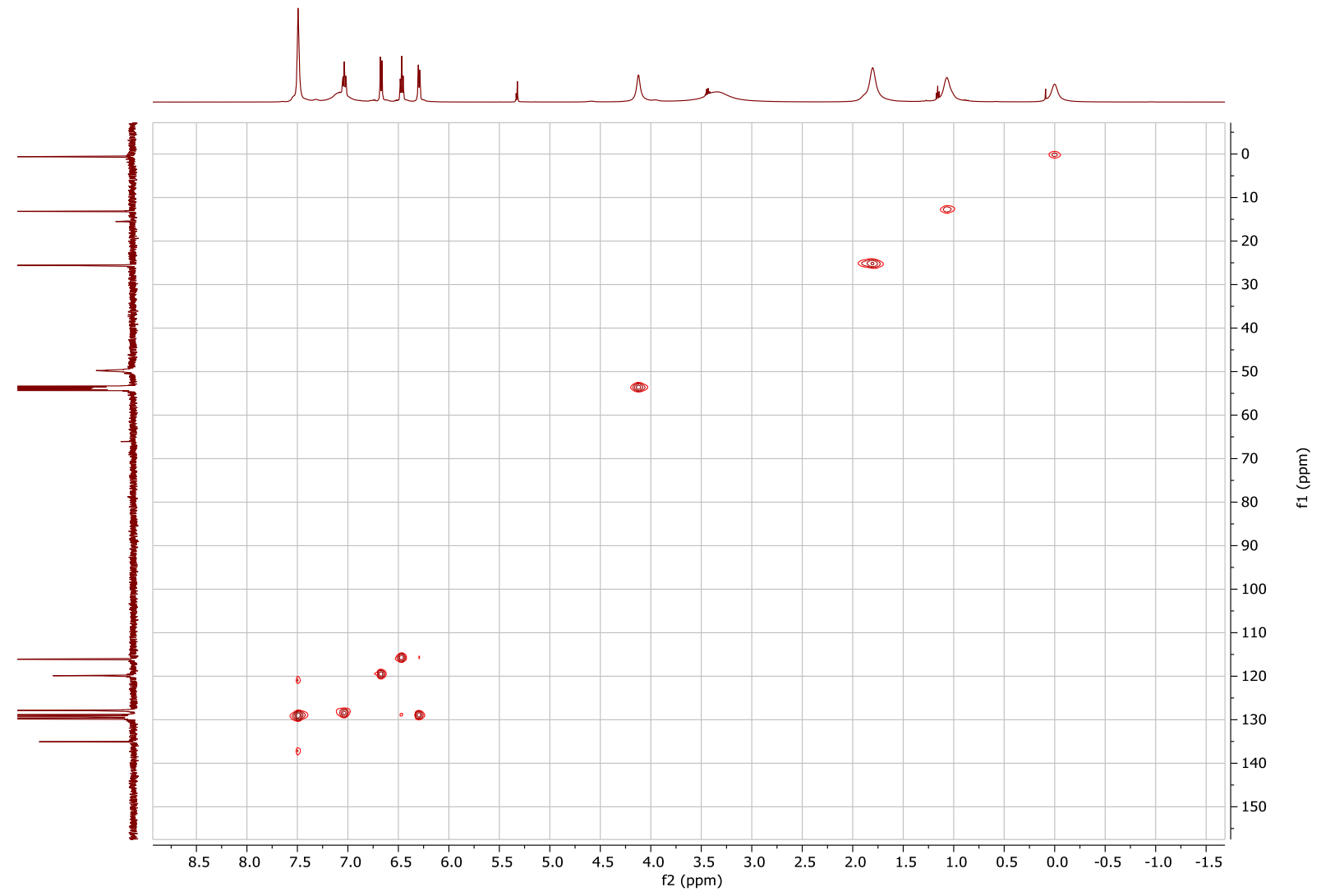

Figure S55: ${ }^{1} \mathrm{H}{ }^{13} \mathrm{C} \mathrm{HSQC}\left(500 \mathrm{MHz} / 126 \mathrm{MHz}, \mathrm{CD}_{2} \mathrm{Cl}_{2}\right)$ of $\mathbf{2 a}$ 


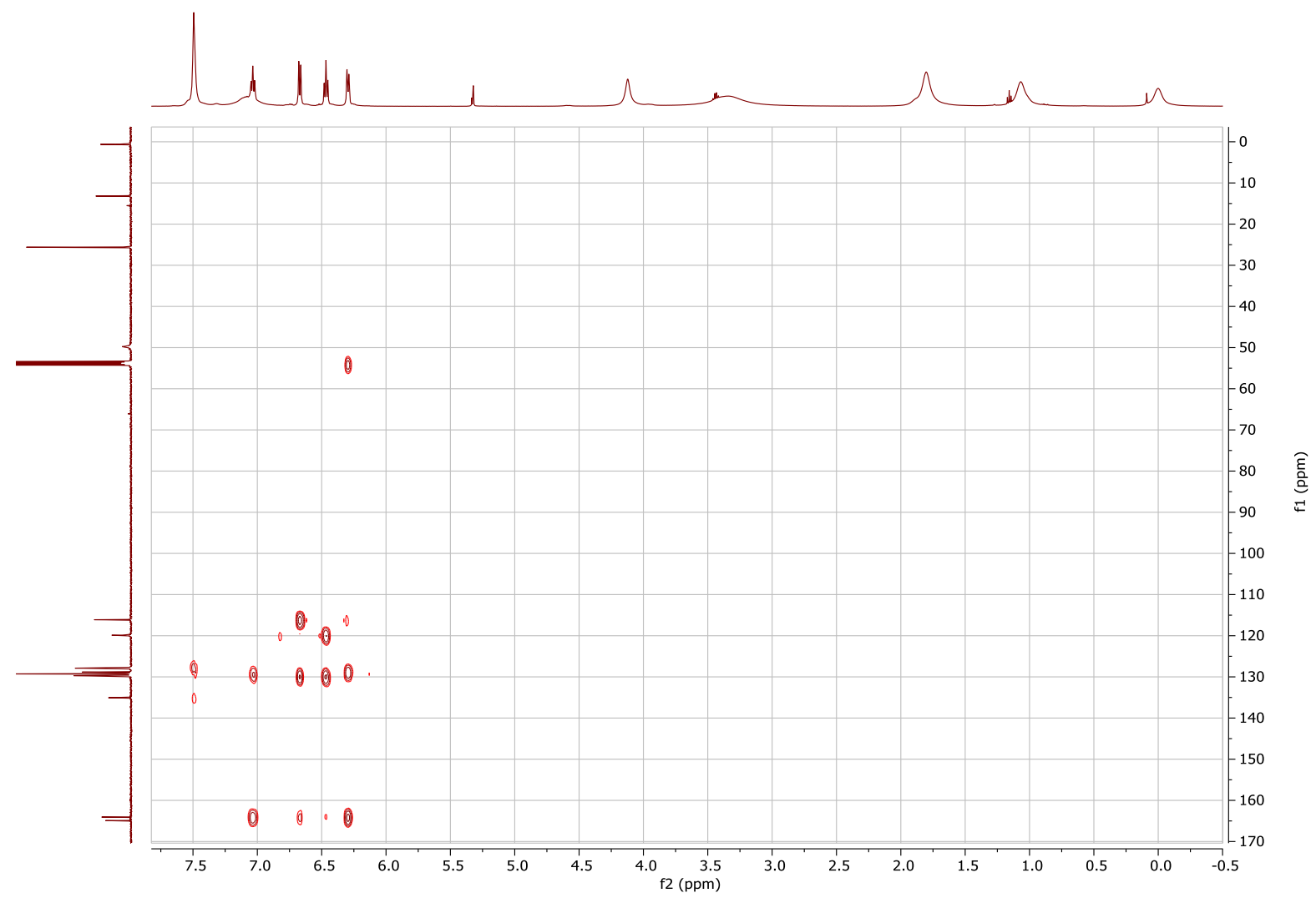

Figure S56: ${ }^{1} \mathrm{H}^{13} \mathrm{C} \mathrm{HMBC}\left(500 \mathrm{MHz} / 126 \mathrm{MHz}, \mathrm{CD}_{2} \mathrm{Cl}_{2}\right)$ of $\mathbf{2 a}$

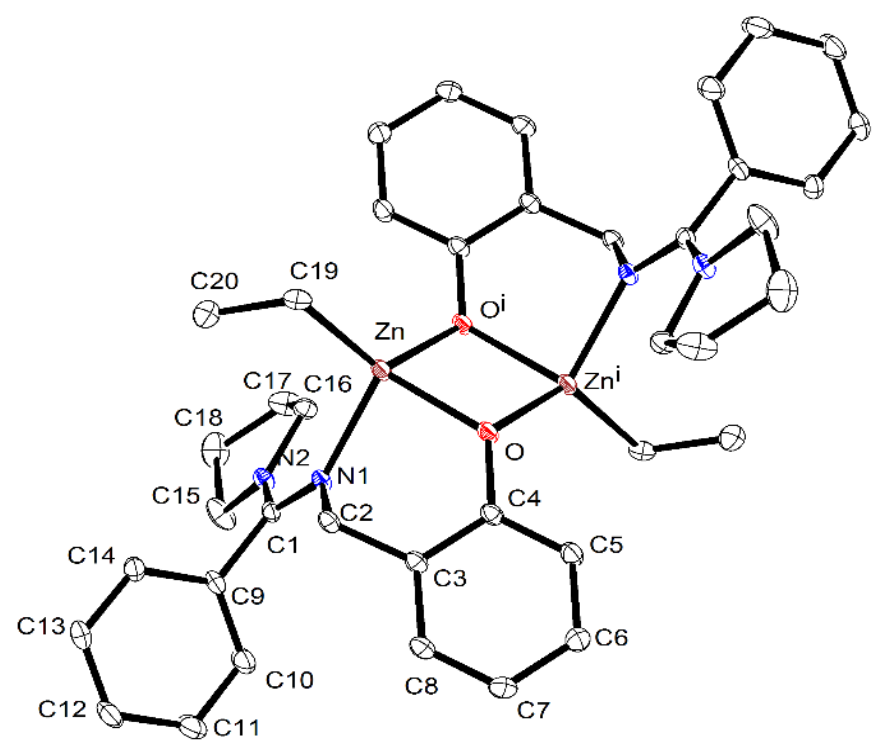

Figure S57: Crystal structure of 2a 
Table S6: Crystal data and structure refinement of $\mathbf{2 a}$

\begin{tabular}{|c|c|}
\hline Identification code in cif & $\mathbf{C 2 a}$ \\
\hline Empirical formula & $\mathrm{C}_{20} \mathrm{H}_{24} \mathrm{~N}_{2} \mathrm{OZn}$ \\
\hline Formula weight & 373.78 \\
\hline Temperature/K & 115.0 \\
\hline Crystal system & monoclinic \\
\hline Space group & $P 2{ }_{1} / c$ \\
\hline $\mathrm{a} / \AA ̊$ & $10.2386(5)$ \\
\hline $\mathrm{b} / \AA$ & $17.2646(8)$ \\
\hline $\mathrm{c} / \AA$ & $9.9335(5)$ \\
\hline$\alpha /^{\circ}$ & 90 \\
\hline$\beta /{ }^{\circ}$ & $96.097(2)$ \\
\hline$\gamma /{ }^{\circ}$ & 90 \\
\hline Volume $/ \AA^{3}$ & 1745.97(15) \\
\hline $\mathrm{Z}$ & 4 \\
\hline$\rho_{\text {calc }} \mathrm{g} / \mathrm{cm}^{3}$ & 1.422 \\
\hline$\mu / \mathrm{mm}^{-1}$ & 1.415 \\
\hline $\mathrm{F}(000)$ & 784.0 \\
\hline Crystal size $/ \mathrm{mm}^{3}$ & $0.35 \times 0.25 \times 0.25$ \\
\hline Radiation & $\operatorname{MoK} \alpha(\lambda=0.71073)$ \\
\hline \multicolumn{2}{|c|}{$2 \Theta$ range for data collection $/{ }^{\circ} 5.924$ to 55.018} \\
\hline Index ranges & $-12 \leq \mathrm{h} \leq 13,-22 \leq \mathrm{k} \leq 22,-12 \leq 1 \leq 10$ \\
\hline Reflections collected & 21999 \\
\hline Independent reflections & $3999\left[R_{\text {int }}=0.0186, R_{\text {sigma }}=0.0126\right]$ \\
\hline Data/restraints/parameters & 3999/0/218 \\
\hline Goodness-of-fit on $\mathrm{F}^{2}$ & 1.021 \\
\hline Final $R$ indexes $[\mathrm{I}>=2 \sigma(\mathrm{I})]$ & $\mathrm{R}_{1}=0.0257, \mathrm{wR}_{2}=0.0683$ \\
\hline Final $\mathrm{R}$ indexes [all data] & $\mathrm{R}_{1}=0.0286, \mathrm{wR}_{2}=0.0702$ \\
\hline \multicolumn{2}{|c|}{ Largest diff. peak/hole / e $\AA^{-3} 0.59 /-0.24$} \\
\hline $\mathrm{CCDC}$ & 1868747 \\
\hline
\end{tabular}




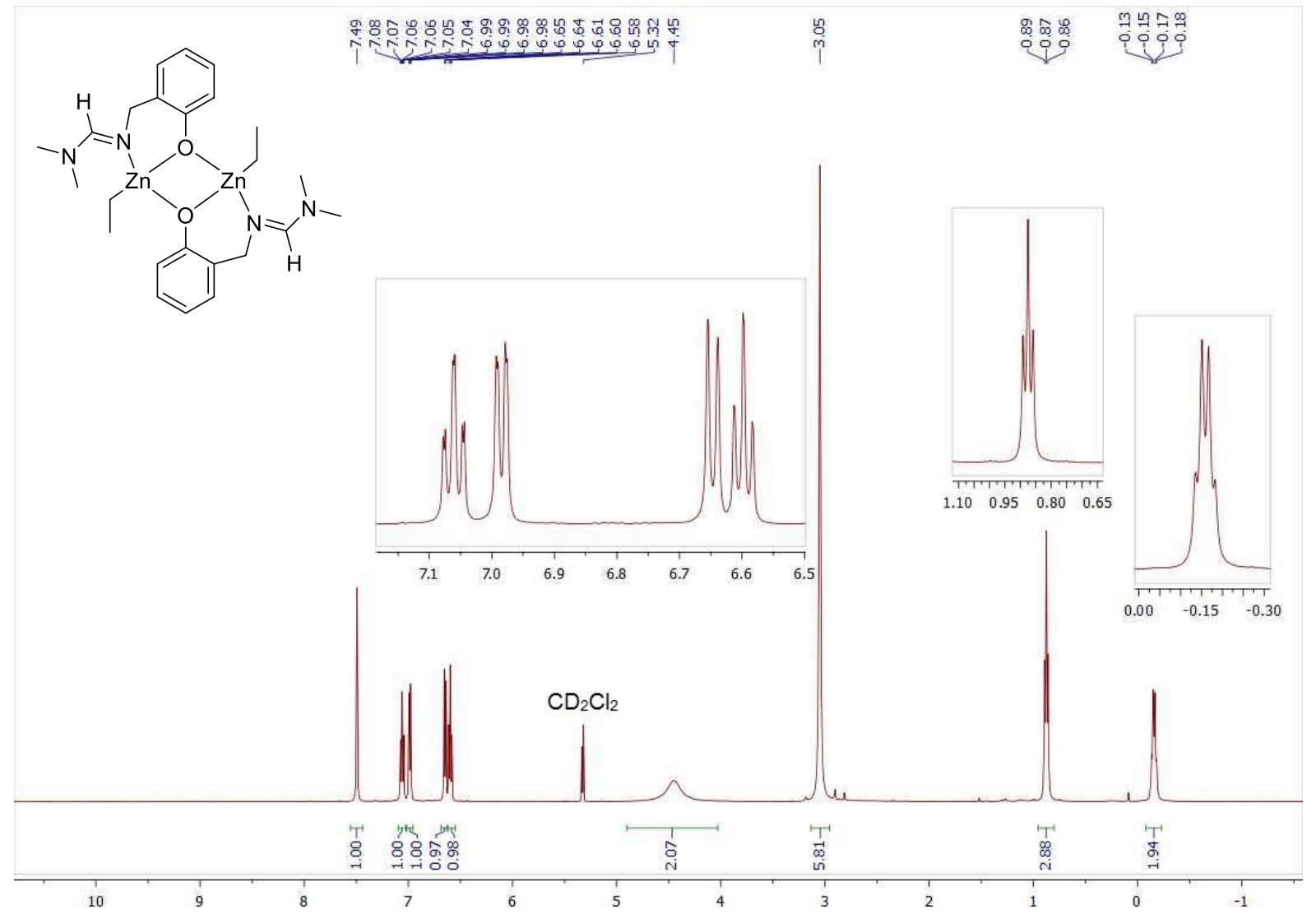

Figure S58: ${ }^{1} \mathrm{H}$ NMR $\left(500 \mathrm{MHz}, \mathrm{CD}_{2} \mathrm{Cl}_{2}\right)$ of $\mathbf{3 a}$

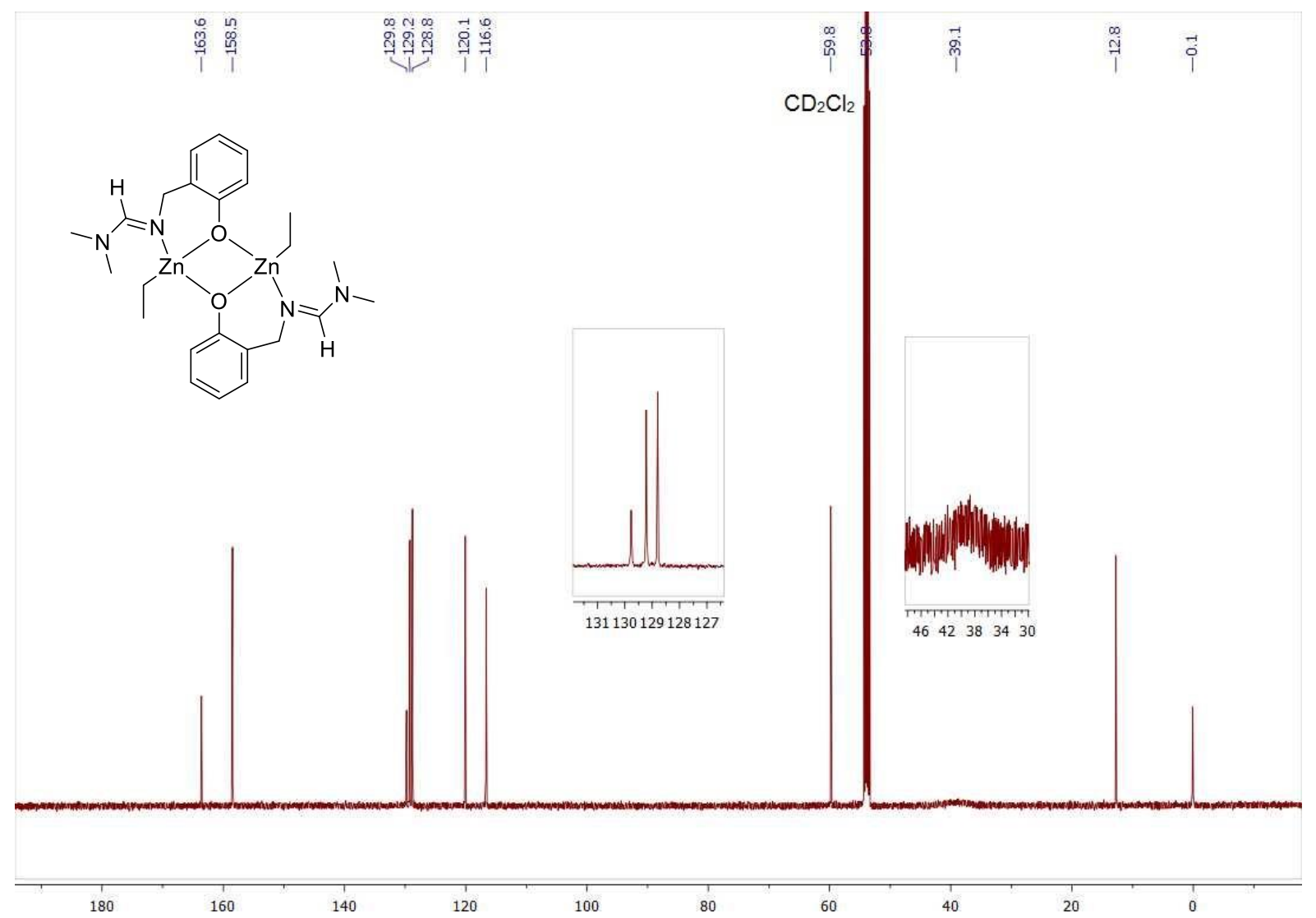

Figure S59: ${ }^{13} \mathrm{C}\left\{{ }^{1} \mathrm{H}\right\} \mathrm{NMR}\left(126 \mathrm{MHz}, \mathrm{CD}_{2} \mathrm{Cl}_{2}\right)$ of 3a 


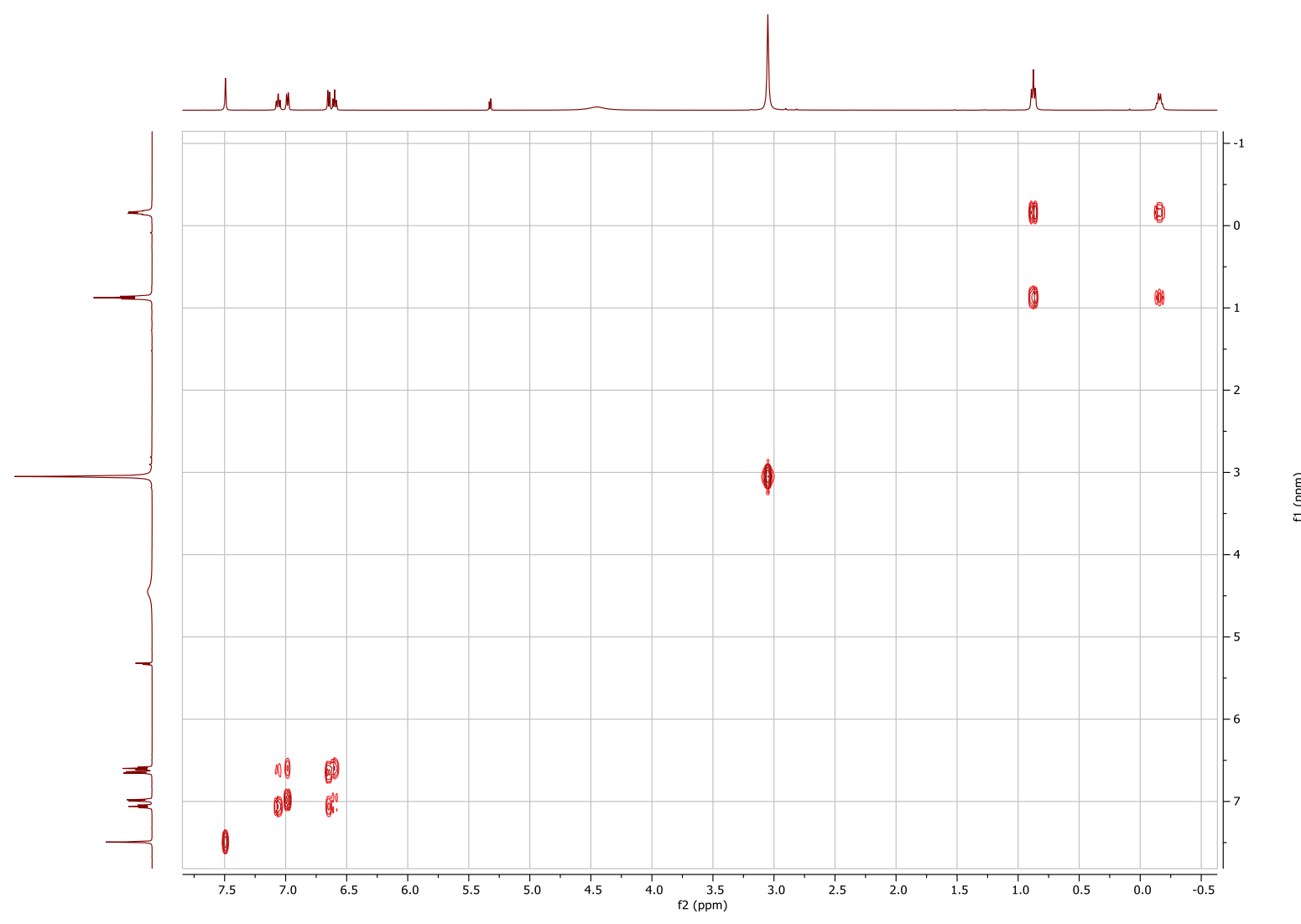

Figure S60: ${ }^{1} \mathrm{H}^{1} \mathrm{H}$ COSY $\left(500 \mathrm{MHz}, \mathrm{CD}_{2} \mathrm{Cl}_{2}\right)$ of $\mathbf{3 a}$

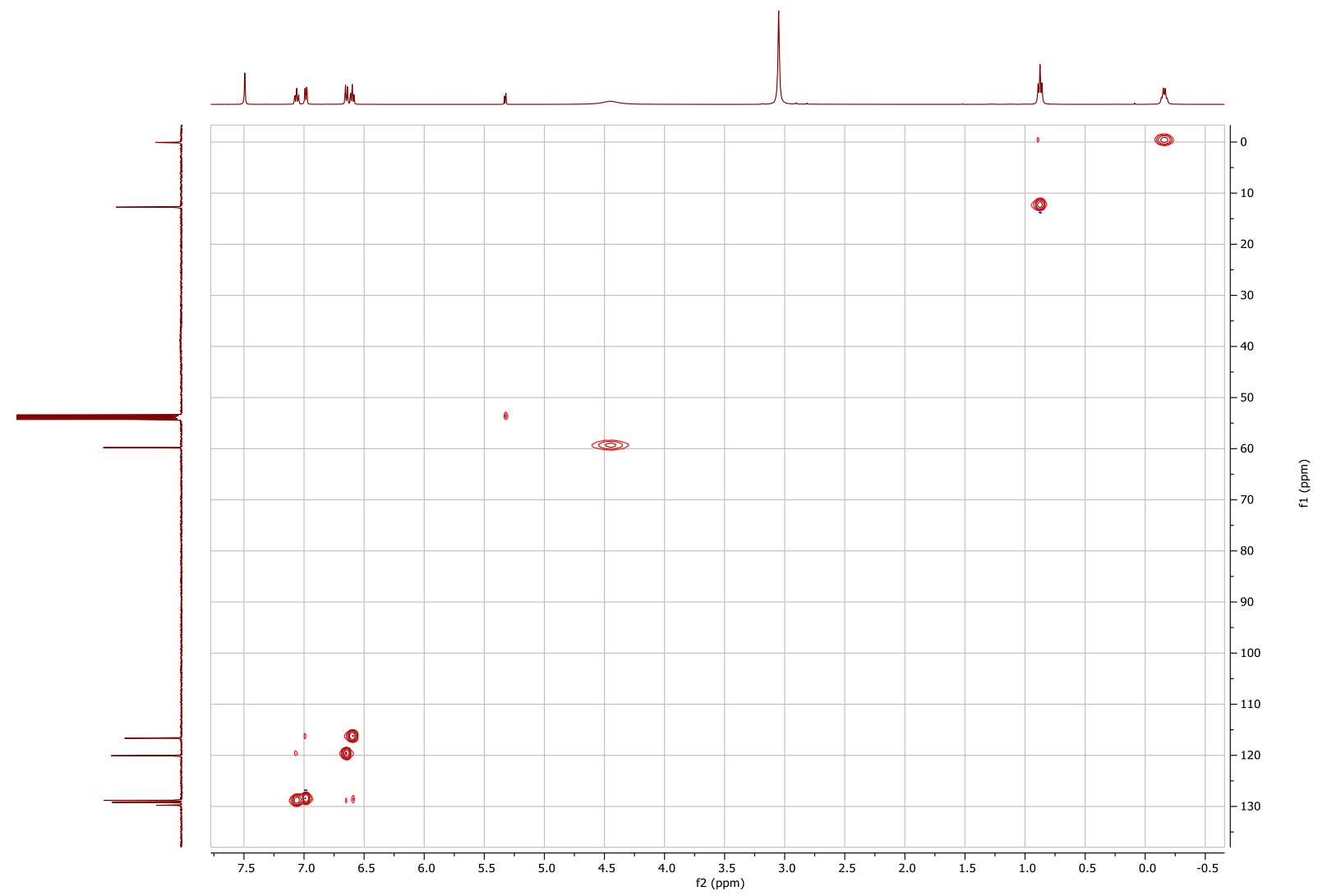

Figure S61: ${ }^{1} \mathrm{H}^{13} \mathrm{C} \mathrm{HSQC}\left(500 \mathrm{MHz} / 126 \mathrm{MHz}, \mathrm{CD}_{2} \mathrm{Cl}_{2}\right)$ of $\mathbf{3 a}$ 


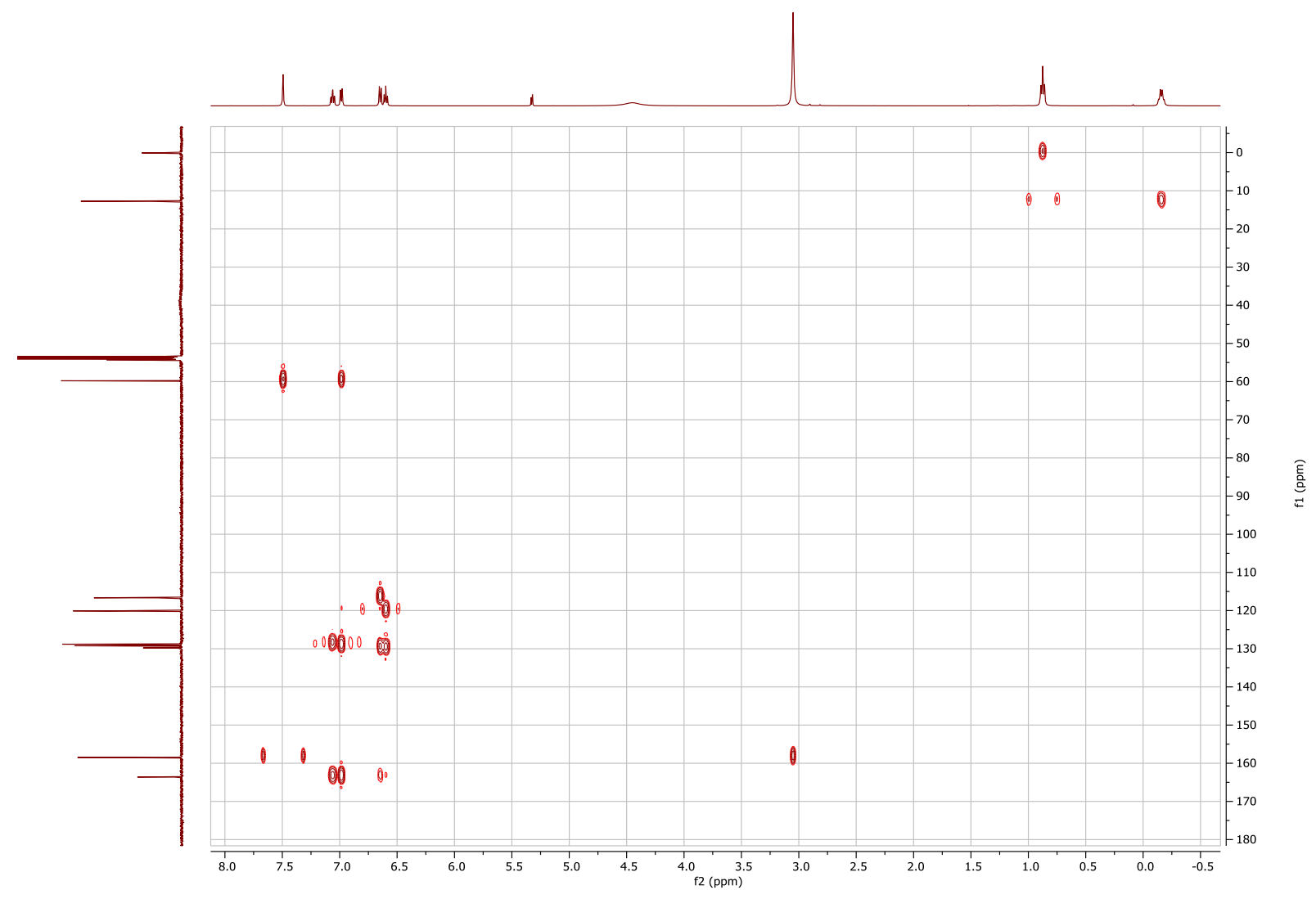

Figure S62: ${ }^{1} \mathrm{H}^{13} \mathrm{C} \mathrm{HMBC}\left(500 \mathrm{MHz} / 126 \mathrm{MHz}, \mathrm{CD}_{2} \mathrm{Cl}_{2}\right)$ of $\mathbf{3 a}$

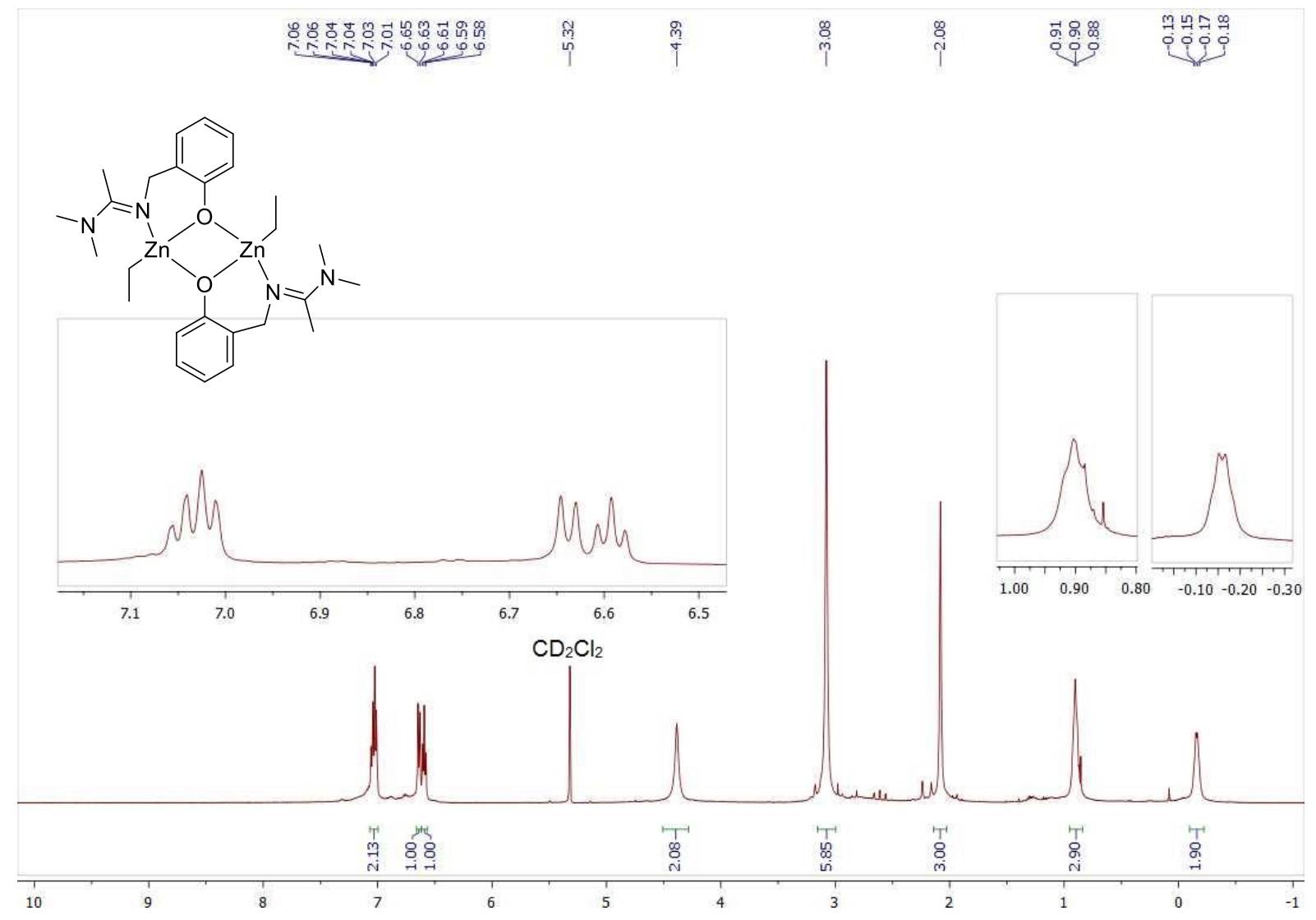

Figure S63: ${ }^{1} \mathrm{H}$ NMR $\left(500 \mathrm{MHz}, \mathrm{CD}_{2} \mathrm{Cl}_{2}\right)$ of $\mathbf{4 a}$ 


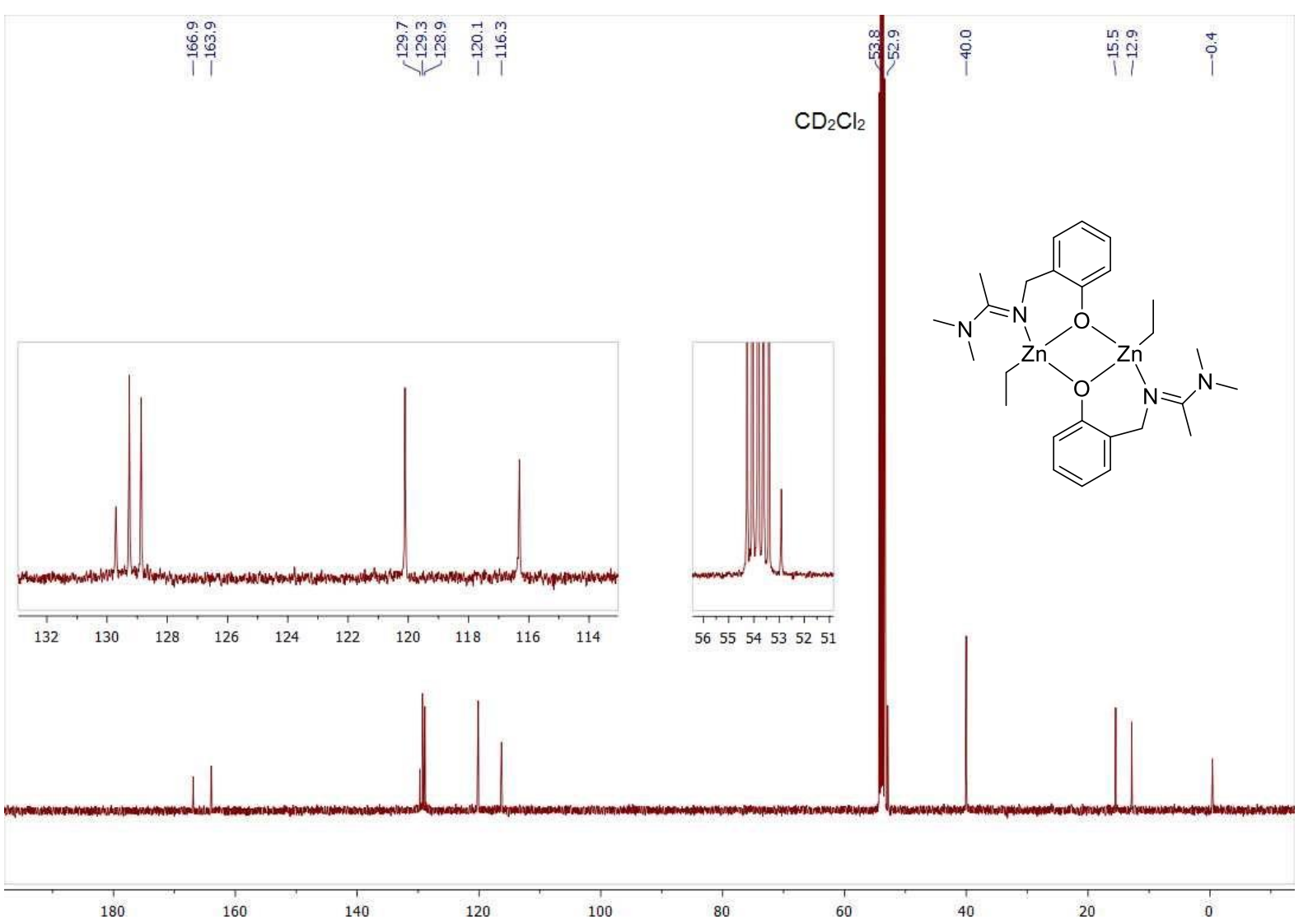

Figure S64: ${ }^{13} \mathrm{C}\left\{{ }^{1} \mathrm{H}\right\}$ NMR $\left(126 \mathrm{MHz}, \mathrm{CD}_{2} \mathrm{Cl}_{2}\right)$ of $\mathbf{4 a}$

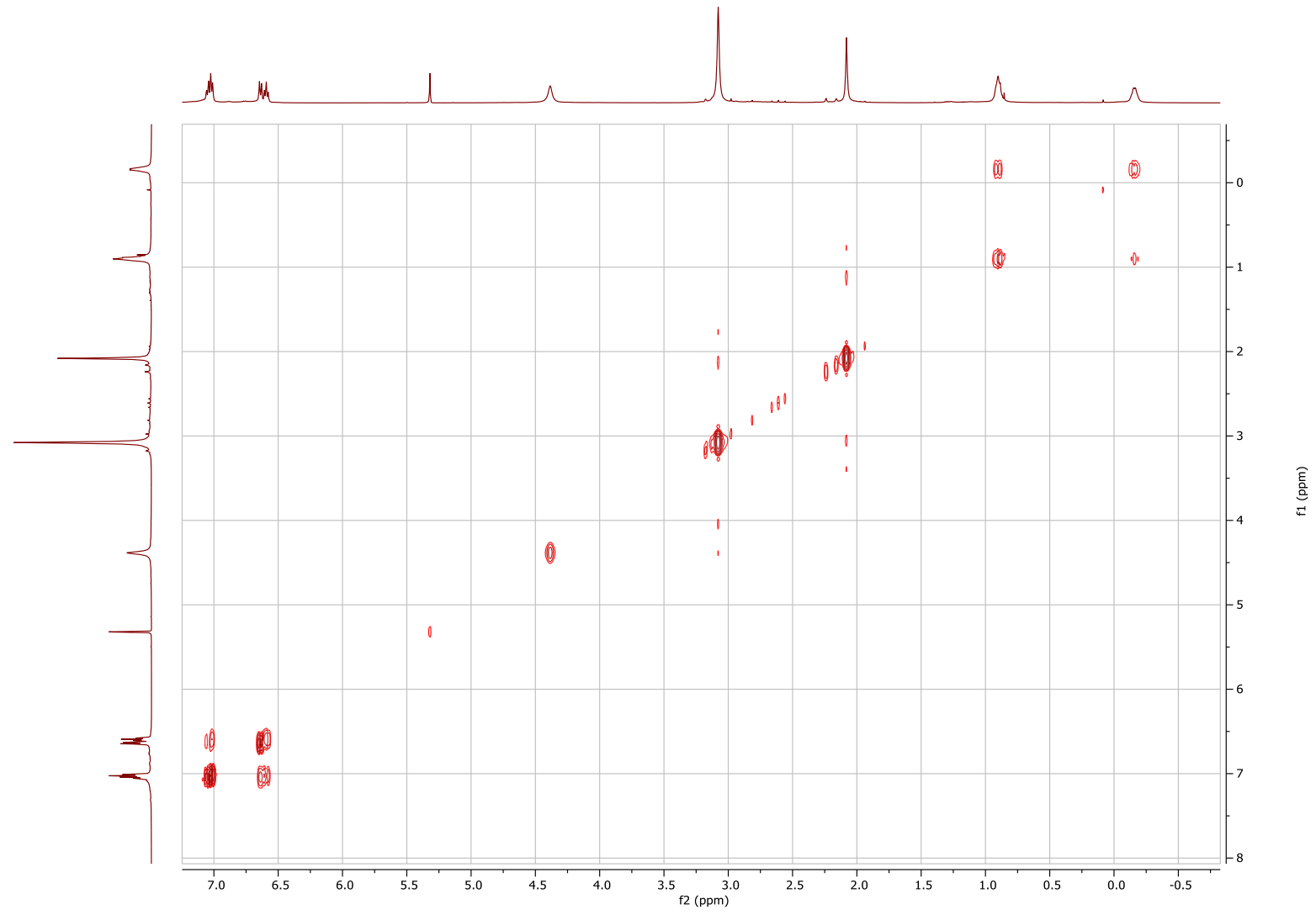

Figure S65: ${ }^{1} \mathrm{H}^{1} \mathrm{H} \operatorname{COSY}\left(500 \mathrm{MHz}, \mathrm{CD}_{2} \mathrm{Cl}_{2}\right)$ of $\mathbf{4 a}$ 


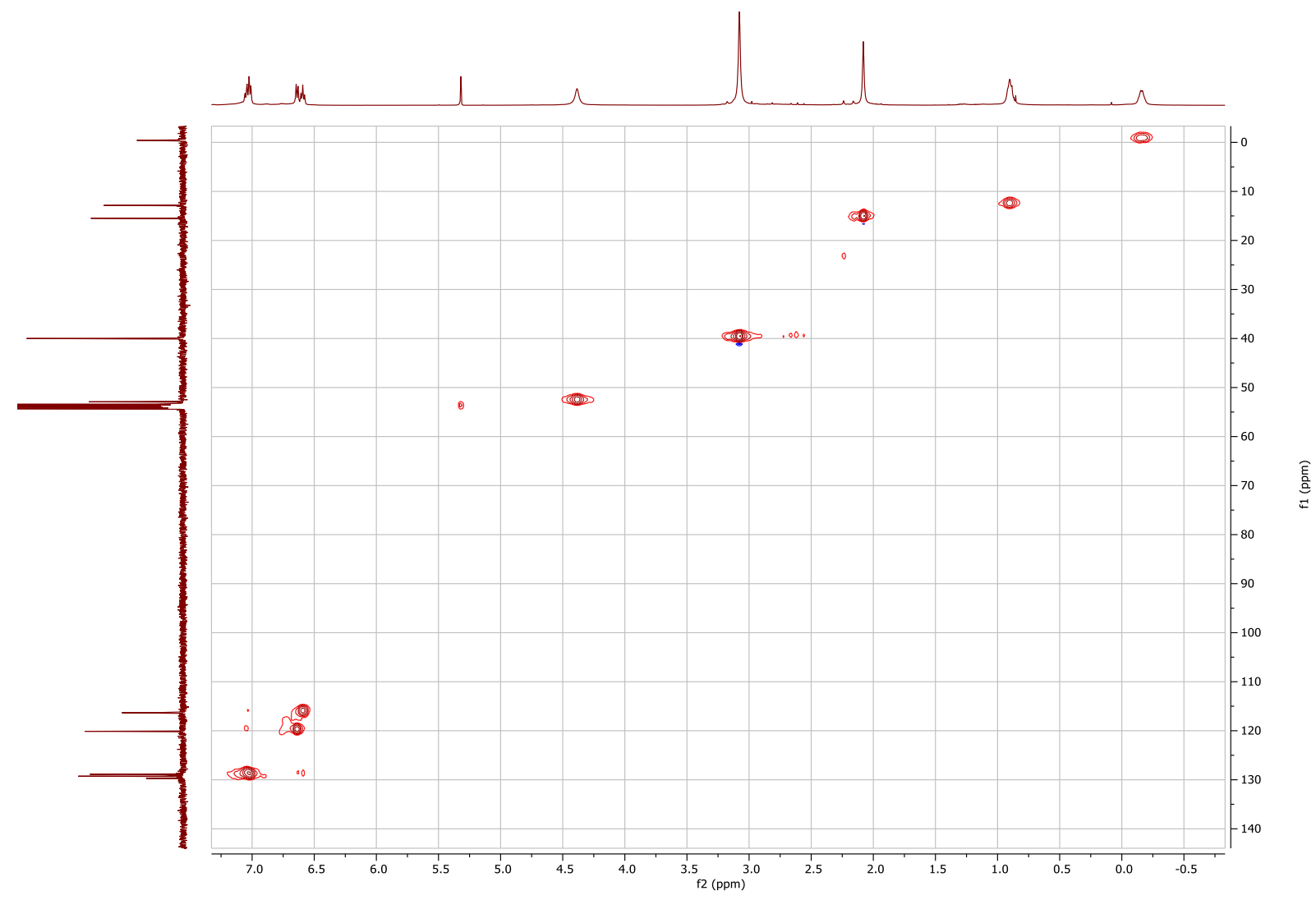

Figure S66: ${ }^{1} \mathrm{H}^{13} \mathrm{C} \mathrm{HSQC}\left(500 \mathrm{MHz} / 126 \mathrm{MHz}, \mathrm{CD}_{2} \mathrm{Cl}_{2}\right)$ of $\mathbf{4 a}$

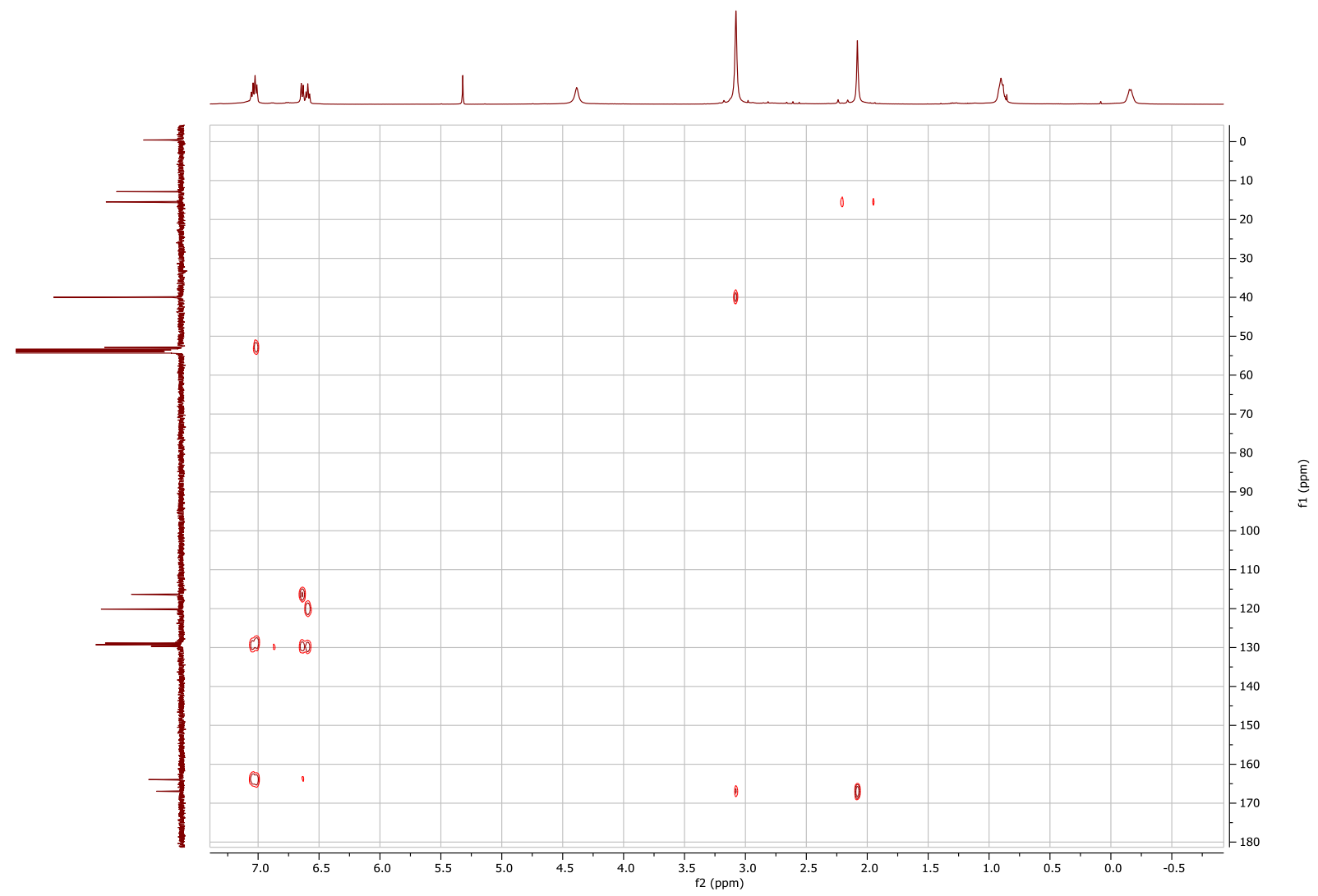

Figure S67: ${ }^{1} \mathrm{H}^{13} \mathrm{C} \mathrm{HMBC}\left(500 \mathrm{MHz} / 126 \mathrm{MHz}, \mathrm{CD}_{2} \mathrm{Cl}_{2}\right)$ of $\mathbf{4 a}$ 


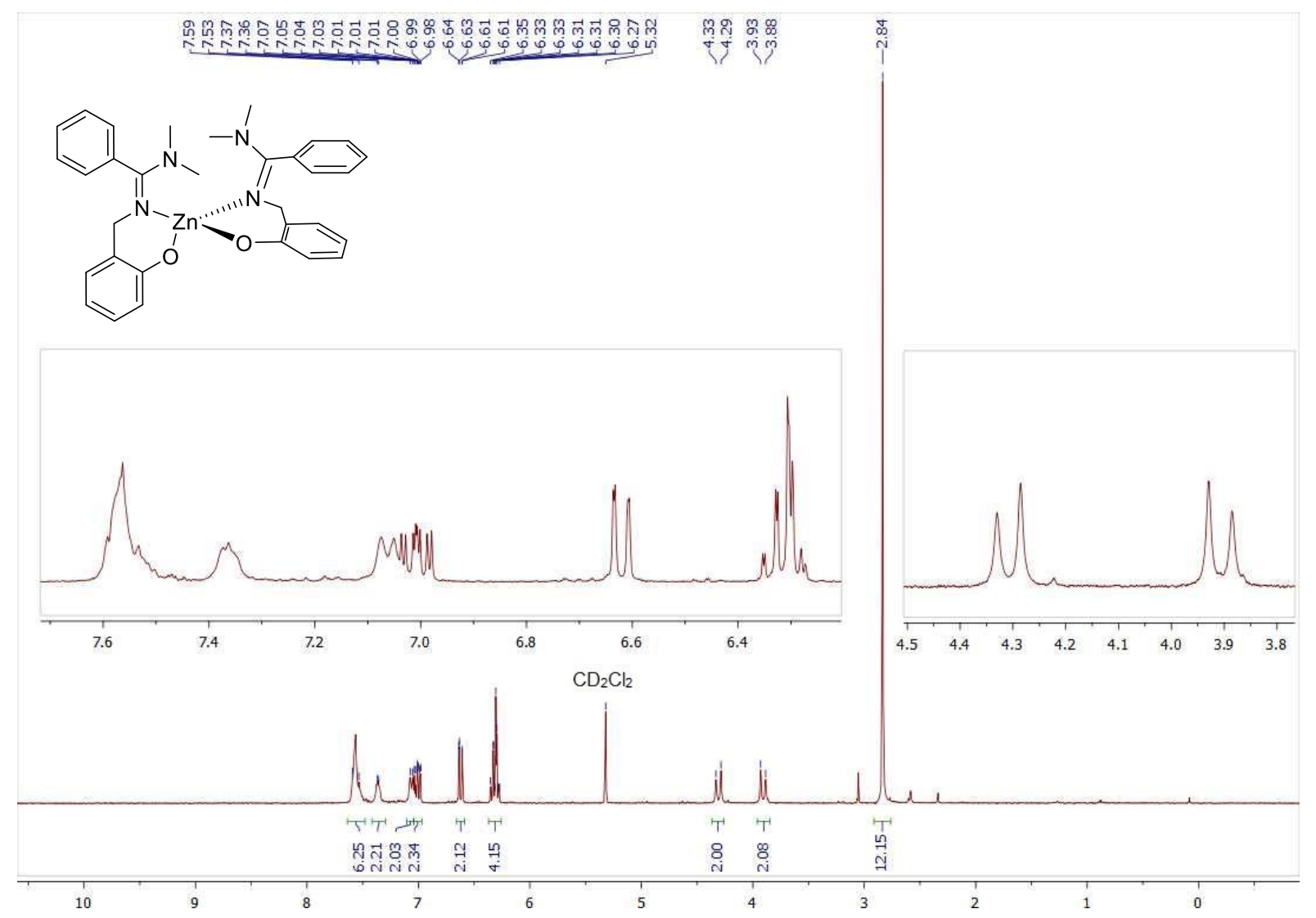

Figure S68: ${ }^{1} \mathrm{H}$ NMR $\left(300 \mathrm{MHz}, \mathrm{CD}_{2} \mathrm{Cl}_{2}\right)$ of 1a'

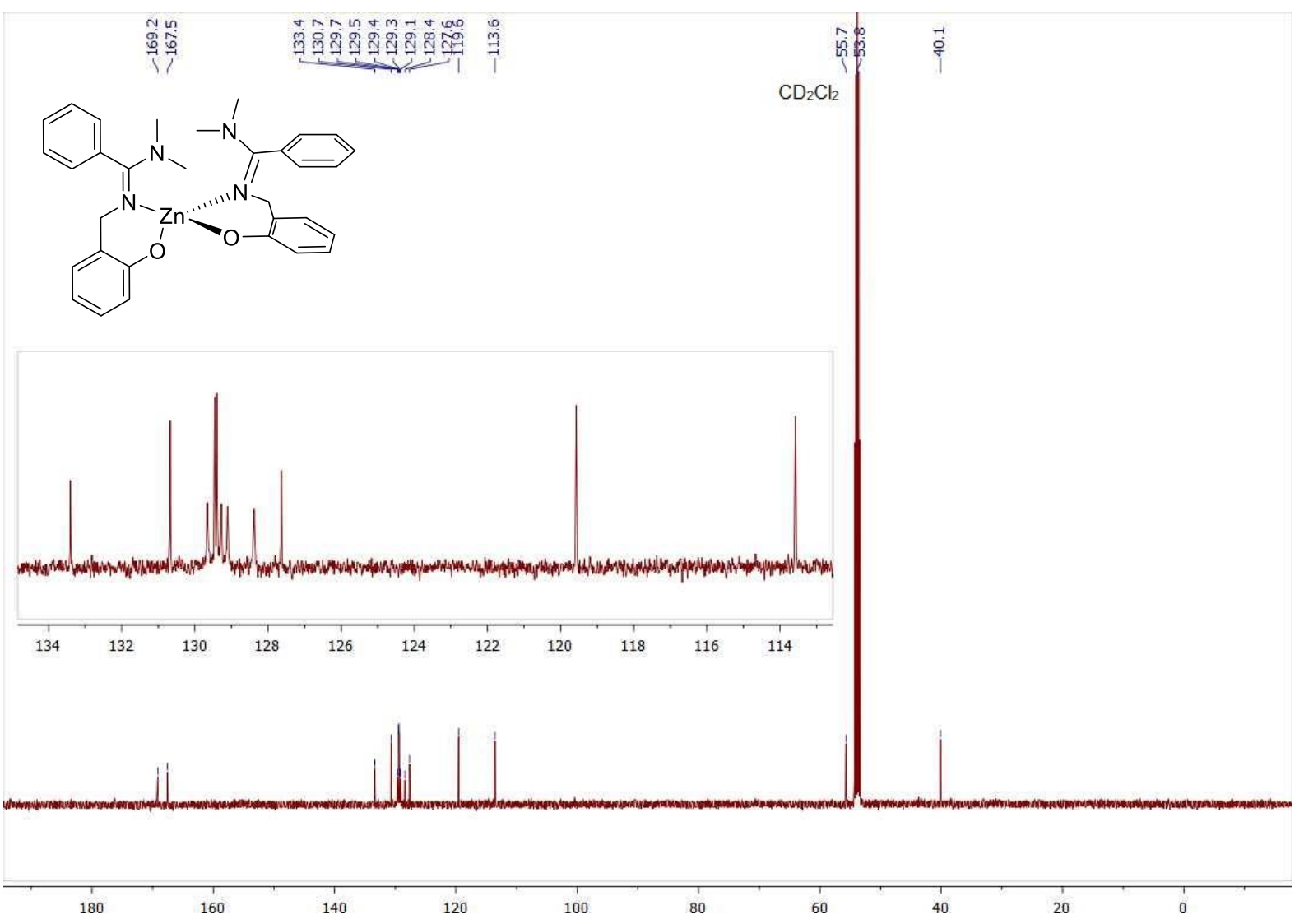

Figure S69: ${ }^{13} \mathrm{C}\left\{{ }^{1} \mathrm{H}\right\}$ NMR $\left(126 \mathrm{MHz}, \mathrm{CD}_{2} \mathrm{Cl}_{2}\right)$ of $\mathbf{1 a}$ ' 


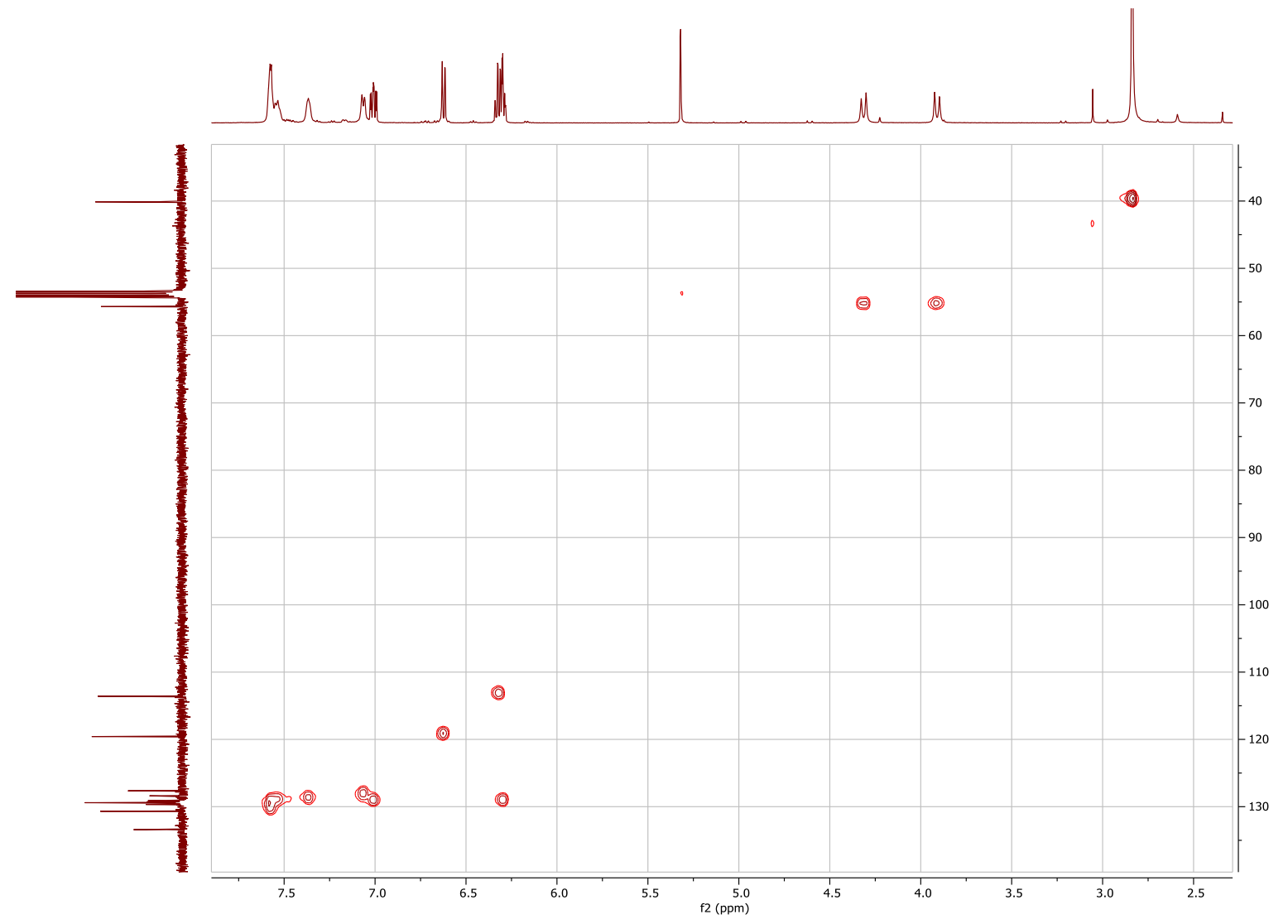

Figure S70: ${ }^{1} \mathrm{H}^{13} \mathrm{C} \mathrm{HSQC}\left(500 \mathrm{MHz} / 126 \mathrm{MHz}, \mathrm{CD}_{2} \mathrm{Cl}_{2}\right)$ of $\mathbf{1 a}$ '

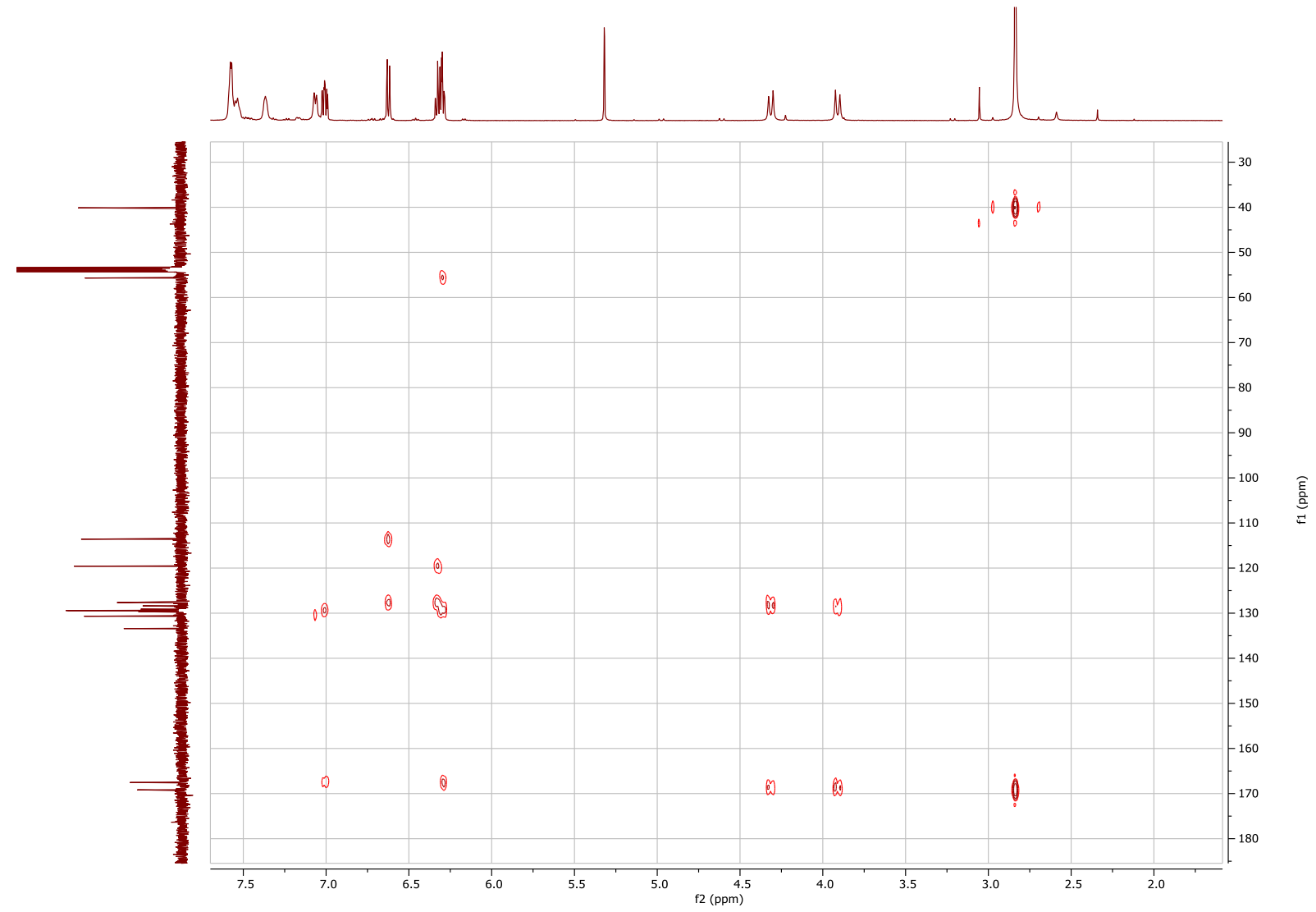

Figure S71: ${ }^{1} \mathrm{H}^{13} \mathrm{C} \mathrm{HMBC}\left(500 \mathrm{MHz} / 126 \mathrm{MHz}, \mathrm{CD}_{2} \mathrm{Cl}_{2}\right)$ of $\mathbf{1 a}$ ' 


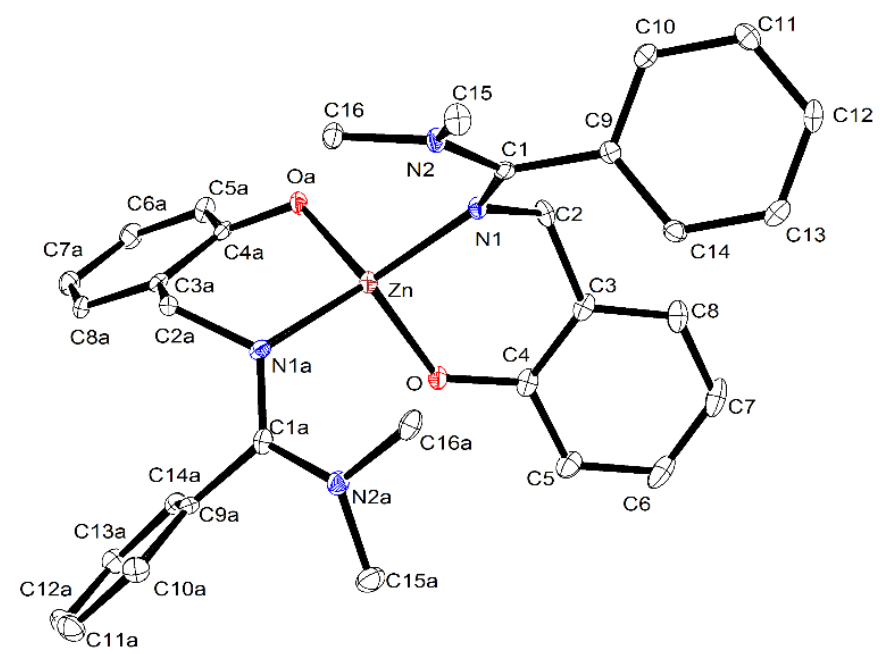

Figure S72: Crystal structure of 1a'

Table S7: Crystal data and structure refinement of 1a'

Identification code in cif

Empirical formula

Formula weight

Temperature/K

Crystal system

Space group

$\mathrm{a} / \AA$

$\mathrm{b} / \AA ̊ \AA$

c/Å

$\alpha /{ }^{\circ}$

$\beta /{ }^{\circ}$

$\gamma /{ }^{\circ}$

Volume $/ \AA^{3}$

Z

$\rho_{\text {calc }} \mathrm{g} / \mathrm{cm}^{3}$

$\mu / \mathrm{mm}^{-1}$

$\mathrm{F}(000)$

Crystal size $/ \mathrm{mm}^{3}$

Radiation

\section{C1a'}

$\mathrm{C}_{32} \mathrm{H}_{34} \mathrm{~N}_{4} \mathrm{O}_{2} \mathrm{Zn}$

572.00

115

monoclinic

$P{ }_{1} / C$

12.754(9)

12.535(9)

18.021(13)

90

94.450(13)

90

2872(3)

4

1.323

0.890

1200.0

$0.3 \times 0.02 \times 0.02$

$\operatorname{MoK} \alpha(\lambda=0.71073)$

$2 \Theta$ range for data collection/ ${ }^{\circ} 6.256$ to 49.99

Index ranges

$-15 \leq \mathrm{h} \leq 15,-14 \leq \mathrm{k} \leq 14,-21 \leq 1 \leq 20$

Reflections collected

33088

Independent reflections

Data/restraints/parameters

$5047\left[R_{\text {int }}=0.1115, R_{\text {sigma }}=0.0849\right]$

Goodness-of-fit on $\mathrm{F}^{2}$

$5047 / 0 / 356$

0.995

Final $R$ indexes $[\mathrm{I}>=2 \sigma(\mathrm{I})] \quad \mathrm{R}_{1}=0.0438, \mathrm{wR}_{2}=0.0676$

Final $\mathrm{R}$ indexes [all data] $\quad \mathrm{R}_{1}=0.0909, \mathrm{wR}_{2}=0.0791$

Largest diff. peak/hole / e $\AA^{-3} 0.34 /-0.32$

CCDC

1868748 


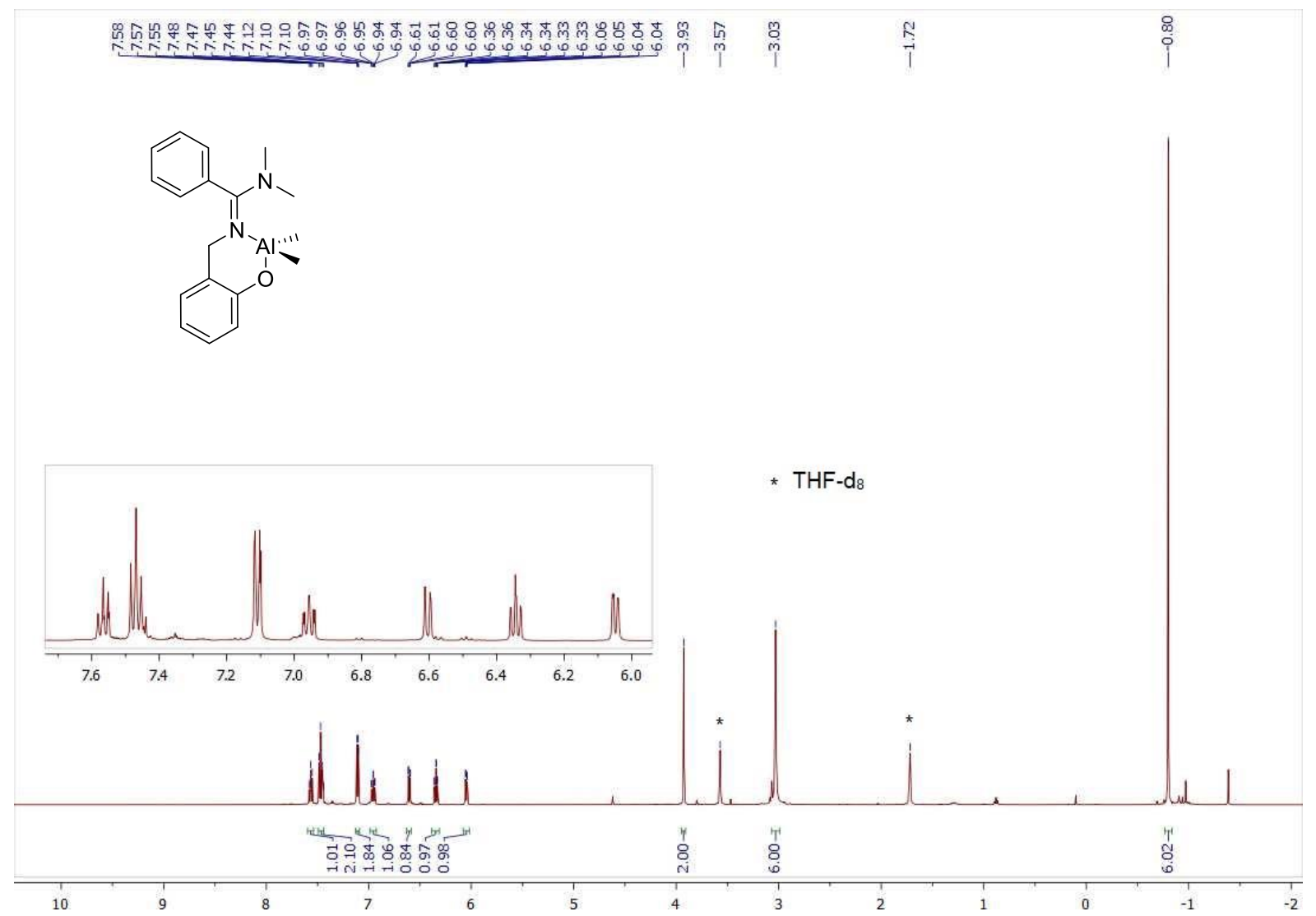

Figure S73: ${ }^{1} \mathrm{H}$ NMR (500 MHz, THF-d8) of $\mathbf{1 b}$

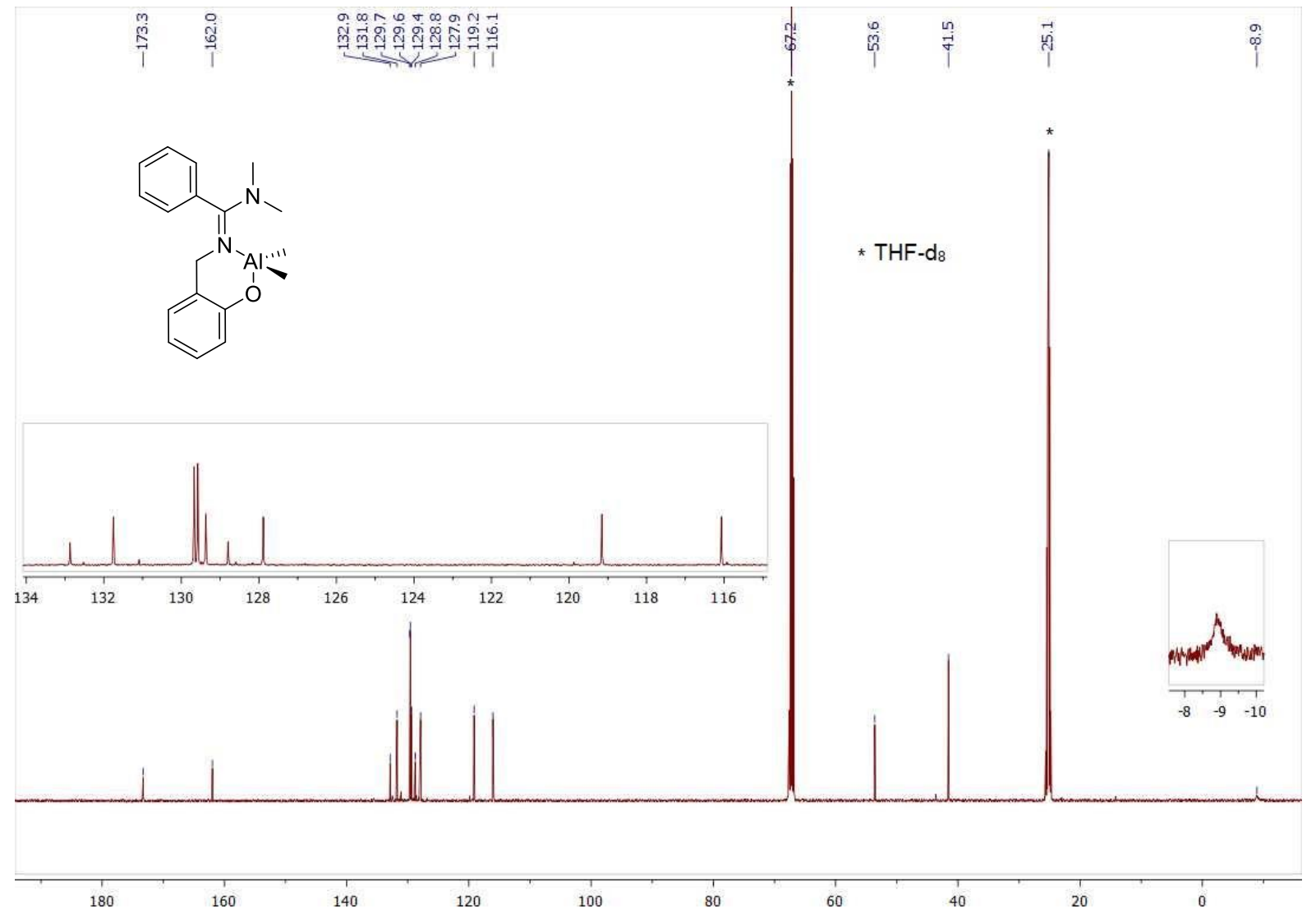

Figure S74: ${ }^{13} \mathrm{C}\left\{{ }^{1} \mathrm{H}\right\}$ NMR $(126 \mathrm{MHz}, \mathrm{THF}-\mathrm{d} 8)$ of $\mathbf{1 b}$ 


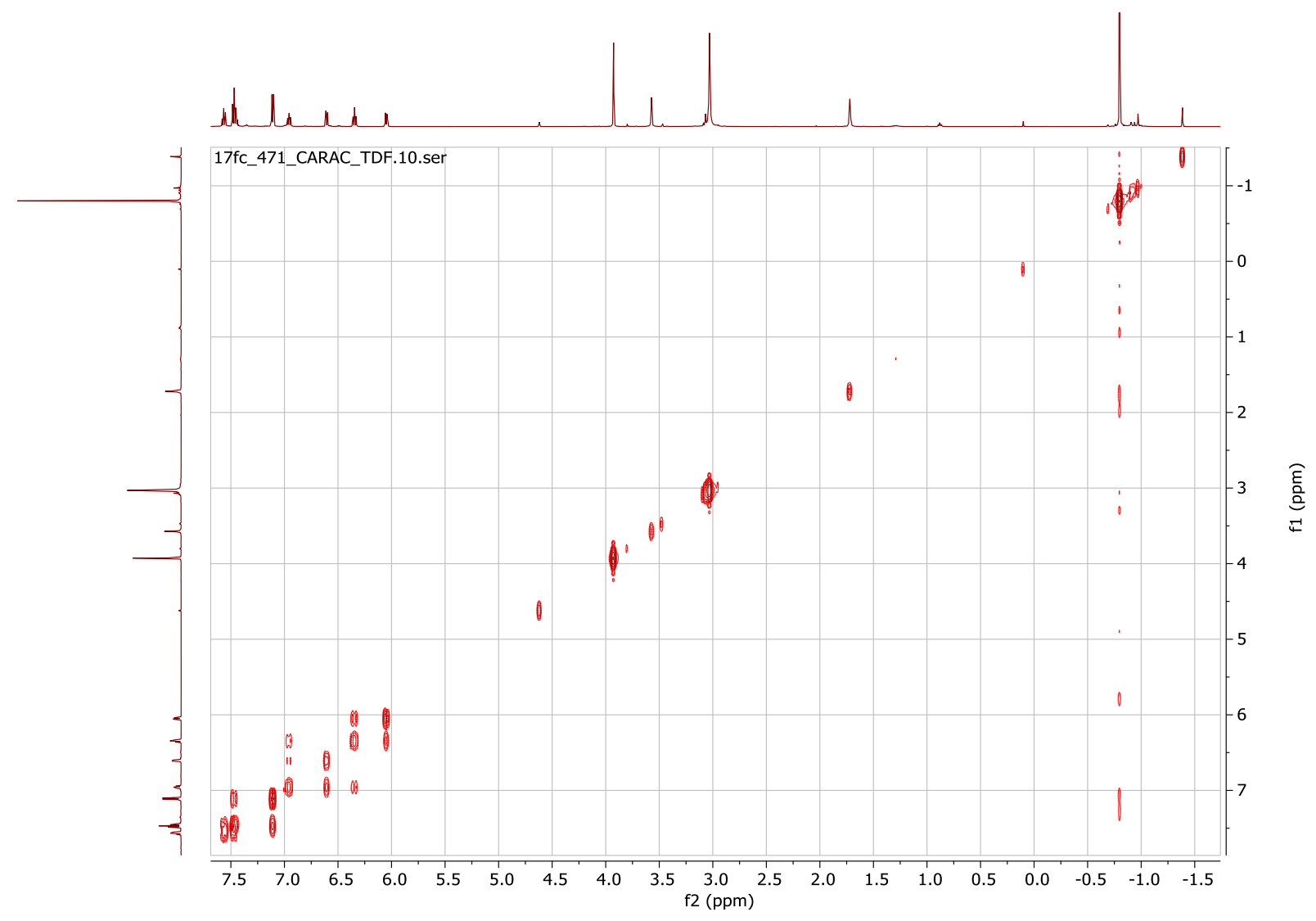

Figure S75: ${ }^{1} \mathrm{H}{ }^{1} \mathrm{H}$ COSY (500 MHz, THF-d8) of $\mathbf{1 b}$

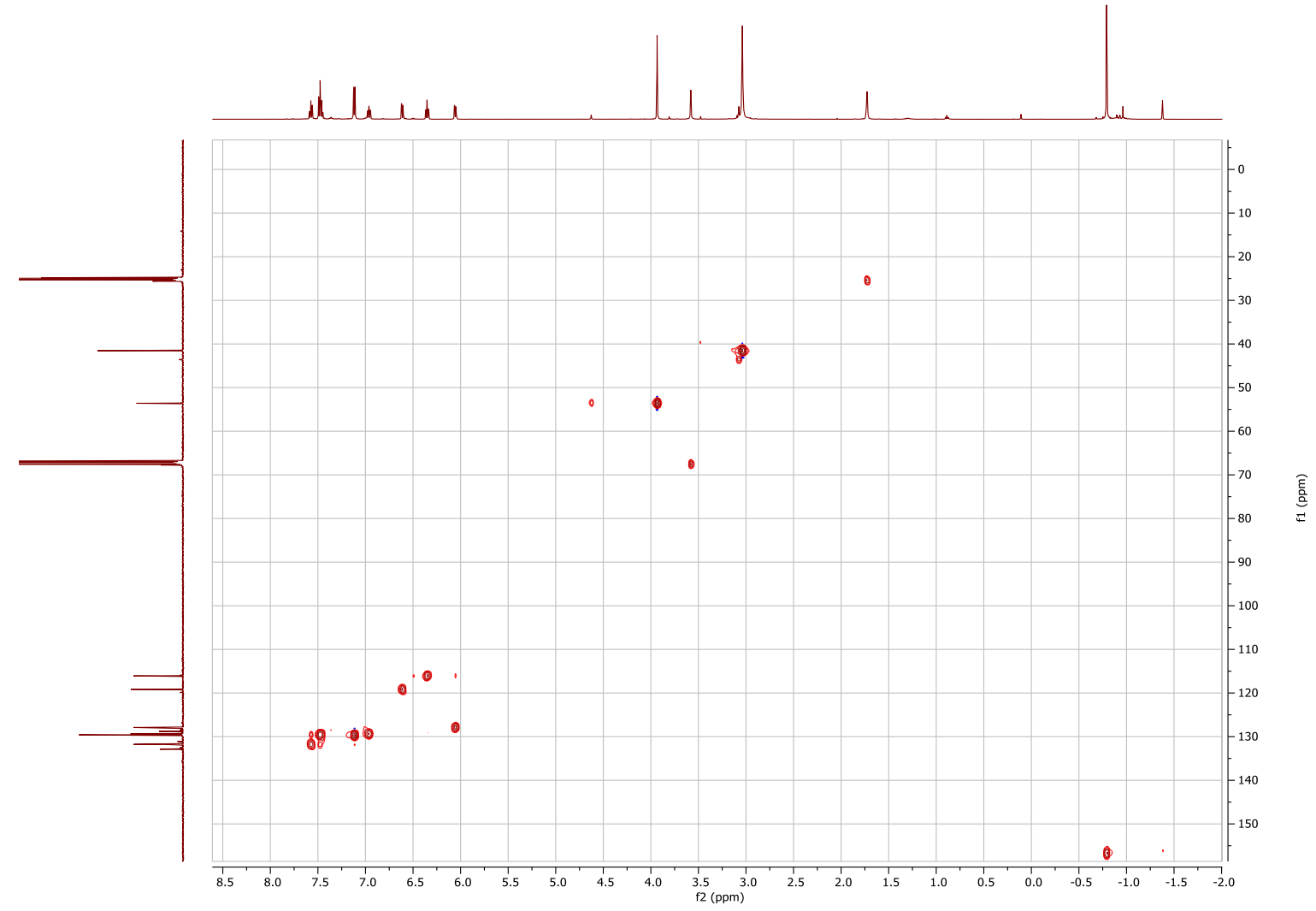

Figure S76: ${ }^{1} \mathrm{H}^{13} \mathrm{C}$ HSQC (500 MHz / $\left.126 \mathrm{MHz}, \mathrm{THF}-\mathrm{d}_{8}\right)$ of $\mathbf{1 b}$ 


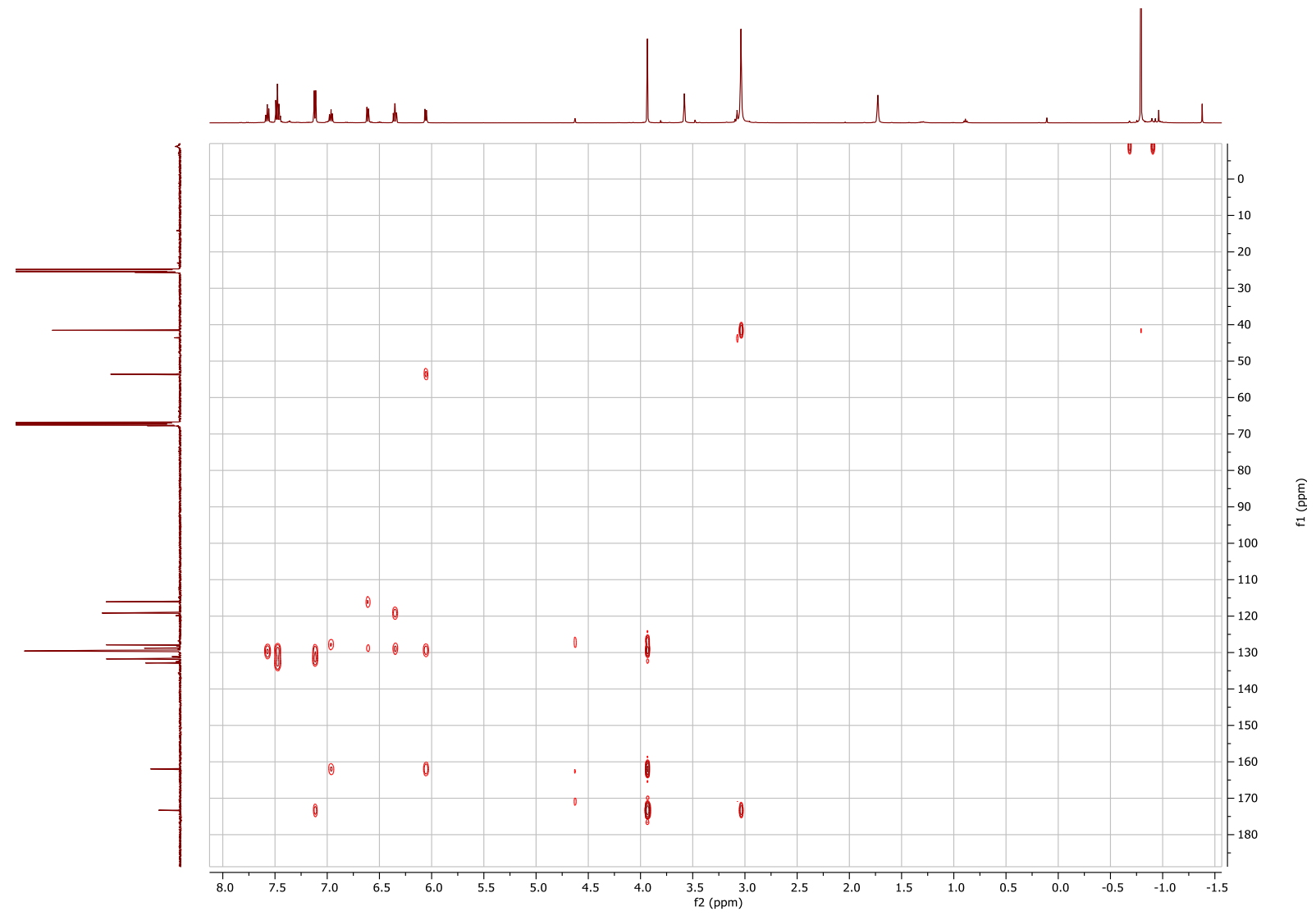

Figure S77: ${ }^{1} \mathrm{H}^{13} \mathrm{C} \mathrm{HMBC}(500 \mathrm{MHz} / 126 \mathrm{MHz}, \mathrm{THF}-\mathrm{d} 8)$ of $\mathbf{1 b}$

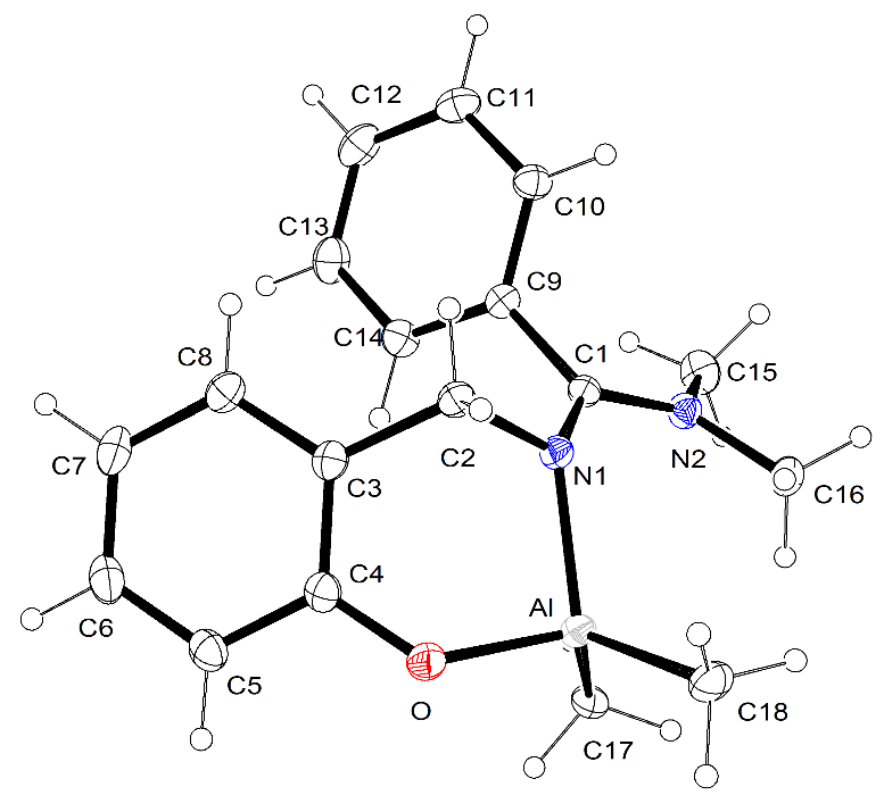

Figure S78: Crystal structure of $\mathbf{1 b}$ 
Table S8: Crystal data and structure refinement of $\mathbf{1 b}$

\begin{tabular}{|c|c|}
\hline Identification code in cif & C1b \\
\hline Empirical formula & $\mathrm{C}_{18} \mathrm{H}_{23} \mathrm{AlN}_{2} \mathrm{O}$ \\
\hline Formula weight & 310.36 \\
\hline Temperature/K & 150.0 \\
\hline Crystal system & monoclinic \\
\hline Space group & $P 2{ }_{1} / c$ \\
\hline $\mathrm{a} / \AA$ & $9.917(3)$ \\
\hline $\mathrm{b} / \AA$ & $13.642(3)$ \\
\hline $\mathrm{c} / \AA ̊$ & $13.468(3)$ \\
\hline$\alpha /^{\circ}$ & 90 \\
\hline$\beta /{ }^{\circ}$ & $104.255(10)$ \\
\hline$\gamma /{ }^{\circ}$ & 90 \\
\hline Volume $/ \AA^{3}$ & $1766.0(7)$ \\
\hline $\mathrm{Z}$ & 4 \\
\hline$\rho_{\text {calc }} \mathrm{g} / \mathrm{cm}^{3}$ & 1.167 \\
\hline$\mu / \mathrm{mm}^{-1}$ & 0.118 \\
\hline $\mathrm{F}(000)$ & 664.0 \\
\hline Crystal size $/ \mathrm{mm}^{3}$ & $0.25 \times 0.25 \times 0.25$ \\
\hline Radiation & $\operatorname{MoK} \alpha(\lambda=0.71073)$ \\
\hline \multicolumn{2}{|c|}{$2 \Theta$ range for data collection $/{ }^{\circ} 5.972$ to 54.878} \\
\hline Index ranges & $-12 \leq \mathrm{h} \leq 12,-17 \leq \mathrm{k} \leq 17,-13 \leq 1 \leq 17$ \\
\hline Reflections collected & 27446 \\
\hline Independent reflections & $4018\left[R_{\text {int }}=0.0391, R_{\text {sigma }}=0.0310\right]$ \\
\hline Data/restraints/parameters & $4018 / 0 / 203$ \\
\hline Goodness-of-fit on $\mathrm{F}^{2}$ & 1.062 \\
\hline Final R indexes $[\mathrm{I}>=2 \sigma(\mathrm{I})]$ & $\mathrm{R}_{1}=0.0417, \mathrm{wR}_{2}=0.0999$ \\
\hline Final $\mathrm{R}$ indexes [all data] & $\mathrm{R}_{1}=0.0667, \mathrm{wR}_{2}=0.1146$ \\
\hline \multicolumn{2}{|c|}{ Largest diff. peak/hole / e $\AA^{-3} 0.28 /-0.32$} \\
\hline $\mathrm{CCDC}$ & 1868749 \\
\hline
\end{tabular}




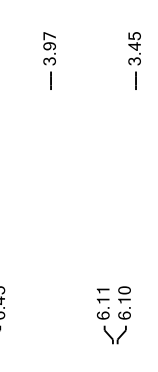
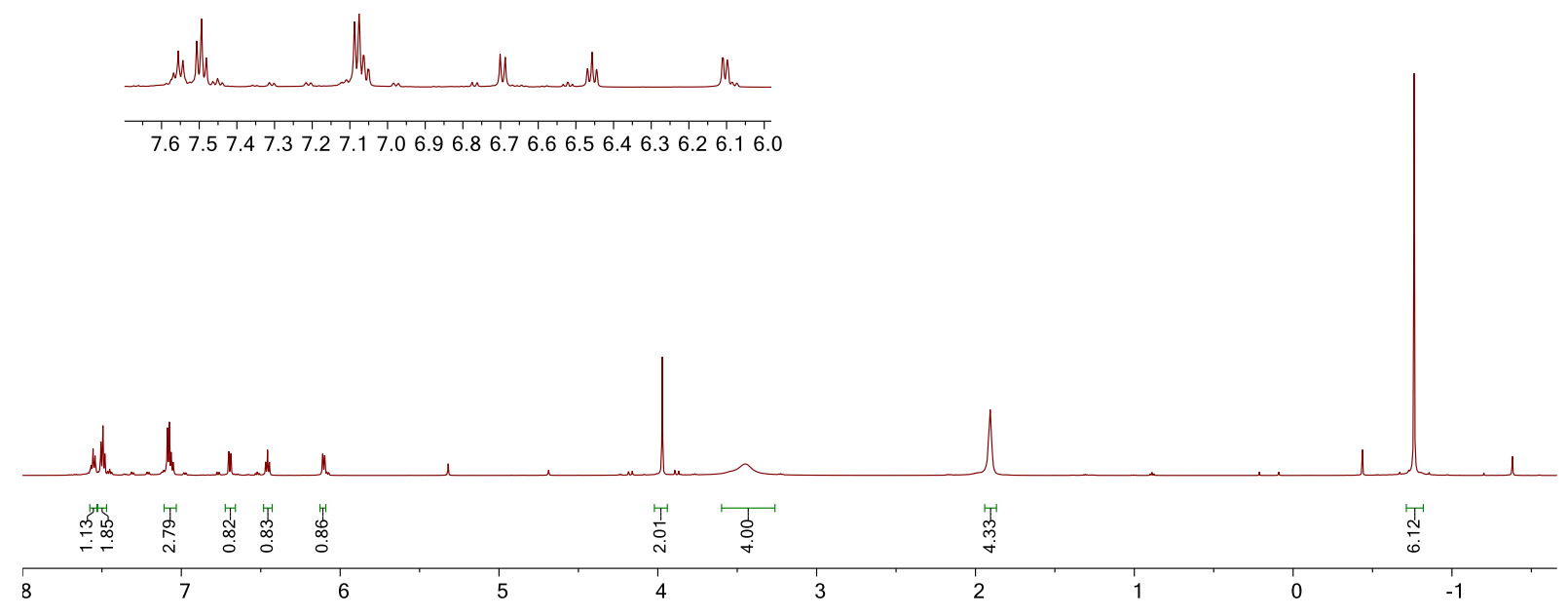

Figure S79: ${ }^{1} \mathrm{H}$ NMR $\left(600 \mathrm{MHz}, \mathrm{CD}_{2} \mathrm{Cl}_{2}\right)$ of $\mathbf{2 b}$
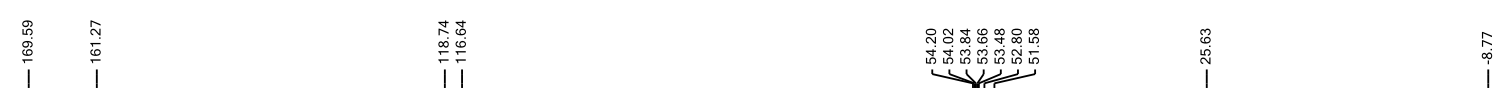

I
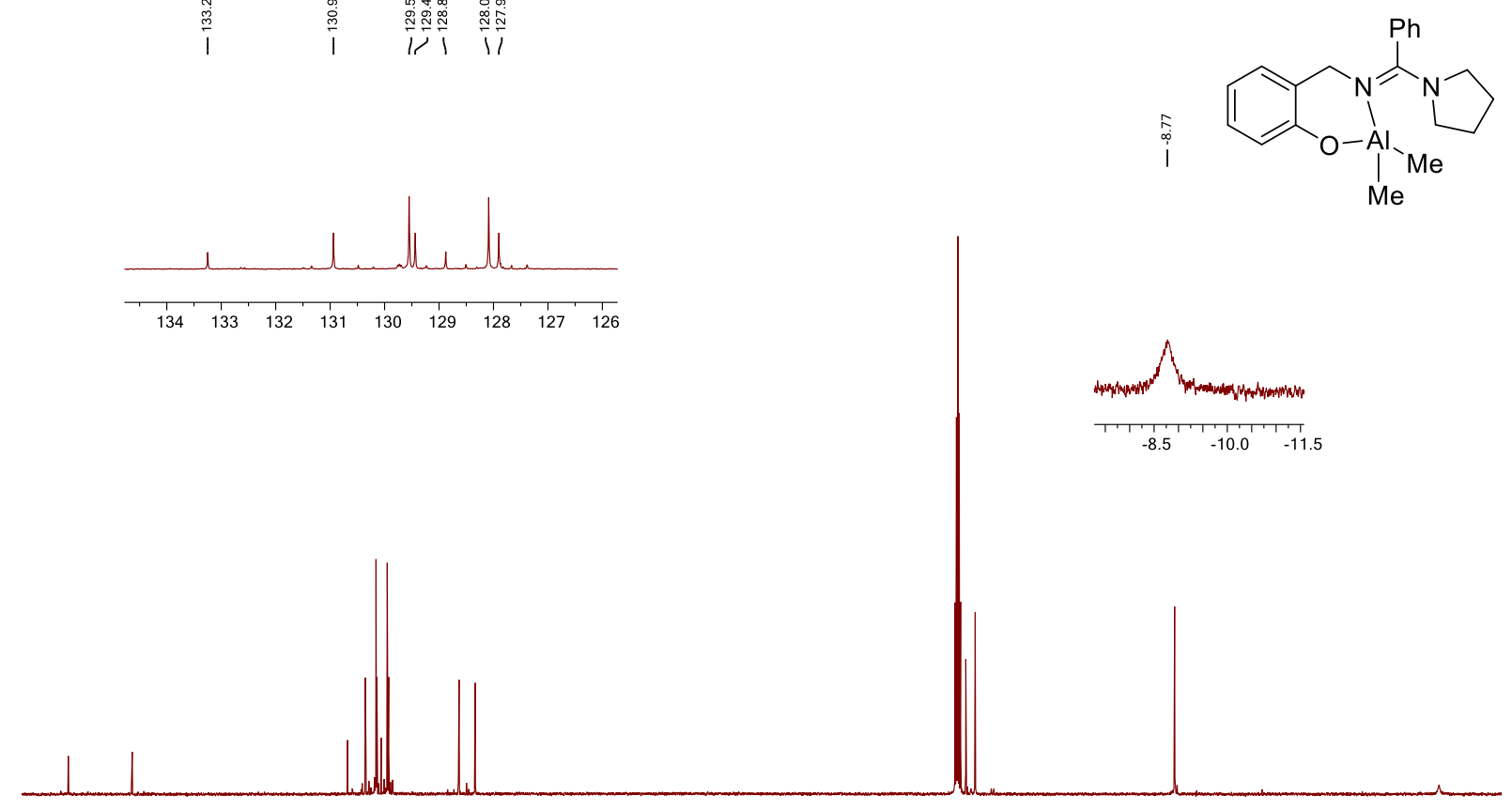

160

120

100

80

40

20

0

Figure S80: ${ }^{13} \mathrm{C}\left\{{ }^{1} \mathrm{H}\right\}$ NMR $\left(151 \mathrm{MHz}, \mathrm{CD}_{2} \mathrm{Cl}_{2}\right)$ of $\mathbf{2 b}$ 


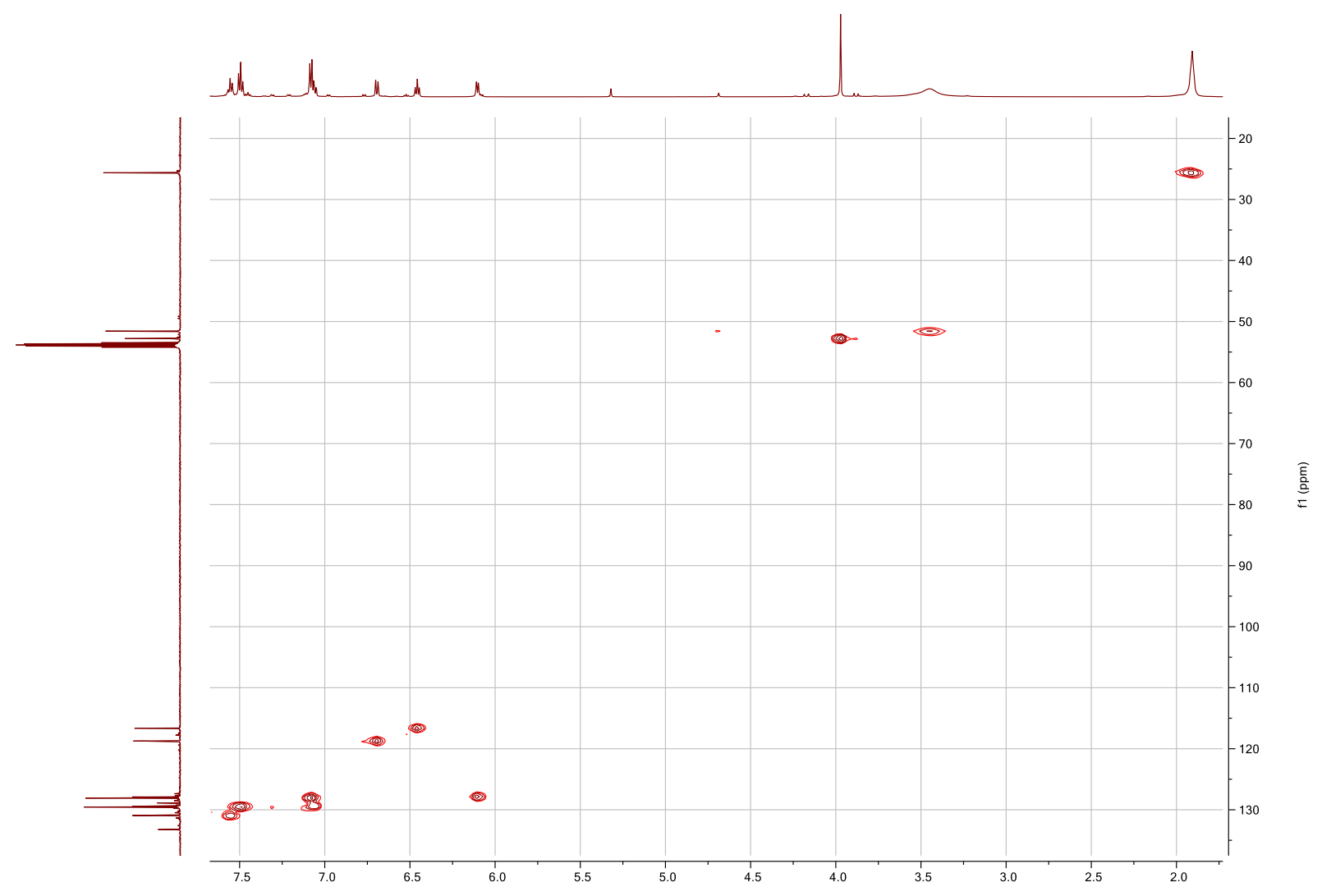

Figure S81: ${ }^{1} \mathrm{H}^{13} \mathrm{C}$ HSQC $\left(600 \mathrm{MHz} / 151 \mathrm{MHz}, \mathrm{CD}_{2} \mathrm{Cl}_{2}\right)$ of $\mathbf{2 b}$

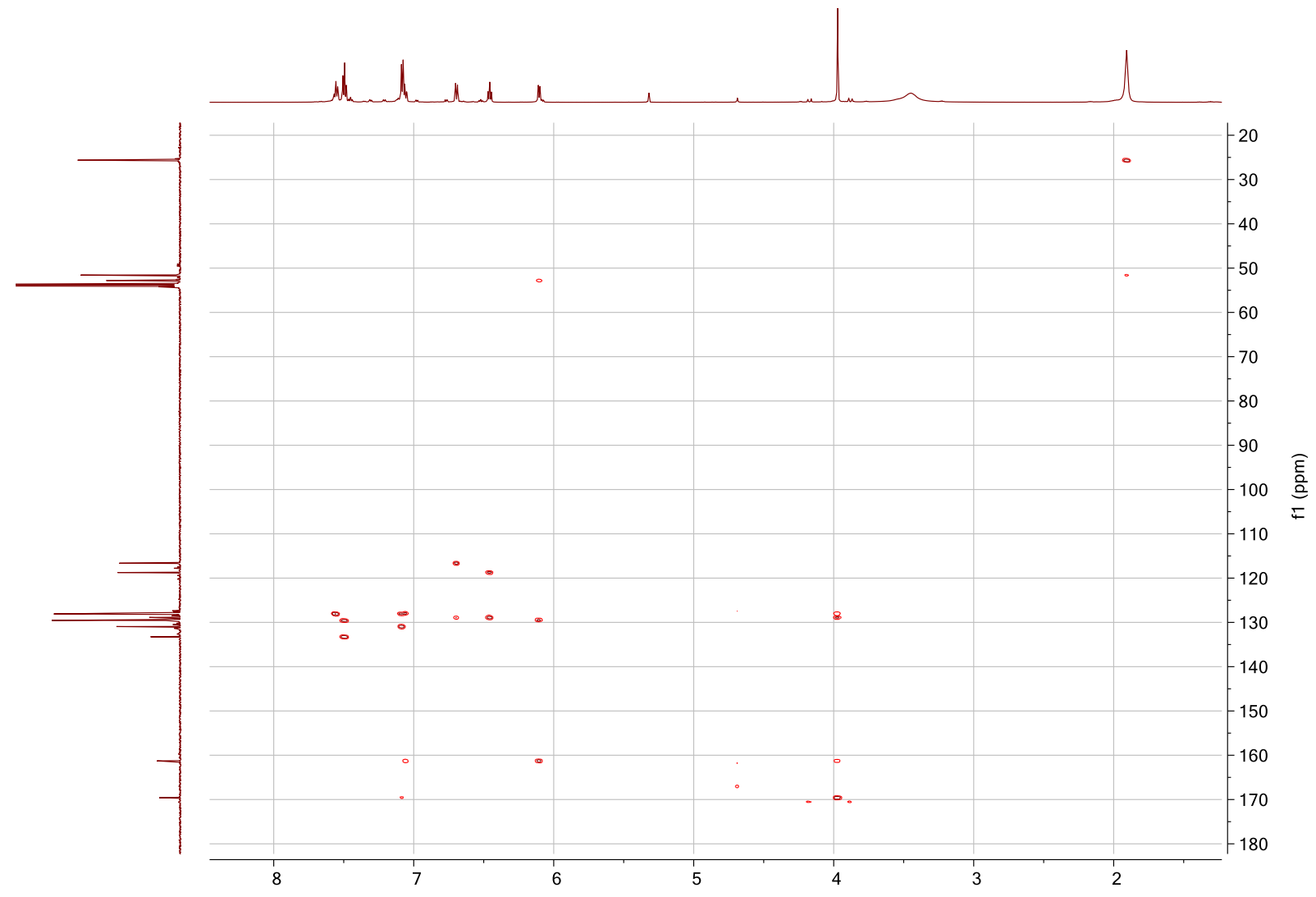

Figure S82: ${ }^{1} \mathrm{H}^{13} \mathrm{C} \mathrm{HMBC}\left(600 \mathrm{MHz} / 151 \mathrm{MHz}, \mathrm{CD}_{2} \mathrm{Cl}_{2}\right)$ of $\mathbf{2 b}$ 


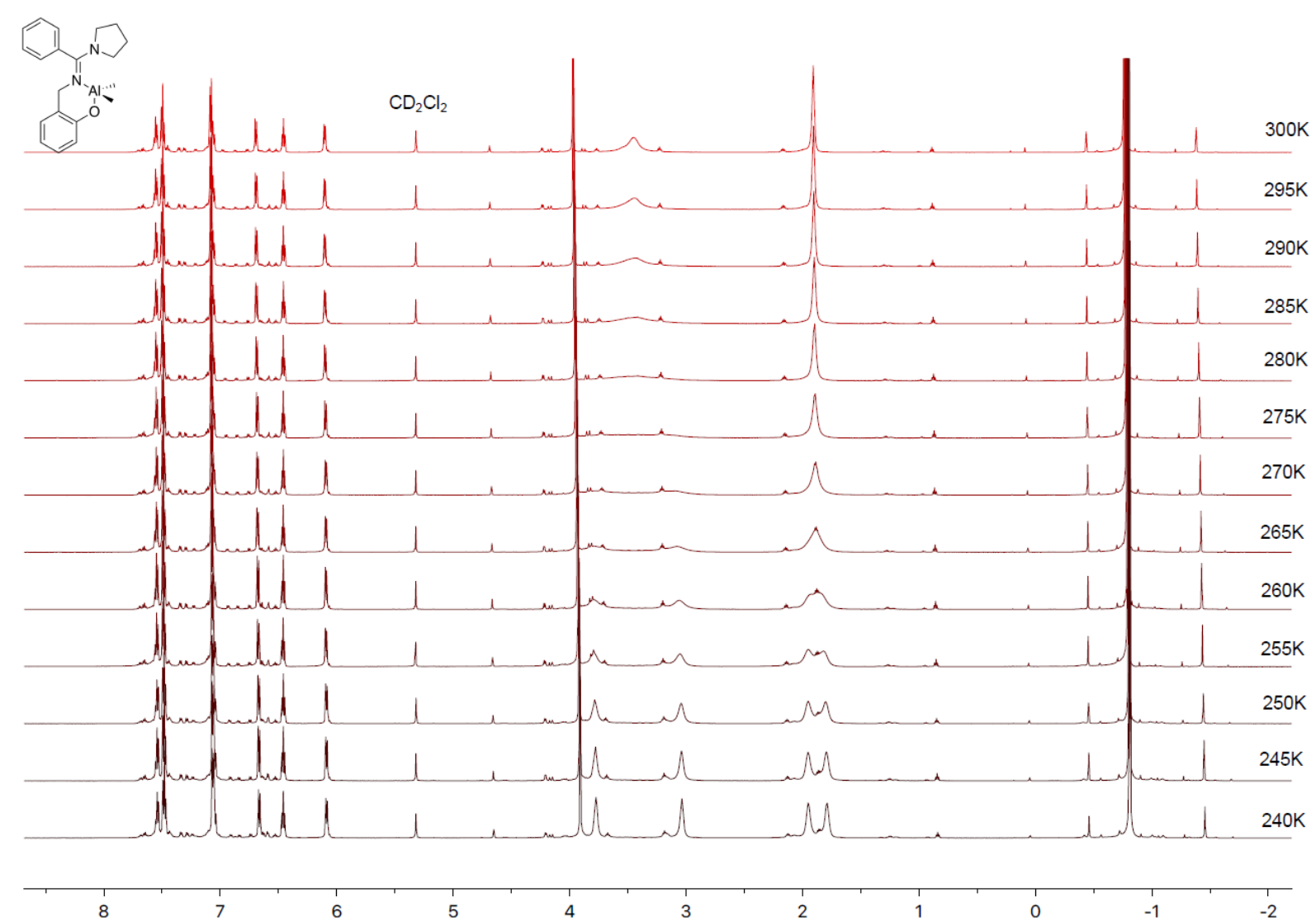

Figure S83: Variable Temperature ${ }^{1} \mathrm{H}$ NMR $\left(600 \mathrm{MHz}, \mathrm{CD}_{2} \mathrm{Cl}_{2}\right)$ of $\mathbf{2 b}$

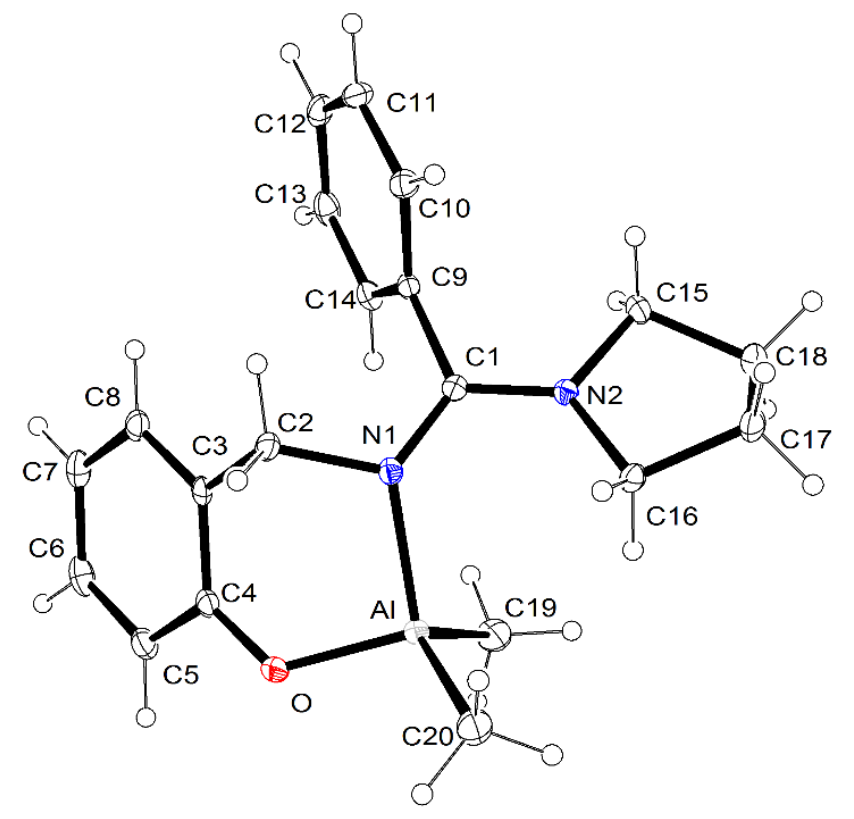

Figure S84: Crystal structure of $\mathbf{2 b}$ 
Table S9: Crystal data and structure refinement of 2b

\begin{tabular}{|c|c|}
\hline Identification code in cif & $\mathbf{C 2 b}$ \\
\hline Empirical formula & $\mathrm{C}_{20} \mathrm{H}_{25} \mathrm{AlN}_{2} \mathrm{O}$ \\
\hline Formula weight & 336.40 \\
\hline Temperature/K & 100 \\
\hline Crystal system & monoclinic \\
\hline Space group & $P 2_{1} / n$ \\
\hline $\mathrm{a} / \AA ̊$ & $10.3596(6)$ \\
\hline $\mathrm{b} / \AA$ & $18.3925(10)$ \\
\hline $\mathrm{c} / \AA$ & $10.4926(6)$ \\
\hline$\alpha /^{\circ}$ & 90 \\
\hline$\beta /{ }^{\circ}$ & $111.591(2)$ \\
\hline$\gamma /{ }^{\circ}$ & 90 \\
\hline Volume $/ \AA^{3}$ & 1858.97(18) \\
\hline $\mathrm{Z}$ & 4 \\
\hline$\rho_{\text {calc }} \mathrm{g} / \mathrm{cm}^{3}$ & 1.202 \\
\hline$\mu / \mathrm{mm}^{-1}$ & 0.118 \\
\hline $\mathrm{F}(000)$ & 720.0 \\
\hline Crystal size $/ \mathrm{mm}^{3}$ & $0.329 \times 0.279 \times 0.163$ \\
\hline Radiation & $\operatorname{MoK} \alpha(\lambda=0.71073)$ \\
\hline \multicolumn{2}{|c|}{$2 \Theta$ range for data collection $/{ }^{\circ} 6.952$ to 55.048} \\
\hline Index ranges & $-13 \leq \mathrm{h} \leq 13,-23 \leq \mathrm{k} \leq 23,-13 \leq 1 \leq 13$ \\
\hline Reflections collected & 32327 \\
\hline Independent reflections & $4271\left[\mathrm{R}_{\mathrm{int}}=0.0425, \mathrm{R}_{\text {sigma }}=0.0269\right]$ \\
\hline Data/restraints/parameters & $4271 / 0 / 219$ \\
\hline Goodness-of-fit on $\mathrm{F}^{2}$ & 1.065 \\
\hline Final $\mathrm{R}$ indexes $[\mathrm{I}>=2 \sigma(\mathrm{I})]$ & $\mathrm{R}_{1}=0.0446, \mathrm{wR}_{2}=0.1032$ \\
\hline Final $\mathrm{R}$ indexes [all data] & $\mathrm{R}_{1}=0.0577, \mathrm{wR}_{2}=0.1098$ \\
\hline \multicolumn{2}{|c|}{ Largest diff. peak/hole / e $\AA^{-3} 0.48 /-0.30$} \\
\hline $\mathrm{CCDC}$ & 1868750 \\
\hline
\end{tabular}




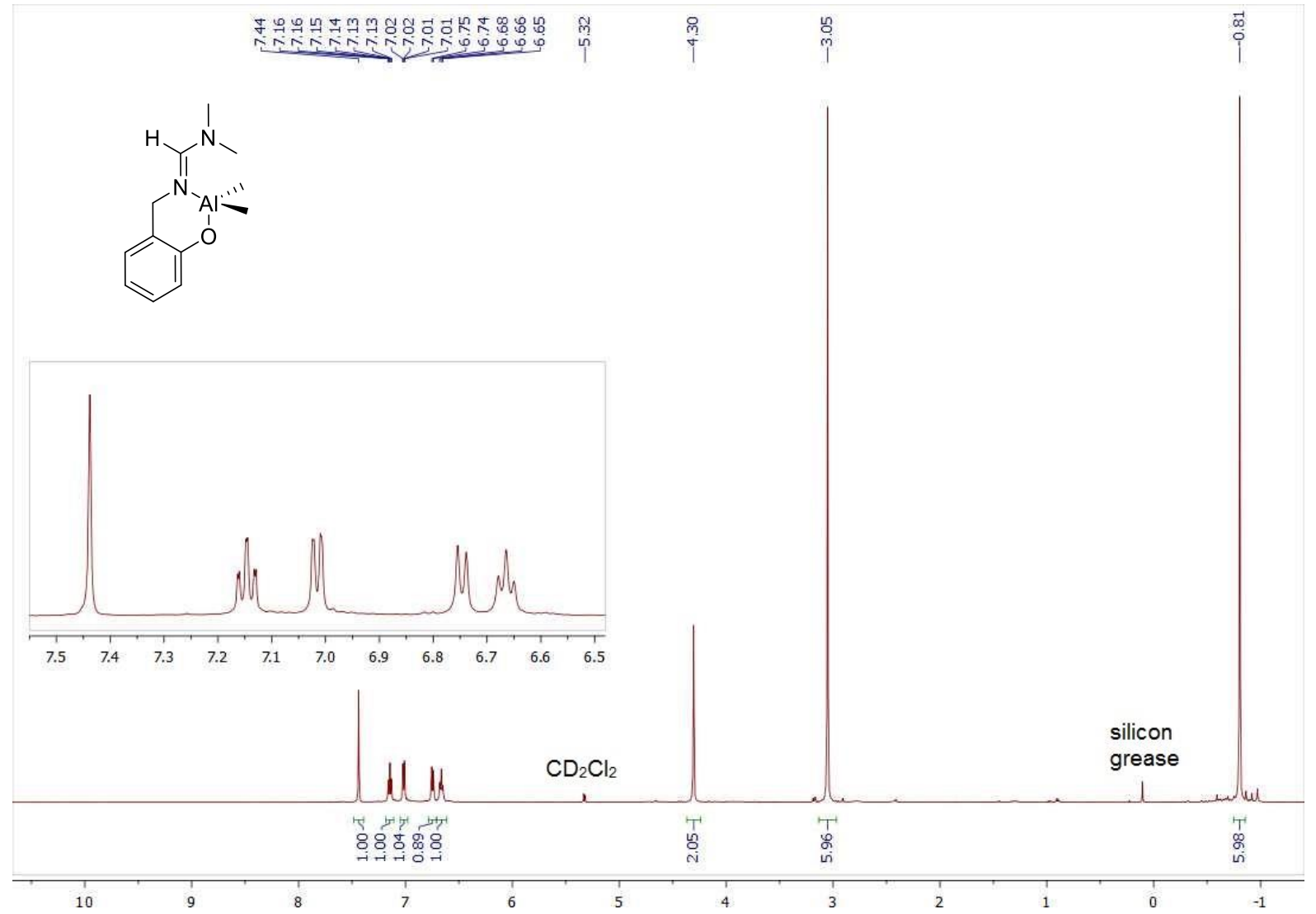

Figure S85: ${ }^{1} \mathrm{H} \mathrm{NMR}\left(500 \mathrm{MHz}, \mathrm{CD}_{2} \mathrm{Cl}_{2}\right)$ of $\mathbf{3 b}$

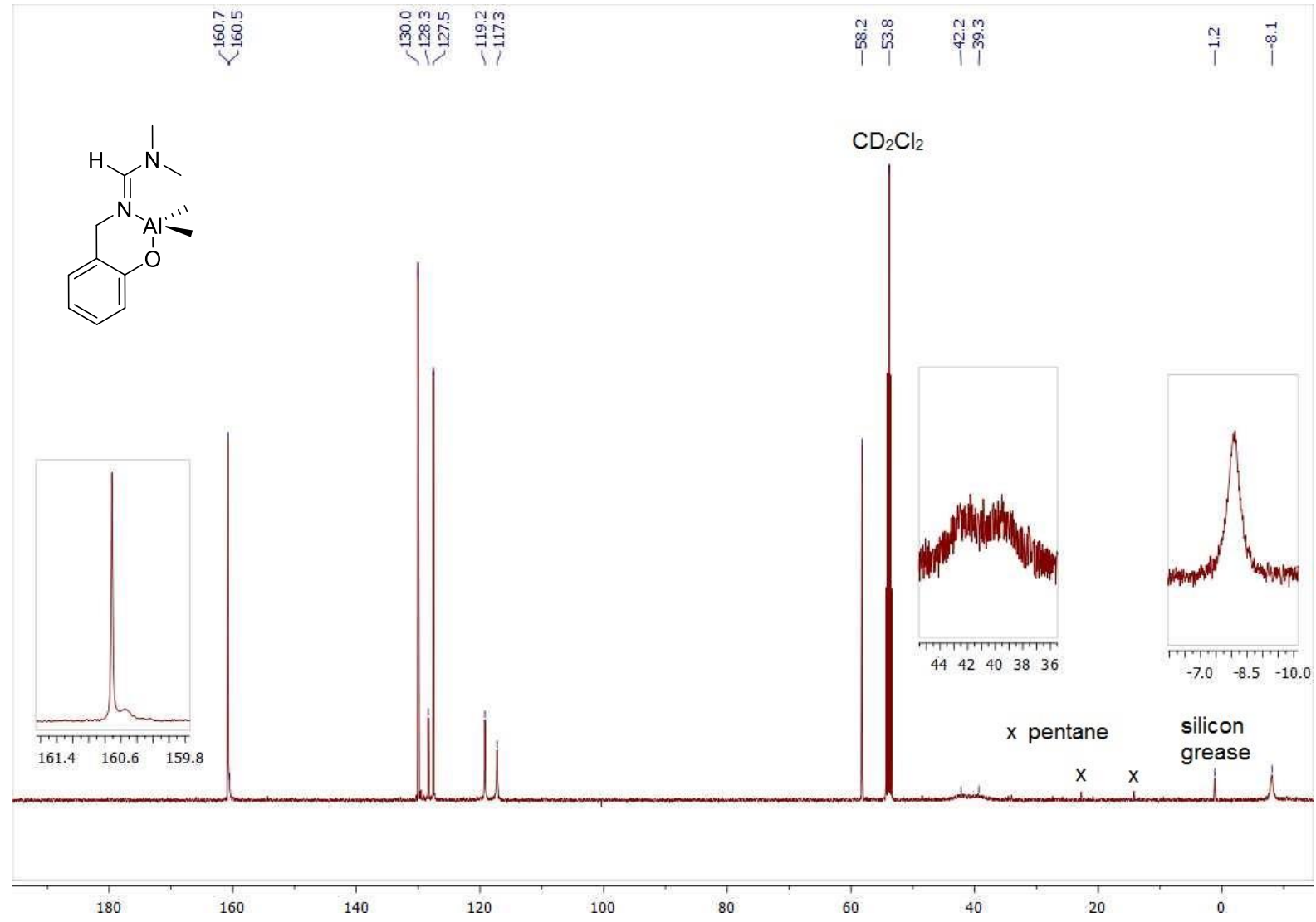

Figure S86: ${ }^{13} \mathrm{C}\left\{{ }^{1} \mathrm{H}\right\}$ NMR $\left(126 \mathrm{MHz}, \mathrm{CD}_{2} \mathrm{Cl}_{2}\right)$ of $\mathbf{3 b}$ 


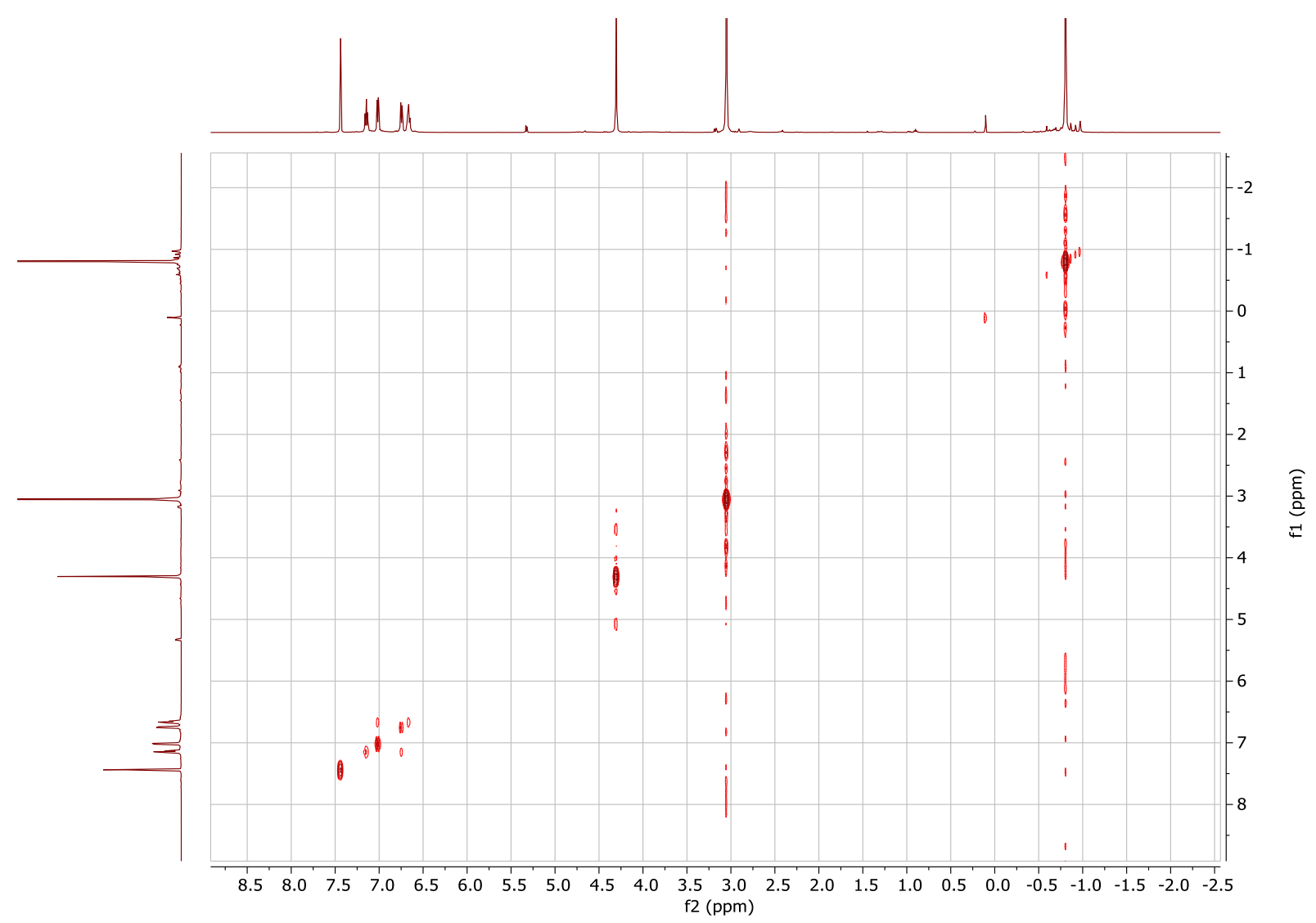

Figure S87: ${ }^{1} \mathrm{H}^{1} \mathrm{H}$ COSY (500 $\left.\mathrm{MHz}, \mathrm{CD}_{2} \mathrm{Cl}_{2}\right)$ of $\mathbf{3 b}$

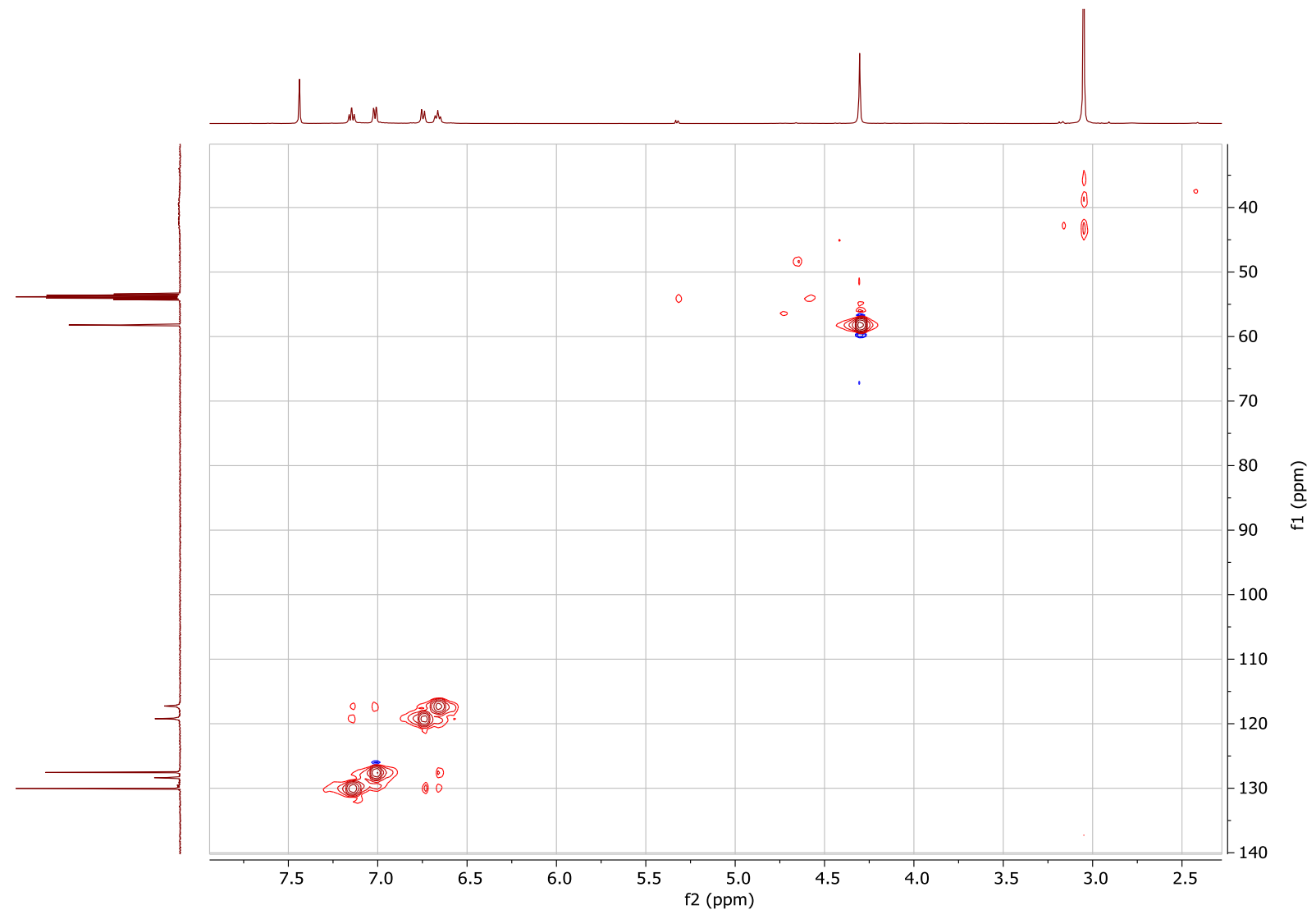

Figure S88: ${ }^{1} \mathrm{H}^{13} \mathrm{C} \mathrm{HSQC}\left(500 \mathrm{MHz} / 126 \mathrm{MHz}, \mathrm{CD}_{2} \mathrm{Cl}_{2}\right)$ of $\mathbf{3 b}$ 


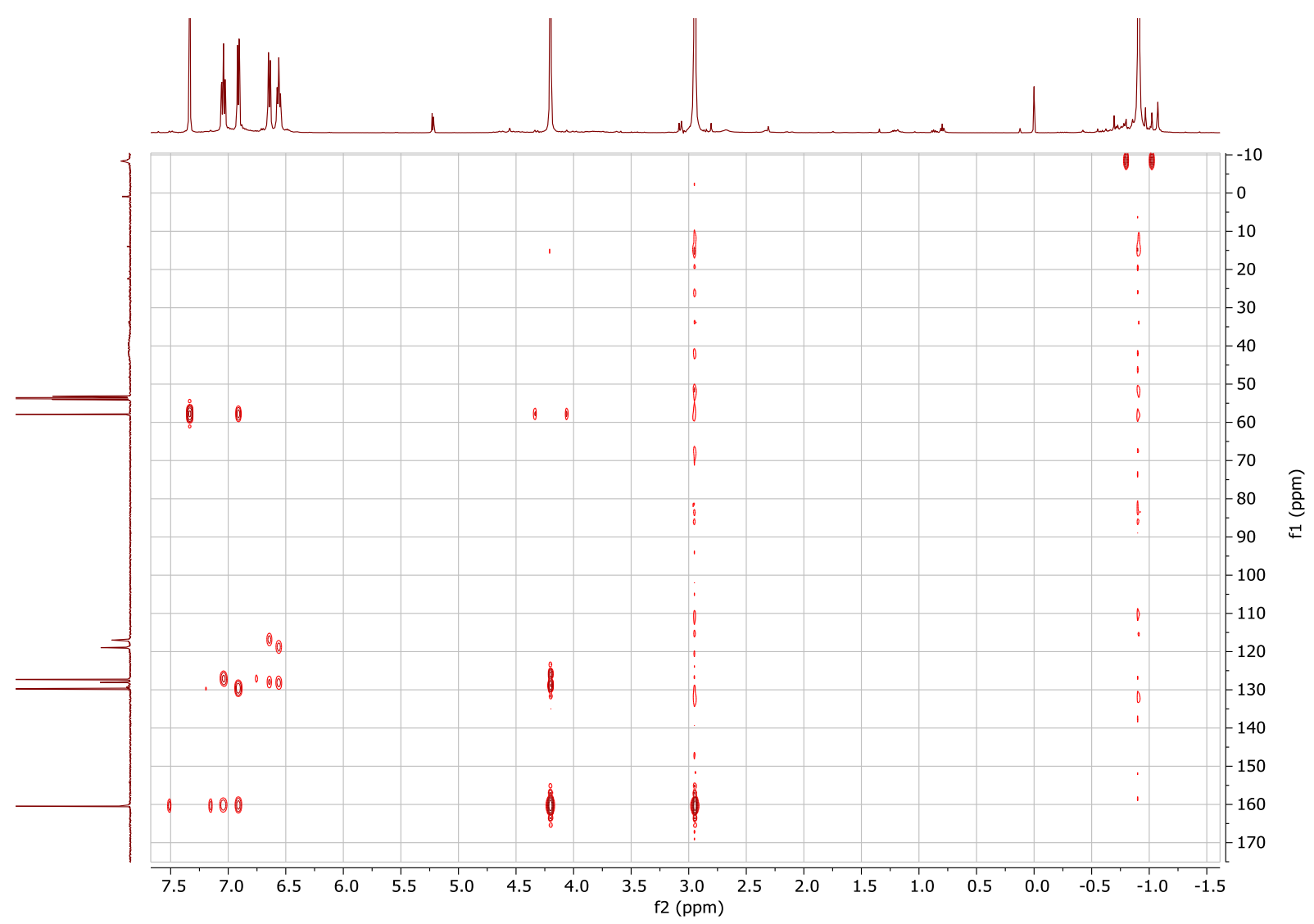

Figure S89: ${ }^{1} \mathrm{H}^{13} \mathrm{C} \mathrm{HMBC}\left(500 \mathrm{MHz} / 126 \mathrm{MHz}, \mathrm{CD}_{2} \mathrm{Cl}_{2}\right)$ of $\mathbf{3 b}$

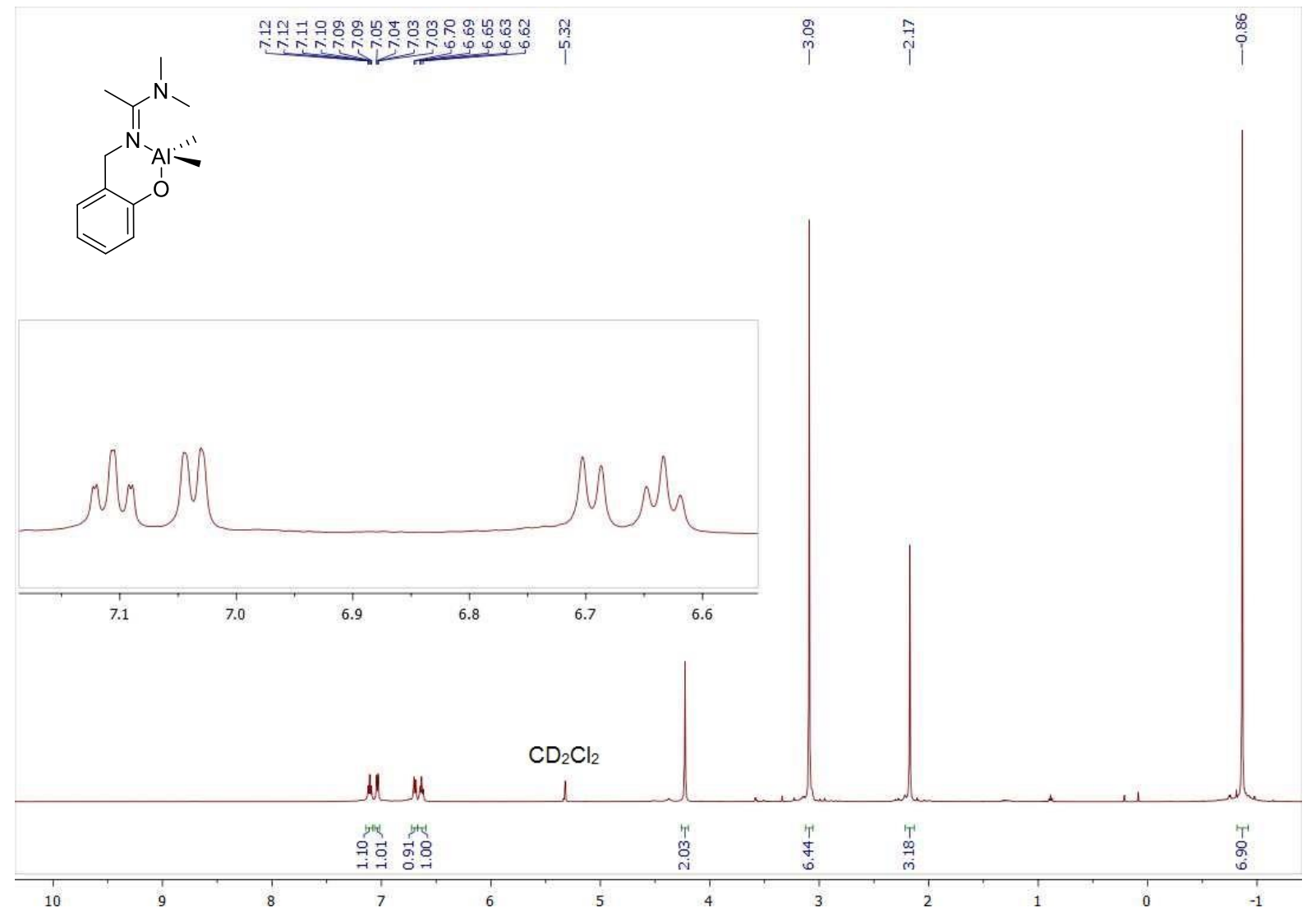

Figure S90: ${ }^{1} \mathrm{H}$ NMR $\left(500 \mathrm{MHz}, \mathrm{CD}_{2} \mathrm{Cl}_{2}\right)$ of $\mathbf{4 b}$ 


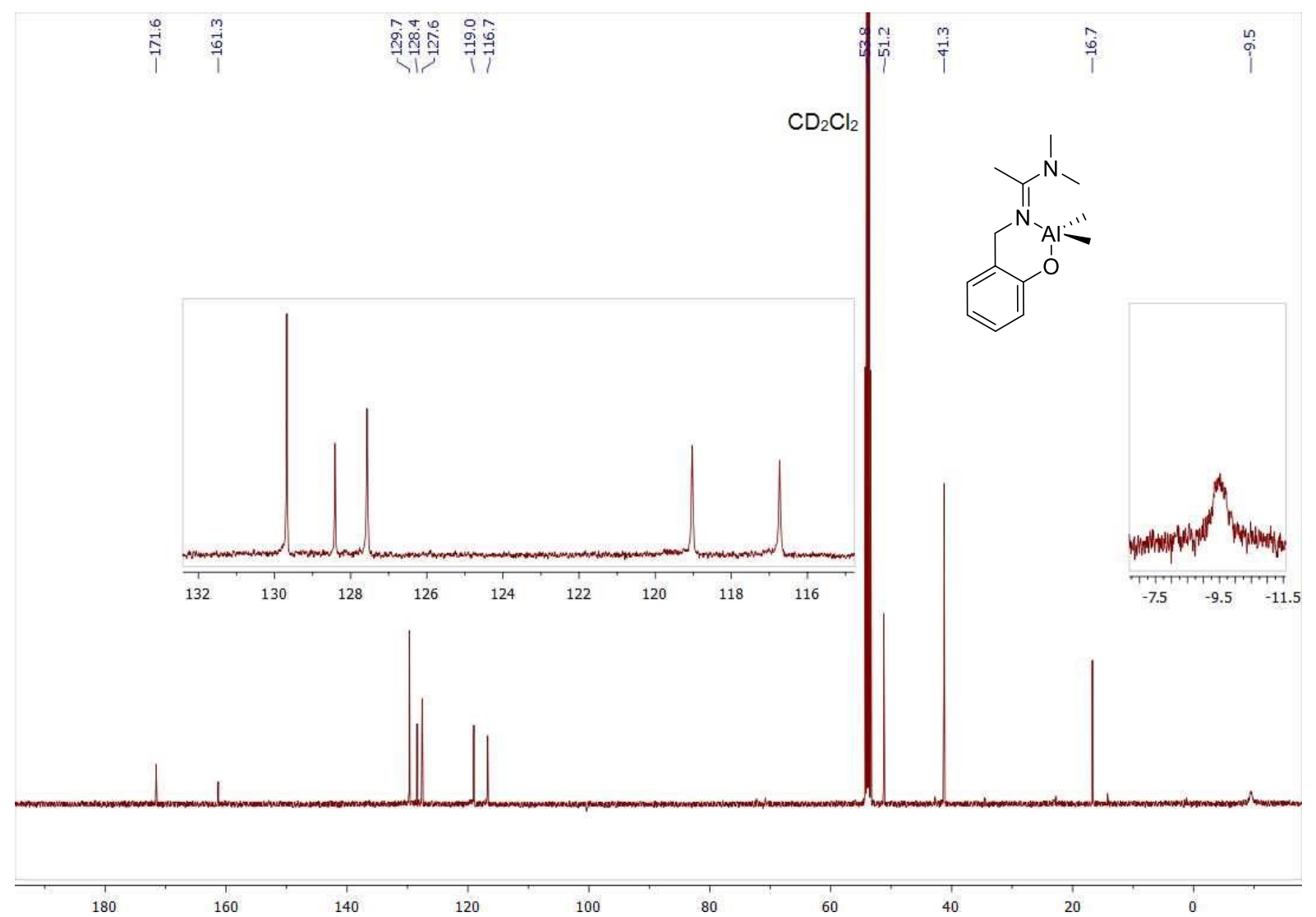

Figure S91: ${ }^{13} \mathrm{C}\left\{{ }^{1} \mathrm{H}\right\}$ NMR $\left(126 \mathrm{MHz}, \mathrm{CD}_{2} \mathrm{Cl}_{2}\right)$ of $\mathbf{4 b}$

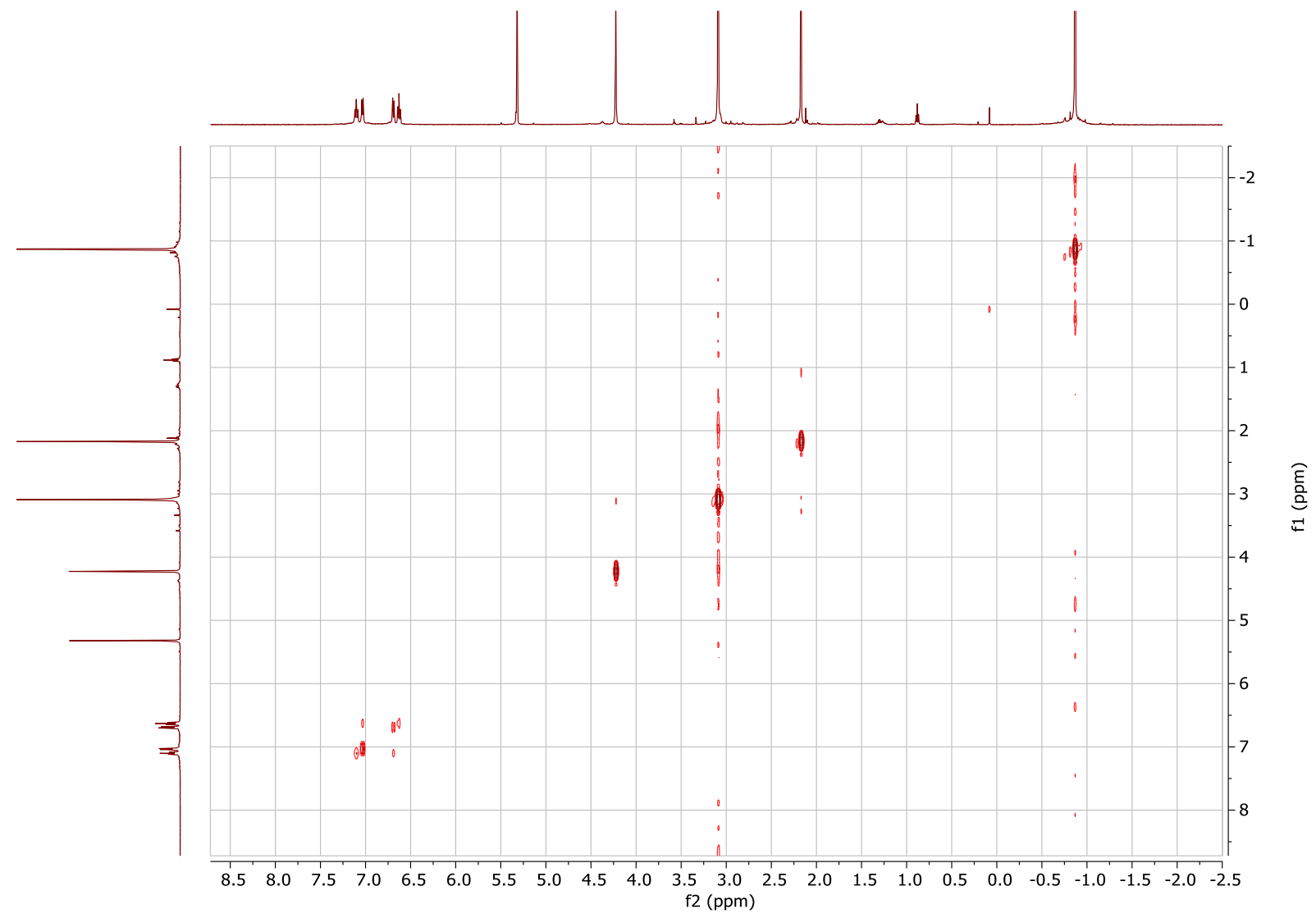

Figure S92: ${ }^{1} \mathrm{H}^{1} \mathrm{H} \operatorname{COSY}\left(500 \mathrm{MHz}, \mathrm{CD}_{2} \mathrm{Cl}_{2}\right)$ of $\mathbf{4 b}$ 


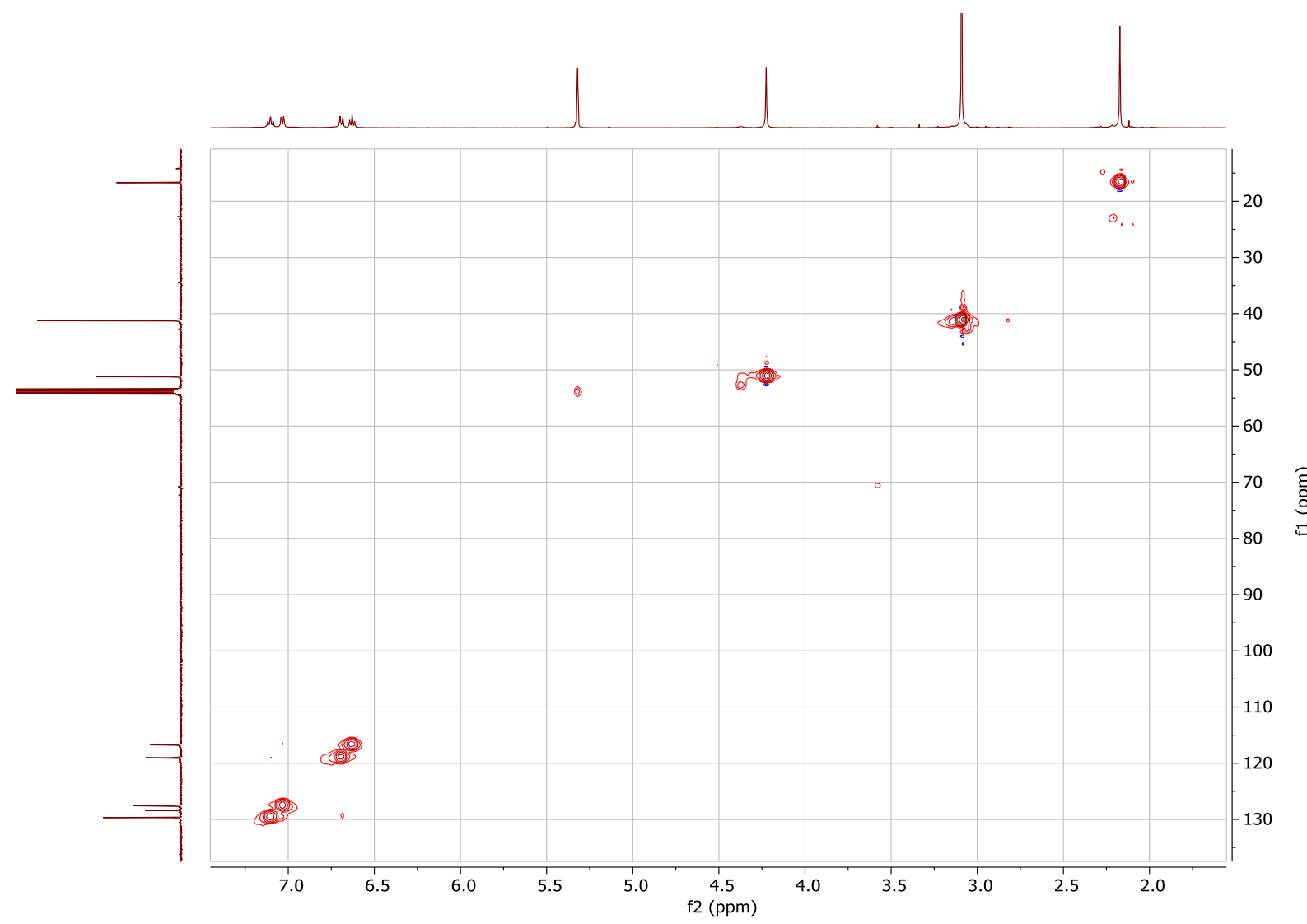

Figure S93: ${ }^{1} \mathrm{H}^{13} \mathrm{C}$ HSQC $\left(500 \mathrm{MHz} / 126 \mathrm{MHz}, \mathrm{CD}_{2} \mathrm{Cl}_{2}\right)$ of $\mathbf{4 b}$

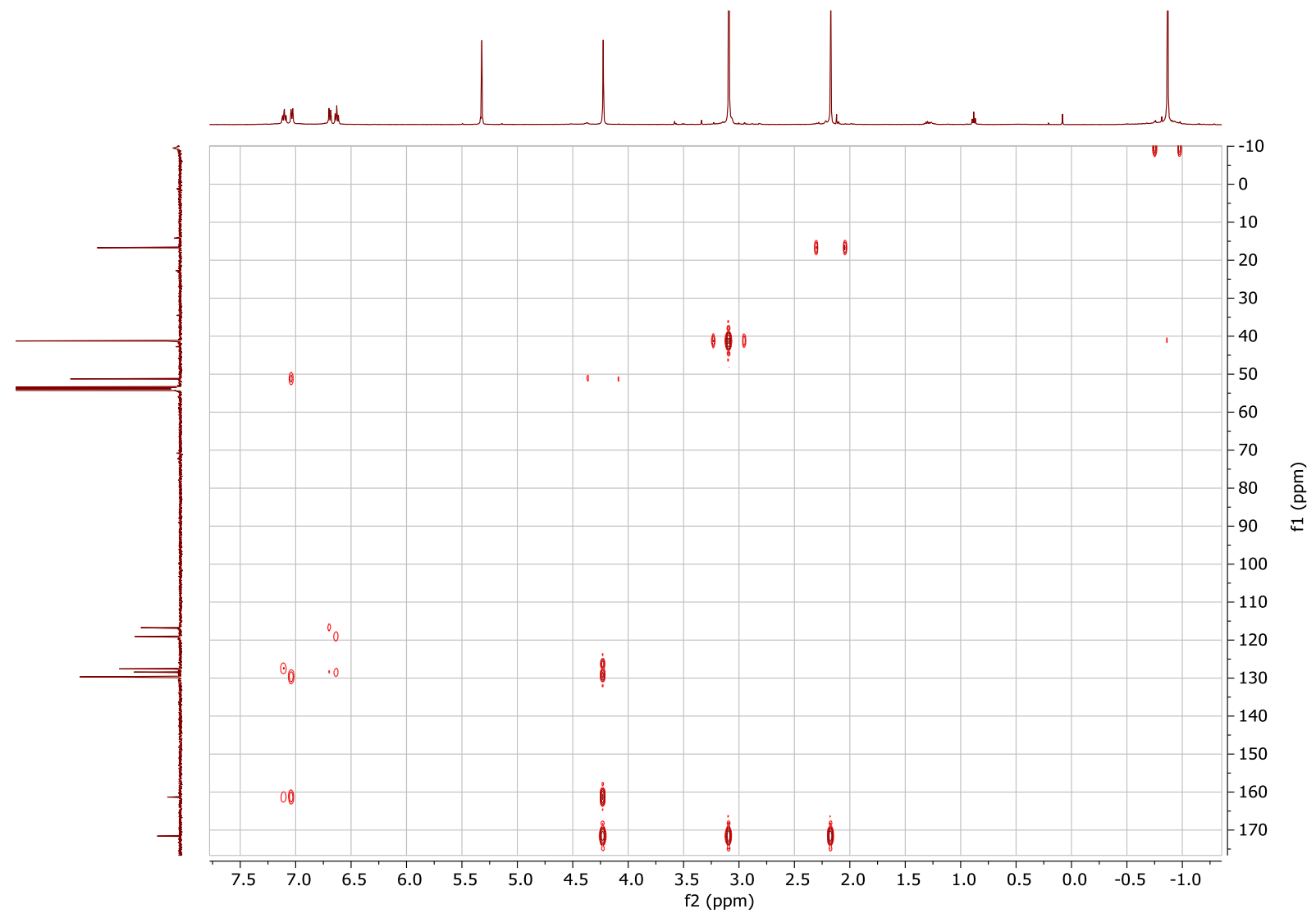

Figure S94: ${ }^{1} \mathrm{H}^{13} \mathrm{C} \mathrm{HMBC}\left(500 \mathrm{MHz} / 126 \mathrm{MHz}, \mathrm{CD}_{2} \mathrm{Cl}_{2}\right)$ of $\mathbf{4 b}$ 


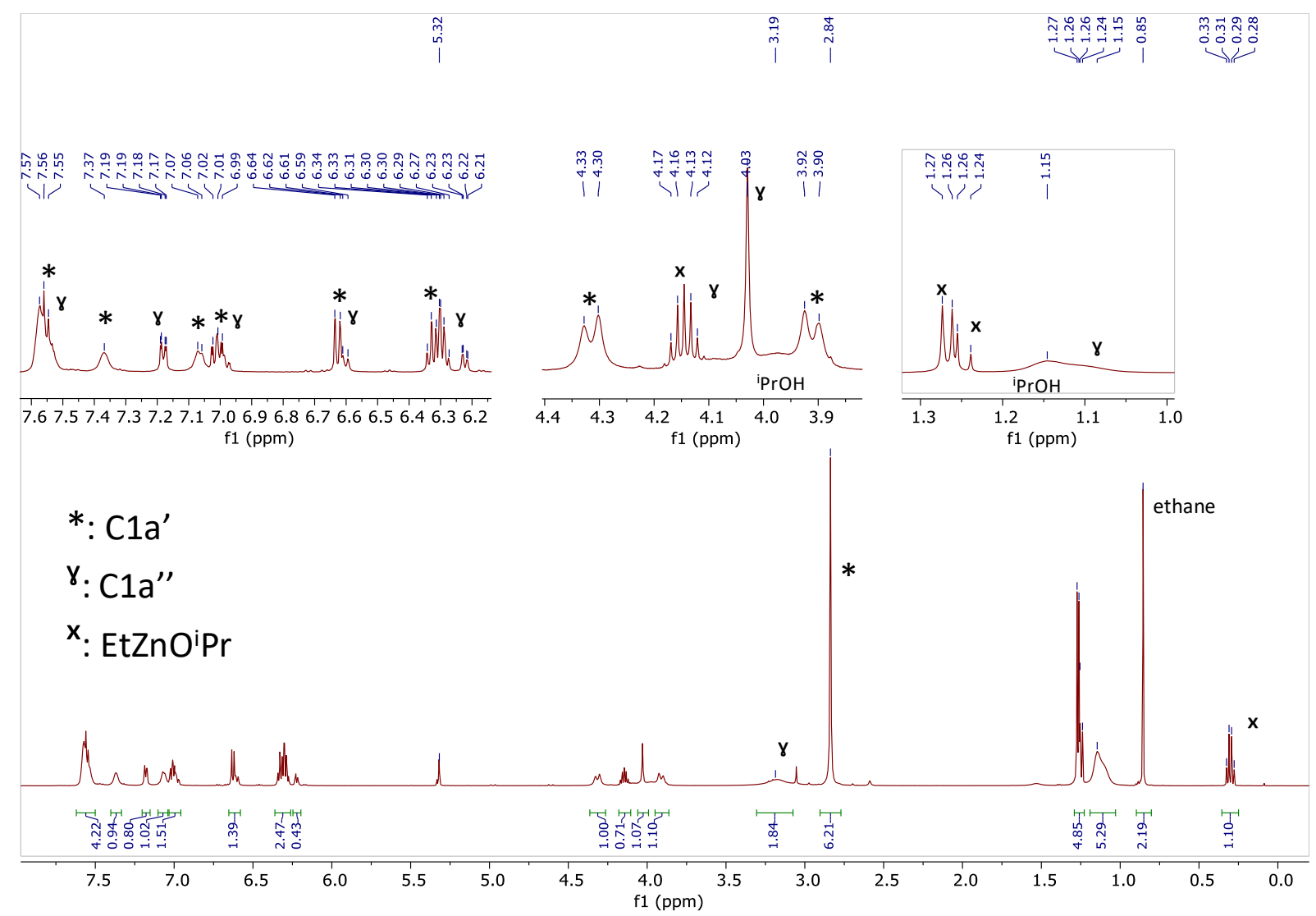

Figure S95: ${ }^{1} \mathrm{H}$ NMR $\left(500 \mathrm{MHz}, \mathrm{CD}_{2} \mathrm{Cl}_{2}\right)$ of a 1/1 1a/ $/{ }^{i} \mathrm{PrOH}$ mixture in $\mathrm{CD}_{2} \mathrm{Cl}_{2}$ after $2 \mathrm{~h}$ at room temperature.

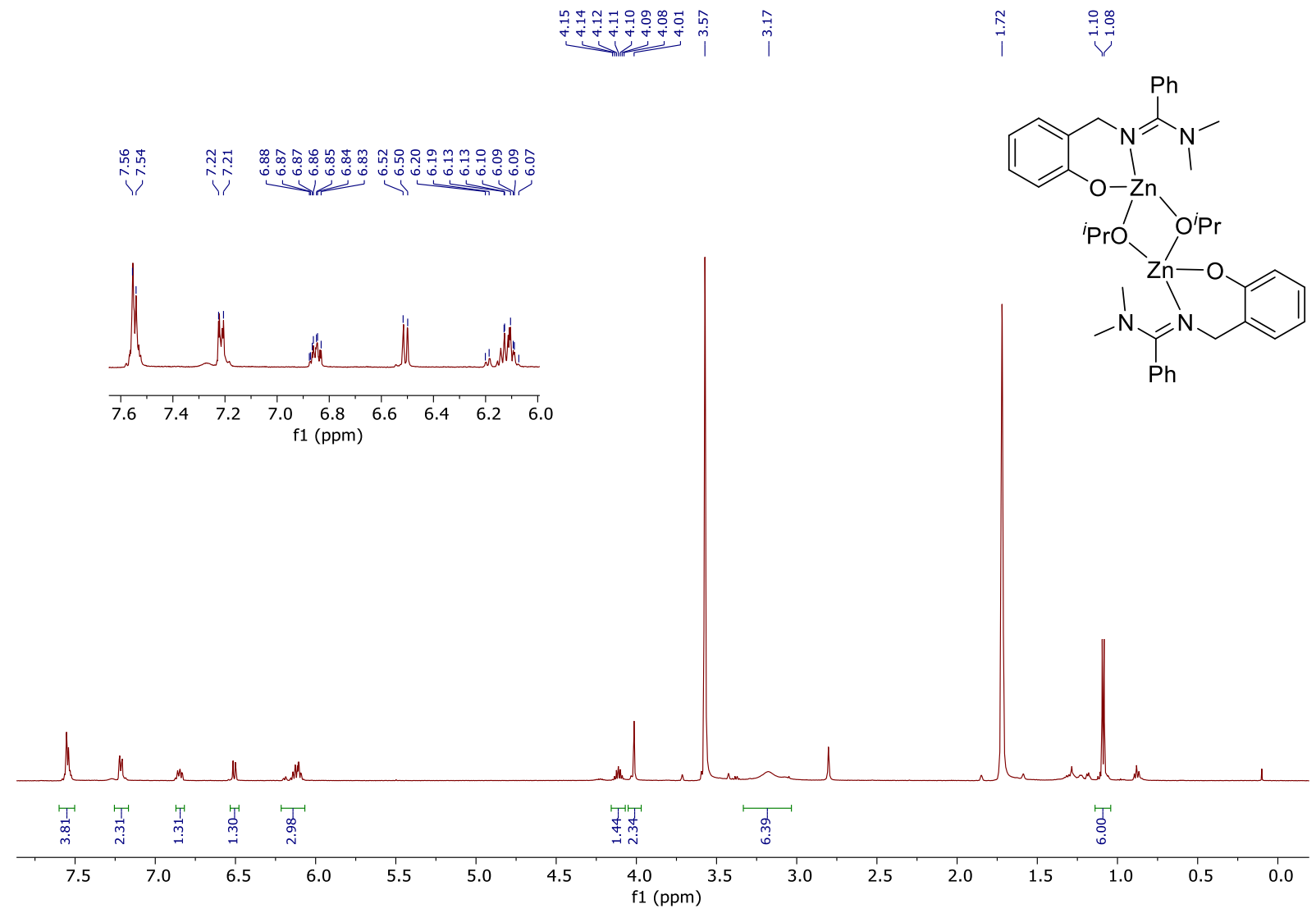

Figure S96: ${ }^{1} \mathrm{H}$ NMR $(500 \mathrm{MHz}, \mathrm{THF}-\mathrm{d} 8)$ of crystals of 1a” dissolved in THF-d8 


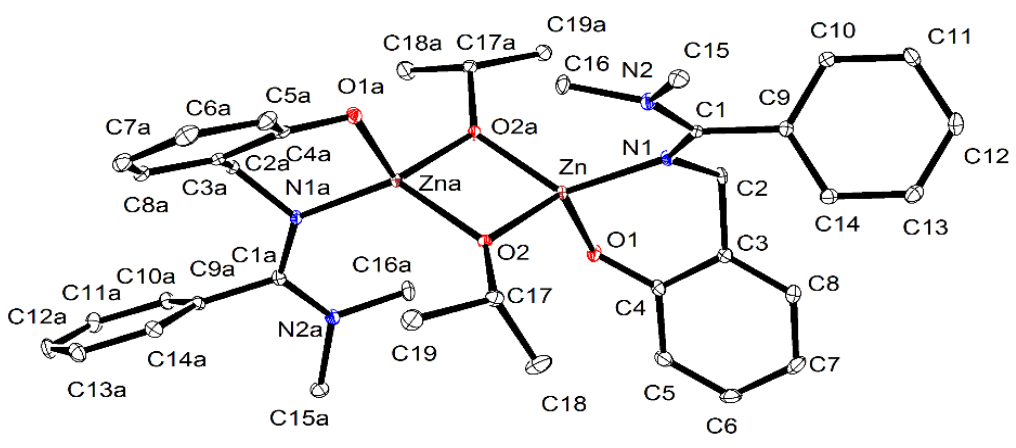

Figure S97: Crystal structure of 1a"

Table S10: Crystal data and structure refinement of 1a"

\begin{tabular}{|c|c|}
\hline Identification code in cif & C1a”, \\
\hline Formula & $\mathrm{C}_{38} \mathrm{H}_{48} \mathrm{~N}_{4} \mathrm{O}_{4} \mathrm{Zn}_{2}$ \\
\hline$D_{\text {calc. }} / \mathrm{g} \mathrm{cm}^{-3}$ & 1.364 \\
\hline $\mathrm{m} / \mathrm{mm}^{-1}$ & 1.347 \\
\hline Formula Weight & 755.54 \\
\hline Color & clear light colorle \\
\hline Shape & block \\
\hline Size $/ \mathrm{mm}^{3}$ & $0.56 \times 0.37 \times 0.32$ \\
\hline$T / \mathrm{K}$ & $100.0(1)$ \\
\hline Crystal System & triclinic \\
\hline Space Group & $P-1$ \\
\hline$a / \AA$ & $9.8579(6)$ \\
\hline$b / \AA$ & $13.0687(9)$ \\
\hline$c / \AA$ & $14.9319(9)$ \\
\hline$a l^{\circ}$ & $104.572(2)$ \\
\hline$b 1^{\circ}$ & $95.036(2)$ \\
\hline$g l^{\circ}$ & $95.720(2)$ \\
\hline$V / \AA^{3}$ & $1839.8(2)$ \\
\hline$Z$ & 2 \\
\hline$Z^{\prime}$ & 1 \\
\hline Wavelength/Å & 0.710760 \\
\hline Radiation type & $\operatorname{MoK}_{a}$ \\
\hline$Q_{\min } l^{\circ}$ & 3.184 \\
\hline$Q_{\max } I^{\circ}$ & 27.585 \\
\hline Measured Refl. & 162673 \\
\hline Independent Refl. & 8495 \\
\hline Reflections with $\mathrm{I}>2(\mathrm{I})$ & 7511 \\
\hline$R_{\text {int }}$ & 0.0329 \\
\hline Parameters & 441 \\
\hline Restraints & 0 \\
\hline Largest Peak & 0.692 \\
\hline Deepest Hole & -0.441 \\
\hline GooF & 1.158 \\
\hline$w R_{2}$ (all data) & 0.0629 \\
\hline$w R_{2}$ & 0.0580 \\
\hline$R_{I}$ (all data) & 0.0344 \\
\hline$R_{1}$ & 0.0266 \\
\hline CCDC & 1937786 \\
\hline
\end{tabular}




\section{Catalyst 1a}

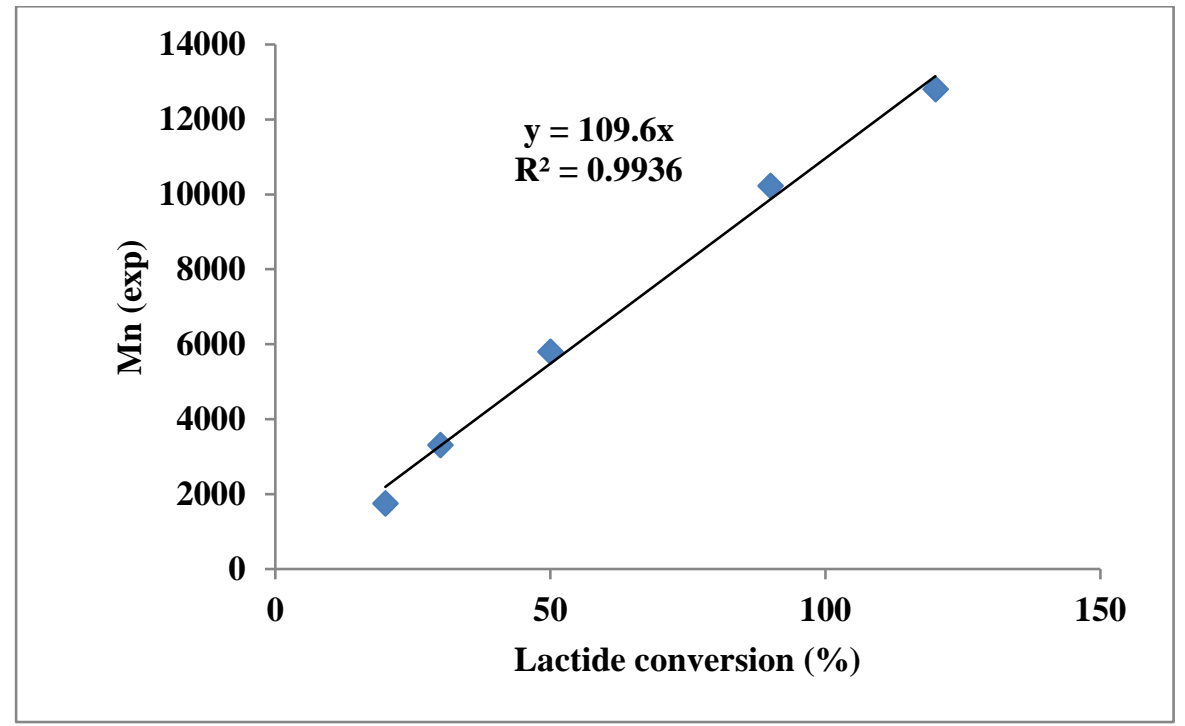

Figure S98. Graph of the PLA $M_{\mathrm{n}}$ values as a function of lactide conversion for the ROP of rac-lactide initiated by the 1a/ ${ }^{i} \mathrm{PrOH}$ system. Conditions: 100 equiv. of rac-lactide (vs. 1a), 1 equiv. of ${ }^{i} \mathrm{PrOH}$ (vs. 1a), $[\text { rac-lactide }]_{0}=1 \mathrm{M}, \mathrm{CH}_{2} \mathrm{Cl}_{2}, \mathrm{RT}_{2} 2 \mathrm{~h}$.

\section{Catalyst 2a}

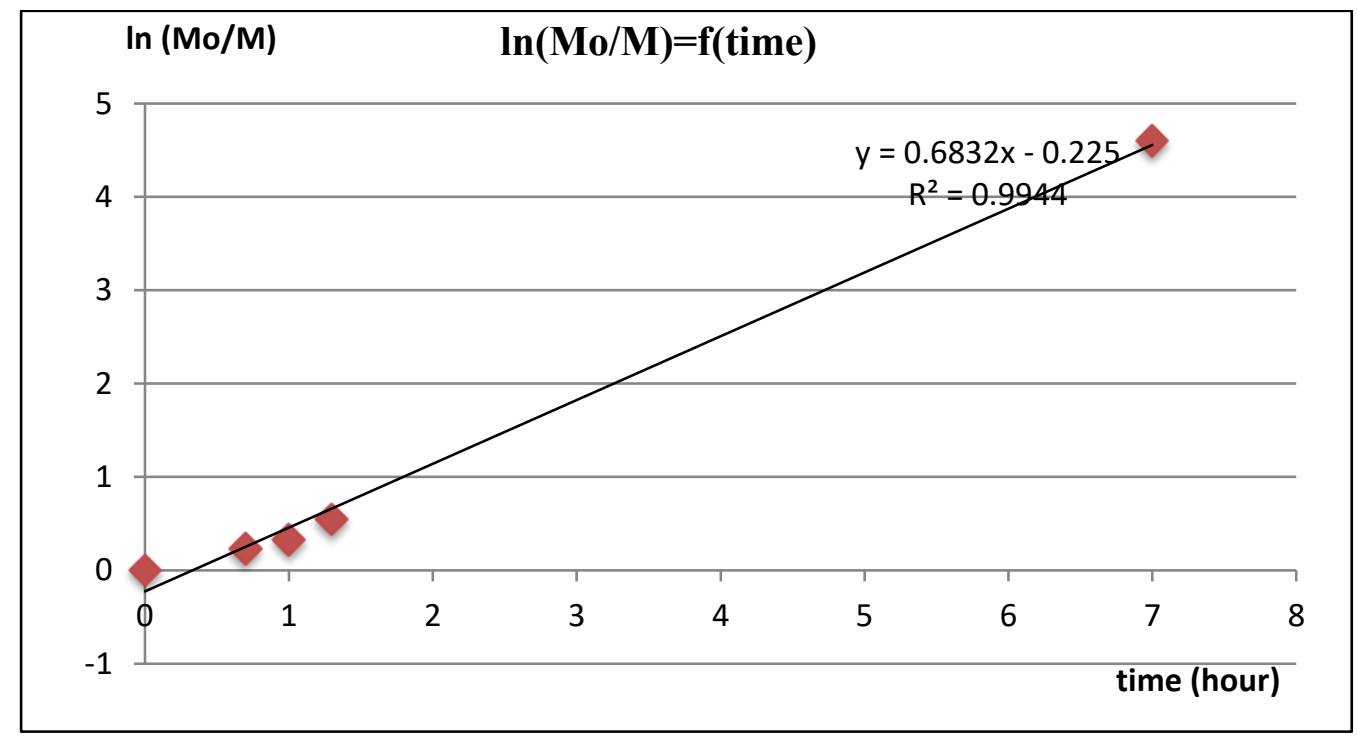

Figure S99. Graph of $\ln ([\mathrm{M}] 0 /[\mathrm{M}])$ versus time for the ROP of rac-lactide initiated by the $\mathbf{2 a} / \mathrm{BnOH}$ system. Conditions: 100 equiv. of rac-lactide (vs. 2a), 1 equiv. of $\mathrm{BnOH}$ (vs. 2a), $[\mathrm{M}]_{0}=[\text { rac-lactide }]_{0}=1 \mathrm{M}, \mathrm{CH}_{2} \mathrm{Cl}_{2}, \mathrm{RT}, 7 \mathrm{~h}$. 


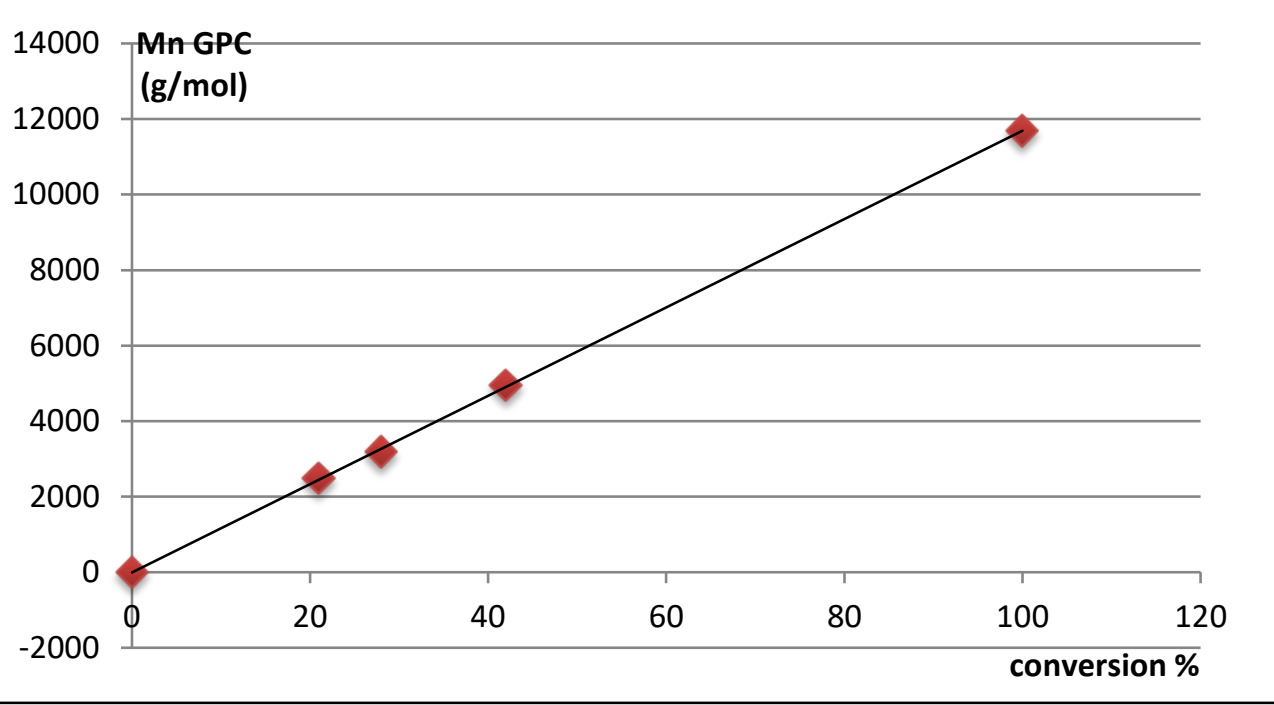

Figure S100. Graph of the PLA $M_{\mathrm{n}}$ values as a function of lactide conversion for the ROP of $r a c$-lactide initiated by the 2a/ $\mathrm{BnOH}$ system. Conditions: 100 equiv. of rac-lactide (vs. 2a), 1 equiv. of $\mathrm{BnOH}(v s . \mathbf{2 a}),[\mathrm{M}]_{0}=[\mathrm{rac} \text {-lactide }]_{0}=1 \mathrm{M}, \mathrm{CH}_{2} \mathrm{Cl}_{2}$, RT, 7 h.

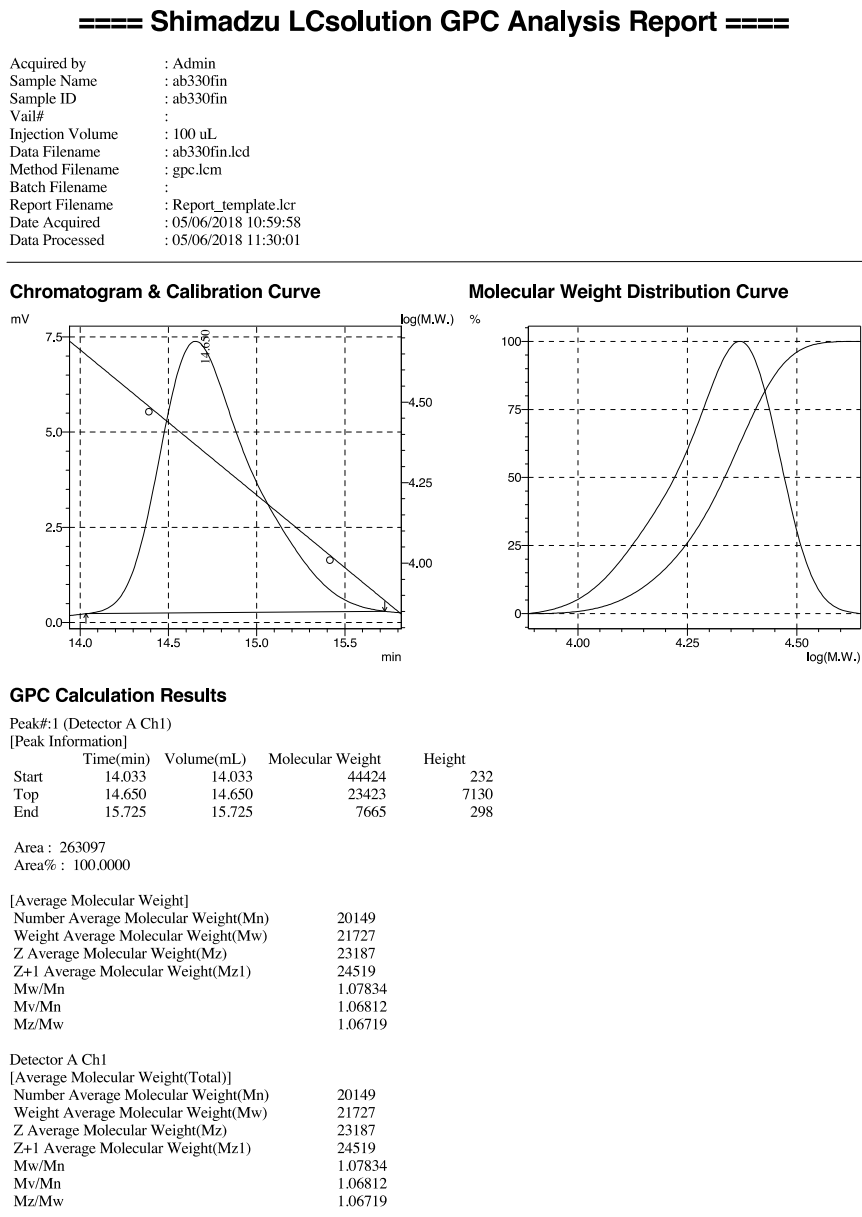

Figure S101. SEC traces of isolated PLA prepared via ROP of rac-lactide initiated by the 2a/BnOH system. (Conditions: 100 equiv. of rac-lactide (vs. 2a), 1 equiv. of $\mathrm{BnOH}$ (vs 2a), $[\text { rac-lactide }]_{0}=1 \mathrm{M}, \mathrm{CH}_{2} \mathrm{Cl}_{2}, \mathrm{RT}, 7 \mathrm{~h}$ ). 

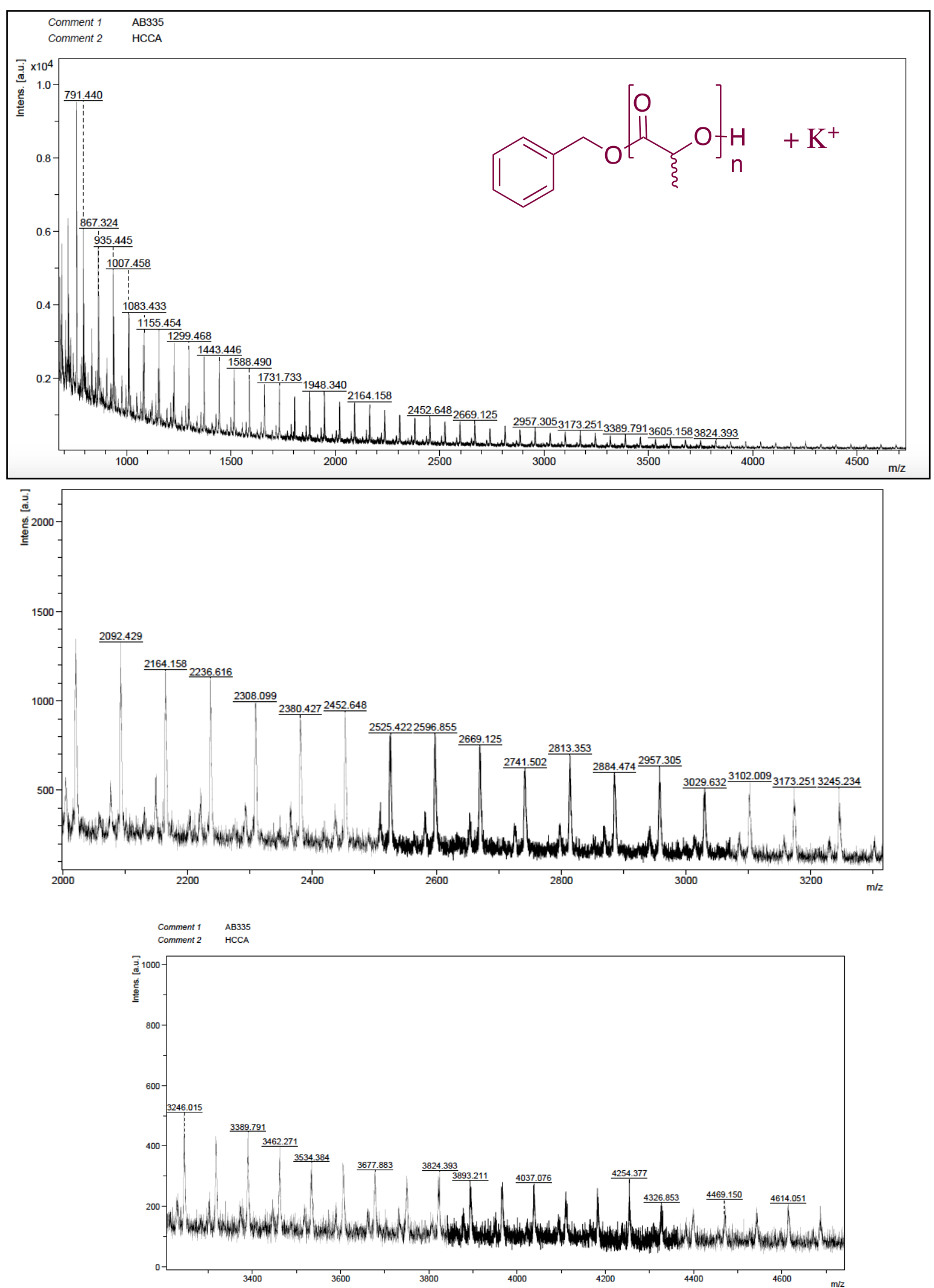

Figure S102. MALDI-TOF spectra of an isolated PLA oligomer prepared via ROP of rac-lactide initiated by the $\mathbf{2 a} / \mathrm{BnOH}$ system. (Conditions: 20 equiv. of $r a c$-lactide (vs. 2a), 1 equiv. of $\mathrm{BnOH}$ (vs. 2a), $[r a c \text {-lactide }]_{0}=1 \mathrm{M}, \mathrm{CH}_{2} \mathrm{Cl}_{2}, \mathrm{RT}, 1 \mathrm{~h}$ ). 


\section{Catalyst 3a}

$====$ Shimadzu LCsolution GPC Analysis Report ====

$\begin{array}{ll}\text { Acquired by } & : \text { Admin } \\ \text { Sample Name } & : \text { rl239 } \\ \text { Sample ID } & : \text { rl239 } \\ \text { Vail\# } & : \\ \text { Injection Volume } & : 100 \mathrm{uL} \\ \text { Data Filename } & : \text { rl 239.lcd } \\ \text { Method Filename } & : \text { gpc.lcm } \\ \text { Batch Filename } & : \\ \text { Report Filename } & : \text { Report_template.lcr } \\ \text { Date Acquired } & : 22 / 06 / 201713: 34: 36 \\ \text { Data Processed } & : 22 / 06 / 201714: 04: 37\end{array}$

\section{Chromatogram \& Calibration Curve}

$\mathrm{mV}$

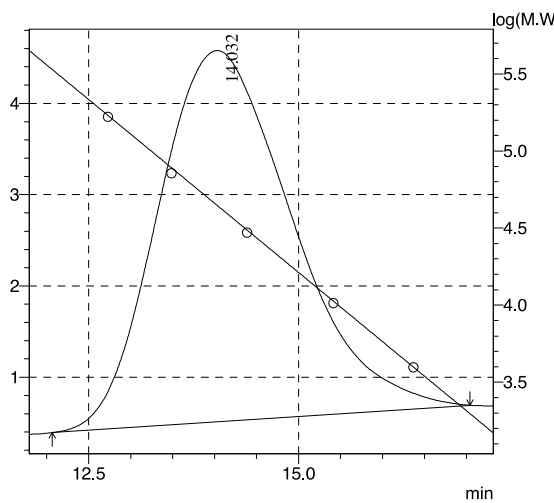

Molecular Weight Distribution Curve

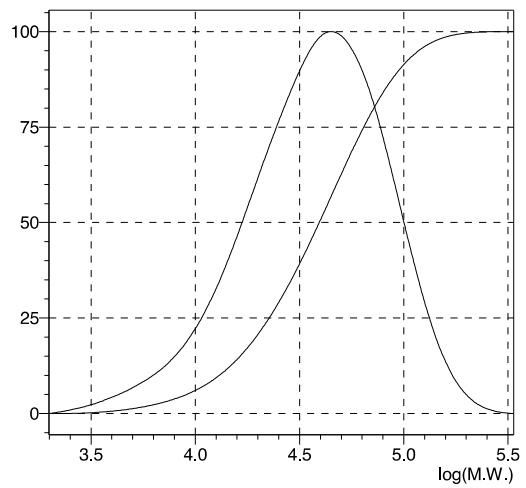

GPC Calculation Results

Peak\#: 1 (Detector A Ch1)

[Peak Information]

\begin{tabular}{lrrrr} 
& Time $(\mathrm{min})$ & Volume $(\mathrm{mL})$ & Molecular Weight & \multicolumn{1}{c}{ Height } \\
Start & 12.067 & 12.067 & 337453 & 392 \\
Top & 14.032 & 14.032 & 44312 & 4069 \\
End & 17.042 & 17.042 & 1977 & 691
\end{tabular}

Area : 450569

Area\% : 100.0000

[Average Molecular Weight]

Number Average Molecular Weight(Mn) 26337

$\begin{array}{ll}\text { Weight Average Molecular Weight(Mw) } & 48449 \\ \text { Z Average Molecular Weight(Mz) } & 74774\end{array}$

Z+1 Average Molecular Weight(Mz1)

$\mathrm{Mw} / \mathrm{Mn}$

$\mathrm{Mv} / \mathrm{Mn}$

1.83959

1.71811

Detector A Ch1

[Average Molecular Weight(Total)]

Number Average Molecular Weight(Mn) 26337

Weight Average Molecular Weight(Mw) $\quad 48449$

Z Average Molecular Weight(Mz) $\quad 74774$

Z+1 Average Molecular Weight(Mz1) 102786

$\mathrm{Mw} / \mathrm{Mn} \quad 1.83959$

$\mathrm{Mv} / \mathrm{Mn} \quad 1.71811$

Figure S103. SEC traces of isolated PLA prepared via ROP of $r a c$-lactide initiated by the $\mathbf{3 a} /{ }^{i} \mathrm{PrOH}$ system. Conditions: 100 equiv. of $r a c$-lactide (vs. 3a), 1 equiv. of ${ }^{i} \mathrm{PrOH},[\text { rac-lactide }]_{0}=1 \mathrm{M}$, dichloromethane, room temperature, $2 \mathrm{~h}$ : $96 \%$ conversion to PLA. 


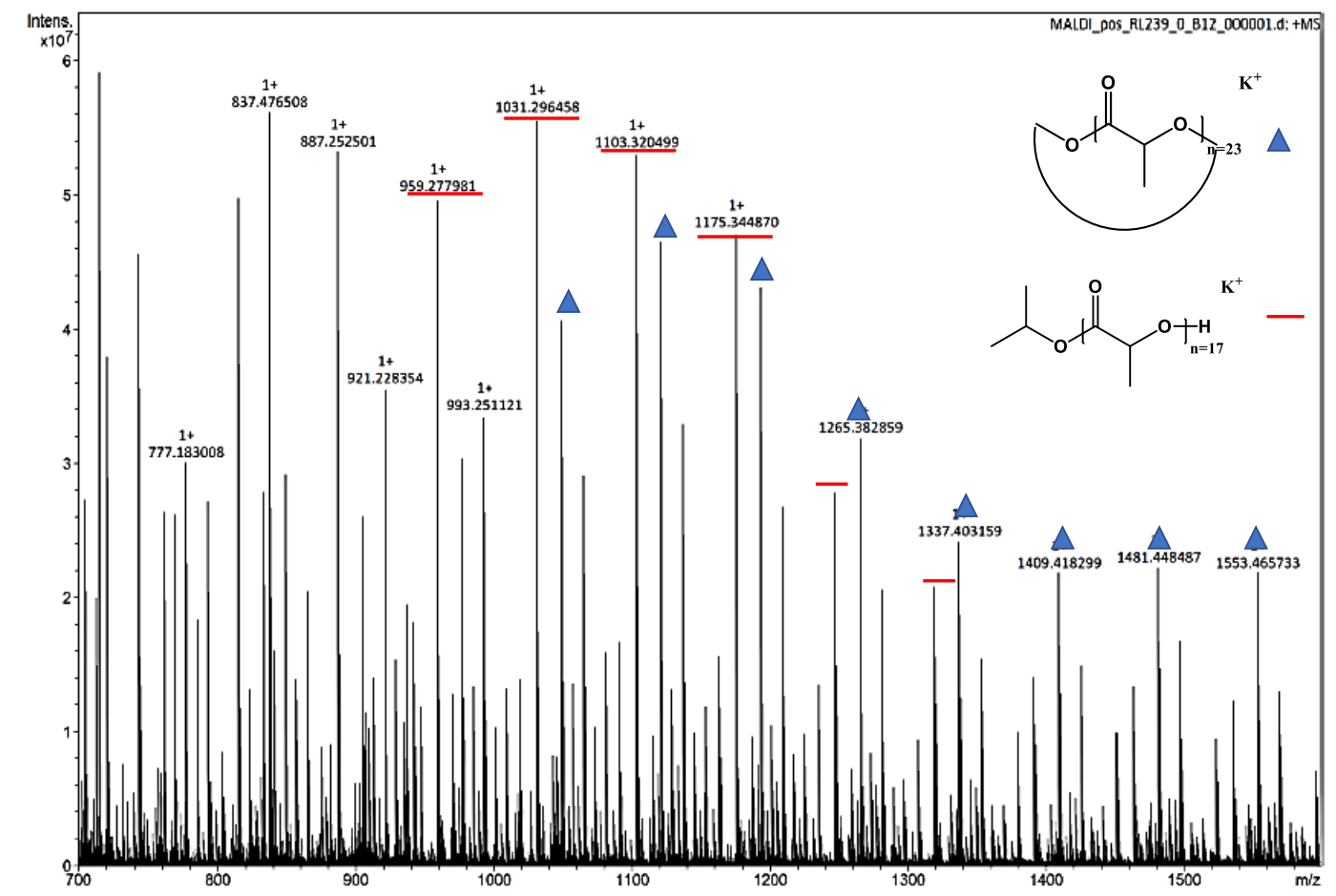

Figure S104. MALDI-TOF spectra of isolated PLA prepared via ROP of rac-lactide initiated by the 3a/ ${ }^{i} \mathrm{PrOH}$ system. (Conditions: 20 equiv. of $r a c$-lactide (vs. 3a), 1 equiv. of ${ }^{i} \mathrm{PrOH}$ (vs. 3a), $[\text { rac-lactide }]_{0}=1 \mathrm{M}, \mathrm{CH}_{2} \mathrm{Cl}_{2}, \mathrm{RT}, 2 \mathrm{~h}$ ). 


\section{Catalyst $4 \mathbf{a}$}

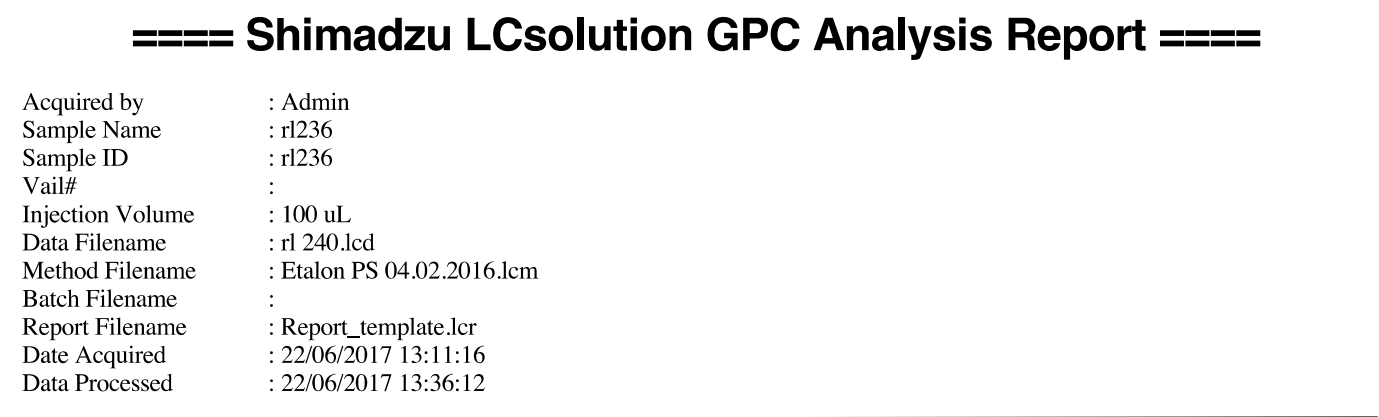

\section{Chromatogram \& Calibration Curve}

$\mathrm{mV}$

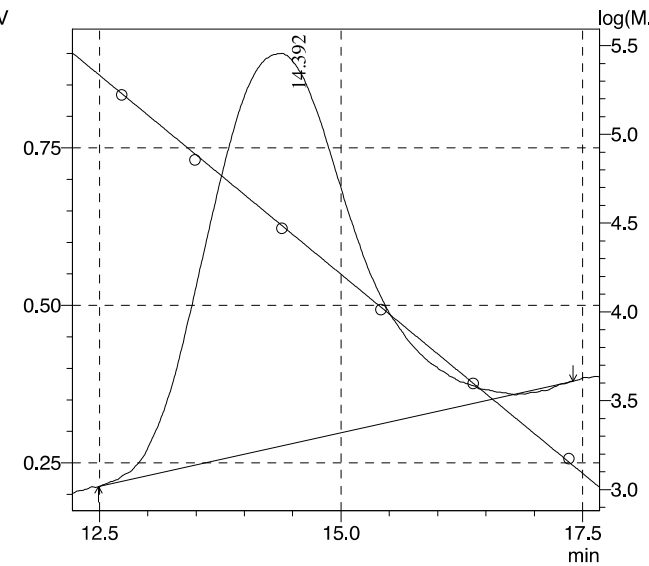

Molecular Weight Distribution Curve

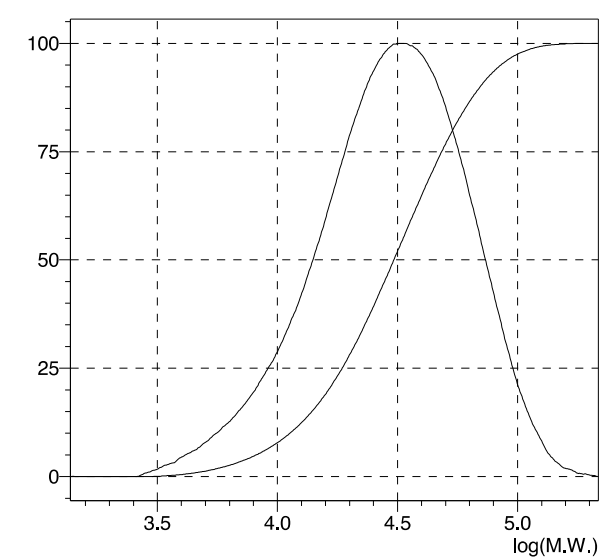

\section{GPC Calculation Results}

Peak\#:1 (Detector A Ch1)

[Peak Information]

\begin{tabular}{lrrrr} 
& Time $(\mathrm{min})$ & Volume $(\mathrm{mL})$ & Molecular Weight & \multicolumn{1}{c}{ Height } \\
Start & 12.492 & 12.492 & 217533 & 212 \\
Top & 14.392 & 14.392 & 30539 & 623 \\
End & 17.400 & 17.400 & 1365 & 380
\end{tabular}

Area : 64347

Area\%: 100.0000

[Average Molecular Weight]

Number Average Molecular Weight(Mn) 22286

Weight Average Molecular Weight(Mw) 36708

Z Average Molecular Weight(Mz) 53597

Z+1 Average Molecular Weight(Mz1) $\quad 71266$

$\mathrm{Mw} / \mathrm{Mn} \quad 1.64718$

$\mathrm{Mv} / \mathrm{Mn} \quad 1.55343$

$\mathrm{Mz} / \mathrm{Mw} \quad 1.46008$

Detector A Ch1

[Average Molecular Weight(Total)]

Number Average Molecula

Weight Average Molecular Weight(Mw) 36708

$\mathrm{Z}$ Average Molecular Weight(Mz) 53597

Z+1 Average Molecular Weight(Mz1) 71266

$\mathrm{Mw} / \mathrm{Mn} \quad 1.64718$

$\mathrm{Mv} / \mathrm{Mn} \quad 1.55343$

$\mathrm{Mz} / \mathrm{Mw} \quad 1.46008$

Figure 105. SEC traces of isolated PLA prepared via ROP of rac-lactide initiated by the $\mathbf{4 a} /{ }^{\mathrm{PrOH}} \mathrm{system}$. Conditions: 100 equiv. of rac-lactide (vs. 4a), 1 equiv. of ${ }^{i} \mathrm{PrOH},[\mathrm{rac} \text {-lactide }]_{0}=1 \mathrm{M}$, dichloromethane, room temperature, $2 \mathrm{~h}$ : $98 \%$ conversion to PLA. 


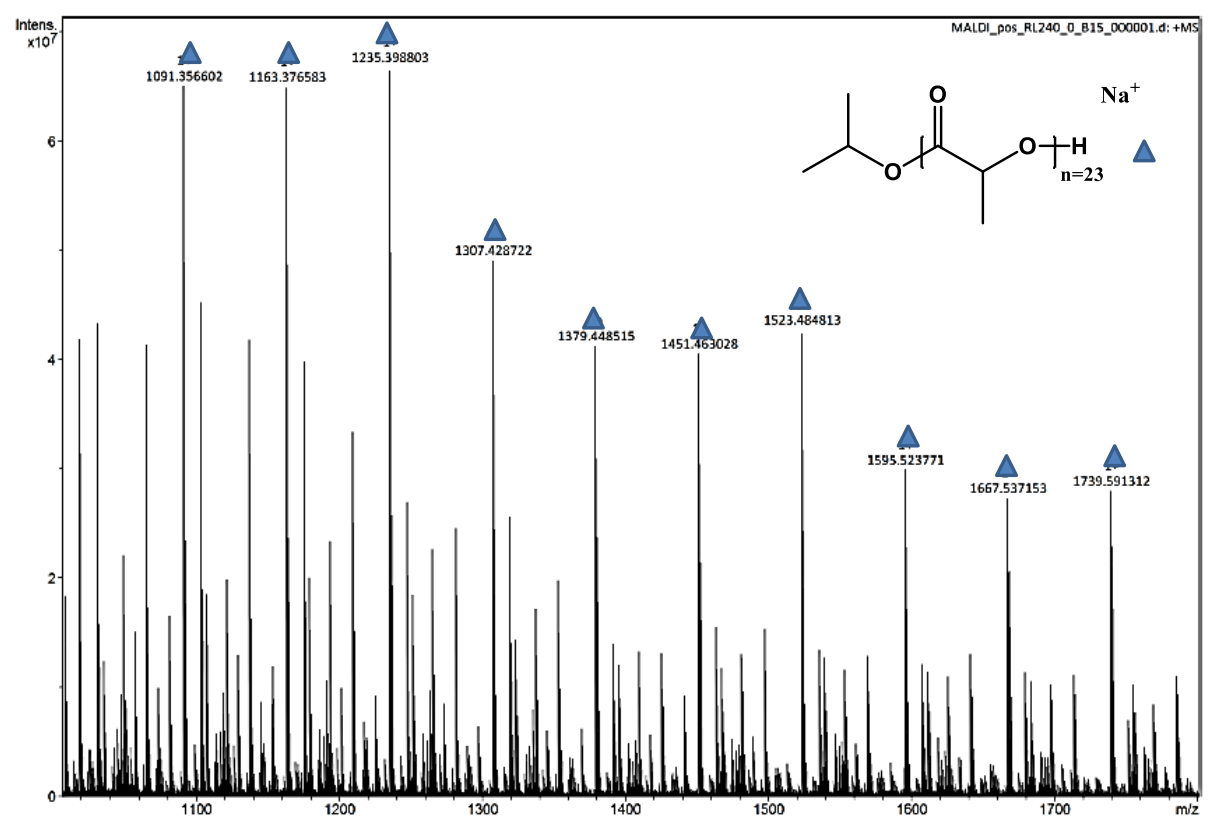

Figure S106. MALDI-TOF spectra of isolated PLA prepared via ROP of rac-lactide initiated by the $4 \mathbf{a} /{ }^{i} \mathrm{PrOH}$ system. Conditions: 100 equiv. of $r a c$-lactide (vs. 4a), 1 equiv. of ${ }^{i} \mathrm{PrOH}(v s . \mathbf{4 a}),[\mathrm{rac} \text {-lactide }]_{0}=1 \mathrm{M}, \mathrm{CH}_{2} \mathrm{Cl}_{2}, \mathrm{RT}, 2 \mathrm{~h}$.

\section{Catalyst 1b}

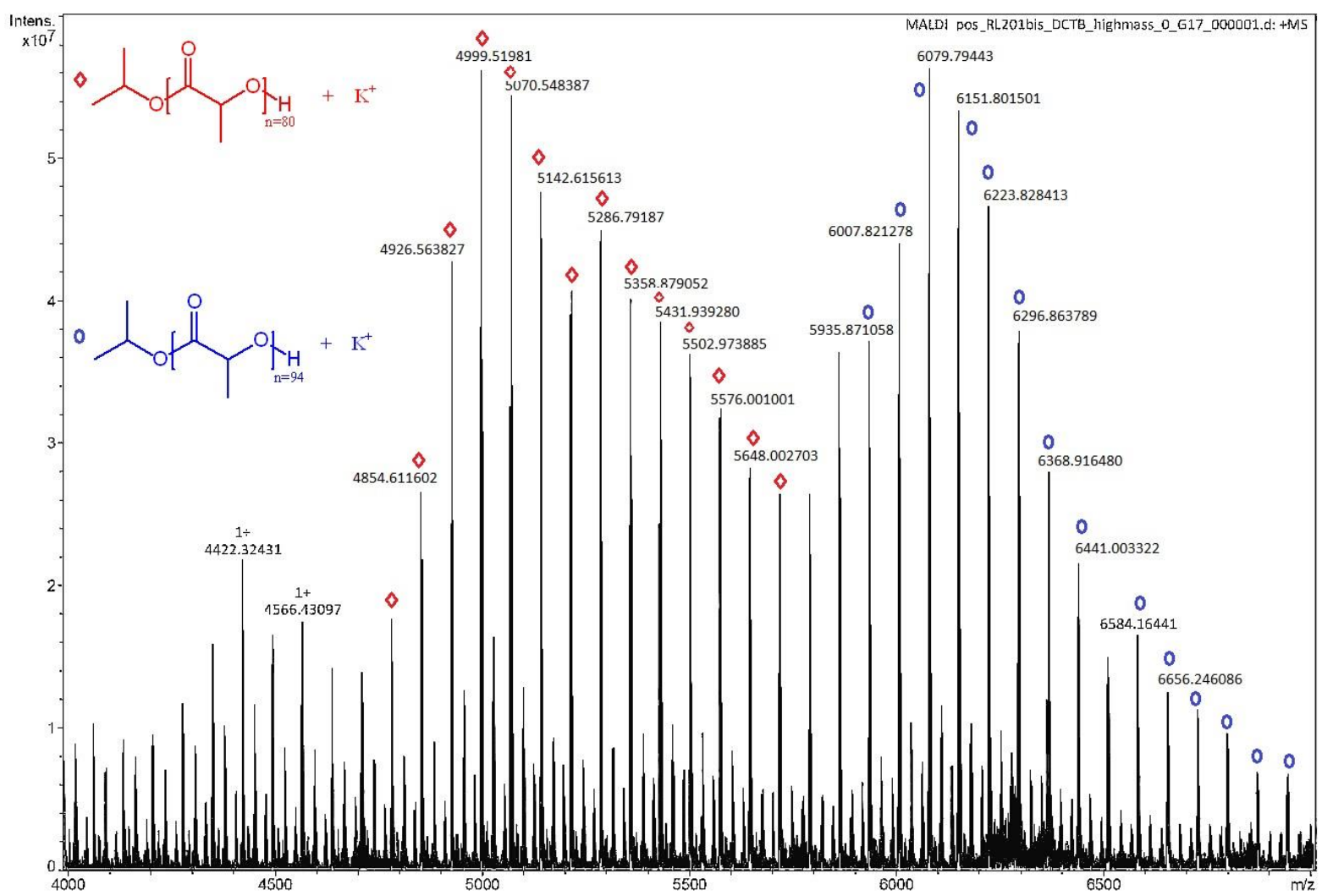

Figure S107. MALDI-TOF spectra of isolated PLA prepared via ROP of rac-lactide initiated by the $1 \mathbf{b} /{ }^{i} \mathrm{PrOH}$ system. Conditions: 100 equiv. of $r a c$-lactide (vs. 1b), 1 equiv. of ${ }^{i} \mathrm{PrOH}(v s . \mathbf{1 b}),[\text { rac-lactide }]_{0}=1 \mathrm{M}$, toluene, $70{ }^{\circ} \mathrm{C}, 24 \mathrm{~h}$. 


\section{Catalyst 2b}

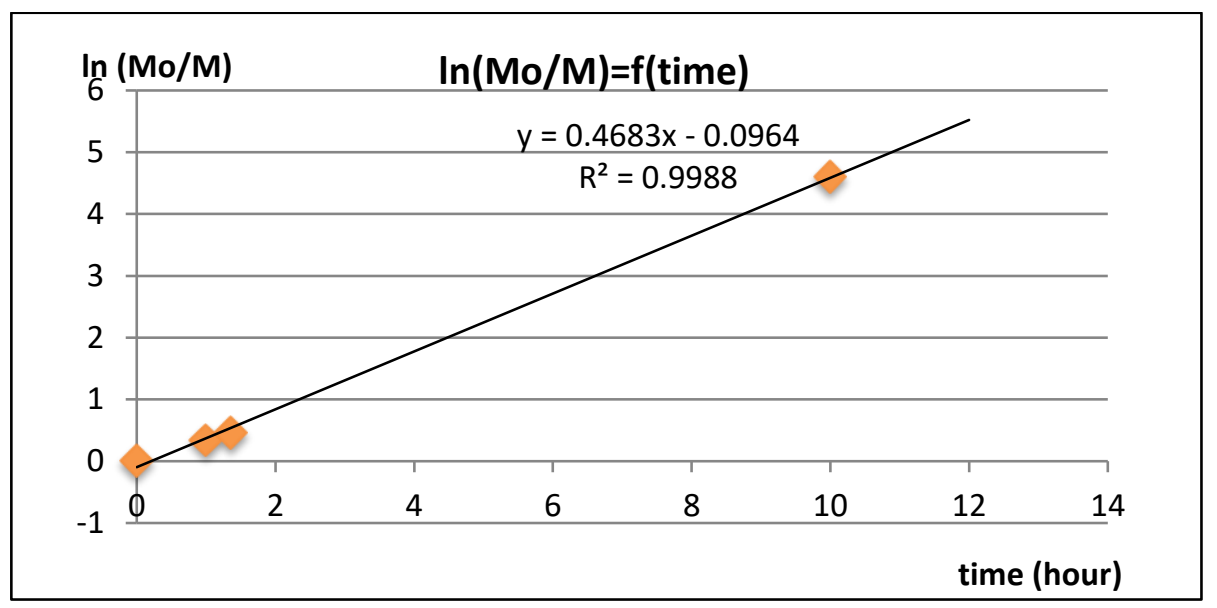

Figure S108. Plot of $\ln (\mathrm{Mo} / \mathrm{M})$ as a function of the time in the ROP of $r a c$-lactide using the complex $\mathbf{2 b} / \mathrm{BnOH}$ system. Conditions: 100 equiv. of $r a c$-lactide ( $v$ s. 2b), 1 equiv of $\mathrm{BnOH},[\mathrm{rac} \text {-lactide }]_{0}=1 \mathrm{M}$, toluene, $90{ }^{\circ} \mathrm{C}, 10 \mathrm{~h} .100 \%$ conversion to PLA.

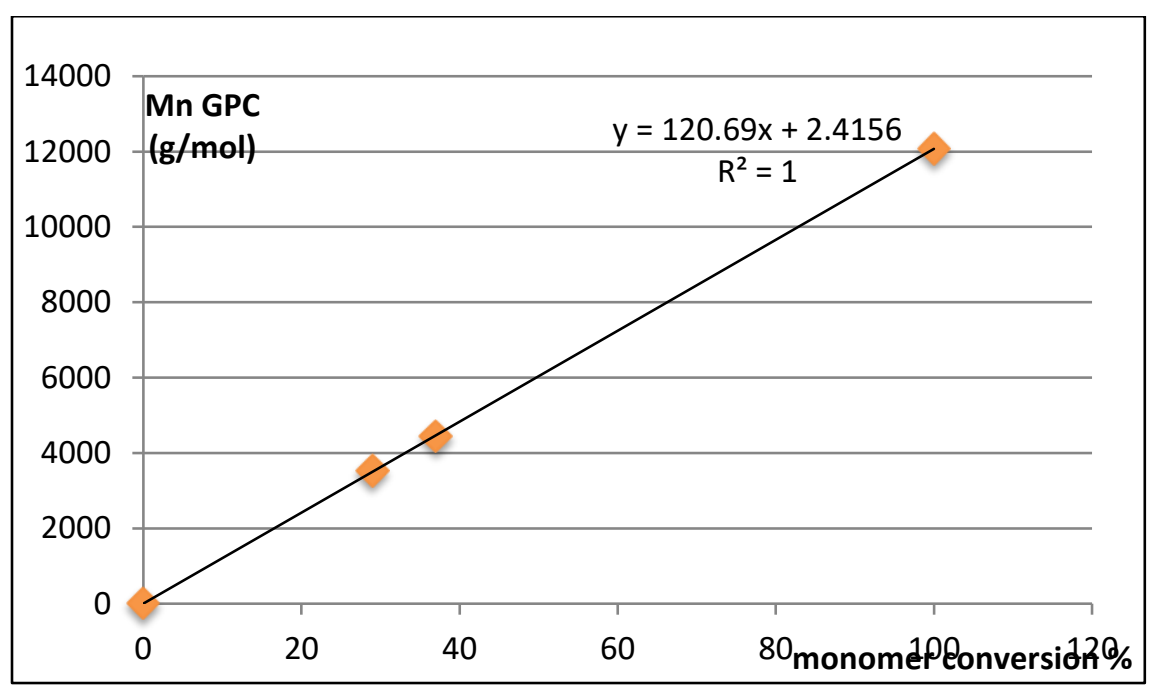

Figure S109. Graph of the PLA $M_{\mathrm{n}}$ values as a function of lactide conversion for the ROP of $r a c$-lactide initiated by the $\mathbf{2 b}$ / $\mathrm{BnOH}$ system. Conditions: 100 equiv. of rac-lactide (vs. 2b), 1 equiv. of $\mathrm{BnOH}(v s . \mathbf{2 b}),[\mathrm{M}]_{0}=[\mathrm{rac} \text {-lactide }]_{0}=1 \mathrm{M}$, toluene, $90{ }^{\circ} \mathrm{C}, 10 \mathrm{~h}, 100 \%$ conversion to PLA. 
$====$ Shimadzu LCsolution GPC Analysis Report

$\begin{array}{ll}\text { Acquired by } & : \text { Admin } \\ \text { Sample Name } & : \text { ab331fin } \\ \text { Sample ID } & : \text { ab331fin } \\ \text { Vail\# } & : \\ \text { Injection Volume } & : 100 \mathrm{uL} \\ \text { Data Filename } & : \text { ab331fin.lcd } \\ \text { Method Filename } & : \text { gpc.lcm } \\ \text { Batch Filename } & : \\ \text { Report Filename } & : \text { Report_template.lcr } \\ \text { Date Acquired } & : 05 / 06 / 2018 \text { 11:36:03 } \\ \text { Data Processed } & : 05 / 06 / 2018 \text { 12:06:05 }\end{array}$

Chromatogram \& Calibration Curve

$\mathrm{mV}$

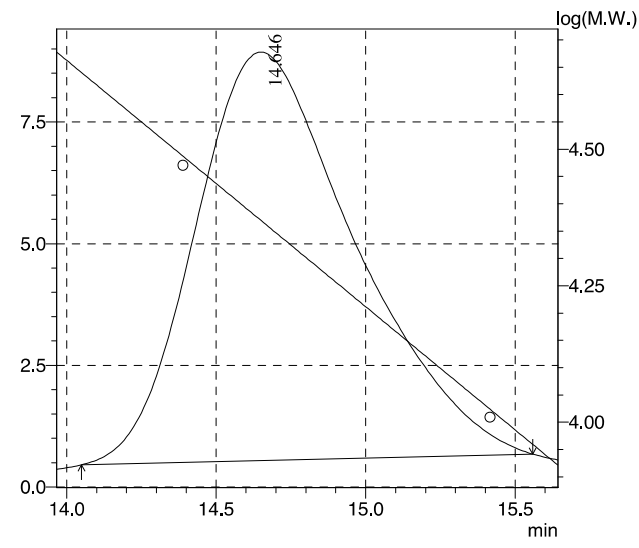

Molecular Weight Distribution Curve

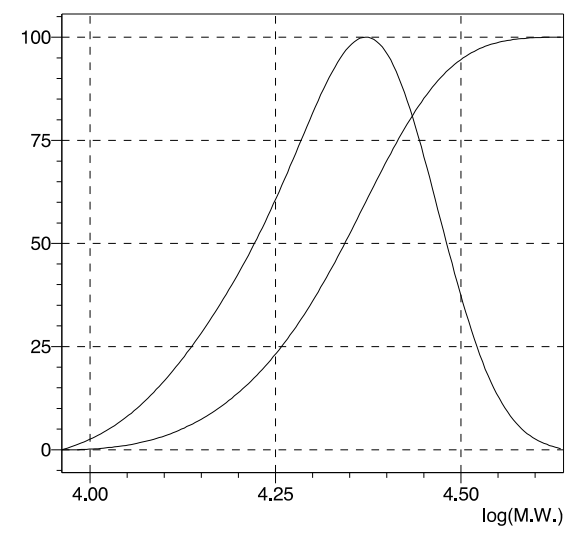

\section{GPC Calculation Results}

Peak\#: 1 (Detector A Ch1)

[Peak Information]

\begin{tabular}{lcrrr} 
& Time $(\min )$ & Volume $(\mathrm{mL})$ & Molecular Weight & \multicolumn{1}{c}{ Height } \\
Start & 14.050 & 14.050 & 43662 & 456 \\
Top & 14.646 & 14.646 & 23519 & 8391 \\
End & 15.558 & 15.558 & 9114 & 678
\end{tabular}

Area : 311828

Area\%: 100.0000

[Average Molecular Weight]

Number Average Molecular Weight(Mn) 20816

Weight Average Molecular Weight(Mw) $\quad 22314$

Z Average Molecular Weight(Mz) 23749

Z+1 Average Molecular Weight(Mz1) 25097

$\begin{array}{ll}\mathrm{Mw} / \mathrm{Mn} & 1.07199\end{array}$

$\begin{array}{ll}\mathrm{Mw} / \mathrm{Mn} & 1.07199 \\ \mathrm{Mv} / \mathrm{Mn} & 1.06239\end{array}$

$\begin{array}{ll}\mathrm{Mz} / \mathrm{Mw} & 1.06429\end{array}$

Detector A Ch1

[Average Molecular Weight(Total)]

Number Average Molecular Weight(Mn) 20816

$\begin{array}{ll}\text { Number Average Molecular Weight(Mn) } & 20816 \\ \text { Weight Average Molecular Weight(Mw) } & 22314\end{array}$

Z Average Molecular Weight(Mz) 23749

Z+1 Average Molecular Weight(Mz1) $\quad 25097$

$\mathrm{Mw} / \mathrm{Mn} \quad 1.07199$

$\mathrm{Mv} / \mathrm{Mn} \quad 1.06239$

$\mathrm{Mz} / \mathrm{Mw}$

Figure S110. SEC traces of isolated PLA prepared via ROP of rac-lactide initiated by the 2b/BnOH system. Conditions: 100 equiv. of rac-lactide (vs. 2b), 1 equiv. of $\mathrm{BnOH},[\mathrm{rac} \text {-lactide }]_{0}=1 \mathrm{M}$, toluene, $90{ }^{\circ} \mathrm{C}, 10 \mathrm{~h}: 100 \%$ conversion to PLA. 


\section{===" Shimadzu LCsolution GPC Analysis Report ===}

$\begin{array}{ll}\text { Acquired by } & : \text { Admin } \\ \text { Sample Name } & : \text { ab366f1 } \\ \text { Sample ID } & : \text { ab366f1 } \\ \text { Vail\# } & : \\ \text { Injection Volume } & : 100 \mathrm{uL} \\ \text { Data Filename } & : \text { ab366f1.lcd } \\ \text { Method Filename } & : \text { gpc.lcm } \\ \text { Batch Filename } & : \\ \text { Report Filename } & : \text { Report_template.lcr } \\ \text { Date Acquired } & : 14 / 11 / 201810: 19: 15 \\ \text { Data Processed } & : 14 / 11 / 201810: 49: 17\end{array}$

\section{Chromatogram \& Calibration Curve}

$\mathrm{mV}$

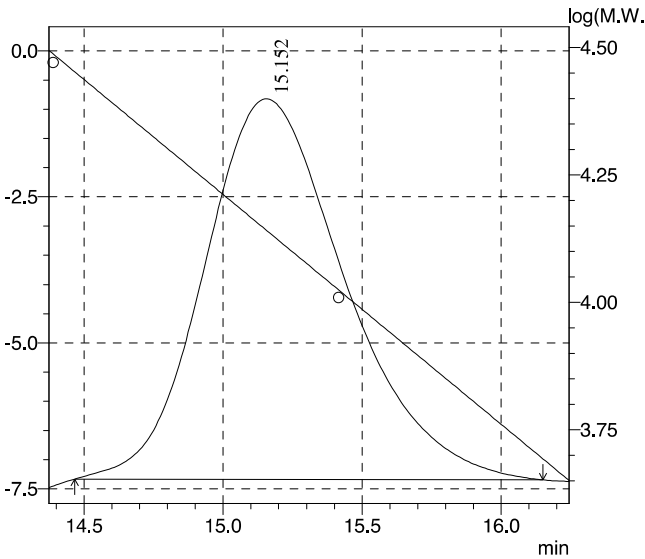

Molecular Weight Distribution Curve

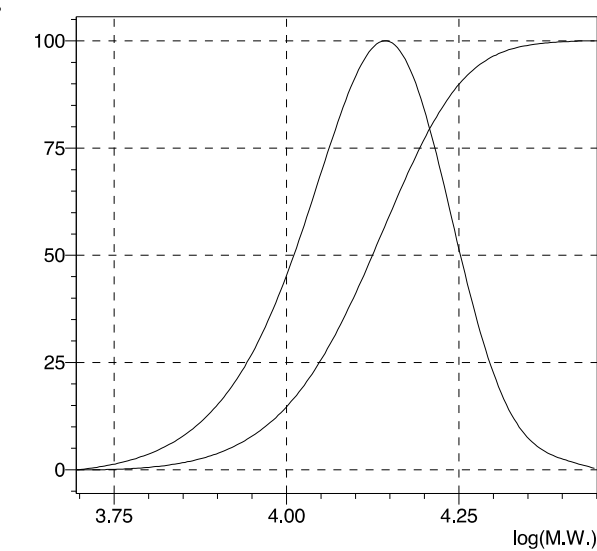

GPC Calculation Results

Peak\#:1 (Detector A Ch1)

[Peak Information]

\begin{tabular}{lrrrr} 
& Time $(\mathrm{min})$ & Volume $(\mathrm{mL})$ & Molecular Weight & \multicolumn{1}{c}{ Height } \\
Start & 14.467 & 14.467 & 28323 & -7330 \\
Top & 15.152 & 15.152 & 13905 & 6512 \\
End & 16.150 & 16.150 & 4929 & -7345
\end{tabular}

Area : 229437

Area\% : 100.0000

[Average Molecular Weight]

Number Average Molecular Weight(Mn) $\quad 12689$

Weight Average Molecular Weight(Mw) 13551

Z Average Molecular Weight(Mz)

Z+1 Average Molecular Weight(Mz1) $\quad 15210$

Mw/Mn $\quad 1.06798$

$\begin{array}{ll}\mathrm{Mv} / \mathrm{Mn} & 1.05887\end{array}$

$\mathrm{Mz} / \mathrm{Mw} \quad 1.06192$

Detector A Ch1

[Average Molecular Weight(Total)]

Number Average Molecular Weight(Mn) $\quad 12689$

Weight Average Molecular Weight(Mw) 13551

$Z$ Average Molecular Weight(Mz) 14390

Z+1 Average Molecular Weight(Mz1) $\quad 15210$

$\mathrm{Mw} / \mathrm{Mn} \quad 1.06798$

$\mathrm{Mv} / \mathrm{Mn} \quad 1.05887$

$\mathrm{Mz} / \mathrm{Mw} \quad 1.06192$

Figure S111. SEC traces of isolated PLA prepared via ROP of $r a c$-lactide initiated by the $2 \mathbf{b} /{ }^{i} \mathrm{PrOH}$ system. Conditions: 100 equiv. of rac-lactide (vs. 2b), 1 equiv. of ${ }^{i} \mathrm{PrOH},[\mathrm{rac} \text {-lactide }]_{0}=1 \mathrm{M}$, toluene, $70{ }^{\circ} \mathrm{C}, 24 \mathrm{~h}$ : $56 \%$ conversion to PLA. 


\section{==== Shimadzu LCsolution GPC Analysis Report}

$\begin{array}{ll}\text { Acquired by } & : \text { Admin } \\ \text { Sample Name } & : \text { ab367f1 } \\ \text { Sample ID } & : \text { ab367f1 } \\ \text { Vail\# } & : \\ \text { Injection Volume } & : 100 \mathrm{uL} \\ \text { Data Filename } & : \text { ab367f1.lcd } \\ \text { Method Filename } & : \text { gpc.lcm } \\ \text { Batch Filename } & : \\ \text { Report Filename } & : \text { Report_template.lcr } \\ \text { Date Acquired } & : 14 / 11 / 201811: 12: 57 \\ \text { Data Processed } & : 14 / 11 / 201811: 43: 01\end{array}$

\section{Chromatogram \& Calibration Curve}

$\mathrm{mV}$

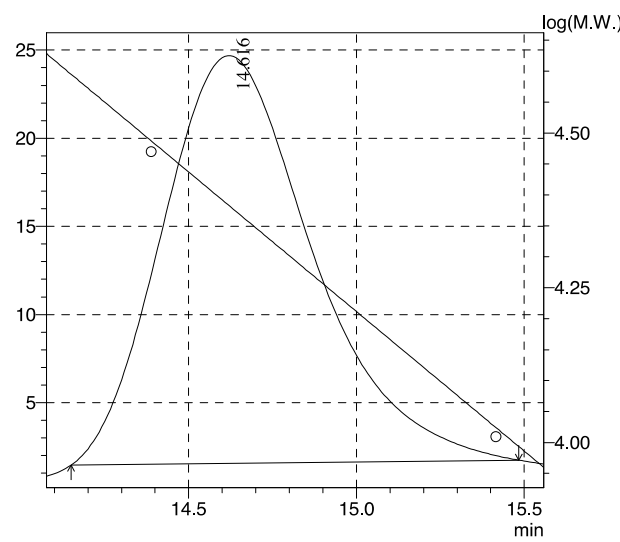

Molecular Weight Distribution Curve

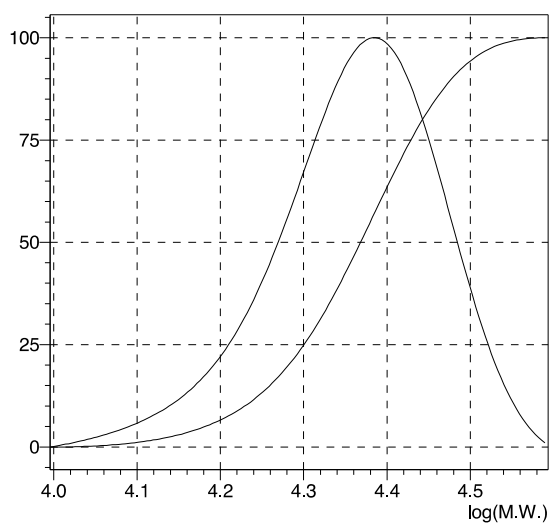

\section{GPC Calculation Results}

\begin{tabular}{|c|c|c|c|c|}
\hline & Time(min) & Volume(mL) & Molecular Weight & Height \\
\hline Start & 14.150 & 14.150 & 39354 & 1453 \\
\hline Top & 14.616 & 14.616 & 24243 & 23136 \\
\hline End & 15.483 & 15.483 & 9852 & 1734 \\
\hline
\end{tabular}

Area : 718792

Area\%: 100.0000

[Average Molecular Weight]

Number Average Molecular Weight(Mn) 22385

Weight Average Molecular Weight(Mw) 23540

Z Average Molecular Weight(Mz) 24620

Z+1 Average Molecular Weight(Mz1) 25626

Mw/Mn $\quad 1.05159$

$\mathrm{Mv} / \mathrm{Mn}$

$\mathrm{Mz} / \mathrm{Mw}$

1.04480

Detector A Ch1

[Average Molecular Weight(Total)]

Number Average Molecular Weight(Mn)

Weight Average Molecular Weight(Mw)

Z Average Molecular Weight(Mz)

$\mathrm{Z}+1$ Average Molecular Weight(Mz1)

$\mathrm{Mw} / \mathrm{Mn}$

$\mathrm{Mv} / \mathrm{Mn}$

1.04588

$\mathrm{Mz} / \mathrm{Mw}$

Figure S112. SEC traces of isolated PLA prepared via ROP of $r a c$-lactide initiated by the $2 \mathbf{b} /{ }^{i} \mathrm{PrOH}$ system. Conditions: 100 equiv. of $r a c$-lactide ( $v s .2 \mathbf{2 b}$ ), 1 equiv. of ${ }^{i} \mathrm{PrOH},[\text { rac-lactide }]_{0}=1 \mathrm{M}$, toluene, $90{ }^{\circ} \mathrm{C}, 12 \mathrm{~h}: 100 \%$ conversion to PLA. 


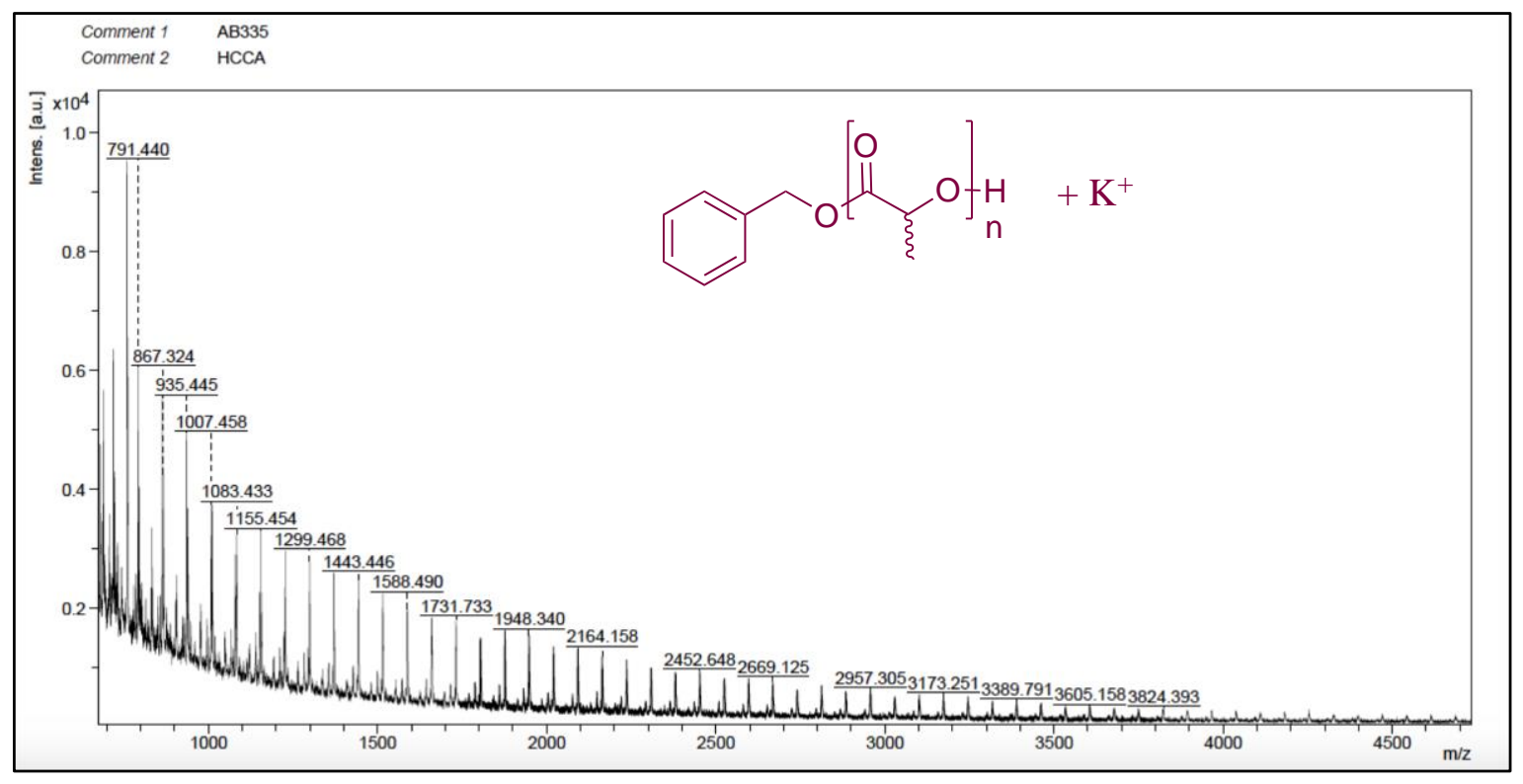

Figure S113. MALDI-TOF spectra of an isolated PLA oligomer prepared via ROP of rac-lactide initiated by the $\mathbf{2 b} / \mathrm{BnOH}$ system. (Conditions: 20 equiv. of $r a c$-lactide (vs. 2b), 1 equiv. of $\mathrm{BnOH}(v s . \mathbf{2 b}),[\text { rac-lactide }]_{0}=1 \mathrm{M}$, toluene, $90{ }^{\circ} \mathrm{C}, 10 \mathrm{~h}$ ).

\section{Catalyst 3b}

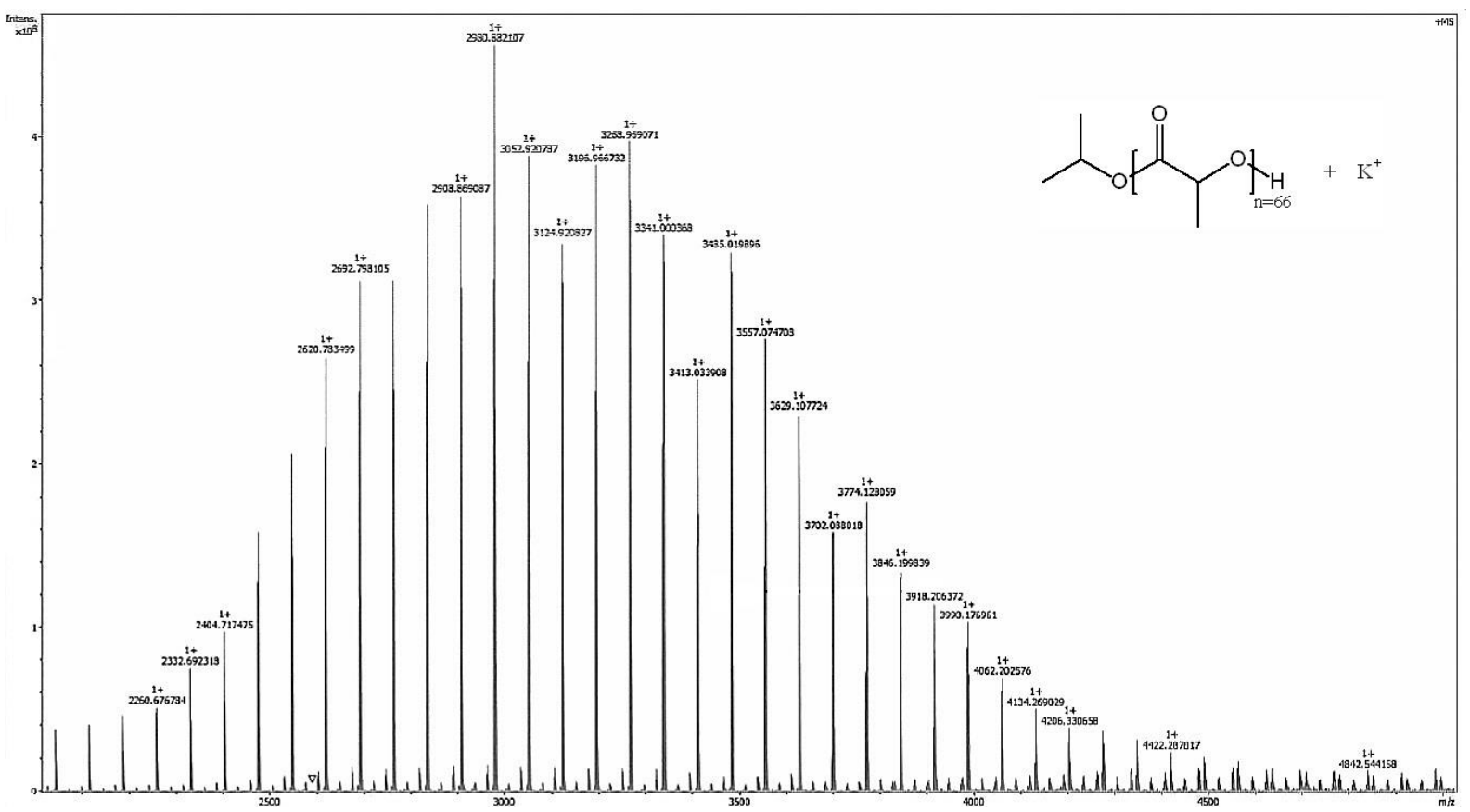

Figure S114. MALDI-TOF spectra of an isolated PLA oligomer prepared via ROP of rac-lactide initiated by the $3 \mathbf{b} / \mathrm{BnOH}$ system. (Conditions: 20 equiv. of rac-lactide (vs. 3b), 1 equiv. of $\mathrm{BnOH}(v s .3 \mathbf{b}),[\text { rac-lactide }]_{0}=1 \mathrm{M}$, toluene, $70{ }^{\circ} \mathrm{C}, 24 \mathrm{~h}$ ). 